\title{
GIS-gestützte \\ Regionalisierung von Klima- und Depositionsdaten in Niedersachsen
}

\author{
Dissertation
}

zur Erlangung des Doktorgrades des der Fakultät für Forstwissenschaften und Waldökologie der Georg-August-Universität Göttingen

\author{
vorgelegt von \\ Volker Mues \\ geb. in Frankfurt/Main
}

Göttingen 2000 
D7

1. Berichterstatter: Prof. Dr. Dr. h.c. B. Sloboda

2. Berichterstatter: Prof. Dr. G. Gravenhorst

3. Berichterstatter: Prof. Dr. J. Saborowski

Tag der mündlichen Prüfung: 13. April. 2000

Veröffentlicht unter: http://webdoc.sub.gwdg.de/diss/2000/mues/ 


\section{Danksagung}

Herrn Prof. Dr. Dr. h.c. B. SLOBODA möchte ich für die Themenstellung und die ausgezeichneten Arbeitsbedingungen in seinem Institut für Forstliche Biometrie und Informatik danken. Ohne die von ihm geschaffenen Voraussetzungen wären die Durchführung und das Gelingen der Dissertation nicht möglich gewesen. Bei auftretenden Problemen jeder Art konnte ich mich stets auf seine Unterstützung verlassen.

Dr. MARTIN JANSEN bin ich sehr dankbar für viele Literaturhinweise und die kritische Beobachtung meiner Arbeit, die mir immer wieder inhaltliche Orientierung gab. Für die Einführung in die Geostatistik, sowie für viele hilfreiche Ideen und Anregungen danke ich Prof. Dr. J. SABOROWSKI, dessen logisches und analytisches Denken zur Lösung vieler Probleme führte. Die Einweisung im Bereich des Geographischen Informationssystems ARC/Info durch Dipl. Geogr. RAINER SCHULZ und die konstruktive Zusammenarbeit mit ihm ermöglichten mir eine sehr vielseitige Arbeitsweise.

Für das Bereinigen der unausweichlichen „Katastrophen“ im Bereich der EDV danke ich Dr. ReINHOlD MeYer, der immer einen Ausweg kennt. Auch die Damen aus dem Institutssekretariat, Frau Watteler und Frau Bornemann, wußten stets Rat.

Ein besonderer Dank gilt Jaroslav Myşiak, der für ein hervorragendes Arbeitsklima im GIS-Raum sorgte, aber auch allen anderen Mitstreitern.

Nicht zuletzt gilt mein Dank meiner Familie, insbesondere meiner Frau Sabine für die mir geschaffenen Freiräume. 


\section{Inhaltsverzeichnis}

Deckblatt und Berichterstatter

Danksagung

Inhaltsverzeichnis

1 EINLEITUNG

1.1 Ziel der Arbeit

2 REGIONALISIERUNGSMETHODIK

2.1 Mittelwertbildung

2.2 Numerische Interpolation

2.3 Geostatistik

2.4 Berücksichtigung externer Effekte

2.5 Beschreibung der angewandten Methodik

\section{RÄUMLICHE VARIABLEN ZUR BESCHREIBUNG DER MEBSITUATION}

3.1 Fehler durch ungenaue Verortung der Meßstationen

3.2 Lageabhängige Variablen

3.2.1 Koordinaten

3.2.2 Abstand zur Küste

3.3 Höhenabhängige Variablen

3.3.1 Geländehöhe

3.3.2 Hangneigung und Hangrichtung

3.3.3 Lee-Indices

3.3.4 Luv-Index

3.3.5 Relative Exponiertheit

3.3.6 Kaltluftabfluß

3.3.7 Insolation

3.4 Variablen aus Kartierungen 


\subsubsection{Landnutzungsklassen}

\subsubsection{Fachdaten}

\subsubsection{Forsteinrichtung \\ 3.4.2.2 Interzeptionsindex}

\section{VERWENDETE DATEN}

\subsection{Datenbasis Klima}

4.1.1 Verortung der Stationen und Berücksichtigung von Meßortverlegungen

\subsubsection{Lufttemperatur}
4.1.2.1 Lufttemperatur im Bergland
4.1.2.2 Lufttemperatur im Tiefland

\subsubsection{Niederschlagssumme}
4.1.3.1 Niederschlagssumme im Bergland
4.1.3.2 Niederschlagssumme im Tiefland

\subsubsection{Relative Luftfeuchte}
4.1.4.1 Relative Luftfeuchte im Bergland
4.1.4.2 Relative Luftfeuchte im Tiefland

\subsection{Depositionsdaten}

\subsubsection{Prozesse der Deposition}

\subsubsection{Verwendete Datenkollektive}

\subsubsection{Zielvariablen der Depositionsregionalisierung}

\subsubsection{Repräsentanz der Depostionsdatenkollektive}

\subsubsection{Besonderheit der Regionalisierung der Depositionen}

\section{ERGEBNISSE}

\section{$5.1 \quad$ Klimaelemente}

\subsubsection{Lufttemperatur}

5.1.1.1 Lufttemperatur im Bergland

5.1.1.2 Lufttemperatur im Tiefland

\subsubsection{Niederschlagssumme}

5.1.2.1 Niederschlagssumme im Bergland

5.1.2.2 Niederschlagssumme im Tiefland

\subsubsection{Relative Luftfeuchte}

5.1.3.1 Relative Luftfeuchte im Bergland

5.1.3.2 Relative Luftfeuchte im Tiefland 


\subsection{Depositionen}

5.2.1 Ausschließliche Betrachtung der Freilanddepositionen

5.2.2 Integration der Bestandesdepositionen

\section{DISKUSSION}

\subsection{Klimaelemente}

6.1.1 Lufttemperatur

6.1.1.1 Lufttemperatur im Bergland

6.1.1.2 Lufttemperatur im Tiefland

6.1.2 Niederschlagssumme

6.1.2.1 Niederschlag im Bergland

6.1.2.2 Niederschlag im Tiefland

6.1.3 Relative Luftfeuchte

6.1.3.1 Relative Luftfeuchte im Bergland

6.1.3.2 Relative Luftfeuchte im Tiefland

\subsection{Depositionen}

6.2.1 Ausschließliche Betrachtung der Freilanddepositionen

6.2.2 Integration der Bestandesdepositionen

\section{ZUSAMMENFASSUNG}

\subsection{Klimaelemente}

7.2 Depositionen

8 LITERATURLISTE

9 ANHANG

10 GRAPHISCHE DARSTELLUNG DER ERGEBNISSE

Ergebnisdarstellung 1-4

Ergebnisdarstellung 5-8

Ergebnisdarstellung 9-12

Ergebnisdarstellung 13-16

Ergebnisdarstellung 17-20

Ergebnisdarstellung 21-22 
Lebenslauf

Eidesstattliche Versicherung

Dissertation zum Download 


\section{Einleitung}

Raumrelevante Entscheidungen werden zumeist in Form von „Plänen“ oder „Programmen“ festgelegt (Landschaftsrahmenpläne, Bebauungspläne oder Waldbauprogramme, Entwicklungsprogramme, usf.). Diese dienen der Darstellung von Ergebnissen oft sehr komplexer Prozesse der Entscheidungsfindung. Die Komplexität ist zumeist bedingt durch die Vielzahl von Personen, Personengruppen, Interessenverbände, Verwaltungen, etc., deren Belange von der zu fällenden Entscheidung betroffen sind. Alle Betroffenen haben dabei oft stark voneinander abweichende Zielvorstellungen, die es bei der Entscheidungsfindung zu berücksichtigen gilt. Die Akzeptanz der Entscheidung durch die Betroffenen ist jedoch nicht ausschließlich von der mehr oder weniger vollständigen Verwirklichung der eigenen Zielvorstellungen abhängig. Zumindest ebenso bedeutsam ist die Transparenz des Prozesses der Entscheidungsfindung, der sich zu diesem Zweck auf die Verknüpfung nachvollziehbarer Fakten stützen sollte. Diese Fakten sind im Falle raumrelevanter Entscheidungen zumeist Informationen über das von der Entscheidung betroffene Gebiet. Sichere räumliche Informationen sind somit die unabdingbare Basis für die Akzeptanz raumrelevanter Entscheidungen.

Selten liegen die benötigten raumbezogenen Informationen flächendeckend vor. Selbst Karten liefern zumeist keine flächig ermittelten Informationen, die an jedem Punkt des Raumes gewonnen wurden. Sie sind vielmehr eine generalisierte und durch komplexe Prozesse entwickelte Abbildung der Realität. So vermittelt beispielsweise eine Forsteinrichtungskarte zunächst den Eindruck, eine flächendeckende und flächenscharfe Abbildungen der Bestände zu sein. Die zu ihrer Herstellung verwendeten Informationen wurden jedoch durch stichprobenartige Erfassung oder Fortschreibung von zumeist ebenfalls stichprobenartig erfaßten Informationen gewonnen.

Auch die Auswertung von Luft- oder Satellitenbildern liefert keine flächenscharfe Abbildung der Realität bezüglich einer bestimmten Informationsebene. Die Darstellung der Beobachtungen in Form von Rastern - jedes „Pixel“ hat einen bestimmten Wert - hat einen stichprobenartigen und generalisierenden Effekt auf die 
Information. Dieser Effekt tritt selbst dann auf, wenn der Wert eines Pixels nicht der für den Zellmittelpunkt gemessene Wert, sondern der Mittelwert für die gesamte Rasterzelle ist. Eine Zelle entspricht der Umgebung eines Punktes, eben des Zellmittelpunktes. Jedem Punkt, der in der Umgebung des Punktes, also in der Rasterzelle liegt, wird der Wert des Rastermittelpunktes zugeordnet. Dies ist die einfachste Möglichkeit, Rasterinformation für einen diskreten Punkt zu ermitteln. Sie ist bei ausreichend genauer, d. h. feiner Rasterung allgemein als ausreichend genau anzusehen. Eine bilineare Interpolation bzw. die Berechnung von gewichteten Mittelwerten der umliegenden Rasterwerte kann alternativ für die Übertragung der Rasterinformation auf diskrete Punkte verwendet werden. Sehr grobe Raster müssen in jedem Fall zu unsicheren Werten führen, wobei man jedoch die Schrittweite eines Rasters nicht kleiner wählen sollte, um eine höhere Sicherheit und Genauigkeit vorzutäuschen, als für die Information tatsächlich gegeben ist.

Da es zwischen jedem Paar von Punkten theoretisch unendlich viele weitere Punkte gibt, ist es unmöglich, jeden Punkt in einem Gelände - zumal bei großräumiger Betrachtungsweise - bezüglich einer bestimmten Zielgröße zu beproben. Die Auswahl einer Stichprobe von punkthaften Meßorten ist daher von entscheidender Bedeutung für ein sinnvolles Verhältnis zwischen Aufwand und Nutzen bzw. Informationsgewinn. Dabei ist die Sicherheit der gewonnenen Information von entscheidender Bedeutung. Diese kann im Rahmen von Stichproben durch mehrere Faktoren beeinträchtigt werden. Neben der Meßgenauigkeit ist die Frage der Flächenrepräsentanz der Meßstellen von größter Bedeutung. FRÄNZLE (1989, S. 96ff) fordert daher z. B. die Auswahl repräsentativer Probeflächen zur Untersuchung von Ökosystemen. In vielen Fällen wird es jedoch unmöglich, oft auch unnötig sein, zur Ermittlung benötigter Information ein spezielles Meßnetz aufzubauen. Werden beispielsweise Datenreihen benötigt, die über einen längeren Beobachtungszeitraum erfaßt wurden, kann nur auf Daten bereits bestehender Meßnetze zurückgegriffen werden. Dabei ist zu berücksichtigen, daß bei der Einrichtung dieser Meßnetze nicht notwendigerweise auch das gleiche Beobachtungsziel, die gleichen Vorstellungen zur Repräsentanz des Meßnetzes Beachtung fanden, wie das für die gewünschte Informationsgewinnung angebracht $\mathrm{zu}$ sein scheint. Eine diesbezügliche Untersuchung der auszuwertenden Daten ist daher unumgänglich.

Neben bzw. nach der punkthaften (stichprobenartigen) Erfassung der gewünschten Information stellt die Übertragung der punkthaft vorliegenden Information auf die 
Fläche, die Regionalisierung, eine weitere Quelle der Unsicherheit dar. Die Regionalisierung sollte Ergebnisse liefern, die für das Zielgebiet gültig sind und möglichst genau an die Meßdaten angepaßt sind. Neben der bereits genannten Voraussetzung flächenrepräsentativer, geeigneter Meßdaten sind zwei weitere Faktoren entscheidend für eine in diesem Sinne erfolgreiche Regionalisierung: Die Regionalisierungsmethodik und das Fachwissen, das in den Regionalisierungsprozeß eingeht.

Ergebnis der Regionalisierung ist das Regionalisierungsmodell, eine Vorschrift zur Übertragung der punkthaft vorhandenen Information auf die Fläche. Es ist eine Verknüpfung von Ausgangsinformationen -i.a. Meßdaten-, und der Übertragungsmethodik unter Verwendung von Fachwissen. Die Frage stellt sich, wie diese Verknüpfung, das Regionalisierungsmodell, gestaltet werden muß, um die regionalisierte Information sicher und damit verwendbar zu machen. Dies ist sie, wie oben bereits erwähnt wurde, nur dann, wenn das Regionalisierungsmodell für das Zielgebiet gültig und möglichst genau an die Meßdaten angepaßt ist.

Die Gültigkeit eines Regionalisierungsmodells kann aus der Sicht eines bestimmten Informationsbedarfs bereits durch einen Mangel an Repräsentanz der Meßdaten eingeschränkt sein. Daher sind Repräsentanzmängel aufzudecken und die abzuleitenden Konsequenzen für die Regionalisierung und die Gültigkeit der regionalisierten Information sind zu beschreiben.

Außer durch Repräsentanzmängel der Ausgangsinformation kann die Gültigkeit eines Regionalisierungsmodells auch durch ein Übermaß an Anpassung an die Meßdaten eingeschränkt sein. Bei geringem Datenumfang werden alle zur Verfügung stehenden Datensätze zur Modellbildung und -parametrisierung benötigt. Dadurch besteht keine Möglichkeit zur Validierung. Die Genauigkeit eines Modells kann dann nur über eine Quantifizierung der Anpassung an die zugrunde liegenden Daten beschrieben werden, über seine Präzision. Mit zunehmender Anpassung an ein bestimmtes Datenkollektiv wird die Gültigkeit des entwickelten Modells für andere Datenkollektive oder für die Grundgesamtheit jedoch eingeschränkt bzw. unwahrscheinlicher. Es ist daher zwingend notwendig, neben der Anpassung an die Daten, der Präzision, auch das Kriterium der Einfachheit zur Beurteilung eines Regionalisierungsmodells heranzuziehen. 
Das dritte Kriterium zur Beurteilung von Regionalisierungsmodellen oder Modellen überhaupt ist die Plausibilität. Eine Regionalisierung überträgt punkthaft vorliegende Information auf die Fläche. Dazu muß geklärt werden, warum an den zugrunde liegenden Meßstationen gerade die vorliegenden Meßergebnisse zustande kamen und wie man die räumliche Verteilung der Meßdaten beschreiben kann.

Oft sind Daten von Meßstationen, die nah beieinander liegen, ähnlicher als solche von Meßstationen mit großer räumlicher Distanz. Diese Eigenschaft vieler Größen wird im täglichen Leben auf empirischem Wege oft unbewußt verwendet. Beispielsweise orientiert man sich bei der Wahl der Bekleidung an Lufttemperatur und Niederschlagsbeobachtungen in der Nähe des Ortes, an dem man sich tagsüber aufhalten wird. Diese räumliche Eigenschaft von Meßdaten, ihre räumliche Autokorrelation, wird im Rahmen von geostatistischen Untersuchungen beschrieben und zur Regionalisierung genutzt. Ein inzwischen häufig verwendetes Verfahren hierzu ist das KRIGING. Die zur Anwendung dieses Verfahrens benötigte Hard- und Software ist immer leichter und günstiger zugänglich, was die zunehmende Verwendung dieser zunächst für den Bergbau entwickelten Methodik im Rahmen der Regionalisierung ermöglicht.

Neben der geostatistischen Erklärung der räumlichen Verteilung von Meßdaten durch Beschreibung und Verwendung der räumlichen Autokorrelation kann eine wirkungsbezogene Erklärung $\mathrm{zu}$ einer erfolgreichen Regionalisierung der Zielvariablen beitragen. Die Realisierung der Meßwerte soll durch empirische Beziehungen zu anderen Größen bzw. Variablen vorhergesagt werden, wobei „Vorhersage“ im statistischen, nicht im zeitlichen Sinne gemeint ist. Die räumliche Verteilung der Zielvariablen wird zu diesem Zweck auf die räumliche Verteilung anderer Variablen („Prädiktoren“ oder „Regressoren“) zurückgeführt. Besteht zwischen den Variablen ausschließlich ein zufälliger Zusammenhang, der nur an den Meßorten gefunden wird, so ist keine Gültigkeit für das gesamte Untersuchungsgebiet gegeben. Nur solche für ein Datenkollektiv gefundenen, im Sinne der Präzision (Bestimmtheitsmaß) bedeutenden und nominell signifikanten Zusammenhänge sollten daher zur Regionalisierung verwendet werden, die vor dem Hintergrund des vorhandenen Fachwissens plausibel erscheinen (vgl. FRÄNZLE, 1989, S. 66f). Die Überprüfung auf Plausibilität ist aber nur dann möglich, wenn die Prädiktoren interpretierbare physikalische Größen darstellen, die nach derzeitigem Fachwissen in möglichst direktem Zusammenhang mit der Ausprägung der 
Zielvariablen stehen. Prädiktoren müssen zur Beschreibung der Meßsituation einen bedeutenden und plausiblen Beitrag leisten.

Zur Beschreibung des Zusammenhanges zwischen einer Zielvariablen und einem oder mehreren metrischen Prädiktoren kann eine Regression nach der Methode der kleinsten Quadrate erfolgen. So wird der systematische Teil der Streuung der Zielvariablen erklärt, während das oben genannte geostatistische Verfahren Kriging den zufälligen Teil der Streuung zur Regionalisierung der Zielvariablen nutzt. Beide Verfahren können auch kombiniert verwendet werden (s. z. B. SABOROWSKI \& STOCK, 1994 oder KLEIN, 1994).

Die Definition und Ableitung raumbezogener Variablen zur Beschreibung der räumlichen Situation an den Meßstationen und im gesamten Untersuchungsgebiet ist mit einem Geographischen Informationssystem (GIS) bei geeigneter Datenbasis mit relativ geringem Aufwand möglich. Ein GIS kann zusätzlich auch in anderen Phasen der Regionalisierung sinnvoll Verwendung finden und zur Erhöhung des Vertrauens in die Regionalisierungsmodelle beitragen. Das GIS ist eine organisierte Ansammlung von Computer Hard- und Software, geographischen Daten und Fachwissen, das entworfen wurde, um jede Form von geographisch referenzierter Information zu erfassen, zu speichern, zu aktualisieren, sachgerecht zu verwenden, zu analysieren und darzustellen (ESRI, 1990). Durch diese Funktionalitäten ist das GIS von der räumlichen Darstellung der Meßdaten über die Analyse räumlicher Zusammenhänge bis hin zur Darstellung der Regionalisierungsergebnisse und Berechnung abgeleiteter Informationsebenen ein wichtiges Hilfsmittel. Möglichkeiten für den (forst-) praktischen Einsatz von geographischen Informationssystemen wurden beispielsweise von STOCK \& SLOBODA (1991) und JANSEN et al. (1994) aufgezeigt.

Ob Ergebnisse der Regionalisierung im Rahmen der Entscheidungsfindung beachtet werden, ist alleine abhängig vom Vertrauen des Entscheidenden in die Erfüllung der beiden oben genannten Kriterien Gültigkeit und Genauigkeit. Wie Meßdaten und Methodik unter welchen fachlichen Voraussetzungen zum Prozeß der Regionalisierung verwendet werden, muß für den Entscheidenden nachvollziehbar sein. Wenn die Regionalisierung und damit das zur Informationsübertragung entwickelte Regionalisierungsmodell nicht nur von hoher Präzision, sondern auch 
plausibel und möglichst einfach ist, werden regionalisierte Informationen auch als sichere Basis für räumliche Entscheidungen akzeptiert.

\subsection{Ziel der Arbeit}

Ziel der vorliegenden Arbeit ist es, für punkthaft gemessene Daten zu Klimaelementen und atmogenen Depositionen möglichst einfache und plausible Regionalisierungsmodelle mit ausreichender Präzision $\mathrm{zu}$ bilden und $\mathrm{zu}$ parametrisieren, die sich sowohl auf die Beschreibung der Meßsituation als nach Möglichkeit auch auf die räumliche Autokorrelation der Meßdaten stützen. Möglichkeiten zur Unterstützung und zur Steigerung der Transparenz und Akzeptanz des Regionalisierungsprozesses durch die Verwendung eines Geographischen Informationssystems (GIS) sollen dabei dargestellt werden. 


\section{Regionalisierungsmethodik}

In den letzten Jahren hat sich das Verständnis der „Regionalisierung“ gewandelt (BECKER, 1992). Ursprünglich wurde unter diesem Begriff die Einteilung der Landschaft nach bestimmten Eigenschaften und Parametern in möglichst homogene Untergebiete, die Regionen, verstanden. Jeder Punkt wird über die Zugehörigkeit zu einer Region definiert. Informationen über einen Landschaftsteil können über die Zugehörigkeit zu einer Region in Form ihrer Nennwerte der Unterteilungskriterien ermittelt werden. Sie können aus Karten der Regionen abgelesen werden.

Diese Form der Regionalisierung entwickelte sich hin zur Übertragung naturraumrelevanter Größen (z. B. Temperatur, Niederschlag, etc.), die punkthaft an Meßstationen erhoben worden sind, auf das gesamte Zielgebiet durch mathematische und statistische Methoden. Nach heutigem Verständnis ist es das Ziel der Regionalisierung, für jedes beliebig kleine Gebiet, bzw. für jeden Punkt, die entsprechende Information - den Wert der Zielvariablen - vorherzusagen. Hierzu werden unterschiedliche Methoden verwendet. „Vorhersage“ wird im Rahmen dieser Arbeit in statistischem, ausdrücklich nicht in zeitlichem Sinne verwendet.

\subsection{Mittelwertbildung}

Um für ein Gebiet der Größe eines Bundeslandes (Niedersachsen ca. 47500 km²) einen ersten Eindruck von den klimatischen Gegebenheiten zu bekommen, kann es durchaus sinnvoll sein, Mittelwerte und andere deskriptive Statistiken der in diesem Gebiet befindlichen Meßstationen zu berechnen (s. Tabelle 12). Dies ist auch bei einer Unterteilung von Zielgebieten durchaus üblich. Die Lage der Stationen muß nur grob für die Zuordnung zu den Untergebieten/Regionen bekannt sein.

Die Genauigkeit einer durch Mittelwertbildung praktizierten Regionalisierung steigt bei sinkender intraregionaler Variabilität der Meßwerte. Im Umkehrschluß wird eine Regionalisierung über Mittelwerte ungenauer, je heterogener die Meßergebnisse - 
und damit die naturräumlichen Gegebenheiten - in einem Zielgebiet sind. Die Beschreibung der mittleren Jahrestemperatur für das Gebiet Bremen-BremerhavenOldenburg durch einen Mittelwert dort gelegener Stationen wird allgemein eine höhere Akzeptanz erfahren als ein Mittelwert für das ungleich kleinere aber stärker reliefierte Gebiet des niedersächsischen Harzes.

Die Meßwerte der untersuchten Klimaelemente und Depositionen weisen im Untersuchungsgebiet Niedersachsen eine hohe Streuung auf (vgl. Tabelle 1). Sollen z. B. Depositionsmengen für die Optimierung von Ausgleichskalkungen von Waldgebieten geschätzt werden, so ist eine genauere Quantifizierung als durch einen großräumigen Gebietsmittelwert wünschenswert (KONITZER, mdl. Mitteilung 1998).

Tabelle 1: Arithmetische Mittelwerte, Standardabweichungen und Extrema einiger Klimaelemente und Depositionsmengen in Niedersachsen

\begin{tabular}{|c|c|c|c|c|c|c|}
\hline Elemente & Einheit & $\mathbf{n}$ & Mittelw. & Stdabw. & Min. & Max. \\
\hline mittlere jährliche & {$\left[{ }^{\circ} \mathrm{C}\right]$} & 45 & 8,3 & 1,19 & 2,9 & 9,4 \\
\hline $\begin{array}{l}\text { Lufttemperatur } \\
\text { mittlere Lufttemperatur } \\
\text { im Januar }\end{array}$ & {$\left[{ }^{\circ} \mathrm{C}\right]$} & 45 & 0,3 & 1,24 & $-4,2$ & 1,7 \\
\hline $\begin{array}{l}\text { mittlere Lufttemperatur } \\
\text { im Juli }\end{array}$ & {$\left[{ }^{\circ} \mathrm{C}\right]$} & 45 & 16,4 & 1,22 & 10,3 & 17,4 \\
\hline $\begin{array}{l}\text { durchschnittliche jährliche } \\
\text { Niederschlagssumme }\end{array}$ & {$[\mathrm{mm}]$} & 453 & 832,6 & 224,99 & 545,2 & 1767,6 \\
\hline $\begin{array}{l}\text { durchschnittliche } \\
\text { Niederschlagssumme } \\
\text { im Januar }\end{array}$ & {$[\mathrm{mm}]$} & 453 & 71,7 & 26,33 & 35,0 & 182,0 \\
\hline $\begin{array}{l}\text { durchschnittliche } \\
\text { Niederschlagssumme } \\
\text { im Juli }\end{array}$ & {$[\mathrm{mm}]$} & 453 & 78,4 & 16,21 & 54,1 & 134,0 \\
\hline $\begin{array}{l}\text { mittlere jährliche } \\
\text { relative Luftfeuchte }\end{array}$ & [\%] & 44 & 80,9 & 2,18 & 76,0 & 87,0 \\
\hline $\begin{array}{l}\text { mittlerer jährlicher Eintrag an } \\
\text { Sulfat-Schwefel }\end{array}$ & {$\left[\mathrm{kg}(\mathrm{ha} \cdot \mathrm{a})^{-1}\right]$} & 69 & 20,4 & 20,92 & 8,7 & 154,3 \\
\hline $\begin{array}{l}\text { mittlerer jährlicher Eintrag an } \\
\text { Nitrat-Stickstoff }\end{array}$ & {$\left[\mathrm{kg}(\mathrm{ha} \cdot \mathrm{a})^{-1}\right]$} & 68 & 7,2 & 4,82 & 4,4 & 38,2 \\
\hline $\begin{array}{l}\text { mittlerer jährlicher Eintrag an } \\
\text { Ammonium-Stickstoff }\end{array}$ & {$\left[\mathrm{kg}(\mathrm{ha} \cdot \mathrm{a})^{-1}\right]$} & 52 & 12,2 & 8,95 & 4,8 & 42,6 \\
\hline $\begin{array}{l}\text { mittlerer jährlicher Eintrag an } \\
\text { Chlor }\end{array}$ & {$\left[\mathrm{kg}(\mathrm{ha} \cdot \mathrm{a})^{-1}\right]$} & 69 & 28,1 & 30,78 & 6,7 & 133,5 \\
\hline $\begin{array}{l}\text { mittlerer jährlicher Eintrag an } \\
\text { Protonen }\end{array}$ & {$\left[\mathrm{kg}(\mathrm{ha} \cdot \mathrm{a})^{-1}\right]$} & 53 & 0,34 & 0,56 & 0,02 & 3,09 \\
\hline
\end{tabular}

Ein Nachteil der Verwendung arithmetischer Mittelwerte von Meßergebnissen zur Regionalisierung wird deutlich, wenn eine Situation betrachtet wird, in der keine räumliche Gleichverteilung der Stationen vorliegt. So muß eine Überrepräsentanz von Bergland-Stationen bei der Ermittlung einer Durchschnittstemperatur für ganz Niedersachsen zu einer Unterschätzung des wahren Temperaturmittels führen, da die Lufttemperatur mit zunehmender Geländehöhe abnimmt. Dieses Problem kann durch 
getrennte Mittelwerte für Berg- und Tiefland umgangen werden. Eine solche Aufteilung des Zielgebietes in Untergebiete entspricht dem traditionellen Weg der Regionalisierung. Die Meßergebnisse werden derart nach Regionen unterteilt, daß die intraregionale Varianz minimal und die interregionale Varianz maximal wird.

In heterogenen Gebieten wie dem Niedersächsischen Bergland erscheint es sinnvoll, ein dichteres Netz von Meßstationen zu unterhalten als in homogeneren Gebieten. Eine solche Meßnetzverdichtung sollte durch die gewählte Regionalisierungsmethode genutzt werden und nicht, wie es im Fall der Mittelwertbildung möglich ist, zu Problemen führen.

\subsection{Numerische Interpolation}

Die in Kap. 2.1 erläuterten Überlegungen führen zu der Frage, wie die räumliche Lage von Meßstationen berücksichtigt und zur exakteren Regionalisierung genutzt werden kann. Grundvoraussetzung für die Verwendung der Lage von Meßstationen ist die genaue Ermittlung der Meßstationskoordinaten. In Deutschland werden zu diesem Zweck zumeist Koordinaten des Gauß-Krüger-Systems verwendet. Dieses basiert auf einer transversalen Mercator-Projektion der Erdoberfläche auf eine Ebene. Dabei wird an jedem 3. Längengrad eine Projektion vorgenommen, so daß das erste System den 3. Längengrad östlicher Länge als Hauptmeridian benutzt, das zweite System den 6. Längengrad östlicher Länge und so fort. Kartographische Abbildungen Niedersachsens befinden sich ihrer geographischen Lage entsprechend im Bereich vom östlichen Rand des zweiten (Emsland) zum westlichen Rand des vierten Systems (Grenze zu Sachsen Anhalt). Um im Rahmen dieser Arbeit ein einheitliches System zu verwenden, wurden alle Koordinaten in das zentrale 3. System mit dem GIS ARC/Info transformiert (Befehl <project>, s. ESRI, 1995). 
Abbildung 1: Außengrenze Niedersachsens und Darstellung des 6., 9. und 12. Längengrades östlicher Länge

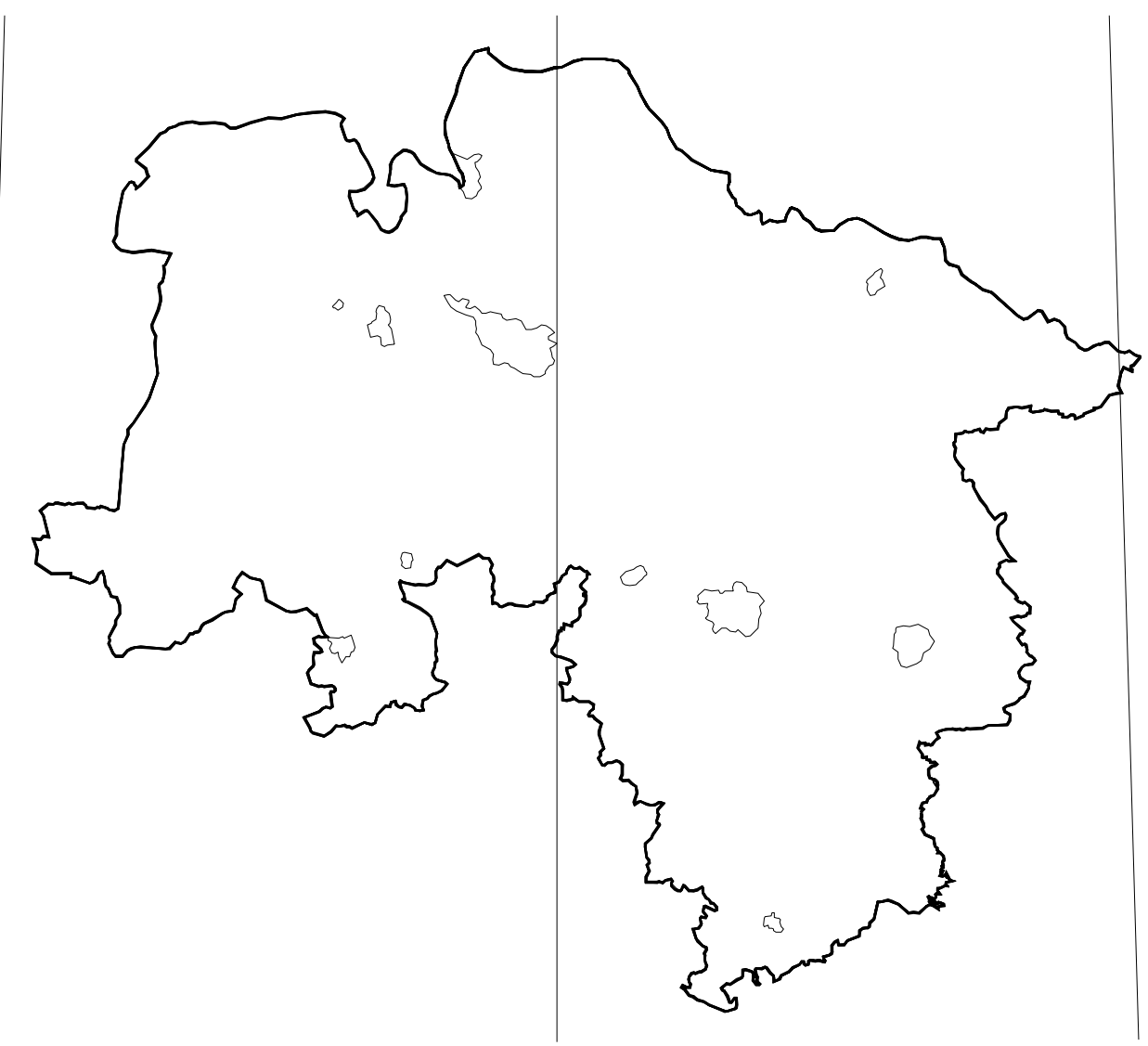

Hat man Koordinaten des Gauß-Krüger-Systems für alle Stationen zur Verfügung, so ist es möglich, beispielsweise die euklidische Entfernung zwischen zwei Meßpunkten zu berechnen. Entfernungen eines Zielpunktes zu Meßstationen können zur Interpolation der gemessenen Werte verwendet werden.

Lineare Interpolationen spielten in den Regionalisierungen von BÖHNER (1996) und BURGA \& PERRET (1998) eine Rolle. Beide Arbeiten berücksichtigen zusätzlich zu den horizontalen Koordinaten auch die Abhängigkeit der Zielvariablen von der Geländehöhe. Auf diese Beispiele wird im Rahmen von Kap. 2.4 einzugehen sein.

Eine Interpolation hat für die Regionalisierung zwei Vorteile: Erstens wird an jeder Meßstation der gemessene Wert der Zielvariable auch als Interpolationsergebnis berechnet. Die Interpolation ist in diesem Sinne eine „exakte Schätzung“ an den Meßpunkten. Angaben über die Genauigkeit einer Interpolation können näherungsweise über eine Kreuzvalidierung gewonnen werden (s. Seite 14). Zweitens wird durch Interpolation ein „unruhiger Eindruck“ vermieden, wie er beispielsweise bei einer Unterteilung des Zielgebietes in Regionen an deren Kanten entstehen kann. Eine unplausible Unstetigkeit in der Fläche wird vermieden. 
Grundgedanke der Interpolation ist die räumliche Autokorrelation. Näher gelegene Meßstationen sollen Meßwerte haben, die dem Wert am Zielpunkt ähnlicher sind als die Werte von Meßstationen mit größerer Entfernung zum Zielpunkt. Eine geläufige Interpolationsmethode ist die invers distanzgewichtete Interpolation (s. ( 1 ); vgl. ASHRAF et al., 1997 und RIPLEY, 1981, S. 36).

$$
\mathrm{z}^{*}=\sum_{\mathrm{i}=1}^{\mathrm{n}}\left(w_{\mathrm{i}} * \mathrm{x}_{\mathrm{i}}\right)
$$

Die Gewichte der Interpolation sind eine Funktion des Abstandes vom Zielpunkt mit den Koordinaten $\left(\mathrm{R}_{0}, \mathrm{H}_{0}\right)$ zu den Stützstellen mit den Koordinaten $\left(\mathrm{R}_{\mathrm{i}}, \mathrm{H}_{\mathrm{i}}\right)$ für $\mathrm{i}=1,2, \ldots, \mathrm{n}$. Ausgehend von diesem Abstand $\mathrm{d}_{0 \mathrm{i}}$ werden die Gewichte nach ( 2 ) wie folgt bestimmt:

$$
w_{\mathrm{i}}=\frac{f\left(\mathrm{~d}_{0 \mathrm{i}}\right)}{\sum f\left(\mathrm{~d}_{0 \mathrm{i}}\right)} \quad \text { mit } f\left(\mathrm{~d}_{0 \mathrm{i}}\right)=\frac{1}{\left(\mathrm{~d}_{0 \mathrm{i}}\right)^{\mathrm{b}}}
$$

Nimmt die Konstante b den Wert 1 bzw. 2 an, so handelt es sich um die einfache invers distanzgewichtete bzw. die invers quadratisch distanzgewichtete Interpolation. Die Festlegung von b ist eine subjektive Entscheidung.

Zudem kann die Auswahl der Stützstellen vom Regionalisierenden definiert werden. Dies kann entweder durch die Festlegung einer festen Anzahl von nächstgelegenen Stuitzstellen, über die Definition einer geeigneten Umgebung um den Zielpunkt oder durch eine Kombination dieser Möglichkeiten geschehen.

\subsection{Geostatistik}

Die mit der inversen Distanz oder der inversen quadrierten Distanz gewichtete Mittelung wurde von ASHRAF et al. (1997) mit der geostatistischen Interpolationsmethode Kriging verglichen, die im weiteren Sinn zu den gewichteten Mittelwertbildungen zu zählen ist. Es zeigte sich, daß Kriging und Co-Kriging hinsichtlich der Genauigkeit der Schätzungen anderen Interpolationsverfahren überlegen waren. Die u. a. von MATHERON (1963) und RIPLEY (1981) beschriebene Interpolationsmethode Kriging entzieht die Entscheidung, wie die Distanz zwischen Zielpunkten und Stützstellen gewichtet werden soll, weitestgehend subjektiven 
Einflüssen und führt zu einer Optimallösung, was den Unterschied und Vorteil anderen Interpolationsmethoden gegenüber ausmacht.

Die Gewichtung wird im Falle des Kriging durch die räumliche Autokorrelation festgelegt, welche häufig durch ein Semivariogramm beschrieben wird. Das Semivariogramm ist „das wichtigste Instrument in der Geostatistik und muß zunächst aus den Daten geschätzt werden" (BERKE \& BUSCH, 1994). Unter der Annahme der Stationarität und Isotropie - die räumliche Autokorrelation ist nur distanz- nicht aber richtungs- oder ortsabhängig - wird das Semivariogramm für beliebige Punkte $\mathrm{x}_{\mathrm{i}}, \mathrm{x}_{\mathrm{j}} \in 3_{2}$ mit $\left|\mathrm{x}_{\mathrm{i}}-\mathrm{x}_{\mathrm{j}}\right|=\mathrm{h}$ durch

$$
\gamma(\mathrm{h})=1 / 2 \operatorname{Var}\left(\mathrm{Z}\left(\mathrm{x}_{\mathrm{i}}\right)-\mathrm{Z}\left(\mathrm{x}_{\mathrm{j}}\right)\right)
$$

definiert.

Unter räumlicher Anisotropie versteht man die Richtungsabhängigkeit der räumlichen Autokorrelation. Ein Semivariogramm, bei dem ausschließlich Wertepaare in Nord-Süd-Richtung eingehen, hat beispielsweise im Fall einer entsprechenden Anisotropie andere Parameter (Range oder auch Sill und Nugget; s. S. 13) als ein solches in Ost-West-Richtung. Anisotropien können im einfachsten Fall bereits durch Koordinatentransformation aufgelöst werden.

Da in den meisten Fällen der Datenumfang zu gering ist, um für jeden möglichen Abstand h zwischen Meßstationen eine Varianz zu schätzen, werden die Abstände zunächst in Klassen (engl.: lags) zusammengefaßt. Für diese Abstandsklassen wird die Varianz, genauer die Semivarianz gemäß ( 4 ) geschätzt.

$$
\gamma(\mathrm{h})=\frac{1}{2 \mathrm{n}_{\mathrm{h}}} \sum_{\left|x_{i}-x_{j}\right|=\mathrm{h}}\left\{\mathrm{z}\left(\mathrm{x}_{\mathrm{i}}\right)-\mathrm{z}\left(\mathrm{x}_{\mathrm{j}}\right)\right\}^{2}
$$

Diese Schätzwerte aller Abstandsklassen h bilden das empirische Semivariogramm (s. Abbildung 2 für ein Beispiel). An ein solches empirisches Semivariogramm wird ein theoretisches Semivariogramm, eine Funktion angepaßt. Im Fall der vorliegenden Arbeit geschah dies mit sphärischen Modellen, die wie in ( 5 ) definiert sind.

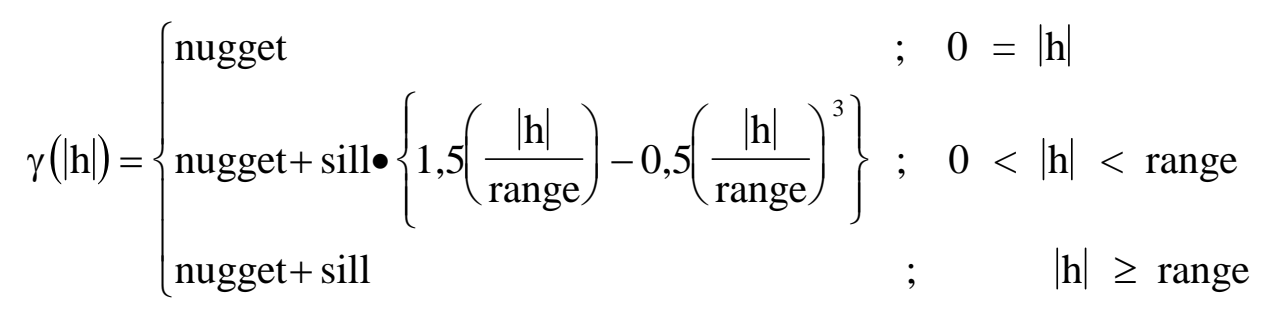


Die in ( 5 ) verwendeten Begriffe Nugget, Sill und Range sollen im folgenden Text kurz erläutert werden:

Der Nugget gibt die Varianz an, die zwischen Stationen ohne räumlichen Abstand $(h=0)$ vorliegt. Der Nugget ist somit ein Ausdruck für diejenige Streuung der Zielvariablen, die nicht durch räumliche Effekte erklärt werden kann (evtl. durch Meßfehler bedingt). Der Nugget entspricht dem Rauschen, der Grundvarianz (engl. nugget variance). Von diesem Wert aus steigt die Varianz um den Betrag des Sills bis zur sogenannten Prozeßvarianz an. Die Entfernung, für welche die Prozeßvarianz (Nugget + Sill) erreicht wird, heißt Range. Außerhalb dieses Bereiches besteht keine Korrelation zwischen den Stationen. Der Range entspricht somit dem Bereich, in dem räumliche Autokorrelation festgestellt wurde, dem Bereich, in dem Stationen mit geringem Abstand ähnlichere Werte (hier: Residuen) aufweisen als Stationen mit größerem Abstand. Über die Höhe des Sill wird quantifiziert, wieviel ähnlicher die Meßwerte naher Stationen im Vergleich zu Stationen mit großem Abstand sind.

Als Beispiel sei hier die Abbildung eines Semivariogramms angeführt, das die räumliche Autokorrelation von Residuen eines Regressionsmodells von Niederschlagswerten im Niedersächsischen Tiefland beschreibt (mittlere jährliche Niederschlagssummen zwischen 1961 und 1990, gemessen an DWD-Stationen):

Abbildung 2: Empirisches und theoretisches Semivariogramm zur Beschreibung der räumlichen Autokorrelation von Residuen eines Regressionsmodells zur Beschreibung durchschnittlicher jährlicher Niederschlagssummen im Niedersächsischen Tiefland (mittlere jährliche Niederschlagssummen zwischen 1961 und 1990)

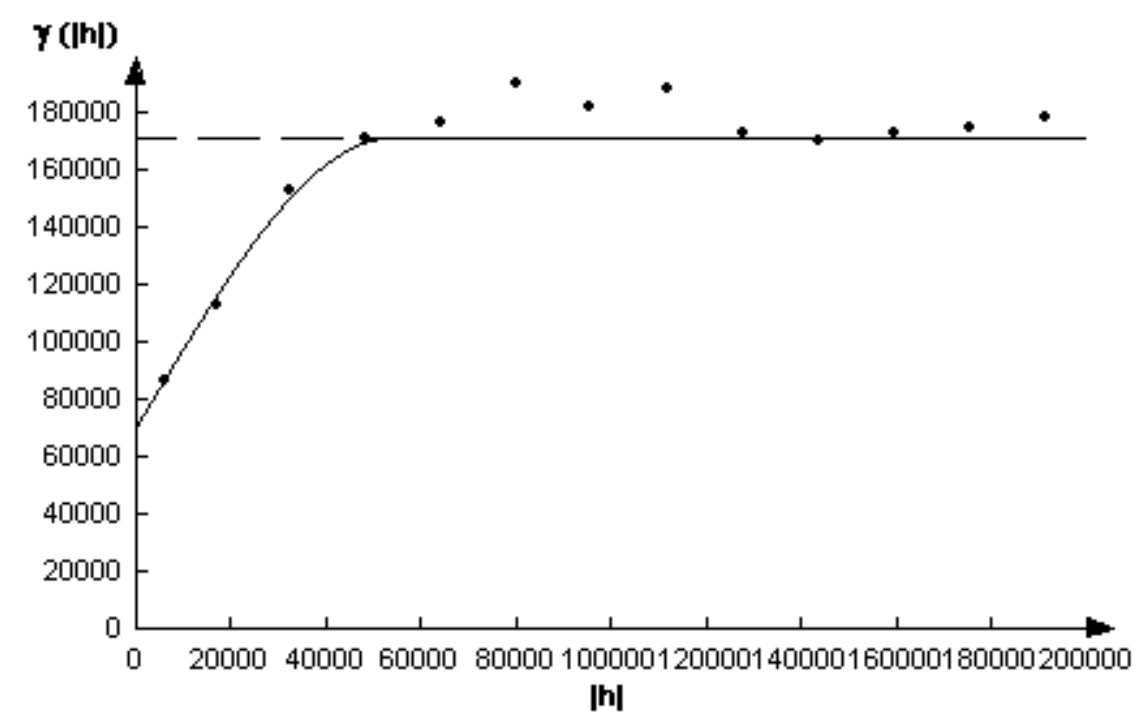


Im empirischen Semivariogramm, das in Abbildung 2 durch Punkte dargestellt ist, treten Semivarianzen über der Gesamtvarianz aller Residuen auf, was bei der Anpassung des theoretischen Variogramms jedoch allgemein keine Berücksichtigung findet. Das theoretische Variogramm wird meist nach Augenmaß angepaßt.

Das an die Daten angepaßte theoretische Semivariogramm wird zur Berechnung der Kriging-Gewichte $\lambda_{\mathrm{i}}$ herangezogen, deren Summe immer 1 sein muß. Durch diese Nebenbedingung wird eine verzerrungsfreie Vorhersage sichergestellt (RIPLEY, 1981, Kap. 4.4), wobei auch hier „Vorhersage“ nicht im zeitlichen Sinne zu verstehen ist. Der Kriging-Schätzer an einem Punkt $x, z^{*}(x)$, kann demnach wie folgt ausgehend von den $\mathrm{n}$ Stützwerten $\mathrm{z}\left(\mathrm{x}_{\mathrm{i}}\right)$ berechnet werden:

$$
\mathrm{z}^{*}\left(\mathrm{x}_{0}\right)=\sum_{\mathrm{i}=1}^{\mathrm{n}} \lambda_{\mathrm{i}} \mathrm{z}\left(\mathrm{x}_{\mathrm{i}}\right) \quad ; \sum_{\mathrm{i}=1}^{\mathrm{n}} \lambda_{\mathrm{i}}=1
$$

Die Kriging-Gewichte $\lambda_{\mathrm{i}}$ ergeben sich aus der Forderung, daß der zu erwartende quadratische Vorhersagefehler minimal sein soll $\left(E\left(z^{*}\left(x_{0}\right)-z\left(x_{0}\right)\right)^{2}=\min \right)$. Diese Forderung führt auf ein lineares Gleichungssystem ( 7 ), dessen Lösung die gesuchten Gewichte $\lambda_{\mathrm{i}}$ liefert:

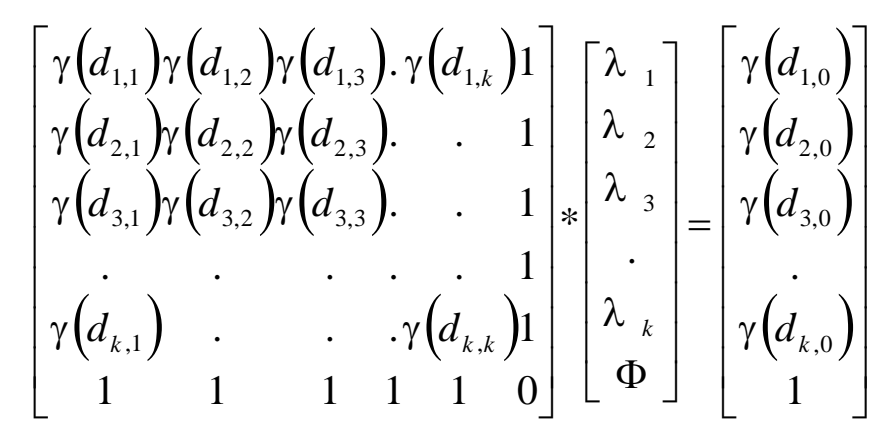

Für das Kriging-Verfahren gilt wie für andere Interpolationsverfahren, daß die Vorhersage dem dortigen Meßwert entspricht. Es können somit keine Residuen berechnet werden, über deren Größe - ähnlich zur Regression oder Varianzanalyse Aussagen über die Genauigkeit der Schätzung gemacht werden könnten. Eine entsprechende Einschätzung der Schätzgenauigkeit kann jedoch über eine Kreuzvalidierung erreicht werden: Für jeden Meßpunkt wird dabei ein KrigingSchätzwert ( $\left.\mathrm{z}^{*}\right)$ ausschließlich aus benachbarten Punkten berechnet (s. Kap. 2.5). Der Meßwert an dem jeweiligen Punkt, $z(h)$, wird nicht zur Interpolation herangezogen, sondern von dem geschätzten Wert $z^{*}$ abgezogen und man erhält eine Abweichung, die in gewissem Sinne mit dem Residuum einer Regression vergleichbar ist. Ist die Standardabweichung dieser Differenzen deutlich geringer als 
die Standardabweichung der Residuen einer Regression, so kann von einer Überlegenheit der Kriging-Schätzung gegenüber der einer (reinen) Regression ausgegangen werden.

Kriging ist nicht auf den zweidimensionalen Raum beschränkt (Entstehung durch Bergbau motiviert!). Außerdem ist es möglich, die räumliche Autokorrelation mehrerer Variablen integrativ beim sogenannten Co-Kriging zu nutzen. Die Meßwerte der Variablen können von verschiedenen Meßnetzen mit unterschiedlichem Umfang aufgenommen worden sein, wie dies typisch für Klimameßnetze ist, die Stationen mit unterschiedlichem Meßprogramm verwenden (vgl. Kap. 4.1 und ASHRAF et al., 1997). Allerdings ist die Schätzung der nötigen Semivariogramme aus den Daten entsprechend aufwendiger als bei gewöhnlichem Kriging.

HEINRICH (1994) regionalisierte für das Gebiet der damaligen 12 EG-Staaten die Klimaparameter Niederschlag und Temperatur als langjährige Durchschnittswerte. Das für geostatistische Untersuchungen untypisch große Untersuchungsgebiet und die dadurch notwendige Berücksichtigung der sphärischen Trigonometrie machten die Entwicklung eigener Software zur Modellierung der Semivariogramme und für das eigentliche Kriging notwendig.

LINDKVIST \& LINDQVIST (1997) untersuchten Strahlungsfrost in Sommernächten auf einer 9 ha großen Kahlschlagfläche in Schweden. Sie verwendeten Kriging zur flächenhaften Interpolation ihrer Messungen anhand der sie Rückschlüsse auf den Frost hervorrufende Geländeeigenschaften zogen.

JENSEN (1986) verwendete Kriging zur „Regionalisierung der Verteilungsfunktion des jährlichen Maximums des Tagesniederschlages im Kanton Zürich“. Er interpolierte Parameter von Niederschlagsverteilungen.

Das Eliminieren eines räumlichen Trends durch Regression und Kriging der Residuen wird als Universelles Kriging bezeichnet. Ein solches Verfahren wurde beispielsweise von KLEIN (1994), BANZHAF (1994), und SABOROWSKI \& STOCK (1994) zur Regionalisierung von Klimaelementen angewendet und wird in den folgenden Kapiteln behandelt.

Einen Überblick über die Theorie der Geostatistik liefern CRESSIE (1991), RIPLEY (1981) oder MATHERON (1963). Eine leichtverständliche, durch Beispiele 
unterstützte deutschsprachige Einführung ist von BILL \& FRITSCH (1996) erstellt worden.

\subsection{Berücksichtigung externer Effekte}

In den Kapiteln 2.2 und 2.3 wurde die Berücksichtigung der Lage im zwei- und dreidimensionalen Raum durch räumliche Interpolation angesprochen. In diesem Kapitel soll ein Weg zur Aufklärung desjenigen Teiles der Zielvariablenvarianz beschrieben werden, der auf weitere lageabhängige Gegebenheiten und räumliche Situationen an den Meßstellen zurückgeführt werden kann. Der Grundgedanke ist hier, daß die Ausprägung der Zielvariable an den Meßorten zumindest teilweise durch die räumliche Meßsituation bestimmt wird. Ziel muß es sein, diese Meßsituation durch geeignete räumliche Variablen $\mathrm{zu}$ beschreiben (vgl. Kap 3). Variablen zur Beschreibung der Meßsituation wie z. B. die Hangneigung können durch die Verwendung eines geographischen Informationssystems (GIS) und eines geeigneten digitalen Höhenmodells (DHM) berechnet oder auf anderem Weg hergeleitet werden (s. Kap. 3.4). Sie können als Regressoren im Rahmen einer Regressionsanalyse zur Aufklärung der räumlichen Varianz einer Zielvariablen herangezogen werden (vgl. Kap. 2.5 und Statistiklehrbücher wie HARTUNG, 1995).

Dabei muß vor allem auf die Interpretierbarkeit des Zusammenhangs der Regressoren mit der Zielvariablen geachtet werden (s.a. GÜßEFELDT, 1997). Nur der Beitrag eines interpretierbaren Regressors zur Varianzaufklärung der Zielvariablen kann auf Plausibilität überprüft werden. Nur bei plausiblen Modellen kann davon ausgegangen werden, daß das Erklärungspotential des Regressors über die konkrete Stichprobe hinaus auch für das gesamte Zielgebiet der Regionalisierung besteht.

Die Einbindung räumlicher Variablen in ein Regionalisierungsmodell richtet sich zunächst nach ihren Eigenschaften. Handelt es sich um metrische Variablen wie z. B. Geländehöhe, so kann der Zusammenhang zwischen abhängiger Variable (y) und räumlicher Variable (Regressor v) durch eine (lineare) Regression mit den Residuen $\varepsilon_{\mathrm{i}}$ beschrieben werden:

$$
\mathrm{y}\left(\mathrm{x}_{\mathrm{i}}\right)=\mathrm{b}_{0}+\mathrm{b}_{1} \mathrm{v}\left(\mathrm{x}_{\mathrm{i}}\right)+\varepsilon_{\mathrm{i}}
$$


Die Verwendung mehrerer Regressoren $v_{i}$ mit $i=1,2, \ldots, p$ führt zur Erweiterung der Regressionsgleichung:

$$
\mathrm{y}\left(\mathrm{x}_{\mathrm{i}}\right)=\mathrm{b}_{0}+\mathrm{b}_{1} \mathrm{v}_{1}\left(\mathrm{x}_{\mathrm{i}}\right)+\mathrm{b}_{2} \mathrm{v}_{2}\left(\mathrm{x}_{\mathrm{i}}\right)+\ldots+\mathrm{b}_{\mathrm{p}} \mathrm{v}_{\mathrm{p}}\left(\mathrm{x}_{\mathrm{i}}\right)+\varepsilon_{\mathrm{i}}
$$

Die Parameter der Regressionsgleichungen $b_{0}, b_{1}, \ldots, b_{p}$ wurden im Rahmen der vorliegenden Arbeit durch die gewöhnliche Methode der kleinsten (Abweichungs-) Quadrate bestimmt (ordinary least squares = OLS; s. z. B. HARTUNG, 1995).

VENT-SCHMIDT (1985) erstellte mit Hilfe eines Regressionsmodells rasterorientierte Karten zur Verteilung der mittleren Lufttemperatur in Nordhessen. Angaben zur Repräsentanz der Meßstationen für das Untersuchungsgebiet sind auf die Verteilung der Geländehöhe beschränkt. Durch die Verwendung eines Höhengradienten konnten bereits $79 \%$ der Varianz der mittleren Temperaturen erklärt werden. Weitere $6 \%$ konnten durch Gradienten in Nord-Süd und Ost-West Richtung erklärt werden. Er konnte nicht auf ein spezielles GIS zurückgreifen und schätzte den Zielwert für jeden Rasterpunkt aus dem Meßwert der nächstgelegenen Meßstation und die Gradienten und den Lageunterschied zu dieser Meßstation. Diese Vorgehensweise fand in ähnlicher Weise auch bei STEINER \& LEXER (1998) Verwendung.

Für sein Untersuchungsgebiet Zentral- und Hochasien ermittelte BÖHNER (1996) für 20 Untergebiete (8 bis 15 Meßstationen) mit potentiell homogenen Vertikalgradienten lineare Regressionsmodelle mit den Koordinaten und der Geländehöhe der Meßstationen als Regressoren. Für das ,arithmetische Mittelzentrum“ eines jeden Untergebietes berechnete er sodann einen „Basiswert“ anhand der Regressionskonstante und der horizontalen Gradienten. Das regionalisierte Element (durchschnittliche Temperatur und durchschnittliche Niederschlagssumme) wurde sodann an jedem Rasterpunkt (7,3 km $\times 7,3 \mathrm{~km})$ durch eine Dreiecksinterpolation der Basiswerte und der Vertikalgradienten der nächstgelegenen drei arithmetischen Mittelzentren berechnet. Eine solche Dreiecksinterpolation -allerdings ohne vorherige Unterteilung in homogene Untergebiete - verwendeten auch BURGA \& PERRET (1998) für die Regionalisierung der Jahresmitteltemperatur ausgehend von 36 Meßstationen in der Schweiz. Dieses Verfahren bietet wie das im Zusammenhang mit der Arbeit von VENTSCHMIDT (1985) dargestellte Verfahren den Vorteil einer exakten Schätzung an den Meßstellen. Allerdings wird dadurch eine Abschätzung des Regionalisierungsfehlers erschwert. Der Regressionsfehler kann bei BÖHNER (1996) nicht als Schätzer für den 
Regionalisierungsfehler verwendet werden, da die Interpolation von Regressionsergebnissen zwischen drei nächstgelegenen Stationen bzw. Mittelzentren $\mathrm{zu}$ anderen Ergebnissen führt als eine Anwendung des gefundenen Regressionsmodells für das gesamte Untersuchungs- oder Untergebiet. Zudem erscheint die bei BÖHNER (1996) durchgeführte lineare Interpolation zwischen Zentren homogener Untergebiete nicht konsequent. Wenn homogene Untergebiete ganz im Sinne herkömmlicher Regionalisierung gefunden und ausgeschieden wurden, sollten zur Regionalisierung in diesen Untergebieten nicht auch die Gebietsmittel benachbarter und damit andersartiger Stationskollektive berücksichtigt werden. Eine konsequente Unterteilung in Untergebiete erfordert jedoch die Festlegung von Grenzen und führt bei der Darstellung der Ergebnisse eventuell zu erheblichen Differenzen der Werte der regionalisierten Größe beiderseits der Grenzen. Eine Möglichkeit zur Behandlung dieser Problematik wird im Rahmen dieser Arbeit vorgestellt.

FELBERMEIER (1994) verwendete zur Regionalisierung von Niederschlag und Temperatur durch Regressionsanalyse geomorphologische Variablen als Regressoren. Er ließ in eine schrittweise Regressionsanalyse zusätzlich Transformationen (Potenzen) dieser Variablen und Kombinationen mehrerer Variablen eingehen. Die Verwendung von Transformationen und Kombinationen räumlicher Variablen schränkt die Interpretierbarkeit der Modelle ein, Plausibilität und Einfachheit der Modelle werden zugunsten der Bestimmtheitsmaße (Präzision) eingeschränkt. Die Unsicherheiten der Werte der verwendeten Variablen durch die Lagebestimmung der Niederschlagmeßstationen mit $\pm 1 \mathrm{~km}$ müssen dabei als hoch eingeschätzt werden (vgl. Kap. 3.1). LENZ et al. (1996) führen Probleme bei der Regression von Depositionsanreicherungsfaktoren auf die unbefriedigende Genauigkeit der von ihnen verwendeten räumlichen Variablen zurück.

BlenNOW \& PERSSON (1998) konnten standardisierte Temperaturmessungen in einem Untersuchungsgebiet von ca. $3000 \mathrm{~m} \times 3000 \mathrm{~m}$ durch die Regression mit den räumlichen Variablen Geländehöhe, Höhendifferenz zum Minimum in einer Umgebung von 200 m Radius (relative Höhe), sky view factor und Vorkommen von Moorböden regionalisieren. Die Messungen fanden in fünf klaren und windstillen Nächten im Sommer des Jahres 1991 zwischen Mitternacht und 2 Uhr morgens statt. Die Regressionskoeffizienten der Geländehöhe und der Differenz zum 
Umgebungsminimum haben positive Werte, die auf Inversionslagen zurückgeführt werden, wie sie unter den beschriebenen Umständen zu erwarten sind. Negative Regressionskoeffizienten für den sky view factor und Moorböden belegen die erhöhte nächtliche Abkühlung durch Abstrahlung bzw. durch entsprechende Bodenbedingungen. In Bezug auf den Einfluß von Moorböden fehlt jedoch eine griffige physikalische Erklärung für dieses Phänomen. Der lineare Regressionsansatz bietet eine gute Interpretationsmöglichkeit der Ergebnisse. Durch die Beschränkung auf eine bestimmte Wettersituation und Jahreszeit sind die Ergebnisse nicht vergleichbar mit denen von Regionalisierungen langjähriger Mittelwerte, die Ergebnis vieler unterschiedlicher Wettersituationen und somit Ausdruck des örtlichen Klimas sind.

LAUGHLIN \& KALMA (1990) kamen bei der Regionalisierung von Minimumtemperaturen zu dem Ergebnis, daß die Veränderung der Lufttemperatur mit der Geländehöhe durch die mittlere nächtliche Windgeschwindigkeit, den nächtlichen Energieverlust durch Strahlung und die Temperatur auf einer hochgelegenen Temperaturmeßstation modelliert werden kann. Das Risiko von Nachtfrösten zeigte außerdem eine hohe Korrelation mit der räumlichen Variable ,flowaccumulation“, welche der hier verwendeten Variable Kaltluftabflu $\beta$ ähnelt.

Eine bisher nicht erwähnte Problematik entsteht bei der Einbindung von klassifizierenden Variablen. Eine direkte Einbindung in ein Regressionsmodell ist nicht möglich. Das Erklärungspotential einer Klassifikationsvariable wie z. B. der Landnutzungsform kann jedoch durch eine Varianzanalyse (HARTUNG, 1995) oder durch die Verwendung von Dummy-Variablen (vgl. JANSEN et al., 1997, oder BlenNOW \& Persson, 1998) genutzt werden. Durch die Datenhaltung in einem Geographischen Informationssystem (GIS; ARC/Info) ist es möglich, die Klassen eines Landnutzungs-Rasters in verschiedene metrische Variablen zu überführen (Kap. 3.4.1), wie dies auch von GoßMANN et al. (1993) beschrieben und von GÜßEFELDT (1997) erwähnt wird.

Wie schon in Kap. 2.3 zum Thema „Kriging“ angedeutet wurde, ist es möglich, die Residuen von Regressionen durch eine geostatistische Analyse auf räumliche Autokorrelation zu überprüfen (Variogrammschätzung) und diese durch Universelles Kriging zur Verbesserung der Schätzung zu nutzen. 
So wird im Freiburger Regionalisierungsmodell FREIM (vgl. GOßMANN et al., 1993, GÜßEFELDT, 1997, KLEIN, 1994, oder BANZHAF, 1994) Universelles Kriging zur Regionalisierung von Klimaelementen verwendet. Ebenso wie von SABOROWSKI \& STOCK (1994) wurde mit dem Kriging eine Verbesserung von durch Regression gefundenen Modellen angestrebt. Letztere Arbeit konnte zudem zeigen, daß durch iterative Anpassung eines Semivariogramms (Kap. 2.5) die Qualität der Schätzung auf plausiblem Weg weiter verbessert werden kann.

\subsection{Beschreibung der angewandten Methodik}

In den Kapiteln 2.3 und 2.4 wurden bereits zwei statistische Methoden vorgestellt, die im Rahmen der hier vorgestellten Arbeit zur Erklärung der räumlichen Verteilung von Zielvariablen herangezogen wurden: Die schrittweise lineare Regression und das geostatistische Verfahren Kriging. Gegenstand der Beschreibung des jeweils angepaßten Modells sind die Korrelation der Zielvariablen mit räumlichen Variablen (Regression) bzw. die räumliche Autokorrelation der Zielvariablen bzw. der Regressionsresiduen (Kriging). In diesem Kapitel sollen sowohl die Statistiken vorgestellt werden, die zur Beurteilung der Modelle dienten als auch die Vorgehensweisen, welche die Plausibilität und Einfachheit der Modelle sicherstellen sollten. Der Systematik zur Regionalisierung entsprechend wird hier zunächst auf die Regression, dann erst auf das Kriging eingegangen.

Die schrittweise Regressionsanalyse ist eine oft verwendete Methode, um Zusammenhänge zwischen einer räumlich verteilten Zielvariablen und geomorphometrischen Variablen zu beschreiben (vgl. BlenNow \& PERSSON, 1998, FElBermeIER, 1994, KleIN, 1994, oder BANZHAF, 1994). Die Methodik der partiellen Pfadanalyse, wie sie von GÜßEFELDT (1997) beschrieben und beispielsweise von KLEIN (1994) und BANZHAF (1994) angewendet wurde, berücksichtigt dabei die Multikollinearität der Regressoren und eliminiert diese vor der Hinzunahme neuer Variablen. Der Aspekt der Multikollinearität fand im Rahmen der vorliegenden Arbeit keine gesonderte Berücksichtigung. Bei stark korrelierten 
Regressoren wurde jedoch die gemeinsame Integration in Regressionsmodellen vermieden (vgl. Geländehöhe und Lee-Effekt 50 in Kap. 5.1.2.1).

Zur Vermeidung allzu großer Probleme durch Interaktionen zwischen den Regressoren und zur Sicherstellung übersichtlicher und interpretierbarer, möglichst einfacher Modelle wurde die Zahl der verwendeten Variablen durch eine Bedingung gering gehalten. Ausschließlich solche Variablen wurden im Zuge der schrittweisen linearen Regression in die Modelle aufgenommen, die das Bestimmtheitsmaß des Modells um mindestens $5 \%$ erhöhen konnten und zumindest nominell auf dem $5 \%$ Niveau signifikant waren (Kriterium: Präzision). Zudem mußte der für die Variable bestimmte Regressionskoeffizient mit bekannten physikalischen Zusammenhängen mit der Zielvariablen vereinbar sein (Kriterium: Plausibilität). Dies entspricht der von FRÄNZLE et al. (1989) gemachten Aussage, daß sich für die Prädiktorvariablen nur herausfinden läßt, ,,welche von ihnen mit der Zielvariablen am engsten verknüpft ist. Ob dieser Zusammenhang sachlich etwas erklärt oder nicht, ist für die jeweilige Problemstellung vor dem Hintergrund des vorhandenen Wissens zu beurteilen.“ Auch GÜßEFELDT (1997) fordert eine am Wissen orientierte Vorauswahl möglicher Prädiktoren.

Die Wahl eines derart vorsichtigen Modellierungsansatzes schien in Anbetracht des explorativen Charakters einer schrittweisen Regression angebracht. Die schrittweise lineare Regression mit den beiden oben aufgeführten Bedingungen der Präzision und Plausibilität führte durchweg zu Modellen mit nicht mehr als vier Regressoren (s. Kap. 5). Neben der Einfachheit der Modelle sollte durch die $5 \%$-Bedingung auch die Gültigkeit der in den Daten gefundenen Zusammenhänge für das gesamte Zielgebiet gewahrt bleiben. Mit der Hinzunahme von weniger bedeutenden Variablen oder Transformationen bereits integrierter Variablen steigt das Risiko einer Überinterpretation der untersuchten Daten unverhältnismäßig stark im Vergleich zur erzielten Verbesserung der Präzision des Modells. Einfachheit und Plausibilität der Modelle sind somit eine wichtige Voraussetzung für die Verwendbarkeit und Akzeptanz der Regionalisierungsergebnisse.

Um die Gültigkeit der in den Daten vorhandenen, durch die Regressionsmodelle beschriebenen Zusammenhänge für das Zielgebiet $\mathrm{zu}$ überprüfen, wurde eine Repräsentanzuntersuchung der zugrunde liegenden Datenkollektive durchgeführt. 
Die Ergebnisse werden in Kap. 4 im Zuge der Beschreibung der verwendeten Datenkollektive - im Fall der Depositionsdaten gesondert in Kap. 4.2.3 - dargestellt.

Die Präzision der Modelle wurde über das Bestimmtheitsmaß und die Wurzel des mittleren quadrierten Regressionsfehlers (rooted mean squared error, RMSE) beurteilt. Das Bestimmtheitsmaß entspricht dem Anteil der erklärten Varianz an der Gesamtvarianz der Zielvariablen (s. ( 10 )) und stellt so ein relatives Maß für die Anpassung eines Modells an die Daten dar. Es ist unabhängig von Maßeinheiten und kann so auch zum Vergleich von Modellen ganz unterschiedlicher Zielvariablen (z. B. Niederschlagssumme und durchschnittliche Lufttemperatur) herangezogen werden.

$\mathrm{R}^{2} \quad-$ Bestimmtheitsmaß

$$
R^{2}=\frac{\sum\left(Y_{i}-\bar{y}\right)^{2}}{\sum\left(y_{i}-\bar{y}\right)^{2}}
$$

$\mathrm{Y}_{\mathrm{i}} \quad$ - Modellwerte

$\bar{y} \quad$ - arithmetisches Mittel der Meßwerte

$\mathrm{y}_{\mathrm{i}} \quad-$ Meßwerte

Der RMSE, definiert durch

$$
\mathrm{RMSE}=\sqrt{\frac{\mathrm{SQR}}{\mathrm{DF}}}=\sqrt{\frac{\sum\left(\mathrm{r}_{\mathrm{i}}\right)^{2}}{\mathrm{n}-\mathrm{k}-1}}
$$

r $\quad-$ Residuum

n - Anzahl der Beobachtungen (Zahl der verwendeten Stationen)

k - Anzahl der im Modell verwendeten Regressoren,

ist ein Maß für die unerklärten Abweichungen der Meßwerte vom Regressionsmodell, die Residuen. Diese Residuen sind bei geeigneter Datenbasis auch Grundlage einer anschließenden geostatistischen Analyse (vgl. Kap. 2.3).

Eine zwei- oder dreidimensionale graphische Darstellung der räumlichen Lage der Residuen sollte auf die räumliche Autokorrelation aufmerksam machen, sofern sie denn in den Daten (hier: Residuen) vorhanden ist. Sie zeigt sich durch eine größere Ähnlichkeit der Werte (geringere Differenzen) von nahe beieinander liegenden Punkten im Vergleich mit solchen, deren räumliche Distanz größer ist. Als Beispiel einer solchen zweidimensionalen Betrachtung seien hier die Residuen eines Regressionsmodells der langjährigen mittleren Jahresniederschlagssumme im niedersächsischen Tiefland dargestellt (Abbildung 3), die auch Grundlage für das 
bereits in Abbildung 2 dargestellte empirische Semivariogramm sind. Die ganzzahligen Residuen wurden zur graphischen Darstellung in 5 Größenklassen gleicher Häufigkeit eingeteilt (52 Residuen pro Klasse). Die in Abbildung 3 dargestellten Residuen zeigen eine kleinräumige Ähnlichkeit, wie sie bei räumlicher Autokorrelation zu erwarten ist:

Abbildung 3: Zweidimensionale räumliche Darstellung der Residuen des Regressionsmodells für die durchschnittliche jährliche Niederschlagssumme im niedersächsischen Tiefland, eingeteilt in 5 Klassen gleicher Häufigkeit

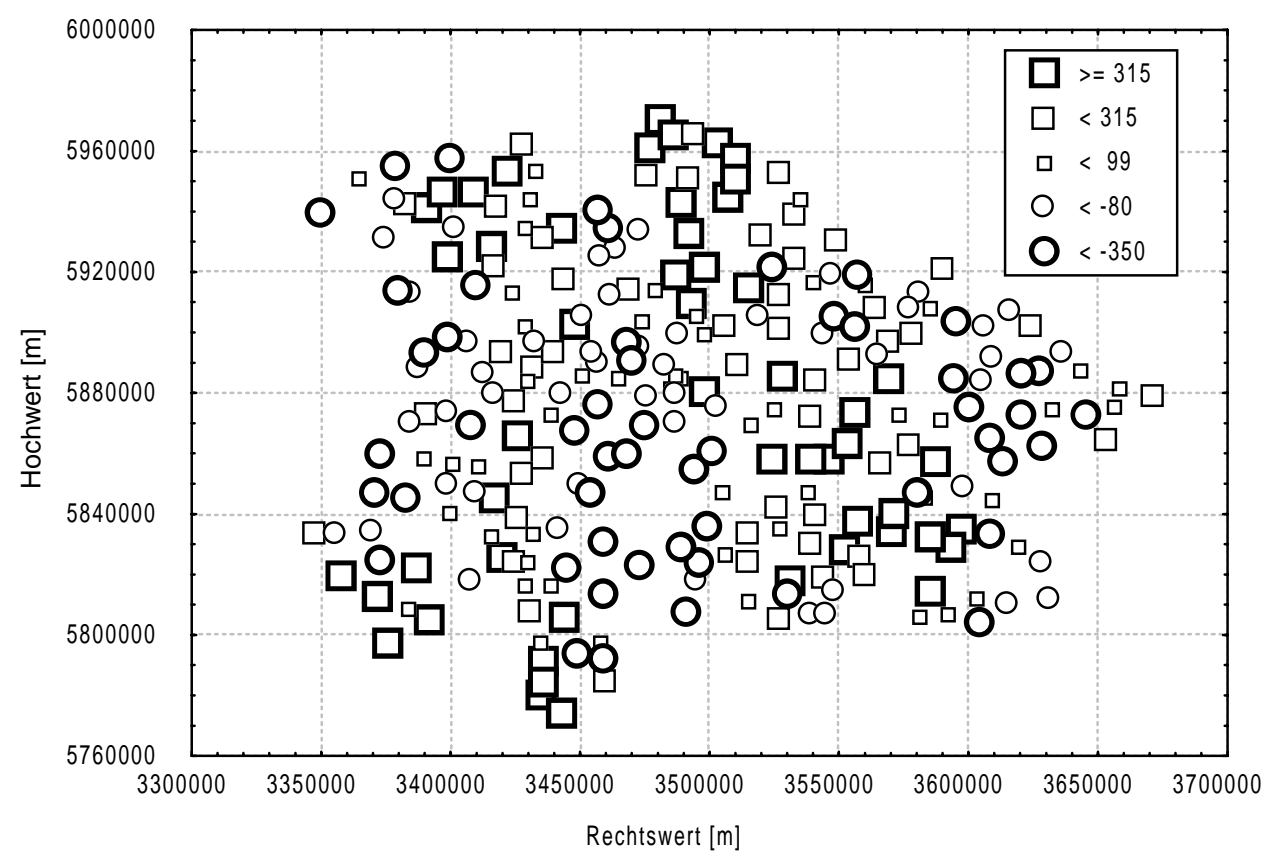

Eine solche Darstellung kann mit Hilfe des SAS-Statistik-Programmpakets oder mit einem anderen Programm erstellt werden (Abbildung 3 erstellt mit EXCEL 97). Das zur Semivariogrammanpassung verwendete Programmpaket VARIOWIN 2.2 liefert diese Darstellungsmöglichkeit nicht. Über die Kombination von Variogrammwolken (Quadrierte Meßwertdifferenz über Abstand h) und Lagekarten der Ausgangsdaten können jedoch Besonderheiten in der räumlichen Struktur der Daten analysiert werden (vgl. Kap. 6.1.2.1, S. 156). Auch eine Variogrammoberfläche (,variogram surface“) und die Darstellung des aus den Daten direkt berechneten empirischen Semivariogramms sind geeignete Hilfsmittel, um eventuelle Abweichungen von der ansonsten vorausgesetzten Bedingung der Stationarität zu analysieren.

Hierzu sei in Abbildung 4 ein Beispiel gegeben. Datengrundlage sind erneut die Residuen des linearen Regressionsmodells des langjährigen mittleren jährlichen Niederschlagssumme im niedersächsischen Tiefland. 


\section{Abbildung 4: Fenster zur Analyse und Beschreibung räumlicher Autokorrelation mit dem Programmpaket VARIOWIN 2.2 am Beispiel der langjährigen mittleren jährlichen Niederschlagssumme im niedersächsischen Tiefland}

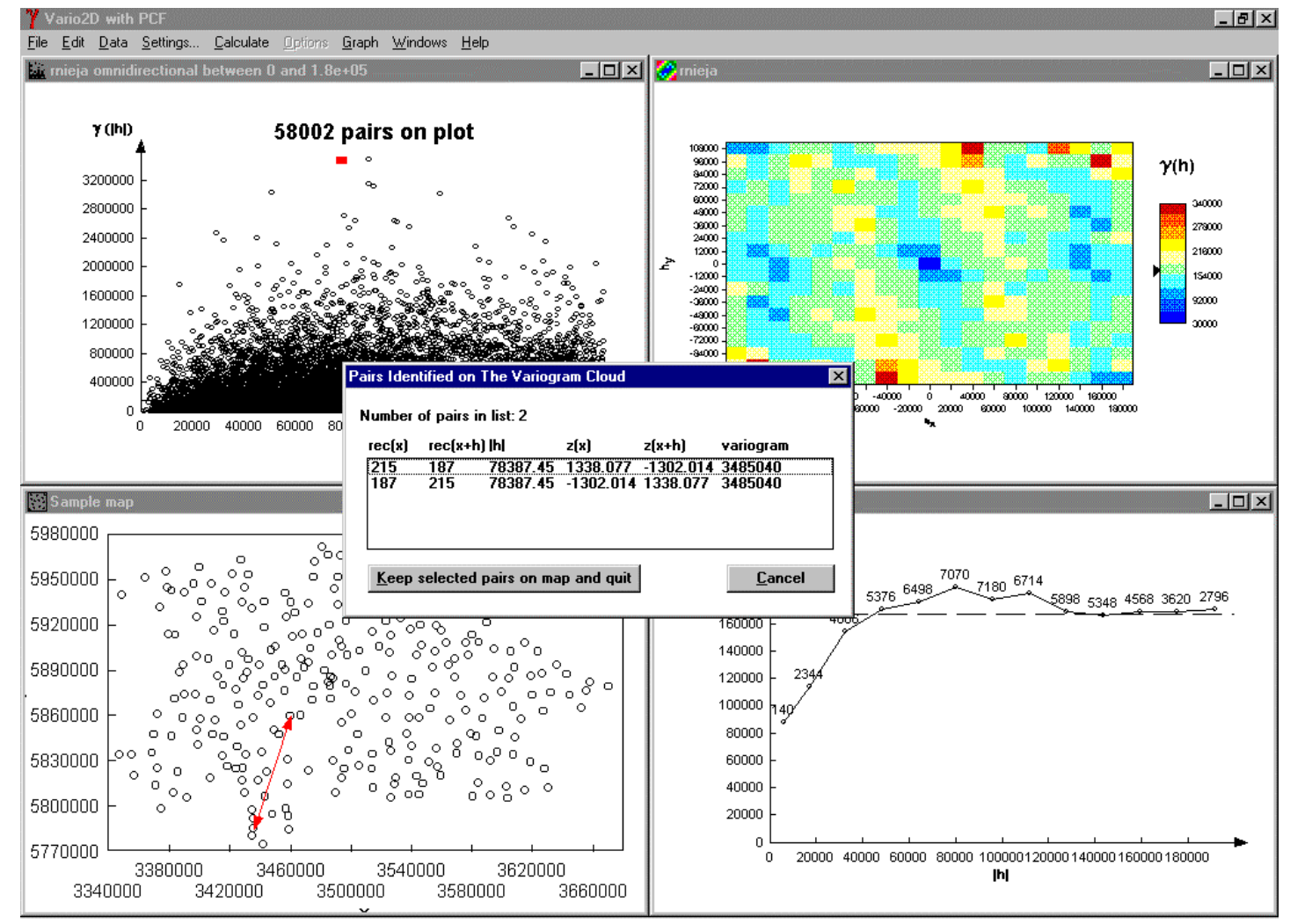

Oben links ist die Variogrammwolke, darunter die Lagekarte der Meßstationen angeordnet. Oben rechts befindet sich die Variogrammoberfläche über der Darstellung des empirischen Semivariogramms. Auffällig am SemivariogrammVerlauf ist die relativ hohe Semivarianz im Bereich von ca. 50 bis $110 \mathrm{~km}$ räumlicher Distanz $|\mathrm{h}|$, die über die gestrichelt dargestellte Gesamtvarianz hinausgeht (s.a. Abbildung 2). Diese untypische, jedoch häufig anzutreffende Beobachtung kann in diesem Fall durch hohe Semivarianzen in Richtung NNO-SSW (Nordnordost nach Südsüdwest) in der Darstellung der Variogrammoberfläche erklärt werden. Diese sind auch in Form einiger hoher Semivarianzen in diesem Bereich in der Semivariogrammwolke ${ }^{1}$ zu erkennen. Mit Hilfe der Lagekarte können diese räumlich fixiert werden und es bestätigt sich, daß fast alle hohen Semivarianzen in diesem Abstandsbereich in Richtung NNO-SSW bzw. NO-SW liegen. Die Variogrammwolke zeigt deutlich, daß diese Abweichungen nur auf einzelne

\footnotetext{
${ }^{1}$ Die Begriffe Semivariogramm und Variogramm werden hier wie so oft in der Literatur synonym verwandt, mathematisch korrekt ist der Begriff Semivariogramm.
} 
Beobachtungen zurückzuführen sind. Daher wird diese Abweichung nicht als gewichtige Verletzung der Stationaritäts-Voraussetzung angesehen. Der beobachtete Effekt liegt zudem außerhalb des Bereiches, für den im theoretischen Semivariogramm die räumliche Autokorrelation beschrieben wird (vgl. Abbildung 2). Im Bereich des Range wurden keine bedeutenden Unregelmäßigkeiten festgestellt.

Grundvoraussetzung für eine geostatistische Analyse ist in jedem Fall eine ausreichend umfangreiche Datenbasis, die zudem auf geeignete Weise räumlich verteilt sein muß. Über beide Kriterien können die in Abbildung 4 dargestellten VARIOWIN-Fenster Auskunft geben. So sind die aus den Daten berechneten Werte des empirischen Semivariogramms mit der Zahl der zugrundeliegenden Wertepaare versehen. Diese Zahl der Wertepaare in einem Distanzschritt des empirischen Semivariogramms sollte grundsätzlich nicht unter 50 liegen (JOURNEL \& HuiJBREGTS, 1978 aus: HeINRICH, 1994), im schlechtesten Fall sollten für jede Schätzstelle des empirischen Semivariogramms mindestens 20 Wertepaare zur Verfügung stehen (SABOROWSKI, mdl. Mitteilung 1996). Um dies auch für geringe räumliche Distanzen erfüllen zu können, muß die Datenbasis nicht nur umfangreich sein, auch müssen Meßstellen zumindest kleinräumig in größerer Dichte vorkommen (Meßnetzverdichtung). Nach BERKE (1998, S. 132) sind flächendeckende Verteilungen mit Meßnetzverdichtungen für eine „geostatistische Analyse von Vorteil“. Im Rahmen der vorliegenden Arbeit erfüllten nur die Niederschlagsdatenkollektive die Bedingungen für eine geostatistische Analyse (Kap. 4.1.3), da sie sowohl den erforderten Datenumfang als auch eine geeignete flächendeckende Verteilung mit Meßnetzverdichtungen aufwiesen.

Zur Anpassung theoretischer Variogrammodelle wurden ausschließlich sphärische Modelle verwendet (s. ( 5 ) auf S. 12 und Abbildung 2). Dies konnte komfortabel in einem speziellen Modul von VARIOWIN 2.2 erledigt werden. Das Kriging mit den so angepaßten Semivariogrammodellen wurde mit dem Geostatistik-Programmpaket GSTAT (s. PEBESMA, 1997) durchgeführt. Dieses arbeitet auch unter UNIX und hat eine Schnittstelle zu dem hier verwendeten GIS ARC/Info. Beides ermöglichte die volle Ausnutzung der zur Verfügung stehenden Soft- und Hardware, wodurch eine zufriedenstellende Arbeitsgeschwindigkeit erreicht werden konnte. 
Der ersten Semivariogrammanpassung an die Residuen des schrittweise bestimmten OLS-Regressionsmodells (ordinary least squares) folgte ein iteratives Verfahren zur Modelloptimierung, wie es bei SABOROWSKI \& STOCK (1994) beschrieben wurde. Es ist möglich, daß ein Teil der räumlichen Autokorrelation durch die Regression (OLS) überdeckt wird. Die Regression erklärt einen größeren Teil der Zielvariablenvarianz durch die Regressoren als tatsächlich auf deren Varianz zurückzuführen ist. Das an die Residuen der OLS-Regression angepaßte Semivariogramm dient im iterativen Verfahren zur Entwicklung einer Covarianzmatrix, die im nächsten Schritt in einer sogenannten generalisierten kleinste Quadrate Schätzung (generalized leased squares, GLS), in einer zweiten Regression integriert wird. Von den Residuen dieser ersten GLS-Schätzung läßt sich ein zweites Variogramm schätzen und so fort bis 2 aufeinanderfolgende Variogramme mit den gleichen Parametern (Nugget, Sill und Range) angepaßt werden. Das Regionalisierungsmodell wird letztlich durch die Parameter der GLS-Regression und die Parameter der letzten Semivariogrammanpassung definiert (s. Tabelle 50 und Tabelle 56). Zur Berechnung der GLSModelle wurde ein FORTRAN-Programm unter DOS verwendet, das freundlicherweise von Prof. Dr. Saborowski zur Verfügung gestellt wurde. Auch mit GSTAT (s. PEBESMA, 1997) ist diese Berechnung nach kurzer Einarbeitungszeit problemlos zu leisten.

Die Verbesserung der Schätzung, die durch ein solches iteratives Verfahren (OLS Semivariogramm - GLS - Semivariogramm - GLS - usw.) erreicht wird, kann mit Hilfe einer Kreuzvalidierung (Kap. 2.3, Seite 14) quantifiziert werden. Zieht man von der Standardabweichung der OLS-Residuen res $\left(\mathrm{x}_{\mathrm{i}}\right)$ die Standardabweichung der Differenzen zwischen den Kriging Schätzwerten $z^{*}\left(x_{i}\right)$ und den Meßwerten $z\left(x_{i}\right) a b$, so erhält man ein Maß für die Verringerung des mittleren quadrierten Fehlers in der Maßeinheit der abhängigen Variablen (dSTD, vgl. Tabelle 51 und Tabelle 57). Alle hierzu nötigen Standardabweichungen werden durch das Kreuzvalidierungs-Modul XValid des Geostatistik-Programmpakets GEOEAS berechnet. Hierzu ist lediglich die Angabe der Parameter des letzten Semivariogrammodells und die in dem letzten iterativen Schritt mit VARIOWIN 2.2 erzeugte PCF-Datei (pair comparison file) notwendig.

Sofern es zu Abweichungen von der in diesem Kapitel vorgestellten Methodik kam, wird das in den entsprechenden Kapiteln erwähnt (s. Kap. 4.2.4, „Besonderheit der Regionalisierung der Depositionen“). Allgemein wurden die schrittweisen linearen 
Regressionen mit dem Statistik-Programmpaket SAS 6.03 bzw. SAS 6.11 durchgeführt. Das GIS ARC/Info wurde zur Modellierung und Bereitstellung der räumlichen Variablen verwendet. Graphische Darstellungen der gefundenen Zusammenhänge wurden mit dem unter WINDOWS komfortableren StatistikProgrammpaket STATISTICA 5.1 oder mit EXCEL 97 erstellt. Die Umsetzung der Regionalisierungsmodelle zur kartographischen Darstellung der Ergebnisse erfolgte mit dem GIS ARC/Info. 


\section{Räumliche Variablen zur Beschreibung der Meßsituation}

Wie in Kapitel 2.4 beschrieben, wurden die Varianzen der Zielvariablen, wie z. B. Lufttemperatur und Niederschlag, so weit als möglich durch die Varianz räumlicher Variablen aufgeklärt. Der Erfolg dieser linearen Trendaufklärung ist dabei abhängig von der Korrelation zwischen der Zielvariablen und den räumlichen Variablen und zwischen den räumlichen Variablen unter sich. Neben dem Ziel der Präzision der Modelle wurde vor allem auch Wert auf die Plausibilität derselben gelegt (vgl. Kap. 2.5). Hierzu war es notwendig, räumlich verteilte Variablen zu ermitteln und zu definieren, die ein Phänomen in der Natur beschreiben, das in kausalem Zusammenhang mit der Ausprägung der Zielvariablen steht. Nur solche Variablen fanden Verwendung, für die ein physikalischer Einfluß auf die Zielvariable plausibel erscheint. So wird im allgemeinen eine Abnahme der durchschnittlichen Lufttemperatur mit zunehmender Geländehöhe erwartet (vgl. z. B. HäCKEL, 1990 oder GEIGER, 1961).

Eine genaue Verortung der Meßstationen ist die Grundlage für die exakte Ermittlung der dort gültigen Variablenwerte. Die Größenordnung möglicher Fehler durch ungenaue Verortung von Meßstationen soll in Kap. 3.1 vorgestellt werden. Im Zuge der Beschreibung der verwendeten Datenkollektive (Kap.4) werden die jeweiligen Schwierigkeiten bei der Verortung der Meßstationen gesondert besprochen.

\subsection{Fehler durch ungenaue Verortung der Meßstationen}

Die in diesem Kapitel getroffenen Aussagen zur Verortung der Meßstationen werden am Beispiel der Variablen Geländehöhe und Hangneigung erläutert, deren Definitionen allgemein bekannt sein dürften. 
Nach Definition und Berechnung lagen die räumlichen Variablen grundsätzlich als Rasterdateien im GIS vor. Die Zuordnung von Rasterwerten zu den Meßstationen erfolgte im GIS ARC/Info mit dem Befehl <latticespot> auf bilinearem Wege (s. ESRI, 1995).

Es ist einleuchtend, daß bereits durch diese erste bilineare Interpolation Fehler bzw. Abweichungen zwischen dem aus dem Raster ermittelten Wert und dem realen Wert der Variablen am Zielort entstehen können. Diese sollten aber bei der grundsätzlich verwendeten Rasterweite von 50 m relativ gering sein. Der Fehler, der hier betrachtet werden soll, ist der zusätzlich durch ungenaue Verortung der Stationen mögliche Fehler.

Als Größe für den Verortungsfehler wurden die Standardabweichung der Rasterwerte von Geländehöhe und Hangneigung in kreisförmigen Umgebungen jedes Rasterpunktes mit den Radien 50, 200, 500, 1000, 1500 und 2000 m ermittelt (ARC/Info-Funktion: <focalstd>).

Die für die Meßstationen mit bilinearer Interpolation aus den StandardabweichungsRastern ermittelten Werte wurden gemittelt und in Abbildung 5 getrennt für Bergund Tiefland in Niedersachsen dem Radius der jeweiligen zugrunde liegenden Umgebung gegenübergestellt. Diese Darstellungsweise gibt einen Eindruck von der Unsicherheit der Werte der beiden räumlichen Variablen an den Meßstationen. Je ungenauer die Verortung der Meßstationen, desto unsicherer ist auch die Beschreibung der Meßsituation durch die Variablen.

Bereits relativ geringe Fehler der Verortung führen im Fall der Hangneigung zu relativ großen Fehlern im Vergleich mit der Standardabweichung der für die Meßstationen ermittelten Werte (7.3\% für das Bergland und $1.7 \%$ für das Tiefland). Dies zeigt die größere Anfälligkeit der Variable Hangneigung im Verhältnis zur Geländehöhe. Die für die Geländehöhe ermittelten Standardabweichungen in den Umgebungen liegen weit unter den Standardabweichungen der an den Meßstationen ermittelten Werte (170.1 m im Bergland und $28.4 \mathrm{~m}$ im Tiefland).

Neben dem Unterschied zwischen der Geländehöhe und der Hangneigung springt der Unterschied zwischen Berg- und Tiefland ins Auge. Die höhere Reliefenergie im niedersächsischen Bergland führt deutlich zu höheren Standardabweichungen. 
Abbildung 5: Mittlere Standardabweichungen von Rasterwerten der Geländehöhe und der Hangneigung in kreisförmigen Umgebungen mit Radien von 50 bis 2000 m um 128 DWD-Berglandstationen bzw. 260 DWD-Tieflandstationen

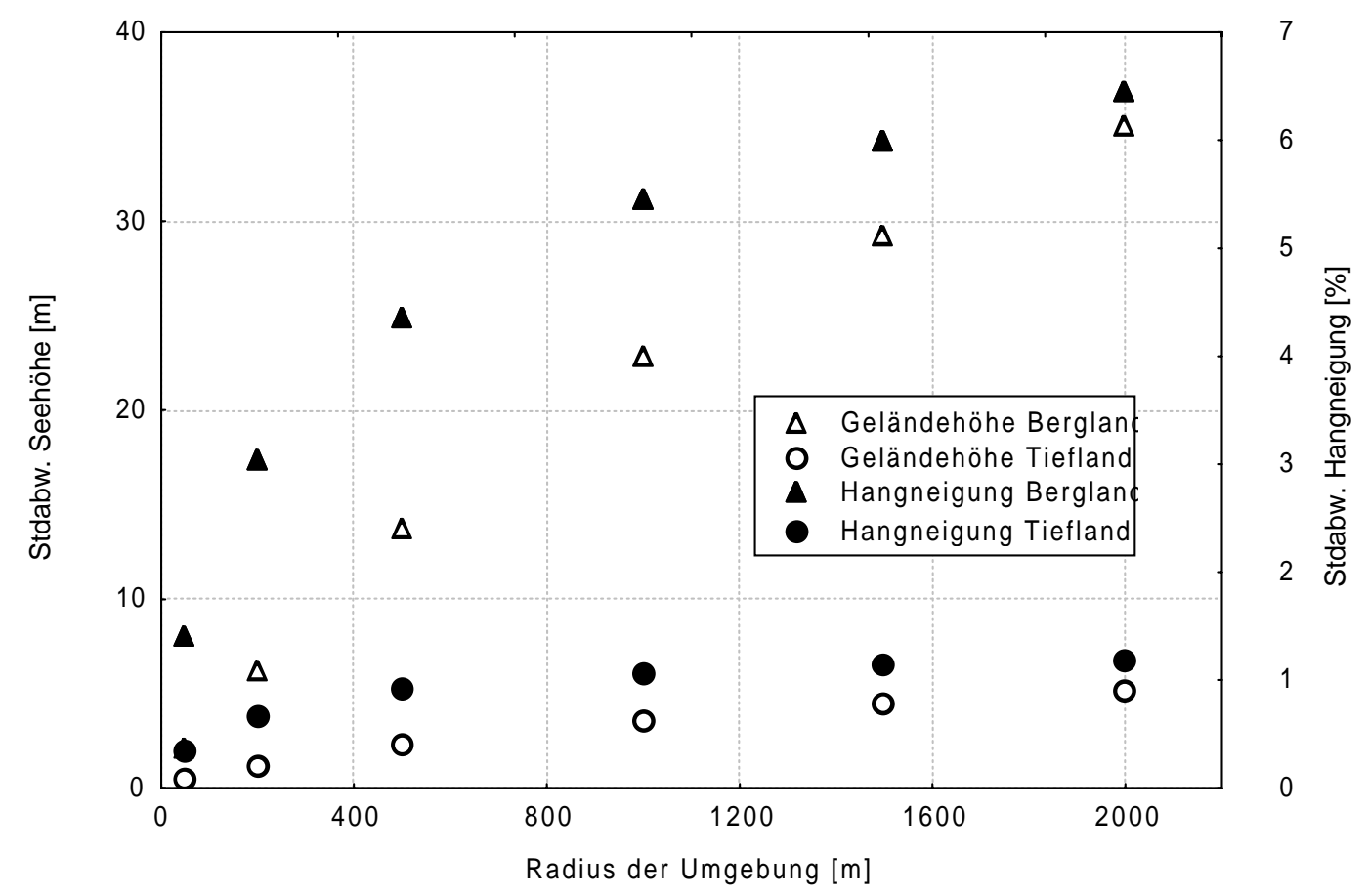

\subsection{Lageabhängige Variablen}

\subsubsection{Koordinaten}

Die geographische Lage von Meßstationen wurde im Rahmen der vorliegenden Arbeit in Form von Gauß-Krüger-Koordinaten erfaßt oder in das (3.) Gauß-KrügerSystem projiziert (vgl. Kap. 2.2).

Da im Nordwesten Niedersachsens ein Meer - die Nordsee - liegt, das nach Westen und Südwesten in den Atlantik übergeht, ist anzunehmen, daß mit größerem Rechtswert bzw. kleinerem Hochwert - weiter östlich bzw. südlich - der maritime Einfluß auf das Klima abnimmt, bzw. das Ortsklima kontinentaleren Charakter zeigt. Bezüglich der Depositionen liegt in diesen Richtungen eine immer größere Landfläche zwischen dem betrachteten Punkt und dem Meer. Bei meeresbürtigen 
Elementen sollte dies zu einer Verminderung, bei allen anderen Elementen zu einer Erhöhung der Depositionen führen.

Der Hochwert beschreibt seinerseits allerdings nicht nur den Abstand zur Küste und damit die Kontinentalität sondern auch - noch direkter - den Breitengrad. Diese beiden Eigenschaften können beispielsweise im Bezug auf die Lufttemperatur durchaus gegenläufig sein. Regionen in nördlicheren Breiten lassen grundsätzlich geringere durchschnittliche Lufttemperaturen erwarten. Im Falle Niedersachsens liegen diese aber auch näher am Meer und damit vor allem in Herbst und im Winter in dessen mäßigendem, erwärmendem Einfluß (Golfstrom). Als Maß für die Kontinentalität scheint somit der Rechtswert besser geeignet $\mathrm{zu}$ sein als der Hochwert.

\subsubsection{Abstand zur Küste}

Es liegt nahe, die kürzeste Entfernung der Meßstationen und Zielpunkte zur Küste als weiteren Index für die Kontinentalität zu definieren. Dieser kürzeste Abstand zur Küste wurde mit Hilfe des GIS ARC/Info ermittelt und diente als dritte lageabhängige Variable.

Eine vierte Variable, für deren Berechnung der Abstand zur Küste in Richtung Nordwest berechnet wurde, konnte bei keiner Zielvariablen einen signifikanten Beitrag zu einem Regionalisierungsmodell leisten. Der hohe Modellierungs- und Rechenaufwand wurde so nicht gerechtfertigt. Eine nähere Darstellung dieser vierten Variablen unterbleibt daher.

\subsection{Höhenabhängige Variablen}

\subsubsection{Geländehöhe}

Die Geländehöhe, die Höhe über NN, gilt als ein entscheidender Faktor zur Variabilität der Temperatur (vgl. z. B. Böhner 1996, Felbermeier 1994 oder Vent- 
Schmidt 1985). Je höher ein Ort über NN liegt, desto kälter sind die durchschnittlichen Temperaturen. Positiv korreliert ist die Geländehöhe zudem mit dem Niederschlag (ebenda) und mit Depositionen (vgl. z. B. ToBIAS et al., 1989 oder LENZ et al., 1996).

Die in dieser Arbeit verwendeten Werte für die Geländehöhe und aller aus ihr abgeleiteten Variablen (s.u.) wurden grundsätzlich mit Hilfe des GIS ARC/Info und eines Digitalen Höhenmodells mit Rasterweite 50 m (DGM50) ermittelt.

\subsubsection{Hangneigung und Hangrichtung}

Die Hangneigung an einem Rasterpunkt wird als die stärkste Abnahme der Geländehöhe je horizontale Entfernung definiert. Sie kann im Vergleich zu den umgebenden Punkten ermittelt und in Prozent der Entfernung oder Grad ausgedrückt werden. Eine Hangneigung von $100 \%$ entspricht einer solchen von $45^{\circ}$, auf k Meter horizontale Entfernung nimmt die Geländehöhe um k Meter ab. In dieser Arbeit wurde die Hangneigung in Prozent berechnet und zur Regionalisierung verwendet.

Diejenige Richtung, in welcher die Hangneigung gemessen wird, heißt Hangrichtung, $\alpha$. Ihre Einheit ist Grad $\left[^{\circ}\right] .0^{\circ}$ und $360^{\circ}$ entsprechen einer Ausrichtung nach Norden, $90^{\circ}$ nach Osten, $180^{\circ}$ nach Süden und $270^{\circ}$ nach Westen.

Die Hangrichtung als solche kann nicht als metrische Variable in einem linearen Regressionsmodell der Lufttemperatur verwendet werden. Wird am Osthang eines Berges (Hangrichtung: $90^{\circ}$ ) eine durchschnittliche tägliche Lufttemperatur von $8,0^{\circ} \mathrm{C}$ gemessen, und an der Südseite (Hangrichtung: $180^{\circ}=90^{\circ}+90^{\circ}$ ) eine solche von $8,2^{\circ} \mathrm{C}$, so wird für einen Westhang (Hangrichtung: $270^{\circ}=90^{\circ}+2 * 90^{\circ}$ ) keine Lufttemperatur von $8,4^{\circ} \mathrm{C}$ erwartet, was einem linearen Zusammenhang entspräche. Daher wurden zwei Transformationen der Hangrichtung durchgeführt. Als Hangneigung nach Süden, wurde die Abweichung der Hangrichtung zur Hangrichtung Nord definiert:

$$
\alpha_{s}=\left\{\begin{array}{r}
\alpha ; \alpha \leq 180^{\circ} \\
360^{\circ}-\alpha ; \alpha>180^{\circ}
\end{array}\right.
$$

Analog wurde als Hangrichtung nach Westen die Abweichung der Hangrichtung zur Hangrichtung Ost definiert: 


$$
\alpha_{\mathrm{w}}=\left\{\begin{array}{c}
90^{\circ}-\alpha ; \alpha \leq 90^{\circ} \\
\alpha-90^{\circ} ; 90^{\circ}<\alpha \leq 270^{\circ} \\
450^{\circ}-\alpha ; \alpha>270^{\circ}
\end{array}\right.
$$

So entstehen zwei neue metrische Variablen, die für die Varianzaufklärung unterschiedlicher Zielvariablen sinnvoll verwendet werden können.

Die Hangneigung nach Westen soll der Tatsache Rechnung tragen, daß westliche Winde im Testgebiet häufiger sind als solche aus anderen Windrichtungen (vgl. HÄCKEL, 1990, S. 288 f). Die Hangrichtung wurde auch von ToBIAS et al. (1989) und von LENZ et al. (1996) als einer von mehreren Faktoren beschrieben, welche Einfluß auf die Deposition atmogener Einträge haben. Auch für die Niederschlagssumme ist eine positive Korrelation anzunehmen. Für längere Hänge mit Westexposition könnten Steigungsregen $\mathrm{zu}$ einer Erhöhung der Niederschläge führen (vgl. Kap. 3.3.4).

\subsubsection{Lee-Indices}

Häckel (1990) beschreibt zwei unterschiedliche (Lee-) Effekte auf den Niederschlag, die durch das Überstreichen von Windmassen über Erhebungen der Erdoberfläche an der windabgewandten Hangseite verursacht werden können. Zudem soll hier noch auf die Erwärmung der Lufttemperatur durch adiabatische Effekte eingegangen werden, auf den Föhn.

Die Höhe des Niederschlages kann durch die Windverhältnisse extrem beeinflußt werden. Neben der Beeinträchtigung der Meßgenauigkeit durch den Wind (vgl. KUMM, 1983), hat er auch Einfluß auf die tatsächliche Höhe des Niederschlags. Als Lee-Effekt wird das Phänomen beschrieben, daß im Windschatten einer Geländeerhebung weniger Regen fällt als an anderen Geländestellen. Dies resultiert aus der Tatsache bzw. Möglichkeit, daß anströmende Luftmassen bereits einen Teil des enthaltenen Wassers an vorgelagerten Geländeerhebungen beim Überströmen verlieren. Die leewärts wieder abfallenden Luftmassen gleiten unter adiabatischer Erwärmung wieder ins Tal - Temperatureffekt, Föhn - und die relative Luftfeuchtigkeit liegt unter derjenigen von Luftpaketen, die sich vor der Geländeerhebung befinden oder sich über ebenes Gelände fortbewegen. Wolken lösen sich auf. Dies soll durch die Variable Lee-Effekt beschrieben werden. 
Im Gegensatz zu solchen den Niederschlag abschwächenden Effekten wurde aber vor allem bei hohen Windgeschwindigkeiten auch ein den Niederschlag verstärkender Effekt durch sogenannte Leewirbel beobachtet. Diese sollen durch eine zweite räumliche Variable beschrieben werden. Während der abschwächende LeeEffekt vor allem auf längere Distanzen und vor allem hinter größeren Geländeerhebungen wirksam ist - Häckel (1990, S. 291) nennt einige hundert Meter als hinreichend - ist der verstärkende Effekt durch Leewirbel bereits hinter kleineren Geländeerhebungen oder auch hinter Häusern und Bäumen zu beobachten. Dabei ist er auf kürzere Distanzen beschränkt.

Die Modellierung der beiden unterschiedlichen Effekte orientiert sich am Vergleich der Geländehöhe eines jeden Rasterpunktes $\mathrm{C} 0$ mit den Rasterwerten der Geländehöhe in westlich vom Zielpunkt C0 definierten Kreisausschnitten. Diese Kreisausschnitte unterscheiden sich durch unterschiedliche Radien und Breiten. Während für den weiträumig wirksamen Lee-Effekt zwei Variablen mit unterschiedlichen Radien $(25 \mathrm{~km}$ und $50 \mathrm{~km}$; Variablen: Lee-Effekt 25 und LeeEffekt 50) bei einer Breite von $90^{\circ}$ modelliert wurden, wurden für die Variable Leewirbel vier Radien (200 m, 500 m, $1 \mathrm{~km}$ und $2 \mathrm{~km}$ ) mit einer Breite von $60^{\circ}$ herangezogen. Grund für die geringere Breite des Kreisausschnittes bei der Berechnung der Variable Leewirbel war die geringere Rechenzeit $\mathrm{zu}$ ihrer Berechnung. Es wurden keine weiteren Radien oder Breiten der Kreisausschnitte getestet. Eine weitere explorative Analyse der Daten in Hinsicht auf eine optimale Beschreibung der Lee-Effekte könnte wertvolle Erkenntnisse liefern. Allerdings besteht dabei auch die Gefahr, die Modellierung an Windverhältnisse anzupassen, wie sie nur für die stichprobenartigen Messungen des DWD gelten.

Die Variable Leewirbel wurde als die Summe der 4 Differenzen zwischen den Geländehöhenmaxima der 4 verwendeten Kreisausschnitte (maxHöhe $\mathrm{i}_{\mathrm{i}}$ ) und der Geländehöhe des Zielrasterpunktes C0 (Höhe $(\mathrm{C} 0)$ definiert:

$$
\text { Leewirbel }(\mathrm{C} 0)=\sum_{\mathrm{i}=1}^{4}\left(\text { maxHöhe }_{\mathrm{i}}-\operatorname{Höhe}(\mathrm{C} 0)\right)
$$

Bei der Beschreibung des Lee-Effektes wurde neben dem Geländehöhenmaximum (maxHöhe) der Umgebungen bzw. Kreisausschnitte mit Radius n (25 km bzw. $50 \mathrm{~km}$ ) deren mittlere Geländehöhe (meanHöhe) berücksichtigt:

$$
\text { Lee }- \text { Effekt } \mathrm{n}(\mathrm{C} 0)=\text { maxHöhe } \mathrm{n}+\text { meanHöhe } \mathrm{n}-2 \bullet \operatorname{Höhe}(\mathrm{C} 0)
$$


Zusätzlich wurde eine Differenzbildung ausschließlich zwischen dem Maximum der Umgebung und der Höhe des Zielrasterpunktes zur Definition einer weiteren Variablen durchgeführt. Diese wurde aber später nicht in die Regionalisierungsmodelle integriert, da die Variablen Lee-Effekt 50 und Lee-Effekt 25 ein höheres Erklärungspotential zeigten.

Eine Besonderheit der Variablen Lee-Effekt 50 (25) liegt in der Ausdehnung des zu ihrer Berechnung nötigen Digitalen Höhenmodells (DHM). Da die Variable über den Vergleich mit Höhenwerten in einem Kreisausschnitt von $225^{\circ}$ bis $315^{\circ}$ (SW bis NW) mit einem Radius von $50 \mathrm{~km}(25 \mathrm{~km})$ definiert wird (Kap. 3.3.3), ist es vorteilhaft, zu ihrer Berechnung ein DHM zu verwenden, das um mindestens $50 \mathrm{~km}$ $(25 \mathrm{~km})$ über das Zielgebiet hinausragt. Zur Bestimmung der Stationswerte sollten zumindest die Umgebungen der Stationen durch das DHM abgedeckt sein. Im Falle des verwendeten DHM (DGM50 des Landesvermessungsamtes Niedersachsen) konnten diese Bedingungen nicht erfüllt werden. Da jedoch westlich des niedersächsischen Berg- und Tieflandes keine signifikant zu dem niedersächsischen Gebiet verschiedenen orographischen Verhältnisse herrschen - das Weserbergland hört nicht westlich der Landesgrenze auf bergig zu sein -, wurde eine Berechnung der Variable gewählt, die Lücken im DHM ignoriert. Die ARC/Info-Funktionen $<$ focalmean> und < focalmax > bieten hierzu die Option „,data“ an. Sind nur Teile der Umgebung mit Werten des Ausgangsrasters (hier: Geländehöhe) belegt, so werden nur diese zur Berechnung des Funktionswertes herangezogen. Alternativ besteht die Möglichkeit, nur für Zellen, deren komplette Umgebung durch das DHM abgedeckt wird, Funktionswerte zu berechnen. Diese Option wurde hier bewußt nicht gewählt. Ein Band von $50 \mathrm{~km}(25 \mathrm{~km})$ Ausdehnung am Westrand des Untersuchungsgebietes hätte sonst aus den Untersuchungen ausgeklammert werden müssen.

\subsubsection{Luv-Index}

Analog zu den Lee-Indices wurde auch eine Variable berechnet, die den oben bereits angedeuteten, den Niederschlag verstärkenden Staueffekt an Luv-Hängen beschreiben sollte. An Hindernissen wie Gebirgen aufgleitende Luftmassen kühlen sich ab, was zu einer Erhöhung der relativen Luftfeuchtigkeit führt. Der Taupunkt wird unterschritten, Wasser kondensiert, Wolken bilden sich und es fällt Niederschlag. 
Entsprechend der Definition der Variable Leewirbel wurden auch hier vier Kreisausschnitte mit dem Radius 200 m, 500 m, 1000 m und 2000 m - hier allerdings nach Osten gerichtet (von $60^{\circ}$ bis $120^{\circ}$ ) - als Umgebung für jeden Zielrasterpunkt gewählt. Die Differenzen zwischen den Umgebungsmaxima der Geländehöhe (maxHöhe $\mathrm{i}_{\mathrm{i}}$ ) und der Geländehöhe am Zielrasterpunkt (Höhe $\left.\mathrm{C}_{(\mathrm{C})}\right)$ wurden summiert:

$$
\text { Luvsumme }(\mathrm{C} 0)=\sum_{\mathrm{i}=1}^{4}\left(\text { maxHöhe }_{\mathrm{i}}-\text { Höhe }(\mathrm{C} 0)\right)
$$

Von dieser Variable wird eine positive Korrelation mit dem Niederschlag erwartet, da stärkere Erhöhungen im Osten zu häufigen Staulagen und somit zu mehr Niederschlag führen sollten. Auch weitere Variablen, die den nach Osten definierten Variablen Lee-Effekt 25 und Lee-Effekt 50 entsprechen, wurden definiert und für die Niederschlagmeßstationen ermittelt, sie konnten aber keinen Beitrag zur Bildung von Regionalisierungsmodellen leisten.

\subsubsection{Relative Exponiertheit}

Die Variable Relative Exponiertheit (kurz: EXP), im Rahmen dieser Arbeit auch schlicht mit Exponiertheit bezeichnet, wurde von SCHULZ (in Vorb.) entwickelt, um $\mathrm{zu}$ beschreiben, ob ein Ort durch das ihn umgebende Gelände vor Umwelteinwirkungen geschützt oder ihnen relativ ungeschützt ausgesetzt ist (z. B. Wind). Die Relative Exponiertheit beschreibt auch die potentielle nächtliche Ausstrahlung und somit Abkühlung. BLENNOw \& PERSSON (1998) verwendeten zu diesem Zweck einen sogenannten ,sky view factor", für den von DOZIER \& FREW (1990) ein Weg zur Berechnung vorgeschlagen wird. Da auf vorhandene Algorithmen eines weit verbreiteten GIS zurückgegriffen werden sollte, fand dieser Ansatz keine Verwendung.

Die Relative Exponiertheit beschreibt die Lage eines Ortes über die Zahl von Richtungen mit $10^{\circ}$ Azimut-Abstand (36 Richtungen), aus denen ein mit $8^{\circ}$ Neigung einfallender Strahl den Ort erreichen kann, ohne durch das umliegende Gelände aufgehalten zu werden. Dieser Ansatz ist nicht direkt vergleichbar mit einem Faktor, der den Anteil des offenen Himmels beschreibt, da es für den Wert der Relativen Exponiertheit keinen Unterschied macht, ob ein hochaufragendes Gebirge einen Strahl abhält oder ein kleiner Hügel, der den Strahl mit $8^{\circ}$ Neigung nur knapp 
abdeckt, einen Strahl mit $9^{\circ}$ jedoch passieren ließe. Allerdings wird ein benachbartes Gebirge die Strahlen aus vielen Richtungen aufhalten, eine kleine Geländeerhebung in gleicher Entfernung nur Strahlen aus wenigen Richtungen, weshalb die Ähnlichkeit beider Ansätze größer sein dürfte als die unterschiedliche Definition es zunächst erwarten läßt. Die Relative Exponiertheit ist durch die ARC/Info-Funktion $<$ hillshade> schnell und einfach zu berechnen. Eine aufwendige Programmierung ist nicht erforderlich.

Der Versuch mit unterschiedlichen Neigungswinkeln der Strahlen ergab die gleichmäßigste Differenzierung der Exponiertheit im Gebiet des Forstamtes Seesen/Harz bei einem Winkel von $8^{\circ}$. In der Norddeutschen Tiefebene war jedoch die Streuung dieser Variable auch bei nur $8^{\circ}$ sehr gering. Für fast alle Zellen wurde der Wert 36 ermittelt, da keine Beschattung/Beschirmung stattfand. Daher wurde ein zusätzlicher Faktor, die relative Höhe, integriert und damit eine neue Variable, die Exponiertheit zum Umgebungsminimum (kurz: expmin), definiert.

Die relative Höhe wurde für eine Umgebung ermittelt, die als Kreis mit dem Radius $500 \mathrm{~m}$ definiert wurde. Die Differenz zwischen der Geländehöhe des Zielrasterpunktes (Höhe $(\mathrm{C} 0)$ ) und dem Minimum der Umgebung (minHöhe) wurde zum Wertebereich der Höhe in dieser Umgebung (rangeHöhe) ins Verhältnis gesetzt. Die komplette Formel für die Berechnung von expmin weist zudem im Nenner noch die Addition von 0,01 auf um Nenner des Wertes $0 \mathrm{zu}$ vermeiden, die in absolut ebenem Gelände vorkommen:

$$
\operatorname{expmin}(\mathrm{C} 0)=\exp \bullet \frac{\text { Höhe }(\mathrm{C} 0)-\text { minHöhe }}{\text { rangeHöhe }+0.01}
$$

Die so definierte Variable expmin zeigt auch in ebenem Gelände ausreichende Variabilität, um zur Varianzaufklärung von Zielvariablen beitragen zu können.

Für die Temperaturmeßstationen ist der einfache Korrelationskoeffizient zwischen expmin und der in ihr enthaltenen relativen Höhe nahezu gleich 1 (Niedersachsen: 0.99535; Bergland: 0.98556; Tiefland: 0.99998). Expmin wurde in dieser Arbeit favorisiert, da von dieser Variable auf das Gesamtgebiet und besonders auf das Bergland bezogen ein höheres Erklärungspotential erwartet wurde. 


\subsubsection{Kaltluftabfluß}

Diese von SchUlZ (in Vorb.) eingeführte Variable ähnelt der von LAUGHLIN \& KALMA (1990) zur Regionalisierung von Frostgefährdung verwendeten räumlichen Variablen Flowaccumulation. Ein Unterschied ist dabei nicht in der Konzeption bzw. Definition sondern vielmehr in der Ausdehnung der einbezogenen Fläche zu sehen. Schulz (in Vorb.) definierte die Variable als die Anzahl derjenigen Zellen, die in die beobachtete Zelle C0 entwässern, was sich mit einem geeigneten digitalen Höhenmodell und dem GIS ARC/Info relativ problemlos ermitteln läßt. Bei der Berechnung werden grundsätzlich keine Grenzen nach oben für den resultierenden Wert angegeben. Da jedoch eine Fläche über 500 ha für die Beschreibung des Risikos von Kaltluftabfluß nicht sinnvoll erscheint, wurden die Werte bei dem entsprechenden Wert von 2000 Zellen gekappt.

Da vor allem in Tälern ein relativ abrupter Übergang zwischen sehr hohen und weniger hohen Werten Folge der einfachen Berechnung mit der Funktion $<$ flowaccumulation> ist, wurde von ScHULZ (in Vorb.) zweimal der Mittelwert aller benachbarten Zellen und der Zielzelle $\mathrm{C} 0$ für letztere ermittelt (Funktion <focalmean>), was eine gewünschte Glättung der Werte im digitalen Raster zur Folge hatte (Variablenname: Kaltluftabfluß).

Da weiterhin sehr große Werte vielen kleinen Werten gegenüberstanden (extrem rechtsschiefe Verteilung der Werte) wurde die Variable transformiert. Durch die Berechnung der Quadratwurzel von Kaltluftabflu $\beta$ wurde die Dimension von einer flächigen in eine eindimensionale Größe transformiert, deren Verteilung geeigneter erschien, das Risiko von Kaltluftabfluß zu beschreiben (Variablenname: QWKaltluftabfluß).

Während die Variable Kaltluftabfluß wegen ihrer besseren räumlichen Interpretierbarkeit zur Beschreibung der Datenkollektive verwendet wurde (Kap. 4), wurde die Variable QWKaltluftabflu $\beta$ bei der Regionalisierung der Lufttemperatur als potentieller Regressor eingesetzt. 


\subsubsection{Insolation}

Um die potentielle Einstrahlung auf jeden Rasterpunkt eines digitalen Höhenmodells (DHM) quantifizieren zu können, wurde von ScHUlz (in Vorb.) für jeden Monat der Sonnenstand zu jeder vollen Stunde ermittelt und mit der Hillshade-Funktion des GIS ARC/Info simuliert (Kap. 3.2.4). So entstanden die Variablen jandow bis dezdow (Anzahl der eingehenden Strahlen in der Mitte des Monats von Januar bis Dezember). Zusätzlich zum puren Erreichen der Rasterpunkte durch die Strahlen wurden weitere Variablen hergeleitet, die einerseits die Abschwächung der Einstrahlung durch unterschiedlich lange Bahnen der Strahlen durch die Erdatmosphäre (gekennzeichnet durch -att am Ende des Variablenkürzels) und andererseits den Winkel, in dem die Strahlen auf die Erdoberfläche treffen, modellieren (-all statt -dow im Variablenkürzel). Alle diese Variablen konnten in den anschließenden Regressionsanalysen nicht signifikant zur Aufklärung der Varianzen der Zielvariablen beitragen. Die im Rahmen dieser Arbeit ausschließlich zur Repräsentanzanalyse verwendete Variable Insolation im Januar (Variablenkürzel: janallatt) ist ein dimensionsloser Index. Die Einheit dieser Variable wird daher mit ,[] ]“ angegeben.

\subsection{Variablen aus Kartierungen}

\subsubsection{Landnutzungsklassen}

Landsat TM-Satelliten-Aufnahmen dienten als Grundlage für eine digitale Klassifizierung der Landnutzungstypen in Niedersachsen. Das Umweltministerium des Landes Niedersachsen stellte die Ergebnisse dieser Klassifizierung dankenswerterweise im Internet zur Verfügung (unter ,http://www.mu.niedersachsen.de"). Die in Tabelle 2 aufgeführten Landnutzungsklassen wurden 4 Gruppen zugeordnet: 
Tabelle 2: Landnutzungsklassen der Klassifizierung des Niedersächsischen Umweltministeriums und deren Einordnung in Gruppen zur weiteren Analyse

\begin{tabular}{llll}
\hline Nummer & \multicolumn{1}{c}{ Landnutzung } & Zuordnung/Gruppe & Variablenname \\
\hline 0 & Außerhalb Niedersachsens & & \\
11 & Ackerland & Landwirtschaft & Acker \\
12 & Gewässer & Gewässer & Gewässer \\
13 & Grünland & Landwirtschaft & Acker \\
14 & Laubwald & Wald & Wald \\
15 & Dorf / Vorstadt & Stadt & Bebauung \\
16 & Park / Friedhof / Obstanlagen & Wald & Wald \\
17 & Mischwald & Wald & Wald \\
18 & Moor & Gewässer & Gewässer \\
19 & Stadt & Stadt & Bebauung \\
20 & versiegelte Flächen & Stadt & Bebauung \\
21 & Ödland & Landwirtschaft & Acker \\
22 & Nadelwald & Wald & Wald \\
23 & n. klassifiziert & & \\
24 & Schlagkulturen, Aufforstungen, & Wald & Wald \\
& aufgelichteter Wald & & \\
25 & Watt & Gewässer & Gewässer \\
26 & Heide & Landwirtschaft & Acker \\
27 & Sand / Dünen & Landwirtschaft & Acker \\
28 & Heide / Moor n. trennbar & Landwirtschaft & Acker \\
\hline
\end{tabular}

Aus dem so vereinfachten Gruppenraster wurde der Anteil der vier Gruppen an der Fläche unterschiedlich großer Umgebungen in Prozent berechnet, wodurch metrische Variablen generiert wurden (vgl. Tabelle 3). Die Umgebungen wurden als Kreise mit unterschiedlichem Radius definiert. Um den Rechenaufwand bei großen Radien in vertretbarem Rahmen zu halten, wurden die verwendeten Rasterweiten der Ergebnisraster größer gewählt als die Rasterweite des Ausgangsgrids (30 m). Diese Vorgehensweise entspricht einer Stichprobennahme und führte dazu, daß unterschiedlich viele Punkte bei der Anteilsberechnung berïcksichtigt wurden.

Tabelle 3: Radius and entsprechende Rasterweiten für die Berechnung der Landnutzungsvariablen

\begin{tabular}{rccc}
\hline $\begin{array}{c}\text { Radius } \\
{[\mathbf{m}]}\end{array}$ & $\begin{array}{c}\text { Rasterweite } \\
{[\mathbf{m}]}\end{array}$ & $\begin{array}{c}\text { einbezogene } \\
\text { Rasterpunkte }\end{array}$ & $\begin{array}{c}\text { Kürzel } \\
(\mathbf{z .} \text { B. Wald5h1h) }\end{array}$ \\
\hline 500 & 100 & 78 & $x 5 \mathrm{~h} 1 \mathrm{~h}$ \\
1000 & 100 & 314 & $x 1 \mathrm{t} 1 \mathrm{~h}$ \\
2000 & 200 & 314 & $x 2 \mathrm{t} 2 \mathrm{~h}$ \\
5000 & 500 & 314 & $x 5 \mathrm{t} 5 \mathrm{~h}$ \\
10000 & 500 & 1256 & $x 10 \mathrm{t} 5 \mathrm{~h}$ \\
\hline
\end{tabular}

Die Anzahl der einbezogenen Rasterpunkte wurde durch den Quotienten aus der Flächengröße der Umgebung und der Fläche einer Zelle des Ergebnisrasters geschätzt. Selbst im Fall der kleinsten Umgebung wurden noch 78 Stichprobenpunkte berücksichtigt (s. Tabelle 3). Da schon im Laufe der ersten 
Analysen Radii von 1 bis $5 \mathrm{~km}$ das höchste Erklärungspotential aufwiesen, wurden zur Modellbildung schließlich bei teilweise geringfügig niedrigerer Varianzaufklärung nur die Variablen mit einem Radius von 2 km verwendet.

\subsubsection{Fachdaten}

Zur Präzisierung von Landnutzungsinformationen können auch fachspezifische Informationslagen hinzugezogen werden. Insbesondere Waldmeßstationen können ohne solche Informationen nicht zufriedenstellend bezüglich ihrer Meßsituation beschrieben werden. Forsteinrichtungsdaten waren zur Zeit der Erstellung der vorhandenen Datenbasis noch nicht flächendeckend digital vorhanden. Eine Weiterentwicklung in diese Richtung ist aus Sicht der Regionalisierung wünschenswert und wäre sicher sehr vorteilhaft. Auf lange Sicht werden digitale Grundinformationen helfen, Zeit und Kosten in nicht unerheblichem Maße zu sparen (z. B. KONITZER, in Vorb.).

\subsubsection{Forsteinrichtung}

Durch eine schriftliche Anfrage bei den zuständigen Forstämtern ließ sich nur ein sehr lückenhaftes Bild der Bestandesverhältnisse zur Zeit der Beprobung von Waldstationen ermitteln. In den meisten Fällen konnte so nur auf Informationen aus der Literatur zurückgegriffen werden. Diese beschränken sich nur allzu oft auf wenige Angaben, die einer Bestandesbeschreibung kaum genügen können. Auf diesem Wege konnten für nahezu alle Waldstationen des Depositionsmeßnetzes die Hauptbaumart (kategoriell) und deren Durchschnittsalter (metrisch) ermittelt werden. Andere Variablen wie Bestandeshöhe und Bestockungsgrad konnten nur für wenige Bestände ermittelt werden. Im Rahmen der durchgeführten statistischen Analysen wurde daher lediglich bei der Depositionsregionalisierung auf die Hauptbaumart an den verwendeten Waldmeßstationen zurückgegriffen. Wegen des Mangels an flächendeckender digitaler Informationen bezüglich anderer Bestandesmerkmale wäre eine GIS-gestützte Umsetzung von Regionalisierungsfunktionen, die weitere Variablen der Bestandesbeschreibung verwenden, ohnedies nicht möglich. 
Die kategorielle Variable Hauptbaumart wurde für die Analyse des Depositionsdatenkollektives in Dummy-Variablen transformiert. Es wurde unterschieden zwischen Waldmeßstationen und Freilandstationen (Dummy-Variable Wald), zwischen Baumartengruppen und schließlich bezüglich der Zugehörigkeit zu einer bestimmten Baumart (z. B. Nadel, Laub, Fichte, Buche). Einen Überblick bietet Tabelle 4. Positive Beantwortung der jeweiligen Frage führt zur Belegung der Dummy-Variable mit dem Wert 1, andernfalls wird der Wert 0 gewählt. Je nach Depositionsverhalten und nach der Betrachtung des gesamten Depositionsdatenkollektivs oder einzelner Unterkollektive (Wald- oder Freilandkollektiv) können für einzelne Dummy-Variablen positive oder negative Korrelationskoeffizienten in einem Regressionsmodell plausibel sein. So sollte für Buche bei der Analyse des Gesamtkollektivs ein positiver Regressionskoeffizient, bei der Analyse des Waldkollektivs aber ein negativer Regressionskoeffizient erwartet werden.

Tabelle 4: Aus der kategoriellen Variable Hauptbaumart abgeleitete DummyVariablen

\begin{tabular}{cl}
\hline Variablenname & \multicolumn{1}{c}{ Frage $(\mathbf{j a} \Rightarrow \mathbf{1}$, nein $\Rightarrow \mathbf{0})$} \\
\hline Wald & Ist die Station eine Waldmeßstation? \\
Nadel & Ist die Hauptbaumart eine Nadelbaumart ? \\
Laub & Ist die Hauptbaumart eine Laubbaumart? \\
BaumartX & Ist die Hauptbaumart die Baumart X? \\
\hline
\end{tabular}

\subsubsection{Interzeptionsindex}

Ausgehend von der Kenntnis über Hauptbaumart und deren Durchschnittsalter wurde ein Index für die Interzeption generiert. Als Hilfsmittel dienten hier die Normalertragstafeln für Buche (nach ScHOBER 1967) und Fichte (nach WIEDEMANN 1936/42, s. Schober, 1987). In ihnen wurde eine Größe gesucht, die steigende Werte bis in das Jugendalter und anschließend fallende Werte zeigt. Diese soll näherungsweise dem Interzeptionsvermögen der Bestände entsprechen, das wesentlich durch ihre innere Oberfläche und die Rauhigkeit der Bestände bestimmt wird. Beides sollte im Jugendalter eines Bestandes durch hohe Blattmassen und die Erhöhung der Rauhigkeit durch relativ häufige Durchforstungen gegeben sein (vgl. FRÄNZLE et al., 1989)

Die hier verwendete Größe aus der Ertragstafel ist die Derbholzformzahl $\mathrm{f}_{\mathrm{d}}$. Sie erfüllt die genannten Bedingungen - Anstieg der Werte im Jugendalter und dann Verringerung auf ein relativ konstantes Niveau über dem des Dickungsalters - für 
einen Interzeptionsindex. Sie muß nicht wie ein mathematisches Modell (z. B. Polynomialmodell über dem Alter) an Meßdaten angepaßt werden, sie stellt einen an umfangreichem Stichprobenmaterial gefundenen Zusammenhang dar.

Da die Derbholzformzahl erst ab der zweiten Nachkommastelle variiert, wurde zunächst 90 von dem hundertfachen Wert von $\mathrm{f}_{\mathrm{d}}$ subtrahiert. Der resultierende Wert, fortan mit $f_{d 90}$ bezeichnet, war Grundlage für die Entwicklung eines Interzeptionsindexes. Die Werte von $\mathrm{f}_{\mathrm{d} 90}$ zeigen für Fichte der zweiten Ertragsklasse ein Maximum im Bereich zwischen 50 und 60 Jahren. Die Werte für alte Bestände schienen aber im Vergleich zu denen von Beständen mit einem Alter unter 40 Jahren zu hoch. Außerdem wurde für Buche der 2. Ertragsklasse kein solches Maximum gefunden. Daher wurde eine Modifikation dieser Größe vorgenommen: Der Interzeptionsindex, Intind 1 , wurde als Quotient aus $\mathrm{f}_{\mathrm{d} 90}$ und der Mittelhöhe, $\mathrm{h}_{\mathrm{m}}$, definiert:

$$
\text { Intind } 1=\frac{\mathrm{f}_{\mathrm{d}} * 100-90}{\mathrm{~h}_{\mathrm{m}}}
$$

Auch Modifikationen unter Verwendung der Quadratwurzel bzw. der vierten Wurzel aus der Mittelhöhe wurden definiert und für das Depositionsdatenkollektiv getestet (s. Kap. 5.2):

$$
\begin{aligned}
& \text { Intind } 2=\frac{\mathrm{f}_{\mathrm{d}} * 100-90}{\sqrt{\mathrm{h}_{\mathrm{m}}}} \\
& \text { Intind } 3=\frac{\mathrm{f}_{\mathrm{d}} * 100-90}{\sqrt[4]{\mathrm{h}_{\mathrm{m}}}}
\end{aligned}
$$

Die Werte der so definierten Indices und der originären Ertragstafelwerte sind in Anhang 1 für Fichte bzw. Nadelbaumarten und in Anhang 2 für Buche bzw. Laubbaumarten zusammengefaßt. Eine weitere Aufspaltung wurde im Rahmen dieser Arbeit nicht vorgenommen, da fast alle beprobten Nadelbaumbestände mit Fichte und die meisten Laubbaumbestände des Depositionsdatenkollektives mit Buche bestockt sind (Kap. 4.2).

Die Größenordnung der so definierten Indices wird in Abbildung 6 und in Abbildung 7 verdeutlicht. Während der absolute Wert der Indices keine Bedeutung 
für die Verwendung als Regressor besitzt, ist die Veränderung der Werte mit dem Alter für eine erfolgreiche Varianzaufklärung der Zielvariablen entscheidend.

Die hier vorgestellten Interzeptionsindices zeigten zwar durchaus signifikante Korrelationen mit Depositionsmessungen, fanden aber wegen des Vorhandenseins von Regressoren mit höherem Erklärungspotential in keinem Regressionsmodell Berücksichtigung.

Abbildung 6: Interzeptionsindices für Fichte bzw. Nadelbaumarten

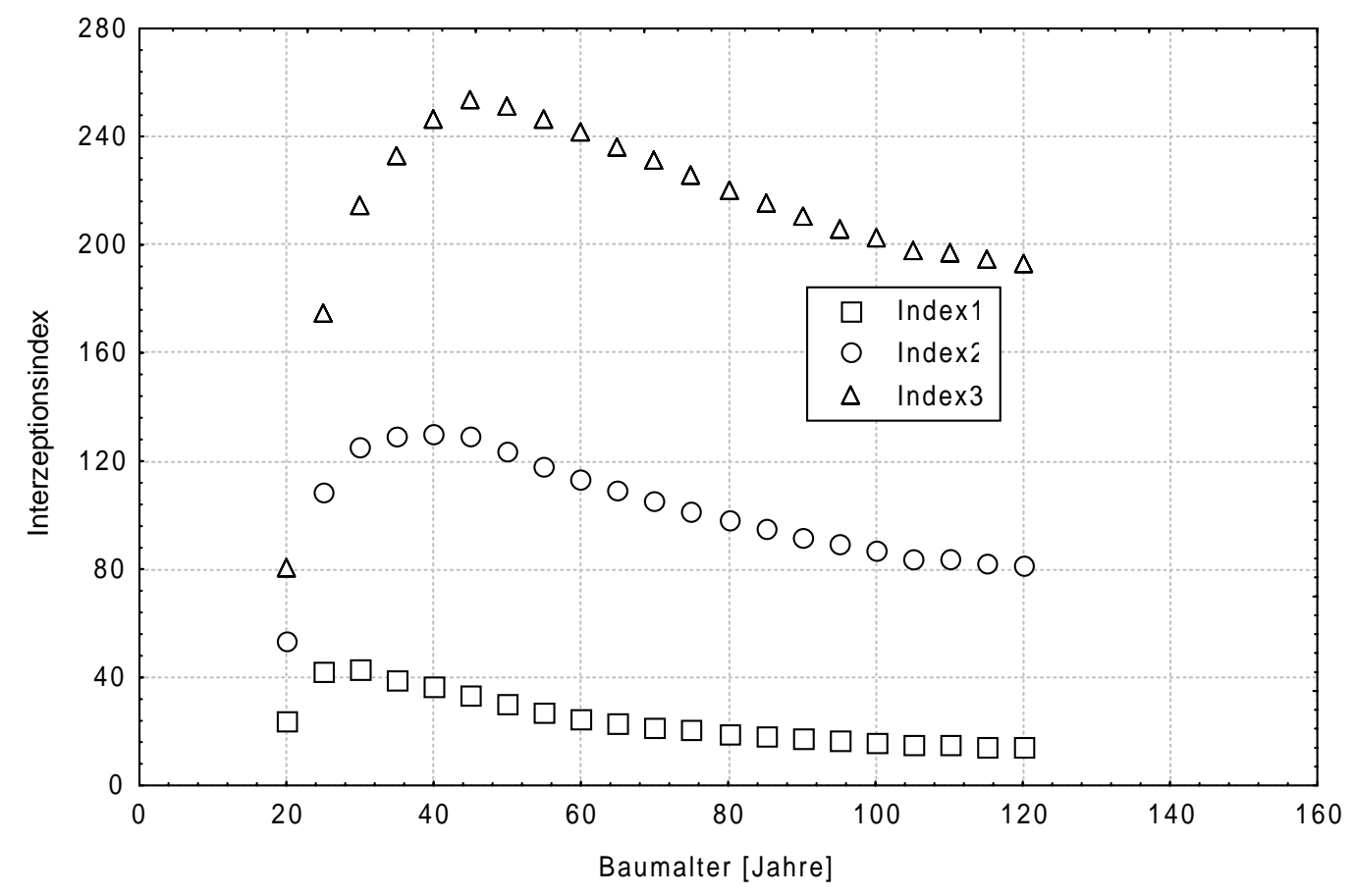

Abbildung 7: Interzeptionsindices für Buche bzw. Laubbaumarten

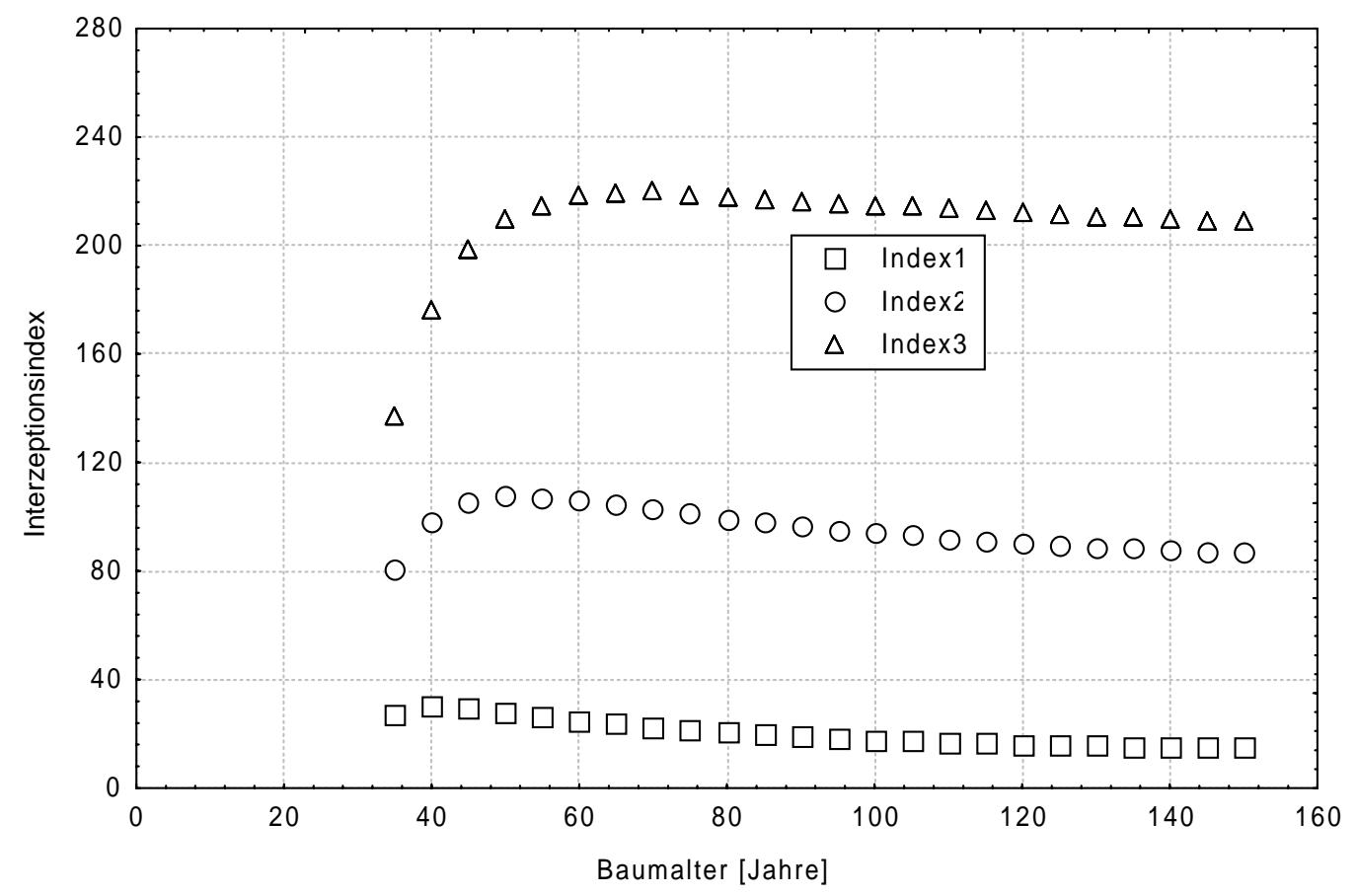




\section{Verwendete Daten}

Informationen über die räumliche Situation an den Meßstationen können genutzt werden, um die Repräsentanz des verwendeten Datenkollektivs für das Untersuchungsgebiet zu überprüfen. Diesem Aspekt wird ein großer Teil des vorliegenden Kapitels gewidmet. Er ist bei der Übertragung punkthaft gemessener Informationen auf die Fläche zusätzlich zu den Aspekten der Genauigkeit bei der Datengewinnung entscheidend für die Gültigkeit der gefundenen Regionalisierungsmodelle für das Zielgebiet.

Die in dieser Arbeit verwendeten Daten stammen aus unterschiedlichen Meßnetzen in Niedersachsen. Einen ersten Überblick über die verwendeten Datensätze gibt Tabelle 5:

Tabelle 5: Meßnetze und Quellen aus denen die in dieser Arbeit verwendeten Datensätze stammen

\begin{tabular}{|c|c|c|}
\hline & Meßnetz / Quelle & Zielgröße \\
\hline Klimadaten & Deutscher Wetterdienst (DWD) & $\begin{array}{l}\text { Niederschlagssumme, Lufttemperatur, } \\
\text { relative Luftfeuchte }\end{array}$ \\
\hline Klimadaten & Harzwasserwerke (HWW) & Niederschlagssumme \\
\hline Klimadaten & Institut f. Bioklimatologie & Lufttemperatur \\
\hline Depositionsdaten & $\begin{array}{l}\text { Niedersächsisches Landesamt für } \\
\text { Ökologie }\end{array}$ & $\begin{array}{l}\text { Bulk- Depositionen von } \mathrm{Na}, \mathrm{K}, \mathrm{Cl} \text {, SO4- } \\
\mathrm{S}, \mathrm{NO} 3-\mathrm{N} \text { und NH4-N }\end{array}$ \\
\hline Depositionsdaten & $\begin{array}{l}\text { Niedersächsisches Landesamt für } \\
\text { Ökologie, Abteilung Lufthygiene }\end{array}$ & $\begin{array}{l}\text { Bulk- Depositionen von } \mathrm{Na}, \mathrm{K}, \mathrm{Mg}, \mathrm{Fe} \\
\mathrm{Mn}, \mathrm{Al}, \mathrm{SO} 4-\mathrm{S}, \mathrm{Cl} \text { und NO3-N, }\end{array}$ \\
\hline Depositionsdaten & $\begin{array}{l}\text { Forschungszentrum } \\
\text { Waldökosysteme }\end{array}$ & $\begin{array}{l}\text { Bulk- Depositionen von Na, K, NH4-N, } \\
\mathrm{Mg}, \mathrm{Fe}, \mathrm{Mn}, \mathrm{Al}, \mathrm{SO} 4-\mathrm{S}, \mathrm{P}, \mathrm{Cl}, \mathrm{NO} 3-\mathrm{N}\end{array}$ \\
\hline
\end{tabular}

Die Datengewinnung durch Meßnetze kann als gerichtete stichprobenartige Erfassung zeitlich/räumlicher stochastischer Prozesse aufgefaßt werden. Hierbei wählt der Betreiber des Meßnetzes je nach Intention unterschiedliche Wege. Dieser Aspekt der zielorientierten Meßnetzplanung führt zu typischen Ergebnissen der Repräsentanzuntersuchungen, die hier u. a. dargestellt werden.

Die Beschreibung der Meßnetze soll jeweils eingeleitet werden mit einer Darstellung der räumlichen Verteilung bzw. der Lage der Stationen. Es folgt eine deskriptive Beschreibung der analysierten Meßwerte bzw. der zu Mittelwerten aggregierten Meßwerte. Desweiteren wurden die Verteilungen der in Kap. 3 erläuterten Variablen an den Meßstationen mit denjenigen entsprechender Raster für das Untersuchungsgebiet Niedersachsen verglichen. Dieser Vergleich orientiert sich grundsätzlich an 
einfachen deskriptiven Statistiken, den Lokalisationsmaßen arithmetischer Mittelwerte, Minimum und Maximum und dem Distributionsmaß Standardabweichung. Zudem wurden Häufigkeitsverteilungen der Stationsparameter und des gesamten Rasters für einige Variablen graphisch dargestellt, was die Beurteilung der Repräsentanz erleichtern soll (z. B. Abbildung 10, S. 59).

\subsection{Datenbasis Klima}

Im folgenden werden die den Daten zugrunde liegenden Meßmethoden und deren Fehler kurz erläutert. Weitergehende Informationen über den Aufbau und Eigenschaften von verwendeten Meßinstrumenten kann der Anleitung für die Beobachter der Klimahauptstationen des Deutschen Wetterdienstes (DWD, 1980) in Kombination mit meteorologischen Fachbüchern (z. B. HÄCKEL, 1990, S. 319ff) entnommen werden. Desweiteren sei auf die Seiten des Deutschen Wetterdienstes im Internet (http://www.dwd.de) verwiesen. Unter den Stichworten Klimainformationssystem bzw. Klimadatenbank können dort weitere Informationen zu Meßnetzen des DWD abgefragt werden.

Vom DWD wurden für die geplanten räumlichen Analysen langjährige (1961 bis 1990) monatliche und jährliche Mittelwerte zu den klimatischen Elementen Temperatur, Niederschlag und Relative Luftfeuchtigkeit zur Verfügung gestellt. Zusätzliche Niederschlagsdaten von Meßstationen der Harzwasserwerke (HWW), die in Ausstattung und Beprobung denen des DWD gleichen, wurden in die Datenbasis aufgenommen. Die Temperaturdatenbasis konnte durch die Integration eines Datenkollektivs des Institutes für Bioklimatologie erweitert werden. Deskriptive Statistiken zu den monatlichen und jährlichen Werten der behandelten Klimaelemente sind nach Datenkollektiven getrennt zu Beginn der entsprechenden Unterkapitel zusammengestellt (Kap. 4.1.2.1 bis 4.1.4.2).

Durch ihre freundliche Unterstützung und Betreuung bei Recherchen haben sich einige Mitarbeiter des DWD besonders um diese Arbeit verdient gemacht. Stellvertretend für alle anderen seien hier Frau Asmus (Geschäftsfeld Hydrologie) und Herr Liepelt (GF Meßnetzbetreuung) erwähnt. 
Zunächst soll auf Verortung und spezifische Probleme durch Verlegungen von Stationen während des Beobachtungszeitraums eingegangen werden (Kap. 4.1.1). Anschließend werden die Datenkollektive für durchschnittliche Lufttemperatur, durchschnittliche Niederschlagssumme und durchschnittliche relative Luftfeuchte dargestellt und auf ihre Repräsentanz für das Zielgebiet hin analysiert. Diese Reihenfolge der Klimaelemente wurde gewählt, da für den Temperaturdatensatz größere Repräsentanzmängel $\mathrm{zu}$ verzeichnen waren als für die Niederschlagmeßstationen. Das Datenkollektiv für die relative Luftfeuchte umfaßt bis auf eine Ausnahme die gleichen Meßstationen wie das Temperaturdatenkollektiv des DWD, weshalb eine Darstellung der Unterschiede dieser beiden Datenkollektive zuletzt erfolgt.

\subsubsection{Verortung der Stationen und Berücksichtigung von Meßortverlegungen}

Ein besonderes Problem bei den Datensätzen des DWD stellte sich durch die Möglichkeit von Verlegungen der Meßstationen während des Beobachtungszeitraums. Niederschlagmeßstationen und Klimahauptmeßstationen des DWD werden durch ehrenamtliche Mitarbeiter zumeist in der Nähe ihrer Wohnungen betrieben. Durch Beobachterwechsel oder Umzug des Beobachters kann es zu Verlegungen der Stationen kommen. Diese bewegen sich zumeist im Rahmen von unter hundert Metern bis zu wenigen Kilometern im niedersächsischen Tiefland. Nach Möglichkeit wird bei der Suche neuer Beobachter für eine bestehende Station darauf geachtet, daß die Verlegung zu einer Meßsituation führt, die der bisherigen ähnlich ist. Die Geländehöhe ist dabei eine relativ einfach zu ermittelnde und für die Ausprägung der Meßergebnisse bedeutende Größe.

Alle Vorgänge, die eine Meßstation betreffen, wie deren Kontrolle durch hauptamliche Mitarbeiter, Austausch oder Reparatur von Meßinstrumenten oder eben Beobachterwechsel und Verlegungen sind in den Unterlagen des DWD dokumentiert. Zumindest für die aktuelle Position der Stationen werden GaußKrüger-Koordinaten geführt. Im Falle der Wetter- und der Klimahauptstationen wurde die Lage der Stationen auch für frühere Standorte in Form von Gauß-KrügerKoordinaten und/oder geographischen Koordinaten dokumentiert. 
An einem Beispiel soll zunächst verdeutlicht werden, wie sich Verlegungen in der Realität darstellten. Hierzu wurde die Verlegungsgeschichte der Station „Sieber“ gewählt (s. Abbildung 8), da diese sehr deutliche wenn auch nicht die extremsten Verlegungseffekte zeigt. Zudem wurde sie im Untersuchungszeitraum dreimal verlegt. $\mathrm{Zu}$ den Häufigkeiten der Meßortverlegungen sind die Angaben in Tabelle 6 $\mathrm{zu}$ beachten. Es wurden keine Meßorte berücksichtigt, an denen eine Meßstation kürzer als ein halbes Jahr verortet war.

Tabelle 6: Anzahl der Meßorte (Stationsverlegungen+1) von 35 Niederschlagmeßstationen im Harz

\begin{tabular}{cc}
\hline Anzahl Meßorte & Häufigkeit \\
\hline 1 & 13 \\
2 & 7 \\
3 & 8 \\
4 & 4 \\
5 & 2 \\
6 & 1 \\
\hline
\end{tabular}

Abbildung 8: Verlegungen der Niederschlagmeßstation „Sieber“ (DWD) im Harz mit einer Skizze des Dorfes und Höhenlinien; Position 1: von 7/46 bis 11/62, Position 2: 11/62 bis 11/63, Position 3: 11/63 bis 9/73, Position 4: seit $9 / 73$

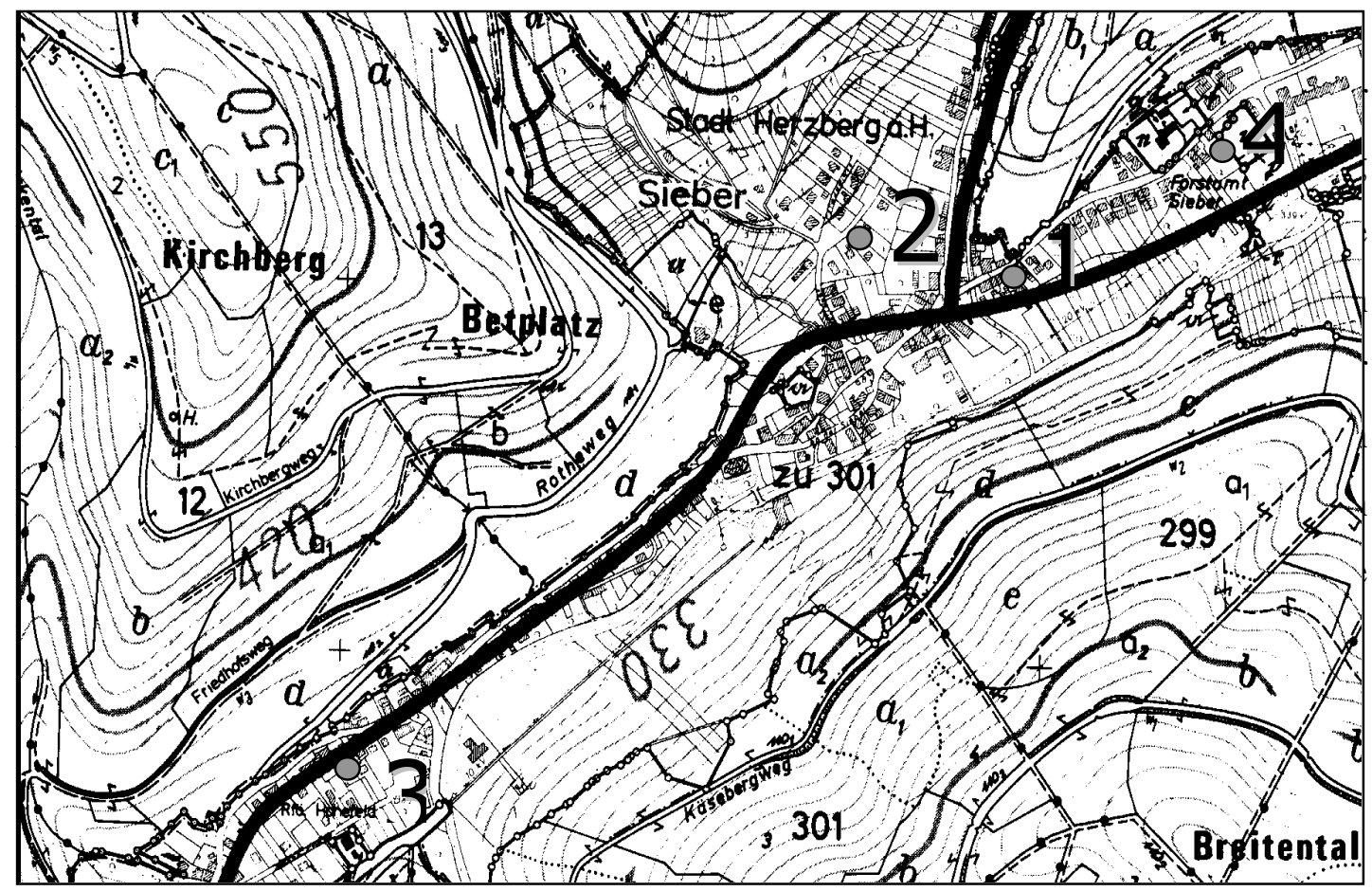


Tabelle 7: Meßorte der DWD-Niederschlagstation „Sieber“ im Beobachtungszeitraum 1961 bis 1990, beschrieben durch die für die Orte ermittelten Werte räumlicher Variablen

\begin{tabular}{|c|c|c|c|c|}
\hline & Pos. 1 & Pos. 2 & Pos. 3 & Pos. 4 \\
\hline Beobachtungsdauer & $\begin{array}{c}1,5 \text { Jahre } \\
7 / 46-11 / 62\end{array}$ & $\begin{array}{c}1 \mathrm{Jahr} \\
11 / 62-11 / 63\end{array}$ & $\begin{array}{c}9,5 \text { Jahre } \\
11 / 63-9 / 73\end{array}$ & $\begin{array}{l}17 \text { Jahre } \\
\text { seit } 9 / 73\end{array}$ \\
\hline $\begin{array}{l}\text { Geländehöhe } \\
\text { [m ü. NN] }\end{array}$ & 330,6 & 343,5 & 323,0 & 336,0 \\
\hline $\begin{array}{c}\text { Hangrichtung } \\
{\left[{ }^{\circ}\right]}\end{array}$ & 324,5 & 129,8 & 143,9 & 308,7 \\
\hline $\begin{array}{c}\text { Hangneigung } \\
{[\%]}\end{array}$ & 12,9 & 19,7 & 11,4 & 6,4 \\
\hline $\begin{array}{l}\text { Insolation Jan. } \\
\text { [ ] }\end{array}$ & 18,7 & 114,9 & 133,8 & 20,5 \\
\hline $\begin{array}{l}\text { Rel. Exponiertheit } \\
\quad(0 \text { bis } 36)\end{array}$ & 8,0 & 10,2 & 6,1 & 8,0 \\
\hline $\begin{array}{l}\text { Kaltluftabfluß } \\
\quad(0 \text { bis } 2000)\end{array}$ & 2000 & 829 & 2000 & 2000 \\
\hline $\begin{array}{l}\text { Leewirbel } \\
{[\mathrm{m}]}\end{array}$ & 597 & 676 & 682 & 542 \\
\hline $\begin{array}{l}\text { Lee-Effekt } 50 \\
{[\mathrm{~m}]}\end{array}$ & -16 & 0 & -95 & -9 \\
\hline $\begin{array}{l}\text { Abstand z- Meer } \\
{[\mathrm{m}]}\end{array}$ & 237241 & 237118 & 237510 & 237174 \\
\hline
\end{tabular}

Um zu überprüfen, ob die Berücksichtigung der Stationsverlegungen bei der Regionalisierung von Bedeutung ist, wurden alternativ zwei Regionalisierungsmodelle berechnet: Das erste berücksichtigte Werte der räumlichen Variablen, die für die aktuelle bzw. zuletzt bekannte Lage der Stationen ermittelt wurden. Es folgte eine schrittweise lineare Regression mit den in Kapitel 2.5 beschriebenen Bedingungen zur Einfachheit und Plausibilität. Der zweite Ansatz verfolgte das Ziel, Informationen über die Stationsverlegungen, bzw. die jeweiligen Positionen zu berücksichtigen. Dies geschah durch eine mit der Beobachtungsdauer gewichtete Mittelung der Variablenwerte einer jeden Position (z. B. Geländehöhe: 3 Positionen von 1961 bis 1990) für jede Station getrennt. Befand sich Station A fünf Jahre lang auf einer Geländehöhe von 210 m, danach 15 Jahre auf einer Geländehöhe von $220 \mathrm{~m}$ und die letzten zehn Jahre des Beobachtungszeitraums auf einer Höhe von $230 \mathrm{~m}$, so wurde $221,7 \mathrm{~m} \quad(=(5 * 210 \mathrm{~m}+15 * 220 \mathrm{~m}+10 * 230 \mathrm{~m}) / 30)$ in die stufenweise Regression als Wert der Variable Geländehöhe für Station A integriert. Die lineare Gewichtung führt somit zur Integration der Informationen über die Stationsverlegungen, ,ungewichtete Variablen" berücksichtigen diese nicht.

Bisherige Verlegungen der im Harz befindlichen Niederschlagmeßstationen wurden aus Skizzen und Lageplänen und aus Beschreibungen in den Stationsdokumenta- 
tionen des DWD rekonstruiert. Der Harz wurde in diesem Zusammenhang als Untersuchungsgebiet gewählt, weil er das Gebiet mit der größten orographischen Variabilität in ganz Niedersachsen ist. Wenn Verlegungen und ihre Erfassung von Bedeutung für die Regionalisierung sind, sollte dies hier zu zeigen sein. Zudem stand mit dem Datensatz der Harzwasserwerke (HWW) ein zweiter Datensatz für eine Validierung der DWD-Modelle zur Verfügung.

Bevor auf die Ergebnisse der Regressionen mit gewichteten und ungewichteten Variablen eingegangen wird, sollen die Unterschiede der beiden Variablenwerte anhand von zwei Tabellen dargestellt werden. In Tabelle 8 werden zunächst die Verteilungen von ungewichteten und gewichteten Variablenwerten der 35 Niederschlagmeßstationen im Harz dargestellt. Einige Variablen werden offensichtlich stärker durch die Gewichtung bzw. die Verlegungen beeinflußt als andere. So fallen Unterschiede zwischen den Verteilungen von gewichteten und ungewichteten Variablenwerten bei Leewirbel, Lee-Effekt 50 oder Kaltluftabflu $\beta$ deutlicher aus als z. B. bei Geländehöhe. Interessanter noch ist aber der direkte Vergleich für jede Station, die Differenz von gewichteten und ungewichteten Variablen. Deskriptive Statistiken zu diesen Differenzen sowie der einfache Korrelationskoeffizient nach Pearson zwischen gewichteten und ungewichteten Variablen sind in Tabelle 9 zusammengestellt.

Tabelle 8: Deskriptive Statistiken zu ungewichteten und gewichteten Variablenwerten von 35 DWD-Niederschlagmeßstationen im niedersächsischen Harz

\begin{tabular}{|c|c|c|c|c|}
\hline Variable & Mittelw. & Stdabw. & Min. & Max. \\
\hline \multirow{2}{*}{$\begin{array}{r}\text { Geländehöhe [m ü. NN] } \\
\text { gewichtet }\end{array}$} & 415,2 & 202,4 & 190,6 & 1132,0 \\
\hline & 415,4 & 204,4 & 192,1 & 1132,0 \\
\hline Hangneigung $\left[{ }^{\circ}\right]$ & 10,1 & 9,3 & 1,3 & 44,0 \\
\hline gewichtet & 11,3 & 9,3 & 1,3 & 42,6 \\
\hline Rel. Exponiertheit (0 bis 36$)$ & 27,8 & 11,0 & 0,6 & 36,0 \\
\hline gewichtet & 27,9 & 10,5 & 0,6 & 36,0 \\
\hline Exponiertheit z. (0 bis 36 ) & 11,9 & 9,7 & 0,4 & 33,4 \\
\hline gewichtet & 11,9 & 9,6 & 0,4 & 33,4 \\
\hline Leewirbel [m] & 213,3 & 160,2 & 4,4 & 573,5 \\
\hline gewichtet & 199,8 & 158,5 & 3,9 & 595,4 \\
\hline Lee-Effekt 50 [m] & $-8,7$ & 246,3 & $-607,6$ & 423,7 \\
\hline gewichtet & $-7,1$ & 249,6 & $-607,6$ & 438,5 \\
\hline Insolation Jan. [ ] & 107,8 & 43,9 & 0,0 & 254,9 \\
\hline gewichtet & 108,4 & 43,6 & 0,0 & 254,3 \\
\hline Kaltluftabfluß (0 bis 2000) & 215,4 & 499,3 & 0,4 & 2000,0 \\
\hline gewichtet & 272,8 & 520,5 & 0,8 & 2000,0 \\
\hline Abstand z. Küste [m] & 229465 & 11352 & 207387 & 250984 \\
\hline gewichtet & 229545 & 11430 & 207407 & 251148 \\
\hline Rechtswert [m] & 3598183 & 9904 & 3581500 & 3615343 \\
\hline \multirow{3}{*}{ Hochwert $[\mathrm{m}]$} & 3598241 & 9917 & 3581990 & 3615503 \\
\hline & 5738382 & 10727 & 5718768 & 5757240 \\
\hline & 5738325 & 10827 & 5718875 & 5757206 \\
\hline
\end{tabular}


Tabelle 9: Deskriptive Statistiken zu den Differenzen und Korrelationskoeffizienten $\left(r_{\mathrm{xy}}\right)$ zwischen ungewichteten und gewichteten Variablenwerten von 35 DWD-Niederschlagmeßstationen im niedersächsischen Harz

\begin{tabular}{lrrrrc}
\hline \multicolumn{1}{c}{ Variable } & Mittelw. & Stdabw. & Min. & Max. & $\mathbf{r}_{\mathbf{x y}}$ \\
\hline Geländehöhe [m ü. NN] & 0,12 & 11,74 & $-31,6$ & 30,2 & 0,998 \\
Hangrichtung [ ${ }^{\circ}$ ] & 30,36 & 45,88 & 0,0 & 172,4 & \\
Hangneigung [\%] & 1,24 & 4,24 & $-7,3$ & 15,5 & 0,897 \\
Rel. Exponiertheit (0 bis 36) & 0,10 & 2,29 & $-7,2$ & 6,2 & 0,979 \\
Exp. z. U.-min. (0 bis 36) & $-0,01$ & 2,70 & $-6,5$ & 8,5 & 0,961 \\
Leewirbel [m] & $-13,54$ & 57,82 & $-274,3$ & 83,8 & 0,934 \\
Lee-Effekt 50 [m] & 1,57 & 32,34 & $-62,9$ & 149,4 & 0,992 \\
Insolation Jan. [ ] & 0,55 & 21,70 & $-67,7$ & 49,7 & 0,877 \\
Kaltluftabfluß (0 bis 2000) & 58,86 & 213,18 & $-261,0$ & 803,1 & 0,914 \\
Abstand z. Küste [m] & 79,96 & 431,34 & $-939,5$ & 1862,3 & 0,999 \\
Rechtswert [m] & 58,29 & 389,25 & $-1163,6$ & 1538,4 & 0,999 \\
Hochwert [m] & $-57,32$ & 473,75 & $-2035,5$ & 990,9 & 0,999 \\
\hline
\end{tabular}

Der Mittelwert ist hier Ausdruck für den durchschnittlichen Unterschied zwischen gewichteten und ungewichteten Variablenwerten. Negative Werte wie im Fall der Variable Leewirbel zeigen dabei grundsätzlich höhere Werte bei den ungewichteten Variablen an, positive deuten auf durchschnittlich höhere gewichtete Werte hin. Aussagekräftiger für den Vergleich der Variablenwerte sind aber Standardabweichungen und Extremwerte. Diese zeigen, daß z. B. im Fall der Hangrichtung zum Teil erhebliche Abweichungen zwischen gewichteten und ungewichteten Variablenwerten bestehen. Auch sind die Differenzen für die Variable Leewirbel erheblich höher als die für Lee-Effekt 50, was in diesem Kapitel noch von Bedeutung sein wird.

Der Korrelationskoeffizient zwischen gewichteten und ungewichteten Variablenwerten kann als Index für die Ähnlichkeit von gewichteten und ungewichteten Variablenwerten und damit für die Stabilität der Variablen gegen Verlegungen verwendet werden. Er prüft dabei die Frage, ob höhere gewichtete Variablenwerte auch höhere ungewichtete Variablenwerte erwarten lassen. Für die Variable Hangrichtung macht die Berechnung dieser Größe keinen Sinn, da z. B. Wertepaare wie $355^{\circ}$ und $2^{\circ}$ trotz ähnlicher räumlicher Aussage zu einem geringen Korrelationskoeffizienten führen. Der Wert 0 bedeutet vollkommene Unabhängigkeit der Variablen, der Wert 1 eine absolute Korrelation. Derart stark korrelierte Variablen müssen das gleiche Erklärungspotential für eine dritte (abhängige) Variable besitzen. Die aufwendige Ermittlung gewichteter Variablenwerte kann entfallen. 
Der Unterschied zwischen Regressionsmodellen mit gewichteten und ungewichteten Variablenwerten bezüglich ihrer Anpassung an die Daten wird am besten deutlich durch eine Validierung, wofür ein zweiter unabhängiger Datensatz zur Verfügung stehen muß. Dieser muß auf vergleichbare Weise entstanden sein und denselben Zeitraum repräsentieren wie derjenige, der zur Parametrisierung des Modells dient. Zusätzlich zum Datenkollektiv der DWD-Niederschlagmeßstationen im Harz stand derjenige der Harzwasserwerke als Validierungsdatensatz zur Verfügung.

Der DWD unterhielt im Beobachtungszeitraum (1961 bis 1990) 35 Stationen mit einer Beobachtungsdauer von mindestens 25 Jahren im Harz, für die eventuelle Verlegungen nachvollzogen werden konnten. Weitere 67 Meßstationen der Harzwasserwerke konnten zur Validierung herangezogen werden. Schrittweise Regressionsanalyse der DWD-Daten führte für gewichtete und ungewichtete Variablen $\mathrm{zu}$ den in Tabelle 10 aufgeführten Modellen mit teilweise unterschiedlichen räumlichen Variablen. Unplausible Regressoren wurden eliminiert, wodurch für einige Monate mehrere Varianten entstanden, deren Qualität hier nicht diskutiert werden kann. Die letztlich nach der in Kap. 2.5 beschriebenen Methodik zu verwendenden Regionalisierungsmodelle wären die jeweils zuletzt aufgeführten, fett gedruckten Monatsmodelle. Interessant ist hier, wieviel Schritte bis zum Erreichen des finalen Modells jeweils nötig waren. 
Tabelle 10: Bestimmtheitsmaße $\left(\mathbf{R}^{2}\right)$ und Regressoren für Regressionsmodelle der langjährigen mittleren monatlichen und jährlichen Niederschlagssummen im Harz (35 DWD-Stationen) mit gewichteten und ungewichteten Variablenwerten

\begin{tabular}{l|cc|cc}
\hline $\begin{array}{c}\text { Monat/ } \\
\text { Jahr }\end{array}$ & \multicolumn{2}{|c|}{$\mathbf{R}^{2}[\%]$} & \multicolumn{2}{c}{ Regressoren } \\
gewichtet & ungewichtet & gewichtet & ungewichtet \\
\hline Jan & $\mathbf{8 2 , 1 3}$ & 81,14 & Geländehöhe, Leewirbel & Geländehöhe, LeeEffekt 50 \\
& & $\mathbf{7 5 , 1 1}$ & LeeEffekt 50 \\
Feb & 86,04 & 85,88 & Geländehöhe, expmin & Geländehöhe, expmin \\
& $\mathbf{8 5 , 6 5}$ & $\mathbf{7 9 , 4 1}$ & Geländehöhe, Leewirbel & Geländehöhe \\
Mär & $\mathbf{8 9 , 4 8}$ & 87,86 & Geländehöhe, Leewirbel & Geländehöhe, LeeEffekt 50 \\
& & $\mathbf{8 2 , 8 1}$ & Geländehöhe \\
Apr & $\mathbf{8 3 , 9 5}$ & $\mathbf{8 4 , 4 2}$ & LeeEffekt 50 & LeeEffekt 50 \\
Mai & 93,06 & 92,65 & LeeEffekt 50, Hochwert & LeeEffekt 50, Hochwert \\
& 91,97 & 91,88 & LeeEffekt 50, Abstand z. & LeeEffekt 50, Abstand z. \\
& & & Küste & Küste \\
& $\mathbf{8 5 , 3 3}$ & $\mathbf{8 3 , 6 2}$ & LeEffekt 50 & LeeEffekt 50 \\
Jun & $\mathbf{7 9 , 3 7}$ & $\mathbf{7 8 , 8 6}$ & LeeEffekt 50 & LeeEffekt 50 \\
Jul & $\mathbf{8 4 , 9 7}$ & $\mathbf{8 3 , 4 0}$ & LeeEffekt 50 & LeeEffekt 50 \\
Aug & $\mathbf{8 4 , 1 4}$ & $\mathbf{8 2 , 1 4}$ & LeeEffekt 50 & LeeEffekt 50 \\
Sep & $\mathbf{8 0 , 7 8}$ & $\mathbf{8 1 , 6 4}$ & LeeEffekt 50 & LeeEffekt 50 \\
Okt & $\mathbf{8 0 , 6 6}$ & $\mathbf{8 1 , 9 3}$ & LeeEffekt 50 & LeeEffekt 50 \\
Nov & $\mathbf{8 7 , 5 6}$ & 85,63 & Geländehöhe, Leewirbel & Geländehöhe, expmin \\
& & $\mathbf{7 9 , 4 7}$ & Geländehöhe \\
Dez & $\mathbf{8 1 , 1 7}$ & 80,31 & Geländehöhe, Leewirbel & Geländehöhe, LeeEffekt \\
& & $\mathbf{7 4 , 5 4}$ & & LeeEffekt 50 \\
\hline Jahr & $\mathbf{8 2 , 0 4}$ & $\mathbf{8 3 , 4 2}$ & LeeEffekt 50 & LeeEffekt 50 \\
\hline
\end{tabular}

Die gewichteten Variablenwerte können in den meisten Monaten einen größeren Teil der Varianz erklären als die ungewichteten Variablenwerte (s. Bestimmtheitsmaße in Tabelle 10). Lediglich für die Monate April, September, Oktober und November sowie für den Jahresniederschlag zeigten die Modelle der ungewichteten Variablen höhere Bestimmtheitsmaße.

Die mit gewichteten Variablen berechneten Regressionsmodelle zeigen einen saisonalen Effekt: Während in den Monaten von April bis Oktober der LeeEffekt die Variable mit dem größten Erklärungspotential ist, können im Winter Geländehöhe und Leewirbel den größten Teil der Streuung des Niederschlags in den DWD-Daten erklären. Dabei wurden lediglich für den Februar und den Mai nicht auf Anhieb die letztlich gültigen Modelle gefunden. Die ungewichteten Variablen führen im Sommer zu den gleichen ,optimalen" Regressoren, im Winterhalbjahr hat die Variable Leewirbel aber keine ebenso große Bedeutung wie im Falle der gewichteten Variablen. Leewirbel ist im Winter (November bis März) bei Verwendung der gewichteten Variablenwerte stets so bedeutsam für die Varianzaufklärung des Niederschlages, daß stets ein plausibles Regressionsmodell hoher Präzision unter Beteiligung dieser Variable gefunden wird. Die Tatsache, daß durch die Verwendung 
gewichteter Variablen plausiblere und konsistente Modelle auch mit empfindlicheren Variablen gefunden werden, ist ein Indiz für die Verbesserung des Erklärungspotentials durch die Integration von Informationen über Stationsverlegungen während der Beobachtungsperiode.

Um die Präzision der DWD-Modelle anhand einer Validierung vergleichen zu können, wurde die Differenz zwischen dem regionalisierten Schätzwert und dem Meßwert einer jeden HWW-Station berechnet. Deutlich geringere mittlere absolute Differenzen der Modelle mit gewichteten Variablen als diejenigen der Modelle mit ungewichteten Variablen könnten den Mehraufwand, der zu ihrer Ermittlung nötig ist, rechtfertigen. Die Werte der mittleren absoluten Differenzen sind in Tabelle 11 zusammen mit den Wurzeln der mittleren quadratischen Fehler (rooted mean squared error, RMSE) der Regressionen aufgeführt:

Tabelle 11: Rooted mean squared error (RMSE) der Regressionsmodelle als Maß der Präzision der DWD-Modelle; mittlere absolute Differenz zwischen Prognose und Meßwert der HWW-Stationen und Vergleich beider Validierungsergebnisse (Vorteil durch die Verwendung gewichteter Variablenwerte), in Klammern gesetzt für Modelle mit unterschiedlichen Regressoren

\begin{tabular}{|c|c|c|c|c|c|}
\hline \multirow[t]{2}{*}{$\begin{array}{c}\text { Monat/ } \\
\text { Jahr }\end{array}$} & \multirow{2}{*}{\multicolumn{2}{|c|}{$\begin{array}{l}\text { RMSE der Regressionen } \\
\qquad 1 / 10 \mathrm{~mm}] \\
\text { gewichtet ungewichtet }\end{array}$}} & \multirow{2}{*}{\multicolumn{2}{|c|}{\begin{tabular}{c} 
Mittel des Absoluts der \\
Differenz Prognose - Meßwert \\
\multicolumn{2}{c}{$[1 / 10 \mathrm{~mm}]$} \\
gewichtet $\quad$ ungewichtet
\end{tabular}}} & \multirow[t]{2}{*}{$\begin{array}{c}\text { Vorteil gewichtet } \\
{\left[\begin{array}{c}{[1 / 10 \mathrm{~mm}]} \\
\text { Spalte (5) - Spalte(4) }\end{array}\right.}\end{array}$} \\
\hline & & & & & \\
\hline Jan & 139,2 & $\begin{array}{l}143,0 \\
161,8\end{array}$ & 139,64 & $\begin{array}{l}166,06 \\
166,13\end{array}$ & $(26,49)$ \\
\hline $\mathrm{Feb}$ & $\begin{array}{l}86,0 \\
872\end{array}$ & $\begin{array}{r}86,5 \\
102,8\end{array}$ & $\begin{array}{l}90,26 \\
85,71\end{array}$ & $\begin{array}{r}91,21 \\
100,53\end{array}$ & $(14,82)$ \\
\hline Mär & 86,8 & $\begin{array}{r}93,2 \\
109,2\end{array}$ & 88,84 & $\begin{array}{l}116,62 \\
113,24\end{array}$ & $(24,40)$ \\
\hline Apr & 68,2 & 67,2 & 79,82 & 80,80 & 0,98 \\
\hline Mai & $\begin{array}{l}33,5 \\
36,1\end{array}$ & $\begin{array}{l}34,5 \\
36,3\end{array}$ & $\begin{array}{l}61,98 \\
62,55\end{array}$ & $\begin{array}{l}62,46 \\
63,17\end{array}$ & \\
\hline & 48,0 & 50,8 & 64,24 & 64,15 & $-0,09$ \\
\hline Jun & 65,9 & 66,7 & 87,06 & 87,15 & 0,09 \\
\hline Jul & 69,1 & 72,7 & 84,56 & 84,53 & $-0,03$ \\
\hline Aug & 58,6 & 62,1 & 75,09 & 74,93 & $-0,16$ \\
\hline Sep & 75,2 & 73,5 & 76,08 & 76,23 & 0,15 \\
\hline Okt & 92,5 & 89,4 & 101,37 & 102,49 & 1,12 \\
\hline Nov & 107,4 & $\begin{array}{l}115,4 \\
135,8\end{array}$ & 118,90 & $\begin{array}{l}123,61 \\
140,66\end{array}$ & $(21,76)$ \\
\hline Dez & 168,0 & $\begin{array}{l}171,8 \\
192,4\end{array}$ & 155,79 & $\begin{array}{l}187,98 \\
189,67\end{array}$ & $(33,88)$ \\
\hline Jahr & 1086,9 & 1044,5 & 1199,09 & 1214,46 & 15,37 \\
\hline
\end{tabular}

Insgesamt sind die Fehler aller Modelle als gering zu bezeichnen. Nur die Modelle der Monate Mai, Juli und August zeigen leichte Vorteile der Verwendung von ungewichteten Variablen. Alle anderen Vergleiche von Modellen mit identischen 
Regressoren belegen eine höhere Genauigkeit durch die Integration der Verlegungsinformationen. Alle gefundenen Unterschiede sind jedoch eher gering im Verhältnis zu der Höhe der Meßwerte. Die eingeklammerten Unterschiede für die Monate, für welche keine Modelle mit identischen Regressoren gefunden wurden, zeigen mit bis zu knapp $3.4 \mathrm{~mm}$ im Dezember deutlichere Unterschiede.

Zusammenfassend ist festzustellen, daß die Fehler der Regressionsmodelle im Verhältnis zu den Niederschlägen im Untersuchungsgebiet (hier: Harz) sehr gering sind. Eine treffsichere Regionalisierung von Niederschlägen auch für andere Teile Niedersachsens unter Anwendung der in Kapitel 2.5 beschriebenen Methodik kann erwartet werden. Der rooted mean squared error der Regressionsmodelle (RMSE) wies nur geringe Differenzen zum mittleren absoluten Schätzfehler des Validierungsdatensatzes auf und kann als guter Schätzer für die Genauigkeit der Modelle angesehen werden. Die Berücksichtigung der Stationsverlegungen durch die Verwendung von mit der Beobachtungsdauer gewichteten Variablenwerten machte sich vor allem in der größeren Konsistenz der Modelle bemerkbar. Kleinräumig veränderliche Variablen werden durch Verlegungen eher in ihrem Erklärungspotential eingeschränkt als stabilere Variablen. Werden die durch Verlegungen bewirkten Veränderungen der Meßsituation nicht berücksichtigt, kann der Informationsgehalt der Daten nicht vollständig genutzt werden, es ist mit suboptimalen Regionalisierungsmodellen zu rechnen.

Im Rahmen der vorliegenden Arbeit wurden die gewichteten Variablenwerte verwendet, wo sie zur Verfügung standen. Dies war bei allen Wetter- und Klimahauptstationen sowie den Niederschlagmeßstationen des DWD im Harz der Fall. Für alle anderen Niederschlagmeßstationen des DWD wurden ungewichtete Variablenwerte verwendet, da der zur Berechnung notwendige Aufwand für die Recherche der Stationsverlegungen im Verhältnis zum Informationsgewinn in den weniger stark reliefierten Gebieten außerhalb des Harzes zu groß erschien. Für die Zukunft wird der vom DWD eingeschlagene Weg, für alle Stationen die Lage auch in Form von Koordinaten zu erfassen, begrüßt, was eine optimierte räumliche Analyse der gewonnen Daten ermöglichen wird. 


\subsubsection{Lufttemperatur}

In diesem Kapitel soll neben den Meßmethoden der Lufttemperatur und grundlegenden Dingen zur Datenhaltung auch die Möglichkeit etwas genauer dargestellt werden, mit dem GIS die Repräsentanz verwendeter Datenkollektive für das jeweilige Untersuchungsgebiet zu beurteilen. Dies wird zunächst detailliert für das gesamte Temperaturdatenkollektiv des DWD für den Raum Niedersachsens vorgestellt. Wichtige räumliche Eigenschaften der DWD-Messungen werden dadurch aufgedeckt.

Die durchschnittliche Lufttemperatur in $2 \mathrm{~m}$ Höhe über der Erdoberfläche ist für Berg- und Tiefland Niedersachsens getrennt regionalisiert worden. Daher erfolgt die Diskussion der Repräsentanz der verwendeten Stationen in den folgenden Unterkapiteln getrennt nach Berg- und Tiefland Von den 45 Temperaturmeßstationen in Niedersachsen liegen $16 \mathrm{im}$ Bereich des Berglandes, 29 im Bereich des Tieflandes. Die getrennte Behandlung von Berg- und Tiefland schien wegen der extrem unterschiedlichen orographischen Situation in den beiden Untergebieten angebracht (vgl. Abbildung 16, S. 70). Dieser Eindruck kann durch die Unterschiedlichkeit der Verteilungen der Rasterwerte räumlicher Variablen belegt werden (s. Tabelle 41, S. 106). Auch die Ergebnisse der getrennten Regionalisierung (vgl. Kap. 5.1.1.1 und Kap. 5.1.1.2 Tiefland) sind derart verschieden, daß eine separate Analyse der Untergebiete plausibel und notwendig scheint. Anhand der Regionalisierungsergebnisse wird die Trennung in Berg- und Tiefland zu Beginn des Kapitels 5.1 ab S. 103 begründet und erläutert.

Für die Regionalisierung der Lufttemperatur im Bergland konnte neben den DWDDaten ein zusätzliches Datenkollektiv verwendet werden Es handelt sich dabei um Meßwerte, die an 13 Meßstationen durch das Institut für Bioklimatologie in den Jahren 1994 und 1995 ortsfern ermittelt wurden. Diese befinden sich an einem NordSüd-TRANSEKT durch den niedersächsischen Harz.

Temperaturwerte wurden vom DWD in Form von langjährigen Monats- und Jahresmittelwerten in $1 / 10{ }^{\circ} \mathrm{C}$ zur Verfügung gestellt. Beobachtungszeitraum waren die Jahre von 1961 bis 1990. Für die vorliegenden Untersuchungen wurden nur solche Stationen berücksichtigt, die mindestens für $25 \mathrm{Jahre}$ im Beobachtungszeitraum Werte geliefert hatten. 
Temperaturmessungen werden vom DWD standardmäßig an den Klimahauptstationen und an den hauptamtlichen Wetterstationen vorgenommen. Während die Klimastationen von ehrenamtlichen Mitarbeitern des DWD betreut werden, sind die Wetterstationen mit hauptamtlichen Mitarbeitern besetzt oder sie verfügen über eine automatisierte Meßvorrichtung. Sie führen Beobachtungen mit höherer zeitlicher Auflösung und/oder mit erweitertem Parametersatz durch (vgl. „http://www.dwd.de/research/klis/ger/3122.html\#Umfang der Daten“).

Die Lufttemperatur wird vom DWD in einer Thermometerhütte in $2 \mathrm{~m}$ Höhe über dem Erdboden gemessen (DWD, 1980). Hierzu stehen ein Stationsthermometer (Trockenes und Feuchtes Thermometer, die zusammen das Psychrometer bilden) sowie ein Maximumthermometer, ein Minimumthermometer und ein Aspirator, der zusammen mit dem Stationsthermometer bzw. Psychrometer zur Bestimmung der relativen Luftfeuchtigkeit verwendet wird. Die Messung der Temperatur erfolgt täglich an drei Terminen auf $1 / 10^{\circ} \mathrm{C}$ genau: $07 \mathrm{Uhr}$ MOZ (Morgentermin), $14 \mathrm{Uhr}$ MOZ (Mittagtermin) und 21 Uhr MOZ (Abendtermin). Aus den Messungen dieser drei Termine (T1 bis T3) wird vom DWD die Tagesdurchschnittstemperatur $\mathrm{T}_{\mathrm{m}}$ wie folgt berechnet:

$$
\mathrm{T}_{\mathrm{m}}=\frac{\mathrm{T}_{1}+\mathrm{T}_{2}+2 * \mathrm{~T}_{3}}{4}
$$

Der Fehler von Flüssigkeitsthermometern beträgt nach Häckel (1990) nicht mehr als $1 / 10{ }^{\circ} \mathrm{C}$, was das Ablesen der Temperatur mit eben dieser Genauigkeit rechtfertigt. Bei stündlichen Messungen, wie sie an synoptischen Stationen („Wetterstationen“) des DWD durchgeführt werden, kann die in ( 21 ) beschriebene Mittelung durch die Bildung des arithmetischen Mittels aller Tagesmeßwerte ersetzt werden. Die auf beide Arten ermittelten Werte können als kompatibel angenommen werden, wie auch Rechnungen mit vergleichbaren Datensätzen zeigten (SABOROWSKI, mdl. Mitteilung 1998).

Langjährige Mittelwerte der durchschnittlichen Lufttemperatur von insgesamt 45 Stationen konnten verwendet werden. Die Stationen sind gleichmäßig über ganz Niedersachsen verteilt (Abbildung 9). Einfache deskriptive Statistiken zu den Meßwerten sind in Tabelle 12 zusammengefaßt. Sie zeigen einen typischen Jahresgang der Temperatur und eine Standardabweichung von $1,1^{\circ} \mathrm{C}$ bis knapp über $1,3{ }^{\circ} \mathrm{C}$ über alle Monate hinweg. Die großen Differenzen zwischen Maxima und Minima der Werte, Bereiche von $5,9^{\circ} \mathrm{C}$ im Januar bis zu 7,4 ${ }^{\circ} \mathrm{C}$ im April, deuten auf 
stark unterschiedliche Meßsituationen hin. Unterschiede bezüglich der Kontinentalität und der Reliefierung an den Standorten (vgl. Abbildung 16, S. 70) erscheinen als wahrscheinliche Ursachen für diese großen Unterschiede.

Tabelle 12: Deskriptive Statistiken zu den durchschnittlichen Lufttemperaturen im Niedersachsendatenkollektiv des DWD

\begin{tabular}{lcrc|c|c}
\hline $\begin{array}{c}\text { Monat/ } \\
\text { Jahr }\end{array}$ & n obs & $\begin{array}{c}\text { Min. } \\
{\left[\mathbf{1 / 1 0}{ }^{\circ} \mathbf{C}\right]}\end{array}$ & $\begin{array}{c}\text { Max. } \\
{\left[\mathbf{1 / 1 0}^{\circ} \mathbf{C}\right]}\end{array}$ & $\begin{array}{c}\text { Mittelw. } \\
{\left[\mathbf{1 / 1 0}{ }^{\circ} \mathbf{C}\right]}\end{array}$ & $\begin{array}{c}\text { Stdabw. } \\
{\left[\mathbf{1 / 1 0}{ }^{\circ} \mathbf{C}\right]}\end{array}$ \\
\hline Jan & 45 & -42 & 17 & 3,1 & 12,40 \\
Feb & 45 & -42 & 22 & 8,0 & 12,60 \\
Mär & 45 & -24 & 48 & 34,9 & 13,13 \\
Apr & 45 & 9 & 83 & 71,1 & 13,01 \\
Mai & 45 & 57 & 129 & 119,0 & 12,63 \\
Jun & 45 & 88 & 160 & 150,1 & 12,61 \\
Jul & 45 & 103 & 174 & 164,0 & 12,21 \\
Aug & 45 & 105 & 171 & 162,2 & 11,59 \\
Sep & 45 & 77 & 146 & 132,5 & 11,96 \\
Okt & 45 & 47 & 109 & 93,6 & 11,12 \\
Nov & 45 & -5 & 63 & 46,7 & 13,02 \\
Dez & 45 & -30 & 32 & 16,1 & 12,95 \\
\hline Jahr & 45 & 29 & 94 & 83,5 & 11,89 \\
\hline
\end{tabular}

\section{Abbildung 9: Räumliche Verteilung der DWD-Temperaturstationen} (Wetterstationen und Klimahauptstationen) in Niedersachsen

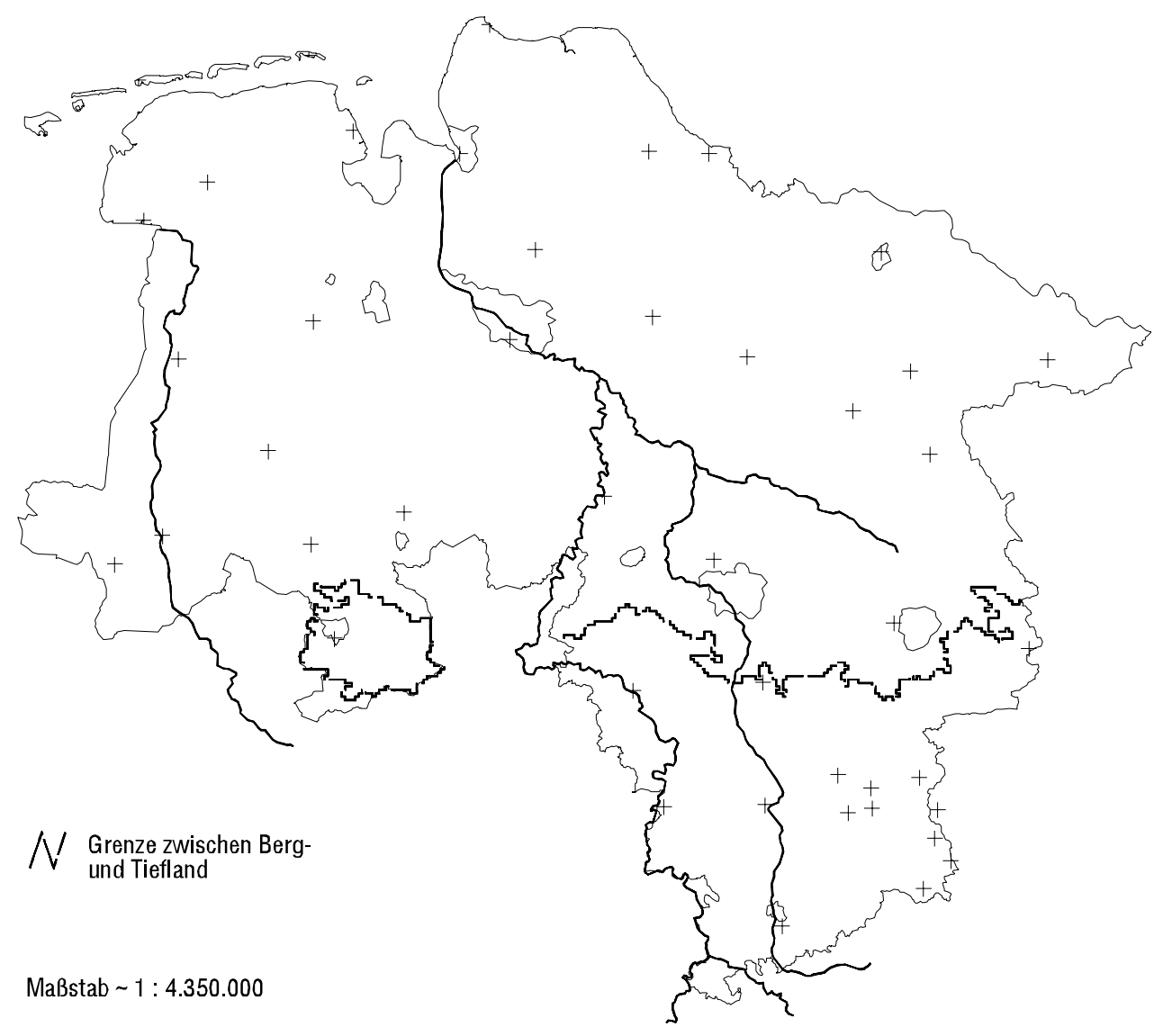


Bei der Auswahl seiner Stationen und der Plazierung der Meßvorrichtungen achtet der DWD i.a. darauf, daß die Meßergebnisse nicht von orographischen Besonderheiten, durch Vegetation oder Bebauung in der Stationsumgebung beeinflußt werden. Ziel ist es, Messungen durchzuführen, die untereinander gut vergleichbar sind, um so ein möglichst genaues Bild über die Verhältnisse in der Atmosphäre zu erhalten. Bezüglich der potentiellen Regressoren war folglich ein Mangel an Repräsentanz für diejenigen räumlichen Situationen zu erwarten, die vom Standard (mehr oder weniger ebene Lage ohne nennenswerte Horizontüberhöhungen) abweichen. Am deutlichsten wird dieser Umstand durch einen Vergleich der Stationsverteilung der Variable Hangneigung mit der Verteilung dieser Variable in ganz Niedersachsen, welche durch die Werte eines digitalen Höhenmodels (DHM) mit Rasterweite $50 \mathrm{~m}$ stichprobenartig beschrieben werden kann (s. Abbildung 10).

\section{Abbildung 10: Verteilung der Hangneigung der DWD-Temperaturstationen im Vergleich mit derjenigen für ganz Niedersachsen (Zellen eines digitalen Höhenmodells mit Rasterweite 50 m; DHM)}

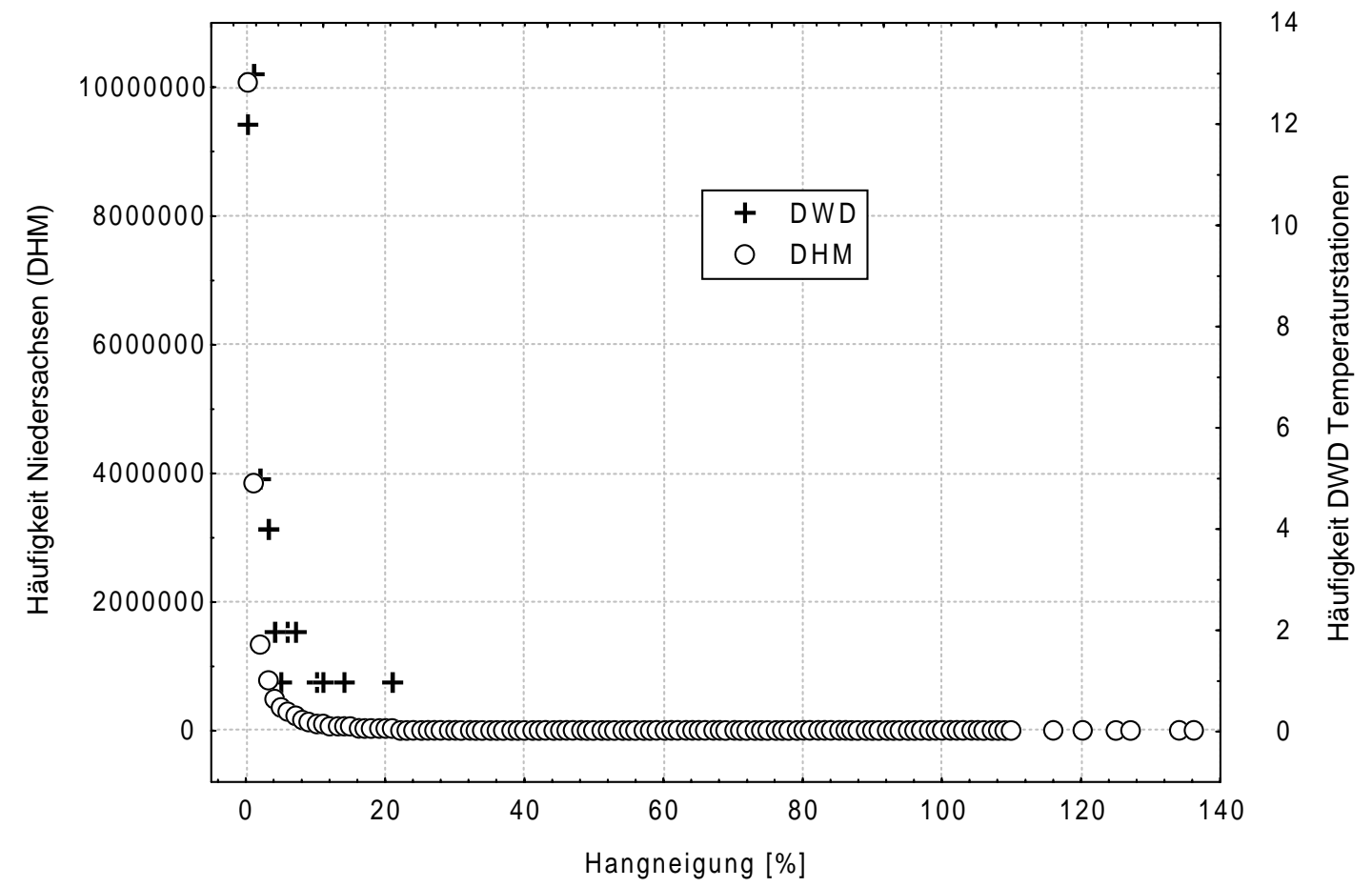

Der größte Teil der Stationen befindet sich in ebenem Gelände. Nur vier Stationen weisen eine Hangneigung von über $10 \%$ auf. Die steilste Hangneigung (Meßstation „,Bad Grund") ist mit 20,6 \% immer noch nicht als extrem zu bezeichnen. 
Den Beobachtungen bei der Hangneigung entsprechend weist das Temperaturdatenkollektiv des DWD auch keine Stationen im unteren Bereich des Wertespektrums der Variablen Relativen Exponiertheit auf (s. Abbildung 11). Den geringsten Wert für Relative Exponiertheit hat die Station in Bad Grund mit 15,1. Alle anderen Stationen liegen im oberen Bereich des Wertespektrums. Hier wird erneut Unterrepräsentanz der DWD-Stationen in Hang- und geschützten Tallagen dokumentiert.

Abbildung 11: Verteilung der Exponiertheit der DWD-Temperaturstationen mit derjenigen für ganz Niedersachsen (DHM mit Rasterweite $50 \mathrm{~m}$ ); Maximum von 36 aus Zwecken der Darstellung nicht berücksichtigt (DWD 38, DHM 17.907.922 Beobachtungen)

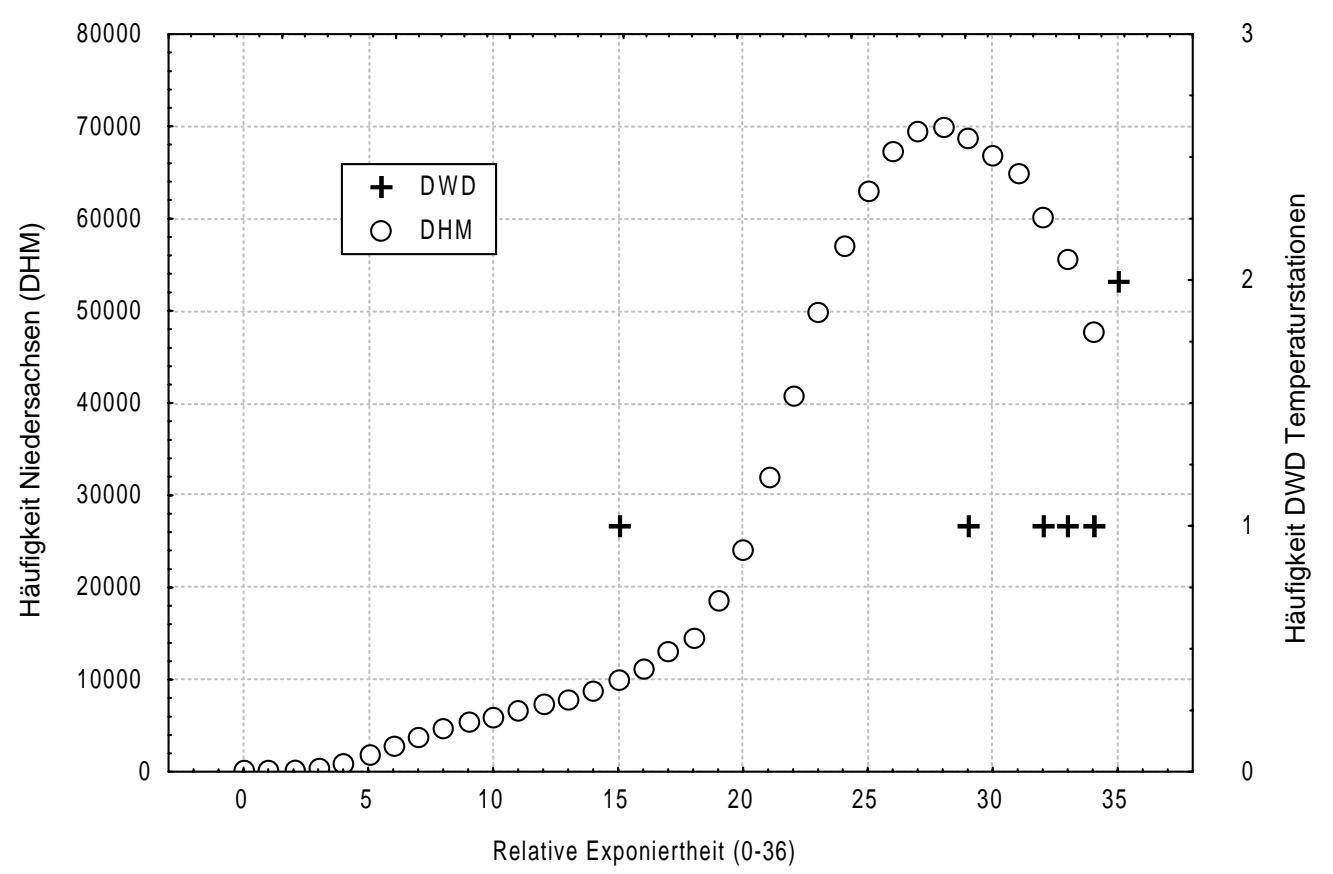

Für die Geländehöhe hingegen kann Repräsentanz des Datenkollektivs angenommen werden (s. Abbildung 12). Die Station „Brocken” (1142 m ü. NN) liegt über dem Maximum des Höhenmodells von Niedersachsen (953 m ü. NN). Dieser scheinbare Widerspruch läßt sich durch die Lage des Brockens auf dem Gebiet von SachsenAnhalt erklären. Wegen der Nähe zum Staatsgebiet Niedersachsens, der enormen Geländehöhe und der Beobachtung in Form einer Wetterstation erschien es dennoch sinnvoll und notwendig, die Ergebnisse dieser Station mit in das Datenkollektiv zu integrieren. 
Abbildung 12: Verteilung der Geländehöhe der DWD-Temperaturmeßstationen mit derjenigen für ganz Niedersachsen (DHM mit Rasterweite 50 m)

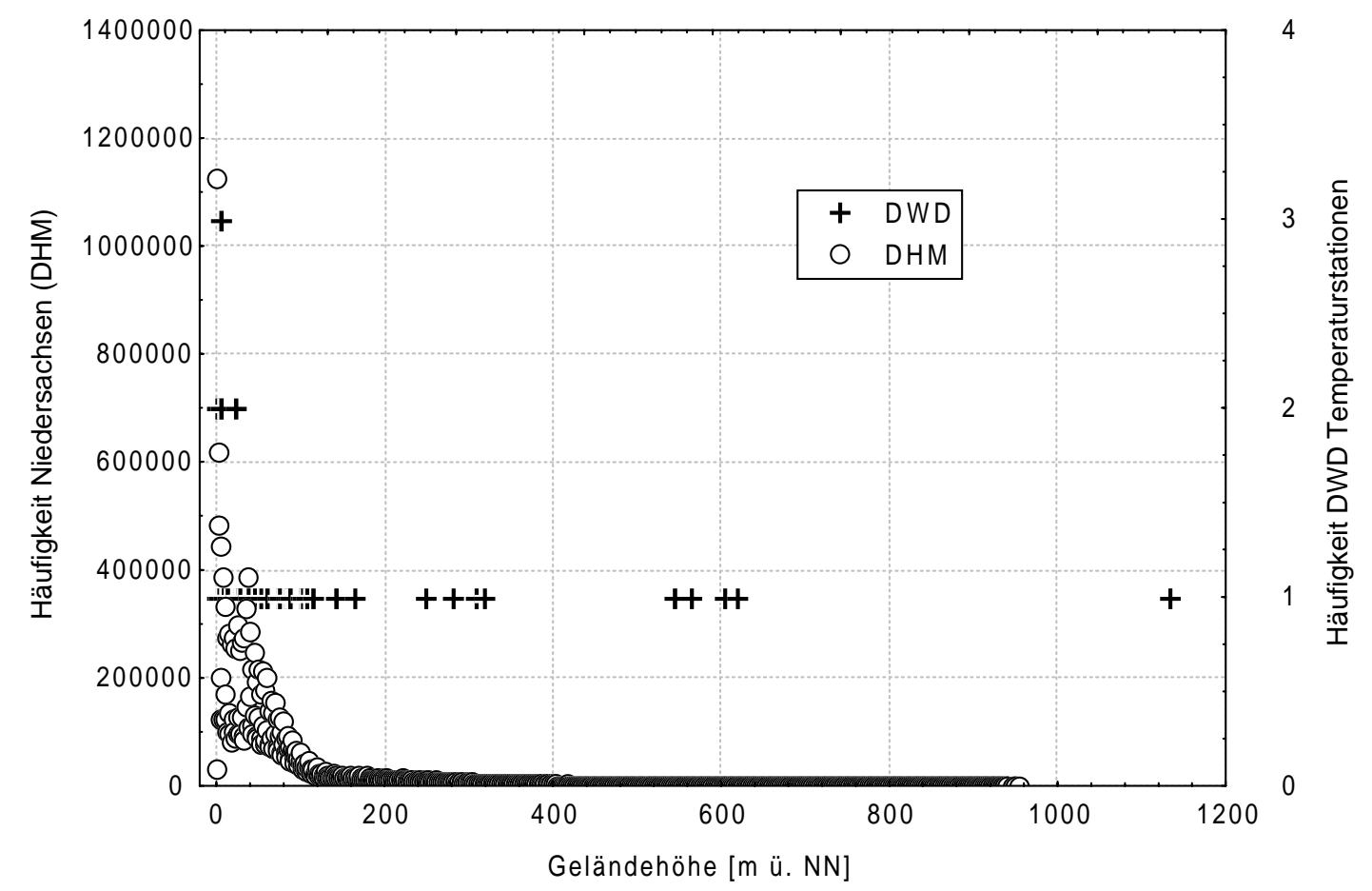

Eine weitere Variable, der Index für Kaltluftabfluß, wurde als zumindest teilweise repräsentativ eingestuft, da die Werte der Meßstationen einen großen Bereich der Werte des DHM abdecken konnten. Durch die geringe Belegung der einzelnen Klassen (vor allem der mit hohen Werten, vgl. Abbildung 13) und der Lücke von Werten zwischen 1000 und 2000 ist jedoch die Wahrscheinlichkeit relativ groß, daß der Einfluß einer solchen Variablen durch andere Einflüsse überdeckt oder falsch eingeschätzt werden kann. 
Abbildung 13: Verteilung des Indexes für Kaltluftabflu $\beta$ der Temperaturmeßstationen des DWD mit derjenigen für ganz Niedersachsen, vertreten durch die Zellen eines digitalen Höhenmodells (DHM) mit Rasterweite 50 m

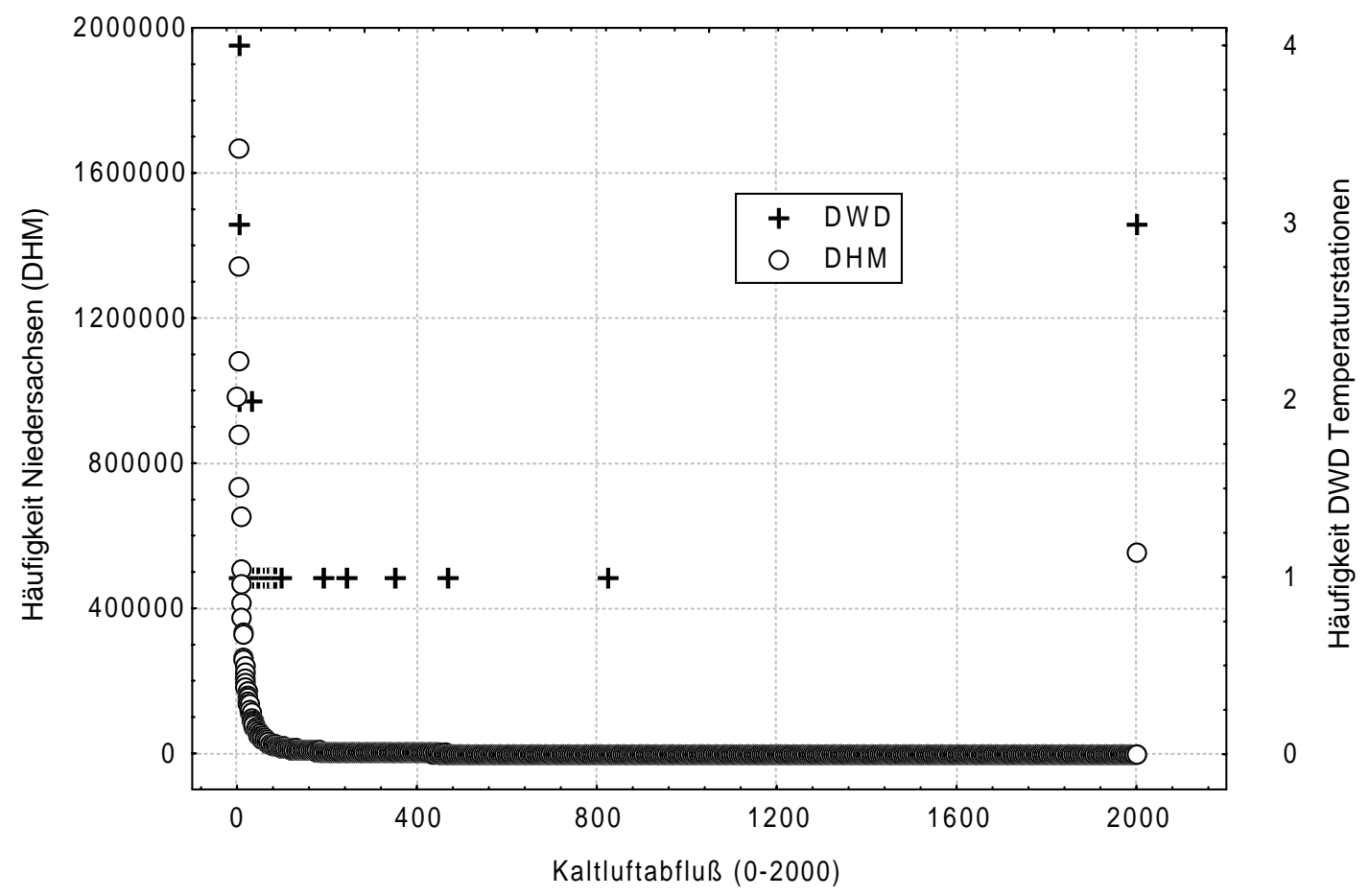

Aus dem bisher Beobachteten ist zu folgern, daß die DWD Temperaturstationen einen Mangel an Repräsentanz bezüglich der in Niedersachsen möglichen räumlichen Situationen zeigen. Um diesen Mangel zu quantifizieren wurde die Größe der Fläche, die nicht durch das jeweilige Datenkollektiv repräsentiert wird, mit Hilfe des GIS geschätzt (s. Tabelle 16 oder Tabelle 20).

\subsubsection{Lufttemperatur im Bergland}

Deskriptive Statistiken zu den hier verwendeten Datenkollektiven für monatliche und jährliche gemessene Durchschnittswerte der Lufttemperatur sollen einen Eindruck über die diesbezüglichen Verhältnisse im niedersächsischen Bergland geben. $\mathrm{Zu}$ beachten ist, daß die Stationen des TRANSEKT-Datenkollektivs allesamt zwischen $393 \mathrm{~m}$ ü. NN und $877 \mathrm{~m}$ ü. NN im Harz gelegen sind, während die 16 Stationen des DWD-Datenkollektivs über das gesamte niedersächsische Bergland verteilt sind. 
Tabelle 13: Deskriptive Statistiken zu den durchschnittlichen Lufttemperaturen im Berglanddatenkollektiv des DWD

\begin{tabular}{lcrrrrr}
\hline Monat/Jahr & n obs & $\begin{array}{c}\text { Min. } \\
\mathbf{1 / 1 0}{ }^{\circ} \mathbf{C}\end{array}$ & \multicolumn{1}{c}{$\begin{array}{c}\text { Max. } \\
\mathbf{1 / 1 0}{ }^{\circ} \mathbf{C}\end{array}$} & $\begin{array}{r}\text { Mittelw. } \\
\mathbf{1 / 1 0}^{\circ} \mathbf{C}\end{array}$ & $\begin{array}{c}\text { Stdabw. } \\
\mathbf{1 / 1 0}{ }^{\circ} \mathbf{C}\end{array}$ \\
\hline Jan & 16 & -42 & 11 & $-7,11$ & 14,74 \\
Feb & 16 & -42 & 17 & $-2,13$ & 15,89 \\
Mär & 16 & -24 & 45 & 26,14 & 18,71 \\
Apr & 16 & 9 & 83 & 64,74 & 20,01 \\
Mai & 16 & 57 & 129 & 112,98 & 19,12 \\
Jun & 16 & 88 & 160 & 143,96 & 19,10 \\
Jul & 16 & 103 & 174 & 158,13 & 18,89 \\
Aug & 16 & 105 & 171 & 155,71 & 17,42 \\
Sep & 16 & 77 & 139 & 124,88 & 16,94 \\
Okt & 16 & 47 & 100 & 85,60 & 14,35 \\
Nov & 16 & -5 & 54 & 36,37 & 16,39 \\
Dez & 16 & -30 & 24 & 5,25 & 15,41 \\
\hline Jahr & 16 & 29 & 92 & 75,46 & 17,05 \\
\hline
\end{tabular}

Beachtenswert erscheint neben dem klaren Jahresgang der Lufttemperatur auch die Tatsache, daß die Standardabweichung der Meßwerte im Frühling/Frühsommer ein Maximum erreicht, während im Winterhalbjahr eine deutlich geringere Varianz zu verzeichnen ist. Ähnliche Beobachtungen konnten auch für das TRANSEKTDatenkollektiv gemacht werden (Tabelle 14). Der Vergleich der Mittelwerte der beiden Kollektive fällt monatsweise unterschiedlich aus, was alleine schon durch den unterschiedlichen zeitlichen Bezug erklärt werden kann (DWD: Daten von 1961 bis 1990; TRANSEKT umfaßt Daten von 1994 und 1995). Daß die meisten Mittelwerte des DWD Berglandkollektivs über denen des TRANSEKT-Datenkollektivs liegen, ist nicht verwunderlich, da die TRANSEKT-Stationen allesamt im Harz auf über $390 \mathrm{~m}$ u. NN liegen (s.u.).

Tabelle 14: Deskriptive Statistiken zu den durchschnittlichen Lufttemperaturen im Berglanddatenkollektiv TRANSEKT

\begin{tabular}{lccccc}
\hline Monat/Jahr & n obs & $\begin{array}{c}\text { Min. } \\
{\left[\mathbf{1 / 1 0}{ }^{\circ} \mathbf{C}\right]}\end{array}$ & $\begin{array}{c}\text { Max } \\
{\left[\mathbf{1 / 1 0}{ }^{\circ} \mathbf{C}\right]}\end{array}$ & $\begin{array}{c}\text { Mittelw. } \\
{\left[\mathbf{1 / 1 0}{ }^{\circ} \mathbf{C}\right]}\end{array}$ & $\begin{array}{c}\text { Stdabw. } \\
{\left[\mathbf{1 / 1 0}{ }^{\circ} \mathbf{C}\right]}\end{array}$ \\
\hline Apr & 13 & 39 & 70 & 52,96 & 8,50 \\
Mai & 12 & 81 & 108 & 93,42 & 7,51 \\
Jun & 12 & 105 & 133 & 119,08 & 8,79 \\
Jul & 13 & 173 & 189 & 180,42 & 6,19 \\
Aug & 11 & 141 & 164 & 151,55 & 7,24 \\
Sep & 11 & 89 & 112 & 98,86 & 6,89 \\
Okt & 13 & 67 & 84 & 74,46 & 5,15 \\
Nov & 13 & 23 & 38 & 29,27 & 4,80 \\
Dez & 13 & -24 & -5 & $-15,65$ & 5,39 \\
\hline
\end{tabular}

Einen Überblick über den Vergleich zwischen den Verteilungen der DWD-Werte und der Rasterwerte verschiedener räumlicher Variablen gibt Tabelle 15 mit 
deskriptiven Statistiken. Vor allem der Vergleich der Extremwerte (Min. und Max.) kann einen Eindruck über Repräsentanzlücken des DWD- Meßnetzes geben. Mittelwerte und Standardabweichungen können in Verbindung mit den Extremwerten weiter Auskunft über Lage und Verteilung der Werte geben.

Tabelle 15: Gegenüberstellung von Variablenverteilungen (deskriptive Statistiken) für das Bergland (Rasterwerte) und die entsprechenden DWD Temperaturstationen

\begin{tabular}{|c|c|c|c|c|c|c|c|c|}
\hline \multicolumn{4}{|c|}{$\begin{array}{c}\text { Bergland } \\
\text { (Rasterwerte; Rasterweite: } \mathbf{5 0} \mathbf{~ m} \text { ) }\end{array}$} & \multirow[t]{2}{*}{ Variable } & \multicolumn{4}{|c|}{$\begin{array}{l}\text { DWD Temperaturstationen } \\
\text { im Bergland }(\mathrm{n}=16)\end{array}$} \\
\hline Min. & Max. & Mittelw. & Stdabw. & & Min. & Max. & Mittelw. & Stdabw. \\
\hline 35 & 953 & 218,23 & 130,75 & $\begin{array}{l}\text { Geländehöhe } \\
\text { [m ü. NN] }\end{array}$ & 72 & $1132^{2}$ & 337,35 & 288,80 \\
\hline 0 & 136 & 8,34 & 8,97 & $\begin{array}{c}\text { Hangneigung } \\
{[\%]}\end{array}$ & 1 & 21 & 5,90 & 5,34 \\
\hline 0 & 368 & 107,61 & 33,91 & $\begin{array}{c}\text { Insolation Januar } \\
{[\text { ] }}\end{array}$ & 63 & 162 & 111,19 & 22,02 \\
\hline 0 & 36 & 33,25 & 5,50 & $\begin{array}{l}\text { Rel. Exponiertheit } \\
\quad(0 \text { bis } 36)\end{array}$ & 15 & 36 & 33,61 & 5,35 \\
\hline 0 & 2000 & 92,25 & 311,76 & $\begin{array}{l}\text { Kaltluftabflu } \beta \\
(0 \text { bis } 2000)\end{array}$ & 1 & 2000 & 205,95 & 497,54 \\
\hline 0 & 1096 & 110,10 & 110,67 & $\begin{array}{c}\text { Leewirbel } \\
{[\mathrm{m}]}\end{array}$ & 0 & 262 & 94,33 & 87,50 \\
\hline
\end{tabular}

Nicht durch das DWD-Datenkollektiv erreichte Extremwerte des Rasters zeigen an, daß das Zielgebiet nicht vollständig vom Datenkollektiv repräsentiert werden kann. Der Anteil nicht repräsentierter räumlicher Situationen an der Gesamtfläche des Zielgebietes kann auch im Fall wertemäßig großer Repräsentanzlücken flächenmäßig durchaus vernachlässigbar gering sein. Einen groben Überblick über die Häufigkeiten entsprechender Werte außerhalb der Wertebereiche des Datenkollektivs können vegleichende Plots geben (vgl. Abbildung 10 oder Abbildung 13). Eine genaue Angabe der Häufigkeit von nicht vertretenen Rasterwerten läßt sich mit einem GIS ermitteln oder anhand der aus den Rastern gewonnenen Häufigkeitstabellen. Einen Überblick über das Ausmaß von solchen Repräsentanzmängeln der DWD- Temperaturstationen im Bergland gibt Tabelle 16.

2. Meßstation „Brocken“ befindet sich nicht in Niedersachsen aber in direkter Nachbarschaft 
Tabelle 16: Quantifizierung von Repräsentanzmängeln der DWD Temperaturstationen im niedersächsischen Bergland in Form der prozentualen Angabe von Rasterwerten, die nicht durch das Spektrum der Meßstationswerte abgedeckt werden

\begin{tabular}{cc}
\hline Variable & Repräsentanzmangel \\
\hline $\begin{array}{c}\text { Geländehöhe } \\
\text { [m ü. NN] }\end{array}$ & $2,4 \%$ unter Min. der Stationswerte \\
$\begin{array}{c}\text { Hangneigung } \\
\text { [\%] }\end{array}$ & $8,4 \%$ über Max. der Stationswerte \\
$\begin{array}{c}\text { Insolation Jan. } \\
\text { [ ] }\end{array}$ & $\begin{array}{c}13,2 \% \text { außerhalb des Bereichs der Stationswerte }(7,8 \% \text { kleiner, } 5,4 \% \\
\text { größer; Extremwerte Raster/Datenkollektiv unterscheiden sich stark) }\end{array}$ \\
$\begin{array}{c}\text { Rel. Exponiertheit } \\
\text { (0 bis 36) }\end{array}$ & $\begin{array}{c}1,6 \% \text { unter Min. der Stationswerte (Wert: } 0) \\
\text { Kaltluftabfluß } \\
(0 \text { bis } 2000) \\
\text { Leewirbel } \\
\text { [m] }\end{array}$ \\
\hline
\end{tabular}

Die größte Repräsentanzmangel ist demnach für die Variable Insolation im Januar festzustellen. Diese ist wahrscheinlich auf die gleichen Ursachen zurückzuführen wie die Einschränkung des Wertebereichs der Variable Hangneigung, nämlich die auf Vergleichbarkeit der Meßergebnisse ausgerichtete Auswahl der Meßstationen durch den DWD.

Ist ein Meßnetz bezüglich einer Variable nicht repräsentativ, so ist es möglich, daß existente Zusammenhänge zwischen dieser und einer oder mehreren Zielvariablen nicht durch die Analyse der Meßnetzdaten aufgedeckt werden können. Zu dieser Unsicherheit kommt das Problem, wie ein als signifikant erkannter (linearer) Zusammenhang zwischen einer Zielvariablen und einer nicht repräsentativ beobachteten räumlichen Variablen in der Regionalisierung zu verwenden ist. Es kann fallweise plausibel sein, auch für die nicht durch die Meßstationen vertretenen Werte den gleichen (linearen) Zusammenhang anzunehmen wie für das vorhandene Wertespektrum. Falls keine gegenteiligen Effekte - beispielsweise ein Sättigungseffekt - zu erwarten sind, kann es sinnvoll sein, einen gefundenen Zusammenhang auch über das von den Meßstationen abgedeckte Wertespektrum der räumlichen Variable hinaus zur Regionalisierung zu verwenden. Die Überprüfung des Wertebereichs der Regionalisierungsfunktion auf Plausibilität ist in diesem Fall unerläßlich.

Sollte ein anderer als der linear modellierte Zusammenhang für nicht repräsentierte Wertebereiche der räumlichen Variablen möglich oder gar wahrscheinlich sein, wird eine andere Vorgehensweise als die Extrapolation des Modells vorgeschlagen. Die 
Werte des Rasters, die über das durch die Stationen repräsentierte Spektrum hinausgehen (bzw. es unterschreiten), werden auf den maximalen (minimalen) Wert der Meßstationen gesetzt, die Variable wird gekappt. Gebiete, für die eine solche Kappung notwendig ist, können in Ergebnisplots beispielsweise durch Schraffur hervorgehoben werden. Dies ermöglicht dem fachlich versierten Nutzer die Möglichkeit bei relativ ungestörtem Gesamteindruck die möglichen Abweichungen in der Realität von der Modellierung und Darstellung zu bedenken und im Falle einer Verwendung bei weiterführenden Projekten wie z. B. einer automatisierten digitalen Standortbeschreibung zu berücksichtigen.

Aus dem Harz stand ein zweites Datenkollektiv zur Verfügung. Es war dies eine Meßreihe des Institutes für Bioklimatologie der Universität Göttingen, die an einem TRANSEKT durch den Harz von ca. 20 km Länge mit 15 Stationen über 2 Jahre (1994 und 1995) durchgeführt wurde. Stündlich wurden Werte an automatischen Meßstationen genommen, die jedoch durch die eingeschränkten Betreuungsmöglichkeiten im Winter (z.T. erhebliche Schneehöhen, Lage weitab von befahrbaren Straßen) nur für die Monate April bis Dezember zu monatlichen Mittelwerten integriert werden konnten. Die nördlichste Station 1 liegt nahe bei der Okertalsperre auf 443 m ü. NN. Das Transekt endet im Süden mit Station 15 auf 393 m ü. NN. Die Stationen 3 und 4 konnten leider keine vollständigen Meßreihen liefern, wodurch für die Regionalisierung nur 13 Stationen verwendet werden konnten. Die Lage der Stationen mit einem Eindruck vom Höhenprofil des beprobten Gebietes zeigt Abbildung 14. 
Abbildung 14: Lage der Stationen des TRANSEKT-Datenkollektivs an einem Nord-Süd-Transekt durch den Harz

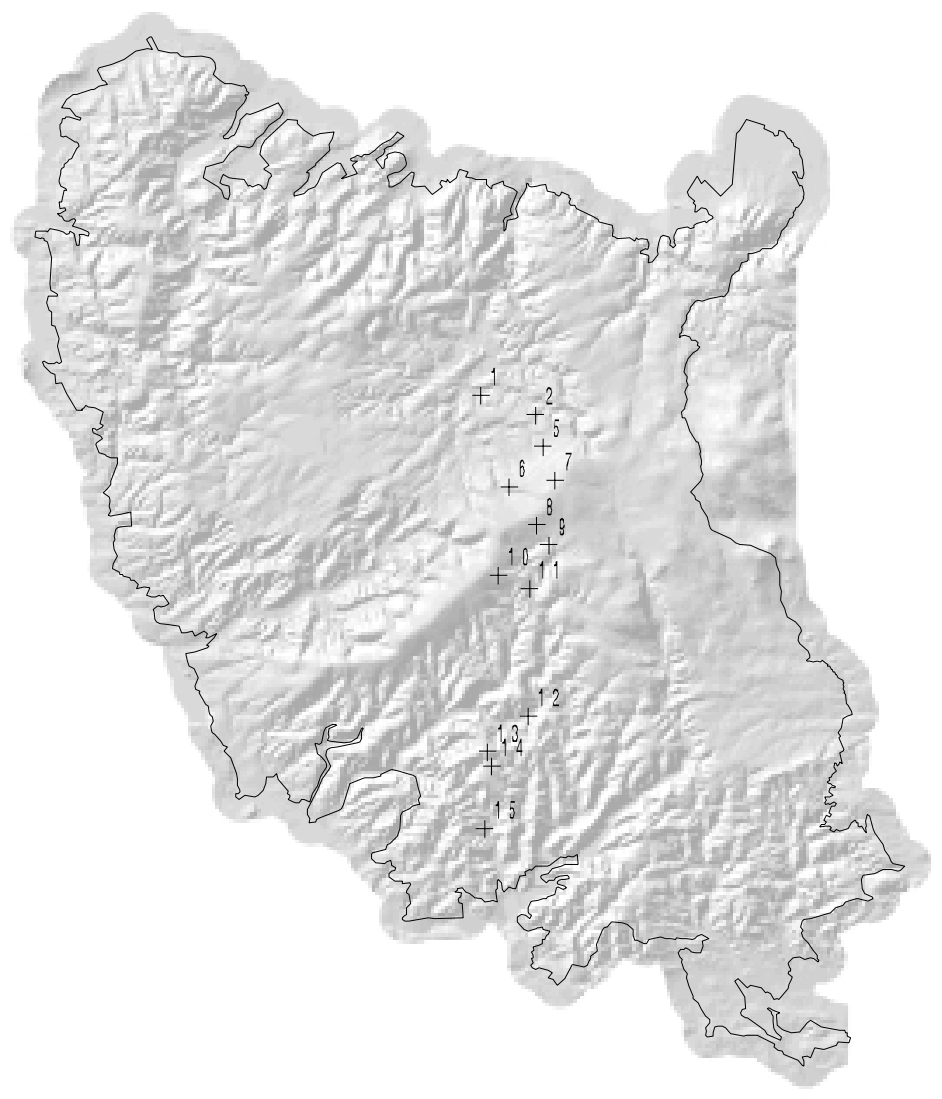

Für sich allein ist ein solches Datenkollektiv sicher nicht ausreichend für eine vollständige Regionalisierung der Lufttemperatur im Harz oder gar im gesamten niedersächsischen Bergland. Dies zeigt sich allein schon durch einen Vergleich der Verteilungen der Geländehöhe für das Datenkollektiv und den Harz (Abbildung 15). Die Werte von sechs räumlichen Variablen für alle 15 Stationen des TRANSEKTDatenkollektivs sind in Tabelle 17 zusammengefaßt. Eine genaue Betrachtung verdeutlicht schnell den Unterschied $\mathrm{zu}$ dem vorher besprochenen DWDDatenkollektiv. Während die Repräsentanz bezüglich der Geländehöhe schlechter ist als im Fall der DWD-Stationen, ist das Wertespektrum des TRANSEKTDatenkollektivs andere räumliche Variablen betreffend weiter gestreut als das des DWD-Datenkollektivs. 
Abbildung 15: Verteilung der Variable Geländehöhe der Temperaturmeßstationen des TRANSEKT-Datenkollektivs im Harz mit derjenigen für den gesamten niedersächsischen Harz, vertreten durch die Zellen eines digitalen Höhenmodells (DHM) mit Rasterweite $50 \mathrm{~m}$

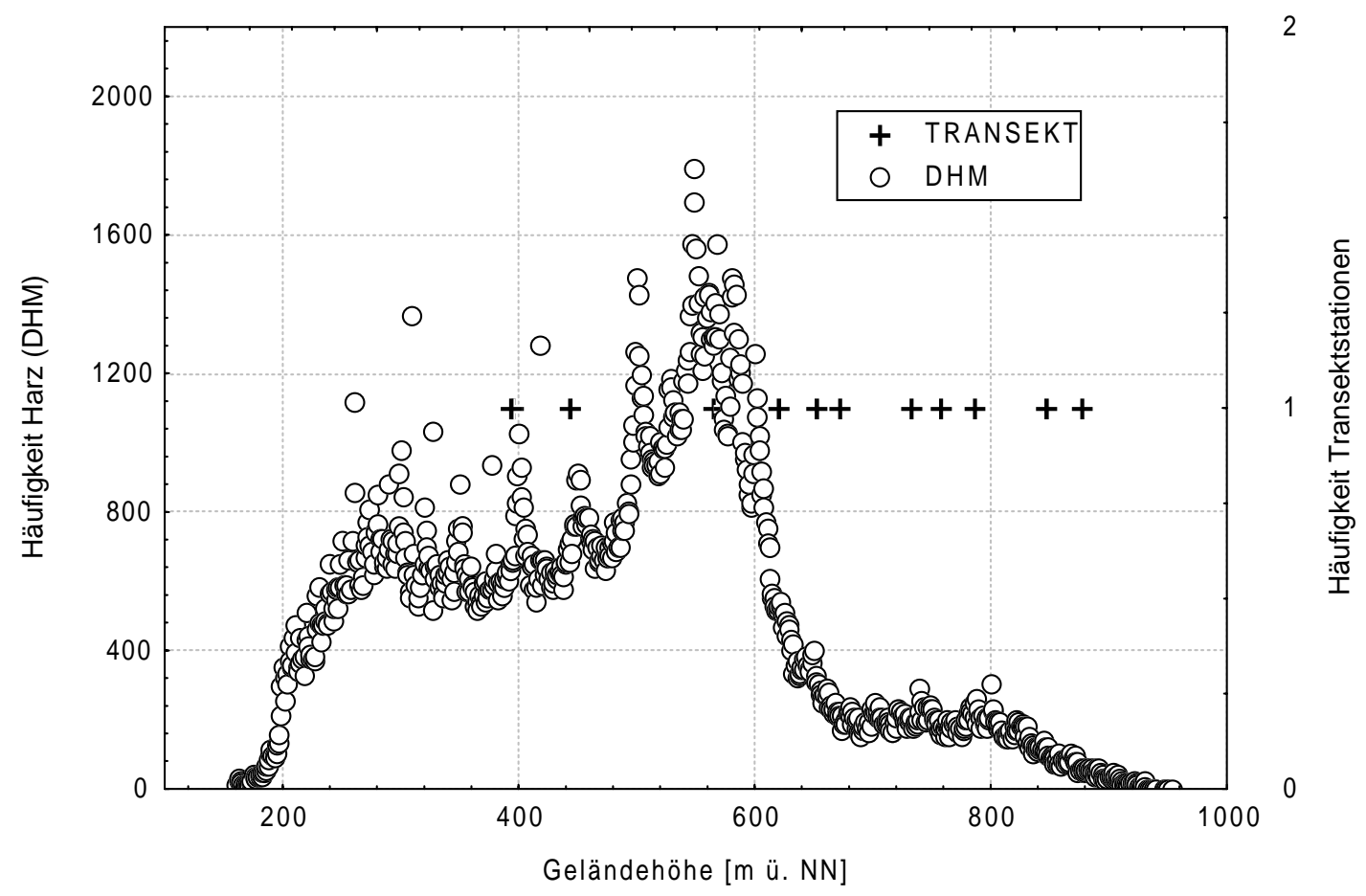

Tabelle 17: Werte von sechs beispielhaft ausgewählten räumlichen Variablen der 13 in der Untersuchung verwendeten Stationen des TRANSEKTDatenkollektivs im Harz

\begin{tabular}{rcccccr}
\hline Station & $\begin{array}{c}\text { Gelände- } \\
\text { höhe } \\
\text { [m ̈̈. NN] }\end{array}$ & $\begin{array}{c}\text { Insolation } \\
\text { Jan. } \\
{[\text { ] }}\end{array}$ & $\begin{array}{c}\text { Hang- } \\
\text { neigung } \\
\text { [\%] }\end{array}$ & $\begin{array}{c}\text { Relative } \\
\text { Exponiertheit } \\
\text { (0 bis 36) }\end{array}$ & $\begin{array}{c}\text { Kaltluft- } \\
\text { abfluß } \\
\text { (0 bis 2000) }\end{array}$ & $\begin{array}{c}\text { Leewirbel } \\
\text { [m] }]\end{array}$ \\
\hline 1 & 443 & 35 & 20 & 18 & 455 & 205 \\
2 & 565 & 170 & 29 & 29 & 7 & 114 \\
5 & 651 & 80 & 12 & 29 & 16 & 0 \\
6 & 733 & 98 & 5 & 36 & 1 & 6 \\
7 & 877 & 51 & 18 & 25 & 5 & 0 \\
8 & 846 & 173 & 17 & 29 & 3 & 141 \\
9 & 757 & 77 & 16 & 36 & 1 & 125 \\
10 & 785 & 114 & 10 & 36 & 1 & 215 \\
11 & 574 & 143 & 16 & 7 & 548 & 597 \\
12 & 395 & 65 & 1 & 8 & 2000 & 525 \\
13 & 671 & 26 & 22 & 24 & 2 & 46 \\
14 & 620 & 110 & 6 & 36 & 1 & 124 \\
15 & 393 & 217 & 37 & 9 & 13 & 685 \\
\hline
\end{tabular}

Für die Variable Insolation im Januar wurden so beispielsweise Werte deutlich unter dem Minimum des DWD-Datenkollektivs von 63 ermittelt (vgl. Tabelle 15, S. 64). Die Werte für Kaltluftabfluß (Stationen 1, 11 und 12) sowie die der Variable Leewirbel (Station 15!) zeigen eine große Streuung. Besonders deutlich wird der 
Unterschied zum DWD-Datenkollektiv jedoch mit den Variablen Hangneigung und Relative Exponiertheit. Hier zeigen die hohen Werte für Hangneigung und die geringen Werte für die Relative Exponiertheit, daß bei der Auswahl der Meßstandorte hier besonders auf die Erfassung von räumlichen Unterschieden Wert gelegt wurde. Ein Umstand, welcher der Regionalisierung unter Zuhilfenahme von räumlichen Variablen entgegenkommt. Trotz des unterschiedlichen zeitlichen Rahmens der beiden Datenkollektive wurde daher eine integrierte Analyse auf dem in Kap. 5.1.1.1 auf Seite 108f erläuterten Weg durchgeführt.

\subsubsection{Lufttemperatur im Tiefland}

Auch für das niedersächsische Tiefland sollen deskriptive Statistiken zu dem hier verwendeten Datenkollektiv für monatlich und jährlich gemessene Durchschnittswerte der Lufttemperatur einen Eindruck über die Verhältnisse im Zielgebiet geben:

Tabelle 18: Deskriptive Statistiken zu den durchschnittlichen Lufttemperaturen im Tieflanddatenkollektiv des DWD

\begin{tabular}{lcrrrc}
\hline Monat/Jahr & $\mathbf{n}$ obs & $\begin{array}{c}\text { Min. } \\
{\left[\mathbf{1 / 1 0}^{\circ} \mathbf{C}\right]}\end{array}$ & $\begin{array}{c}\text { Max. } \\
{\left[\mathbf{1 / 1 0}{ }^{\circ} \mathbf{C}\right]}\end{array}$ & $\begin{array}{c}\text { Mittelw. } \\
{\left[\mathbf{1 / 1 0}{ }^{\circ} \mathbf{C}\right]}\end{array}$ & $\begin{array}{c}\text { Stdabw. } \\
{\left[\mathbf{1 / 1 0}{ }^{\circ} \mathbf{C}\right]}\end{array}$ \\
\hline Jan & 29 & -4 & 17 & 8,69 & 5,78 \\
Feb & 29 & 3 & 22 & 13,52 & 4,89 \\
Mär & 29 & 32 & 48 & 39,79 & 3,85 \\
Apr & 29 & 68 & 82 & 74,62 & 3,94 \\
Mai & 29 & 112 & 129 & 122,24 & 4,84 \\
Jun & 29 & 144 & 159 & 153,41 & 4,66 \\
Jul & 29 & 160 & 172 & 167,17 & 3,59 \\
Aug & 29 & 159 & 170 & 165,83 & 3,26 \\
Sep & 29 & 129 & 146 & 136,66 & 4,45 \\
Okt & 29 & 89 & 109 & 98,03 & 5,19 \\
Nov & 29 & 42 & 63 & 52,38 & 5,29 \\
Dez & 29 & 10 & 32 & 22,10 & 5,63 \\
\hline Jahr & 29 & 81 & 94 & 87,93 & 3,06 \\
\hline
\end{tabular}

Deutlich ist der Jahresgang der Temperaturen sowohl bei den Extremwerten als auch beim Mittelwert zu erkennen. Die Standardabweichung der gemessenen Temperaturen zeigt eine geringere Varianz der Werte im Sommer als in den Wintermonaten. Im Vergleich zu den Berglandstationen (s. Tabelle 13) sind für das Tiefland deutlich höhere Temperaturen gemessen worden, die Werte der Standardabweichung sind bei den Berglandstationen deutlich höher. 
Eine ausführliche verbale Beschreibung der Verhältnisse im niedersächsischen Tiefland kann beispielsweise der vom ARBEITSKREIS STANDORTKARTIERUNG (1985) herausgegebenen Beschreibung der Wuchsgebiete und -bezirke für das Gebiet der damaligen Bundesrepublik Deutschland entnommen werden. Einen Eindruck der Reliefierung des Testgebietes gibt Abbildung 16. Zur Lage der Stationen sei erneut auf Abbildung 9, Seite 58 verwiesen.

\section{Abbildung 16: Reliefierung Niedersachsens mit der aus räumlichen Informationslagen im GIS abgeleiteten Grenze zwischen Berg- und Tiefland}

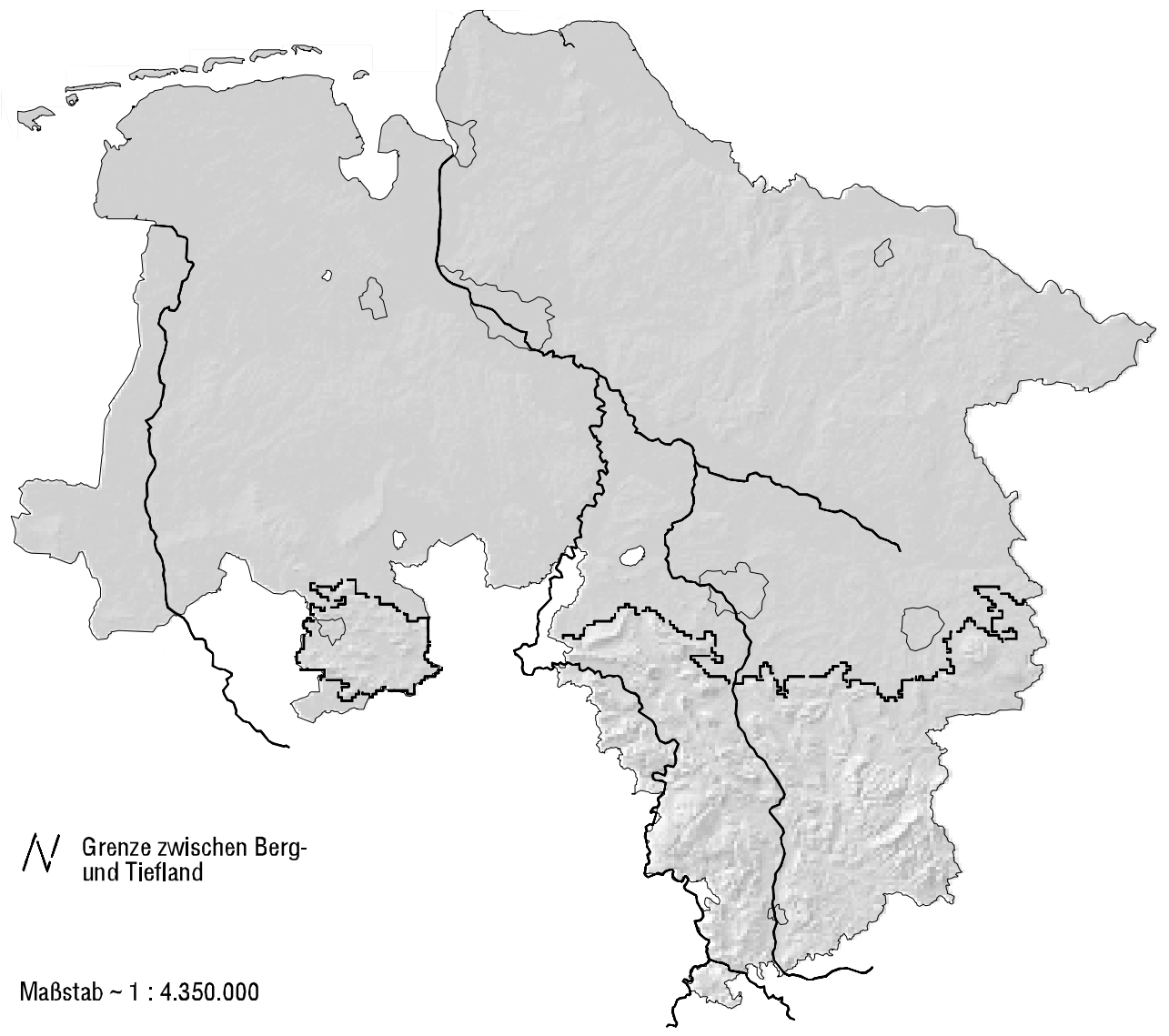

Die Extrema von Rastern und Werten des Datenkollektivs fast aller in Tabelle 19 dargestellten räumlichen Variablen weisen auf erhebliche Repräsentanzlücken des DWD-Temperaturdatenkollektivs im niedersächsischen Tiefland hin. Insgesamt zeigen die Werte in Tabelle 20 zwar einen kleineren Anteil nicht repräsentierter Flächen an als im Fall der Berglandstationen, aber die Probleme, die für den Fall erheblicher Differenzen zwischen den Maxima der Verteilungen von Rastern und Stationswerten in Kap. 4.1.2.1 für das Bergland diskutiert wurden, bestehen auch für 
die Tieflandstationen. Dies ist beispielsweise für die Variablen Leewirbel und Insolation im Januar aus Tabelle 19 zu ersehen. Eine erschreckend niedrige Varianz zeigt die Variable Relative Exponiertheit im Tiefland.

Tabelle 19: Gegenüberstellung von Variablenverteilungen (deskriptive Statistiken) für Tiefland und entsprechende DWD Temperaturstationen

\begin{tabular}{|c|c|c|c|c|c|c|c|c|}
\hline \multicolumn{4}{|c|}{$\begin{array}{c}\text { Tiefland } \\
\text { (Rasterwerte; Rasterweite: } \mathbf{5 0} \mathbf{~ m} \text { ) }\end{array}$} & \multirow[t]{2}{*}{ Variable } & \multicolumn{4}{|c|}{$\begin{array}{l}\text { DWD Temperaturstationen } \\
\text { im Tiefland }(n=29)\end{array}$} \\
\hline Min. & Max. & Mittelw. & Stdabw. & & Min. & Max. & Mittelw. & Stdabw. \\
\hline-11 & 172 & 35,53 & 28,35 & $\begin{array}{l}\text { Geländehöhe } \\
\text { [m ü. NN] }\end{array}$ & 0 & 101 & 26,15 & 29,69 \\
\hline 0 & 63 & 0,68 & 1,34 & $\begin{array}{c}\text { Hangneigung } \\
{[\%]}\end{array}$ & 0 & 10 & 1,22 & 1,95 \\
\hline 0 & 306 & 108,95 & 5,06 & $\begin{array}{c}\text { Insolation Januar } \\
{[]}\end{array}$ & 70 & 117 & 108,41 & 8,18 \\
\hline 0 & 36 & 35,99 & 0,28 & $\begin{array}{l}\text { Relative Exponiertheit } \\
\qquad(0 \text { bis } 36)\end{array}$ & 35 & 36 & 35,97 & 0,14 \\
\hline 0 & $\begin{array}{r}200 \\
0\end{array}$ & 138,01 & 398,09 & $\begin{array}{l}\text { Kaltluftabfluß } \\
(0 \text { bis } 2000)\end{array}$ & 1 & 2000 & 198,80 & 521,91 \\
\hline 0 & 406 & 12,04 & 18,74 & $\begin{array}{l}\text { Leewirbel } \\
{[\mathrm{m}]}\end{array}$ & 0 & 76 & 13,11 & 17,07 \\
\hline
\end{tabular}

Tabelle 20: Quantifizierung der Repräsentanzmängel der DWD Temperaturstationen im Tiefland in Form der prozentualen Angabe von Rasterwerten, die nicht durch das Spektrum der Meßstationswerte abgedeckt werden

\begin{tabular}{|c|c|}
\hline Variable & Repräsentanzmangel \\
\hline $\begin{array}{l}\text { Geländehöhe } \\
\text { [m ü. NN] }\end{array}$ & $1,8 \%$ über max. der Stationswerte \\
\hline $\begin{array}{c}\text { Hangneigung } \\
{[\%]}\end{array}$ & $\begin{array}{c}0,2 \% \text { über max. der Stationswerte }(10 \%) \text {; max. der } \\
\text { Rasterwerte: } 63 \%\end{array}$ \\
\hline $\begin{array}{l}\text { Insolation Jan. } \\
\text { [ ] }\end{array}$ & $\begin{array}{r}\text { 3,9\% außerhalb des Bereichs der Stationswerte, } \\
\text { Extremwerte unterscheiden sich stark }\end{array}$ \\
\hline $\begin{array}{l}\text { Rel. Exponiertheit } \\
\text { (0 bis } 36)\end{array}$ & $0,14 \%$ außerhalb des Bereichs der Stationswerte \\
\hline $\begin{array}{l}\text { Kaltluftabfluß } \\
(0 \text { bis } 2000)\end{array}$ & $\begin{array}{l}4,4 \% \text { unter min. der Stationswerte (Anteil von } \\
\text { Wert 0) }\end{array}$ \\
\hline $\begin{array}{l}\text { Leewirbel } \\
{[\mathrm{m}]}\end{array}$ & $\begin{array}{l}2,0 \% \text { über max. der Stationswerte }(76 \mathrm{~m}) \text {; max. der } \\
\text { Rasterwerte: } 406 \mathrm{~m}\end{array}$ \\
\hline
\end{tabular}

Die Varianz der Geländehöhe ist im Tiefland viel geringer als im Bergland, aber auch für andere räumliche Variablen, zumal die höhenabhängigen, wurden sehr kleine Standardabweichungen berechnet. Durch die räumliche Verteilung der Meßstationen (s. Abbildung 9, S. 58) ist zumindest eine große Varianz der lageabhängigen Variablen garantiert. Insgesamt lassen die zur Verfügung stehenden räumlichen Variablen und die geringen flächenrelativen Repräsentanzmängel (s. Tabelle 20) erwarten, daß auf dem in Kap. 2.5 beschriebenen Weg 
Regionalisierungsmodelle gefunden werden können, die für den größten Teil des Untersuchungsgebietes niedersächsisches Tiefland plausibel und gültig sind.

\subsubsection{Niederschlagssumme}

Die Datenbasis zur Regionalisierung der monatlichen und jährlichen Niederschlagssummen wurde aus zwei Meßnetzen zusammengestellt, dem des Deutschen Wetterdienstes (DWD) und dem der Harzwasserwerke (HWW). Der DWD mißt den täglichen Niederschlag sowohl an Wetter- und Klimahauptstationen (vgl. Kap. 4.1.2) als auch an ehrenamtlich betreuten Niederschlagmeßstationen. Die Meßstationen der HWW werden zum Monitoring und zur Vorhersage des Wasserstandes an 6 Staudämmen zur Trinkwassergewinnung betrieben. Standardmeßgerät ist bei beiden Meßnetzen der Hellmann-Regenmesser. Die Meßmethodik der DWD- und der HWW-Stationen ist absolut vergleichbar und drückt sich auch durch die Aufnahme einiger HWW-Stationen in das Meßnetz des DWD aus. Diese Stationen werden in der vorliegenden Untersuchung als DWDStationen verwendet. Bei einer ausreichend langen Beobachtungsperiode von mindestens 25 Jahren im Untersuchungszeitraum 1961 bis 1990 wurden die restlichen Stationen als HWW-Stationen geführt.

Durch die erhebliche Verdichtung des DWD-Niederschlagmeßnetzes gegenüber dem Temperaturmeßnetz durch von Laien betreute Stationen (Niederschlagmeßstationen) ist die geographische Verteilung aller hier zur Verfügung stehenden Stationen über ganz Niedersachsen als repräsentativ anzusehen. Einen Überblick gibt Abbildung 17. 
Regionalisierungsmodelle gefunden werden können, die für den größten Teil des Untersuchungsgebietes niedersächsisches Tiefland plausibel und gültig sind.

\subsubsection{Niederschlagssumme}

Die Datenbasis zur Regionalisierung der monatlichen und jährlichen Niederschlagssummen wurde aus zwei Meßnetzen zusammengestellt, dem des Deutschen Wetterdienstes (DWD) und dem der Harzwasserwerke (HWW). Der DWD mißt den täglichen Niederschlag sowohl an Wetter- und Klimahauptstationen (vgl. Kap. 4.1.2) als auch an ehrenamtlich betreuten Niederschlagmeßstationen. Die Meßstationen der HWW werden zum Monitoring und zur Vorhersage des Wasserstandes an 6 Staudämmen zur Trinkwassergewinnung betrieben. Standardmeßgerät ist bei beiden Meßnetzen der Hellmann-Regenmesser. Die Meßmethodik der DWD- und der HWW-Stationen ist absolut vergleichbar und drückt sich auch durch die Aufnahme einiger HWW-Stationen in das Meßnetz des DWD aus. Diese Stationen werden in der vorliegenden Untersuchung als DWDStationen verwendet. Bei einer ausreichend langen Beobachtungsperiode von mindestens 25 Jahren im Untersuchungszeitraum 1961 bis 1990 wurden die restlichen Stationen als HWW-Stationen geführt.

Durch die erhebliche Verdichtung des DWD-Niederschlagmeßnetzes gegenüber dem Temperaturmeßnetz durch von Laien betreute Stationen (Niederschlagmeßstationen) ist die geographische Verteilung aller hier zur Verfügung stehenden Stationen über ganz Niedersachsen als repräsentativ anzusehen. Einen Überblick gibt Abbildung 17. 
Abbildung 17: Räumliche Verteilung der DWD-Niederschlagstationen (Klimahauptstationen, Wetterstationen und Niederschlagmeßstationen) und der Meßstationen der Harzwasserwerke (HWW) in Niedersachsen

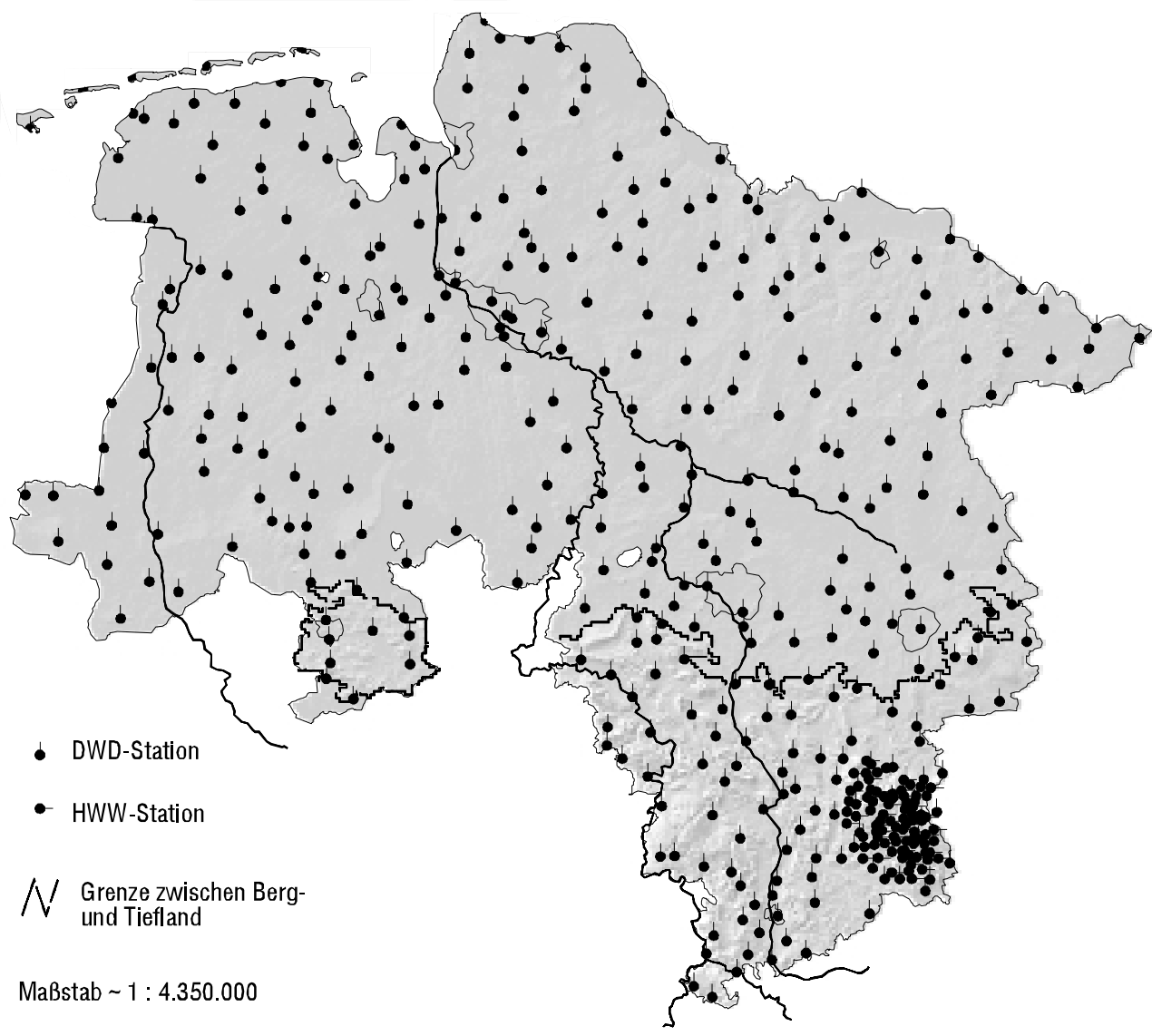

Die Lage der Niederschlagstationen im Harz wird wegen der Meßnetzverdichtung durch die Integration der HWW-Stationen auch gesondert in Abbildung 18 dargestellt. 
Abbildung 18: Räumliche Verteilung der DWD-Niederschlagstationen (Klimahauptstationen, Wetterstationen und Niederschlagmeßstationen) und der Meßstationen der Harzwasserwerke (HWW) im niedersächsischen Harz

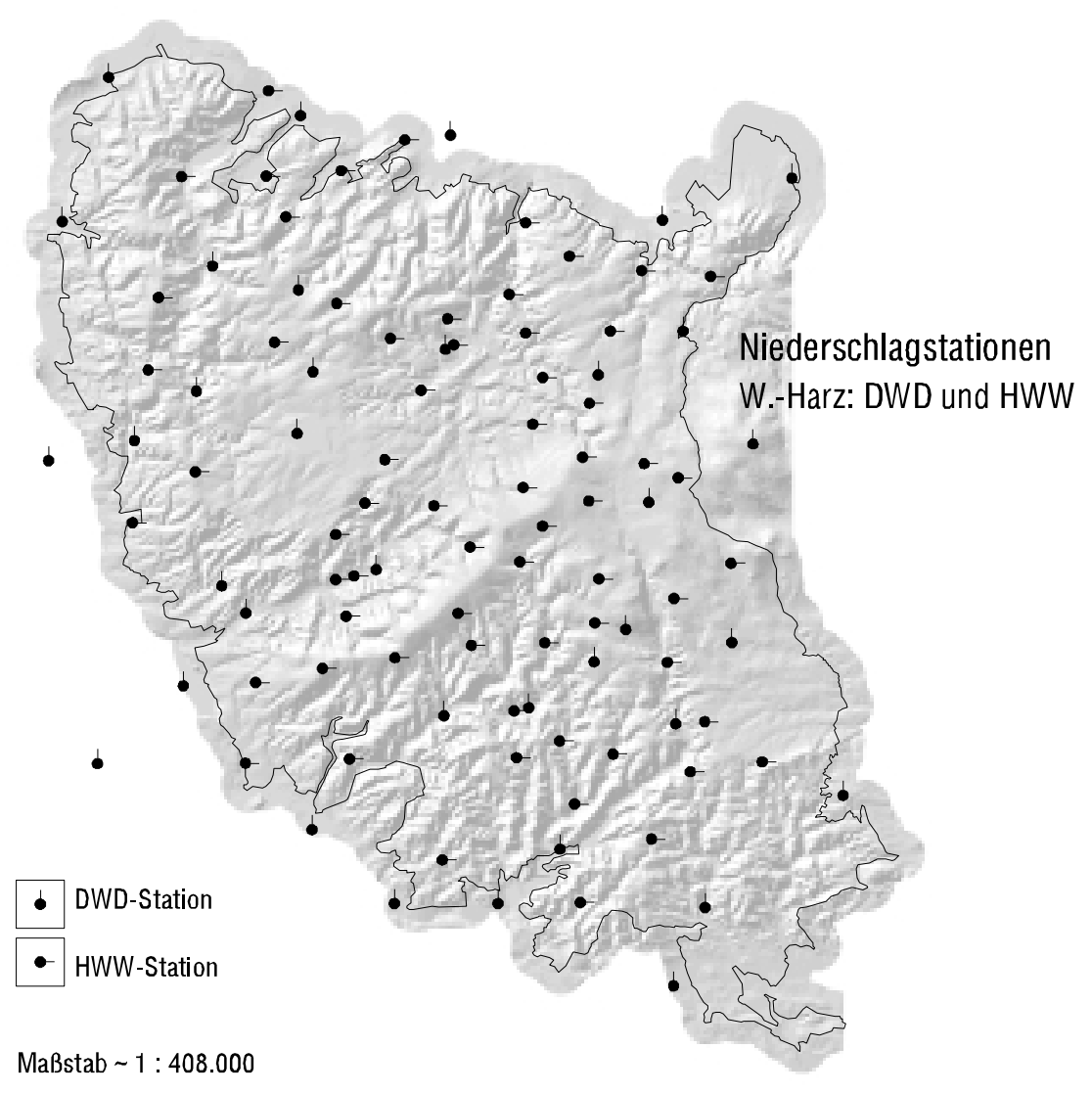

Vor allem durch Windeinwirkung entstehen bei Verwendung des HellmannRegenmessers Meßfehler, die zu z.T. erheblichen Unterschätzungen des Niederschlags führen. KLEIN (1994, S. 10) gibt mit Verweis auf KURTYKA (1953) die in Tabelle 21 dargestellten Fehlerrahmen an. Von KUMm (1983) und RodDA (1971) werden teilweise noch erheblich höhere Fehler durch Windeinfluß beschrieben. RoDDA (1971) führt im Sommer allerdings die größten Verluste auf Verdunstung und Benetzungsverluste zurück.

Tabelle 21: Fehlerrahmen für Niederschlagsmessungen nach KURTYKA (1953) aus KLEIN (1994)

\begin{tabular}{lc}
\hline Fehlerquelle & Fehlerrahmen \\
\hline Verdunstung & $-1 \%$ \\
Adhäsion & $-0,5 \%$ \\
Gerätefarbe & $-0,5 \%$ \\
Neigung der Öffnung & $-0,5 \%$ \\
Spritzer & $-0,5 \%$ \\
Exposition des Gerätes (Windfelddeformationen) & -5 bis - $80 \%$ \\
\hline
\end{tabular}


Analog zu der Regionalisierung der Lufttemperatur wurde auch für den Niederschlag eine Trennung zwischen Bergland und Tiefland durchgeführt. Ebenfalls getrennt soll daher die Untersuchung der Repräsentanz erfolgen. Daten von 128 Stationen des DWD und 65 der HWW stehen für die Regionalisierung der Niederschlagssummen im Bergland zur Verfügung, 260 Stationen des DWD lieferten die für die Regionalisierung im Tiefland verwendeten Daten. Um einen Gesamteindruck der Niederschlagsverhältnisse in Niedersachsen $\mathrm{zu}$ geben, sollen vor der Repräsentanzuntersuchung die vom DWD gemessenen Niederschlagssummen in Tabelle 22 in Form von deskriptiven Statistiken für ganz Niedersachsen dargestellt werden. Die HWW-Stationen wurden hier nicht berücksichtigt, um eine Überrepräsentanz des Harzes zu vermeiden (vgl. Kap. 2.1).

Tabelle 22: Deskriptive Statistiken zu den durchschnittlichen Niederschlägen im Gesamtdatenkollektiv für Niedersachsen des DWD

\begin{tabular}{lccccr}
\hline Monat/Jahr & n obs & $\begin{array}{c}\text { Min. } \\
{[\mathbf{1} / \mathbf{1 0} \mathbf{~ m m}]}\end{array}$ & $\begin{array}{c}\text { Max. } \\
{[\mathbf{1} / \mathbf{1 0} \mathbf{~ m m}]}\end{array}$ & $\begin{array}{c}\text { Mittelw. } \\
{[\mathbf{1} / \mathbf{1 0} \mathbf{~ m m}]}\end{array}$ & $\begin{array}{c}\text { Stdabw. } \\
{[\mathbf{1 / 1 0} \mathbf{~ m m}]}\end{array}$ \\
\hline Jan & 388 & 350 & 1820 & 643,06 & 179,92 \\
Feb & 388 & 307 & 1334 & 462,12 & 130,96 \\
Mär & 388 & 361 & 1594 & 567,79 & 147,80 \\
Apr & 388 & 390 & 1264 & 540,59 & 113,91 \\
Mai & 388 & 468 & 1140 & 629,29 & 97,04 \\
Jun & 388 & 610 & 1419 & 787,21 & 119,92 \\
Jul & 388 & 541 & 1304 & 740,07 & 114,55 \\
Aug & 388 & 543 & 1308 & 715,88 & 96,68 \\
Sep & 388 & 380 & 1227 & 609,26 & 115,93 \\
Okt & 388 & 340 & 1334 & 568,87 & 139,97 \\
Nov & 388 & 417 & 1820 & 684,64 & 174,19 \\
Dez & 388 & 458 & 2112 & 731,91 & 213,19 \\
\hline Jahr & 388 & 5452 & 17676 & 7680,60 & 1518,37 \\
\hline
\end{tabular}

Die in Tabelle 22 beschriebenen Verteilungen der gemessenen monatlichen und jährlichen Niederschlagssummen zeigen saisonale Effekte. Zwei lokale Maxima sind festzustellen, von denen eines im Winter (73,2 $\mathrm{mm}$ im Dezember), das andere im Sommer liegt $(78,7 \mathrm{~mm}$ im Juni). Die Streuung der Meßwerte (hier: Standardabweichungen) ist im Winter grundsätzlich höher als im Sommer. Dies ist auch gerade im Verhältnis zu den Mittelwerten zu beobachten. Die Extremwerte zeigen erhebliche Unterschiede mit einem maximalen Wertebereich (= max $-\min )$ im Dezember von 165,4 mm! 


\subsubsection{Niederschlagssumme im Bergland}

Die Niederschlagsverhältnisse im Bergland können zunächst über deskriptive Statistiken der Meßwerte der Datenkollektive beschrieben werden (Tabelle 23). Die hohen Niederschläge in den Wintermonaten sind durch verstärkten Schneefall in diesem Gebiet zu erklären. Die Standardabweichung der gemessenen Werte ist von November bis März am höchsten, was auf unterschiedliche Meßsituation der Stationen besonders in Hinblick auf Schneefallereignisse hinweist.

Tabelle 23: Deskriptive Statistiken zu den durchschnittlichen Niederschlägen im Berglanddatenkollektiv des DWD mit 128 Stationen

\begin{tabular}{lccccc}
\hline Monat/Jahr & n obs & $\begin{array}{c}\text { Min. } \\
{[\mathbf{1} / \mathbf{1 0} \mathbf{~ m m}]}\end{array}$ & $\begin{array}{c}\text { Max. } \\
{[\mathbf{1} / \mathbf{1 0} \mathbf{~ m m}]}\end{array}$ & $\begin{array}{c}\text { Mittelw. } \\
{[\mathbf{1} / \mathbf{1 0} \mathbf{~ m m}]}\end{array}$ & $\begin{array}{c}\text { Stdabw. } \\
{[\mathbf{1 / 1 0} \mathbf{~ m m}]}\end{array}$ \\
\hline Jan & 128 & 350 & 1820 & 701,54 & 285,77 \\
Feb & 128 & 329 & 1334 & 536,20 & 197,59 \\
Mär & 128 & 404 & 1594 & 635,40 & 228,34 \\
Apr & 128 & 436 & 1264 & 629,35 & 156,79 \\
Mai & 128 & 500 & 1140 & 705,87 & 121,64 \\
Jun & 128 & 659 & 1419 & 877,05 & 161,55 \\
Jul & 128 & 541 & 1304 & 766,49 & 167,12 \\
Aug & 128 & 594 & 1308 & 754,21 & 143,38 \\
Sep & 128 & 380 & 1227 & 601,56 & 153,37 \\
Okt & 128 & 343 & 1334 & 551,51 & 184,44 \\
Nov & 128 & 417 & 1820 & 706,05 & 256,49 \\
Dez & 128 & 460 & 2112 & 831,83 & 329,32 \\
\hline Jahr & 128 & 5625 & 17676 & 8296,97 & 2338,88 \\
\hline
\end{tabular}

Die Statistiken für die HWW-Stationen bestätigen die für die DWD-Stationen gemachten Aussagen (Tabelle 24). Die durchschnittlichen Niederschlagssummen liegen über denen des DWD-Datenkollektivs, was durch die Lage der Stationen im Harz im Gegensatz zur relativ gleichmäßigen Verteilung der DWD-Stationen über das gesamte niedersächsische Bergland erklärt werden kann. 
Tabelle 24: Deskriptive Statistiken zu den durchschnittlichen Niederschlägen im Datenkollektiv der Harzwasserwerke (HWW)

\begin{tabular}{lcccrc}
\hline Monat/Jahr & n obs & $\begin{array}{c}\text { Min. } \\
{[\mathbf{1} / \mathbf{1 0} \mathbf{~ m m}]}\end{array}$ & $\begin{array}{c}\text { Max. } \\
{[\mathbf{1} / \mathbf{1 0} \mathbf{~ m m}]}\end{array}$ & $\begin{array}{c}\text { Mittelw. } \\
{[\mathbf{1} / \mathbf{1 0} \mathbf{~ m m}]}\end{array}$ & $\begin{array}{c}\text { Stdabw. } \\
{[\mathbf{1} / \mathbf{1 0} \mathbf{~ m m}]}\end{array}$ \\
\hline Jan & 65 & 670 & 1650 & 1158,15 & 251,25 \\
Feb & 65 & 490 & 1210 & 863,69 & 172,70 \\
Mär & 65 & 640 & 1400 & 1018,77 & 195,41 \\
Apr & 65 & 630 & 1160 & 903,54 & 130,16 \\
Mai & 65 & 600 & 1100 & 886,62 & 110,97 \\
Jun & 65 & 790 & 1350 & 1114,00 & 136,44 \\
Jul & 65 & 640 & 1340 & 1044,46 & 159,92 \\
Aug & 65 & 650 & 1210 & 970,46 & 115,98 \\
Sep & 65 & 520 & 1100 & 847,38 & 149,56 \\
Okt & 65 & 530 & 1140 & 858,31 & 164,67 \\
Nov & 65 & 640 & 1560 & 1118,77 & 239,63 \\
Dez & 65 & 780 & 1980 & 1396,62 & 311,22 \\
\hline Jahr & 65 & 7630 & 15950 & 12181,08 & 2047,40 \\
\hline
\end{tabular}

Die in Tabelle 25 zusammengestellten deskriptiven Statistiken zu den Verteilungen von sechs Variablen für die DWD Niederschlagstationen im Bergland und ein Raster mit $50 \mathrm{~m}$ Rasterweite zeigen im Vergleich zu den Verteilungen der Temperaturstationen bis auf die Verteilung der Variable Kaltluftabflu $\beta$ stets einen größeren Wertebereich (vgl. Tabelle 15, Seite 64).

Tabelle 25: Gegenüberstellung von Variablenverteilungen für das Bergland und 128 DWD Niederschlagmeßstationen im Bergland

\begin{tabular}{|c|c|c|c|c|c|c|c|c|}
\hline \multicolumn{4}{|c|}{$\begin{array}{c}\text { Bergland } \\
\text { (Rasterwerte; Rasterweite: } \mathbf{5 0} \mathbf{~ m} \text { ) }\end{array}$} & \multirow[t]{2}{*}{ Variable } & \multicolumn{4}{|c|}{$\begin{array}{l}\text { DWD Niederschlagstationen } \\
\text { Bergland }(n=128)\end{array}$} \\
\hline Min. & Max. & Mittelw. & Stdabw. & & Min. & Max. & Mittelw. & Stdabw. \\
\hline 35 & 953 & 218,23 & 130,75 & $\begin{array}{l}\text { Geländehöhe } \\
\text { [m ü. NN] }\end{array}$ & 60 & $1132^{3}$ & 225,86 & 170,14 \\
\hline 0 & 136 & 8,34 & 8,97 & $\begin{array}{c}\text { Hangneigung } \\
{[\%]}\end{array}$ & 0 & 43 & 6,71 & 7,28 \\
\hline 0 & 368 & 107,61 & 33,91 & $\begin{array}{c}\text { Insolation Januar } \\
{[\text { ] }}\end{array}$ & 0 & 254 & 110,09 & 28,34 \\
\hline 0 & 36 & 33,25 & 5,50 & $\begin{array}{l}\text { Rel. Exponiertheit } \\
\text { (0 bis } 36)\end{array}$ & 1 & 36 & 33,04 & 6,99 \\
\hline 0 & $\begin{array}{r}200 \\
0\end{array}$ & 92,25 & 311,76 & $\begin{array}{l}\text { Kaltluftabfluß } \\
(0 \text { bis } 2000)\end{array}$ & 1 & 2000 & 184,23 & 410,46 \\
\hline 0 & $\begin{array}{r}109 \\
6\end{array}$ & 110,10 & 110,67 & $\begin{array}{l}\text { Leewirbel } \\
{[\mathrm{m}]}\end{array}$ & 0 & 595 & 128,97 & 130,25 \\
\hline
\end{tabular}

Die Maxima der Variablen Hangneigung und Leewirbel sind mit $43 \%$ bzw. einem Wert von $595 \mathrm{~m}$ im Vergleich zu den Maxima der entsprechenden

3. Meßstation „Brocken“ befindet sich nicht in Niedersachsen aber in direkter Nachbarschaft 
Temperaturstationsverteilungen mehr als doppelt so groß. Auch die Variable Insolation im Januar hat einen größeren Wertebereich (0 bis 254 statt 63 bis 162). Die Variable Relative Exponiertheit zeigt einen Wertebereich von 1 bis 36. Es wird damit fast das gesamte mögliche Spektrum $(0$ bis 36$)$ durch die Niederschlagstationen des DWD erfaßt. Auch das Minimum der Variable Geländehöhe liegt unter dem der Temperaturstationsverteilung $(60 \mathrm{~m}$ ü. NN statt $72 \mathrm{~m}$ ü. NN). Es kann somit im Vergleich mit der Temperaturregionalisierung von einer besseren Repräsentanz des Zielgebietes durch die Stationen ausgegangen werden. Durch die Hinzunahme der HWW-Stationen kann diese Repräsentanz noch weiter gesteigert werden, wie aus Tabelle 26 hervorgeht.

Tabelle 26: Gegenüberstellung von Variablenverteilungen (deskriptive Statistiken) für den Harz und entsprechende HWW Niederschlagstationen und die Verteilung des gemeinsamen DWD/HWW Datenkollektivs (hier: nur Harzstationen)

\begin{tabular}{|c|c|c|c|c|c|c|c|c|}
\hline \multicolumn{4}{|c|}{ Harz } & \multirow[t]{2}{*}{ Variable } & \multicolumn{4}{|c|}{$\begin{array}{c}\text { Niederschlagstationen im Harz } \\
\text { DWD/HWW / nur HWW (n=103 / 65) }\end{array}$} \\
\hline Min. & Max. & Mittelw. & Stdabw. & & Min. & Max. & Mittelw. & Stdabw. \\
\hline \multirow[t]{2}{*}{161} & 953 & 479,73 & 153,30 & Geländehöhe & 192 & $1132^{4}$ & 496,33 & 187,57 \\
\hline & & & & [m ü. NN] & 203 & 876 & 534,87 & 164,34 \\
\hline \multirow[t]{2}{*}{0} & 136 & 18,26 & 13,90 & Hangneigung & 1 & 69 & 15,43 & 12,98 \\
\hline & & & & {$[\%]$} & 1 & 69 & 17,88 & 14,31 \\
\hline \multirow[t]{2}{*}{0} & 368 & 106,10 & 56,30 & Insolation Jan. & 0 & 254 & 105,89 & 46,26 \\
\hline & & & & [ ] & 0 & 229 & 105,13 & 48,29 \\
\hline \multirow[t]{2}{*}{0} & 36 & 27,15 & 9,03 & Rel. Exponiertheit & 1 & 36 & 25,74 & 10,51 \\
\hline & & & & (0 bis 36$)$ & 4 & 36 & 24,71 & 10,23 \\
\hline \multirow[t]{2}{*}{0} & 2000 & 81,67 & 277,86 & Kaltluftabfluß & 1 & 2000 & 222,15 & 463,73 \\
\hline & & & & (0 bis 2000$)$ & 1 & 2000 & 139,80 & 386,04 \\
\hline \multirow[t]{2}{*}{0} & 1096 & 178,22 & 167,44 & Leewirbel & 0 & 714 & 233,23 & 199,06 \\
\hline & & & & [m] & 0 & 714 & 250,12 & 215,78 \\
\hline
\end{tabular}

Verbesserungen der Repräsentanz zeigen sich vor allem durch die Ausweitung des Wertebereichs der Variable Hangneigung bis auf $69 \%$ (!) und die Erhöhung des Maximums der Variable Leewirbel auf $714 \mathrm{~m}$. Durch Stationen des HWW wird das Datenkollektiv folglich um Orte mit vorgelagerten Bergen und an steilen Hängen erweitert. Abbildung 19 und Abbildung 20 veranschaulichen, daß für mehrere Stationen Werte für Leewirbel und Hangneigung über den Maxima der DWDStationen ermittelt wurden.

4. Meßstation „Brocken“ befindet sich nicht in Niedersachsen aber in direkter Nachbarschaft 
Abbildung 19: Verteilung der Variable Leewirbel der Niederschlagmeßstationen des DWD/HWW-Datenkollektivs im Harz mit derjenigen für den gesamten niedersächsischen Harz, vertreten durch die Zellen eines digitalen Höhenmodells (DHM) mit Rasterweite 50 m

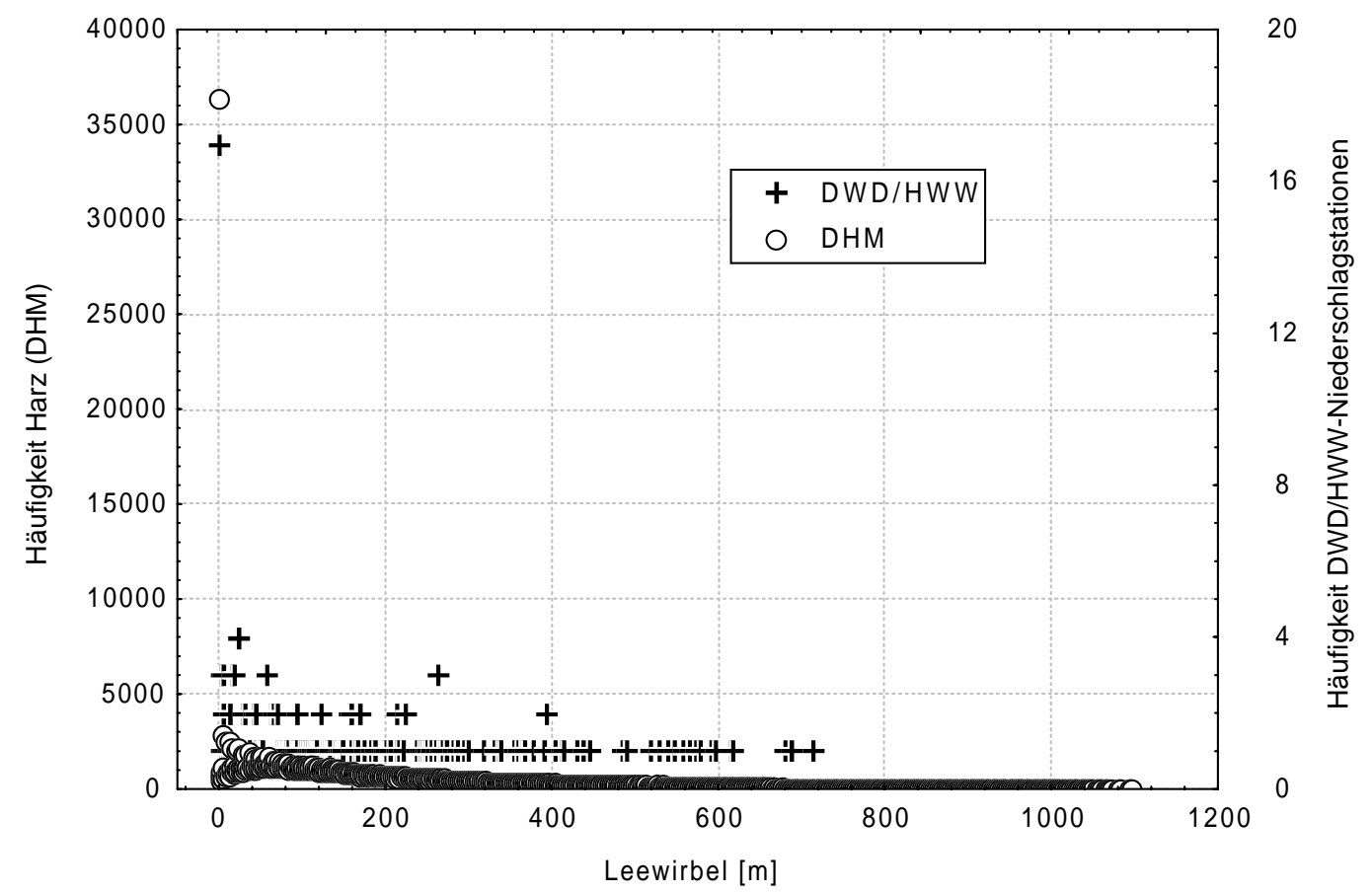

Abbildung 20: Verteilung der Variable Hangneigung der Niederschlagstationen des DWD/HWW-Datenkollektivs im Harz zusammen mit derjenigen für den gesamten niedersächsischen Harz (Rasterweite: 50 m)

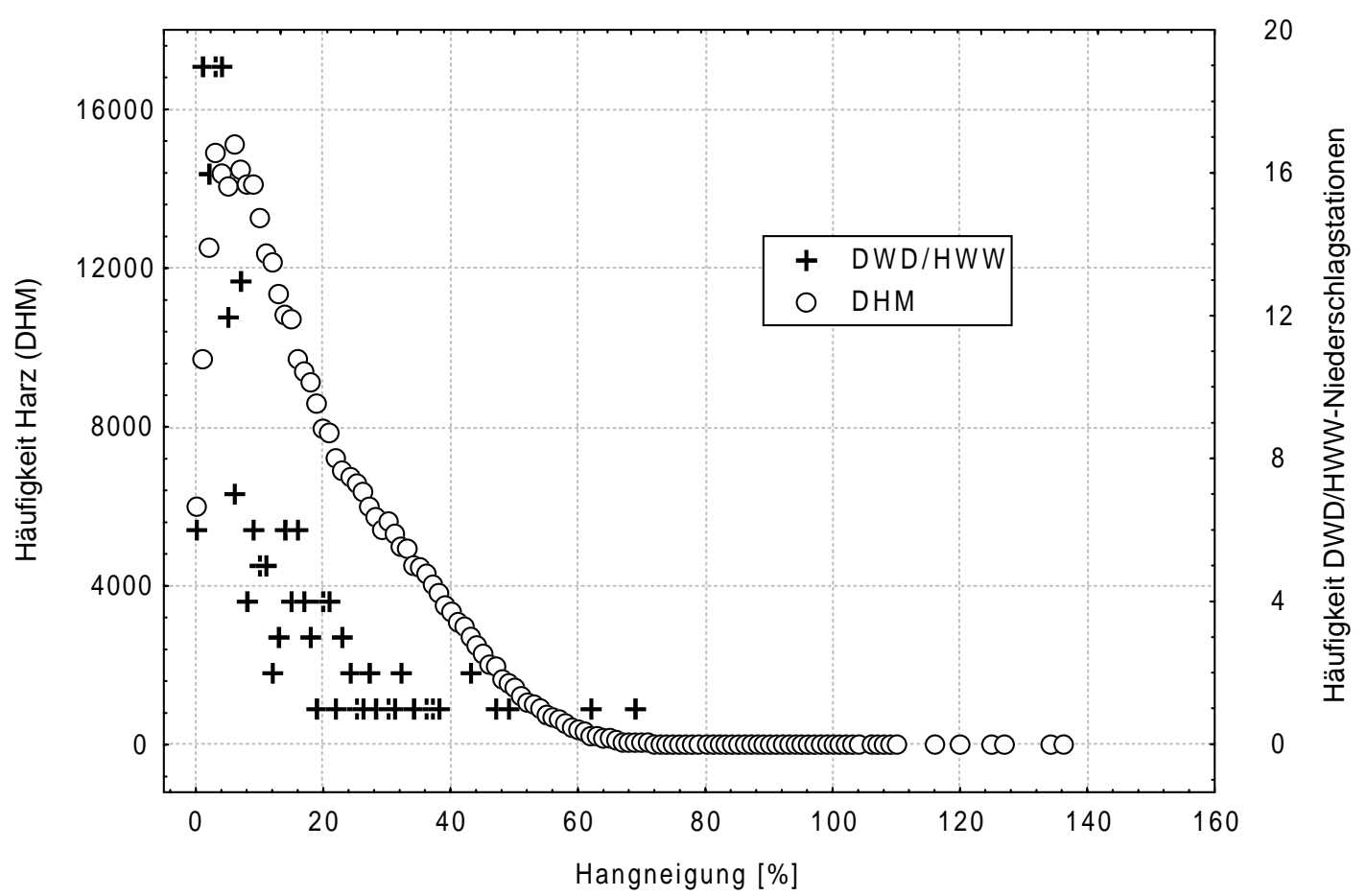


Daß die nicht durch das gemeinsame Datenkollektiv repräsentierten Teile des Zielgebietes von vernachlässigbarer Größe sind, geht aus Tabelle 27 hervor. Lediglich für die Variable Kaltluftabfluß wird ein flächenmäßig beachtenswerter Repräsentanzmangel festgestellt, der jedoch im Wertebereich der Variable völlig unbedeutend ist, da nur das mögliche Minimum 0 nicht von den Stationen realisiert wird.

Tabelle 27: Quantifizierung der Repräsentanzmängel der DWD/HWWNiederschlagmeßstationen im Bergland in Form der prozentualen Angabe von Rasterwerten, die nicht durch das Spektrum der Meßstationswerte abgedeckt werden

\begin{tabular}{|c|c|}
\hline Variable & Repräsentanzmangel \\
\hline $\begin{array}{l}\text { Geländehöhe } \\
\text { [m ü. NN] }\end{array}$ & $0,6 \%$ unter min. der Stationswerte \\
\hline $\begin{array}{c}\text { Hangneigung } \\
{[\%]}\end{array}$ & $0,02 \%$ über max. der Stationswerte \\
\hline $\begin{array}{l}\text { Insolation Jan. } \\
\text { [ ] }\end{array}$ & $\begin{array}{l}0,1 \% \text { außerhalb des Bereichs der Stationswerte; } \\
\text { Extremwerte unterscheiden sich stark }\end{array}$ \\
\hline $\begin{array}{l}\text { Rel. Exponiertheit } \\
\text { (0 bis 36) }\end{array}$ & $0,01 \%$ unter min. der Stationswerte \\
\hline $\begin{array}{l}\text { Kaltluftabfluß } \\
(0 \text { bis } 2000)\end{array}$ & $8,8 \%$ unter min. der Stationswerte (Wert: 0 ) \\
\hline $\begin{array}{l}\text { Leewirbel } \\
{[\mathrm{m}]}\end{array}$ & $\begin{array}{l}0,07 \% \text { über max. der Stationswerte; Maxima } \\
\text { unterscheiden sich stark (714 gegen 1096) }\end{array}$ \\
\hline
\end{tabular}

\subsubsection{Niederschlagssumme im Tiefland}

Tabelle 28 kann entnommen werden, daß nur in den Monaten September und Oktober die Niederschläge im niedersächsischen Tiefland durchschnittlich höher sind als im Bergland (vgl. Tabelle 23, S. 76). Die Standardabweichung der Tieflanddaten hat viel geringere Werte als für das Bergland ermittelt.

Tabelle 28: Deskriptive Statistiken zu den durchschnittlichen Niederschlägen im Tieflanddatenkollektiv des DWD

\begin{tabular}{lccccr}
\hline Monat/Jahr & n obs & $\begin{array}{c}\text { Min. } \\
{[\mathbf{1 / 1 0} \text { mm }]}\end{array}$ & $\begin{array}{c}\text { Max. } \\
{[\mathbf{1 / 1 0} \text { mm }]}\end{array}$ & $\begin{array}{c}\text { Mittelw. } \\
{[\mathbf{1} / \mathbf{1 0} \mathbf{~ m m}]}\end{array}$ & $\begin{array}{c}\text { Stdabw. } \\
{[\mathbf{1} / \mathbf{1 0} \mathbf{~ m m}]}\end{array}$ \\
\hline Jan & 260 & 414 & 924 & 614,28 & 76,19 \\
Feb & 260 & 307 & 653 & 425,65 & 49,36 \\
Mär & 260 & 361 & 827 & 534,51 & 60,87 \\
Apr & 260 & 390 & 682 & 496,89 & 39,05 \\
Mai & 260 & 468 & 805 & 591,60 & 49,90 \\
Jun & 260 & 610 & 927 & 742,97 & 52,30 \\
Jul & 260 & 551 & 941 & 727,06 & 73,45 \\
Aug & 260 & 543 & 843 & 697,00 & 52,93 \\
Sep & 260 & 413 & 858 & 613,05 & 92,21 \\
Okt & 260 & 340 & 860 & 577,42 & 111,22 \\
Nov & 260 & 425 & 964 & 674,11 & 112,88 \\
Dez & 260 & 458 & 1020 & 682,72 & 85,84 \\
\hline Jahr & 260 & 5452 & 9864 & 7377,16 & 694,45 \\
\hline
\end{tabular}


Analog zu den Ergebnissen der Repräsentanzüberprüfungen für das Bergland kann auch für das Tiefland gegenüber dem Temperaturmeßnetz eine Verbesserung der Repräsentanz durch die höhere Dichte des Niederschlagmeßnetzes festgestellt werden.

Tabelle 29: Gegenüberstellung von Variablenverteilungen (deskriptive Statistiken) für das Tiefland und entsprechende DWDNiederschlagstationen

\begin{tabular}{|c|c|c|c|c|c|c|c|c|}
\hline \multicolumn{4}{|c|}{$\begin{array}{c}\text { Tiefland } \\
\text { (Rasterwerte; Rasterweite: } 50 \mathrm{~m} \text { ) }\end{array}$} & Variable & \multicolumn{4}{|c|}{$\begin{array}{l}\text { DWD Niederschlagstationen } \\
\text { im Tiefland }(n=260)\end{array}$} \\
\hline $\begin{array}{l}\text { Min. } \\
-11\end{array}$ & $\begin{array}{l}\text { Max. } \\
172\end{array}$ & $\begin{array}{l}\text { Mittelw. } \\
35,53\end{array}$ & $\begin{array}{r}\text { Stdabw. } \\
28,35\end{array}$ & Geländehöhe & $\begin{array}{c}\text { Min. } \\
0\end{array}$ & $\begin{array}{l}\text { Max. } \\
131\end{array}$ & $\begin{array}{c}\text { Mittelw. } \\
32,28\end{array}$ & $\begin{array}{r}\text { Stdabw. } \\
28,40\end{array}$ \\
\hline 0 & 63 & 0,68 & 1,34 & $\begin{array}{c}\text { Hangneigung } \\
{[\%]}\end{array}$ & 0 & 10 & 1,26 & 1,70 \\
\hline 0 & 306 & 108,95 & 5,06 & $\begin{array}{c}\text { Insolation Jan. } \\
\text { [ ] }\end{array}$ & 70 & 143 & 109,71 & 6,39 \\
\hline 0 & 36 & 35,99 & 0,28 & $\begin{array}{l}\text { Rel. Exponiertheit } \\
\quad(0 \text { bis } 36)\end{array}$ & 35 & 36 & 36,00 & 0,05 \\
\hline 0 & 2000 & 138,01 & 398,09 & $\begin{array}{l}\text { Kaltluftabfluß } \\
(0 \text { bis } 2000)\end{array}$ & 0 & $\begin{array}{r}200 \\
0\end{array}$ & 148,88 & 448,01 \\
\hline 0 & 406 & 12,04 & 18,74 & $\begin{array}{l}\text { Leewirbel } \\
{[\mathrm{m}]}\end{array}$ & 0 & 213 & 17,93 & 30,63 \\
\hline
\end{tabular}

Die Werte der deskriptiven Statistiken zu den Verteilungen der Raster- und Stationswerte exemplarischer räumlicher Variablen (Tabelle 29) weisen trotz der höheren Meßnetzdichte immer noch Lücken der Repräsentanz auf. Vor allem die Maxima der Hangneigung, der Insolation im Januar oder der Variable Leewirbel an den Stationen lassen große Unterschiede zu den Maxima der Raster erkennen. Diese Lücken sind allerdings flächenmäßig vernachlässigbar gering, wie Tabelle 30 entnommen werden kann.

Tabelle 30: Quantifizierung der Repräsentanzmängel der DWD-Niederschlagmeßstationen im Tiefland in Form der prozentualen Angabe von Rasterwerten, die nicht durch das Spektrum der Meßstationswerte abgedeckt werden

\begin{tabular}{cl}
\hline Variable & Repräsentanzmangel \\
\hline $\begin{array}{c}\text { Geländehöhe } \\
\text { [m ü. NN] }\end{array}$ & $0,4 \%$ drunter; $0,02 \%$ drüber \\
$\begin{array}{c}\text { Hangneigung } \\
{[\%]}\end{array}$ & $0,16 \%$ drüber \\
$\begin{array}{c}\text { Insolation Jan. } \\
{[]}\end{array}$ & $0,07 \%$ drunter; $0,10 \%$ drüber \\
$\begin{array}{c}\text { Rel. Exponiertheit } \\
\text { (0 bis 36) } \\
\text { Kaltluftabfluß } \\
(0 \text { bis } 2000) \\
\text { Leewirbel } \\
{[\mathrm{m}]}\end{array}$ & Nur $0,14 \%$ drunter ! \\
\hline
\end{tabular}


Es kann somit von nahezu uneingeschränkter Repräsentanz des Niedersächsischen Tieflandes durch die DWD-Niederschlagmeßstationen gesprochen werden. Die durch den Vergleich der Verteilungsextrema gezeigten Einschränkungen für Variablen, die in die Modelle integriert sind, müssen jedoch bei der Regionalisierung und bei der Umsetzung der Regionalisierungsergebnisse Berücksichtigung finden. Dies geschah im Rahmen dieser Arbeit, wie es auf Seite 66 im Zusammenhang mit den Temperaturmeßstationen des DWD im Bergland beschrieben wurde, durch „Kappung“.

\subsubsection{Relative Luftfeuchte}

Die Relative Luftfeuchte ist definiert als das Verhältnis aus der spezifischen Luftfeuchte und der Sättigungsfeuchte der Luft. Die Sättigungsfeuchte S der Luft gibt an, wieviel g Wasserdampf maximal in $1 \mathrm{~kg}$ feuchter Luft enthalten sein können. Diese Größe ist temperaturabhängig. Kalte Luft kann wenig, warme Luft kann viel Wasserdampf enthalten. Der tatsächliche Wassergehalt der Luft ist die spezifische Feuchte, die ebenso wie die Sättigungsfeuchte in g Wasserdampf pro kg feuchte Luft gemessen wird (HÄCKEL, 1990, S. 43ff). Setzt man den tatsächlichen Wasserdampfgehalt der Luft, die spezifische Feuchte s, ins Verhältnis zur Sättigungsfeuchte S, so erhält man die relative Luftfeuchte RF:

$$
\mathrm{RF}=\frac{\mathrm{S}}{\mathrm{S}} * 100 \%
$$

Die Klimahauptstationen und die Wetterstationen des DWD erfassen standardmäßig neben der Lufttemperatur (vgl. Kap. 4.1.2) u. a. auch die relative Luftfeuchte. Es stehen hierzu ein trockenes und ein feuchtes Thermometer zur Verfügung, die zusammen das sogenannte Psychrometer bilden. Ein Aspirator sorgt für einen gleichmäßigen Luftstrom am feuchten Thermometer (vgl. DWD, 1980). Dieser Luftstrom sorgt an dem in Wasser getauchten feuchten Thermometer für eine Abkühlung durch Verdunstung. Je trockener die vorbeiströmende Luft ist, desto stärker muß die Abkühlung während einer konstanten Zeit sein. Dies führt zu einer Temperaturdifferenz zwischen trockenem und feuchtem Thermometer, der sogenannten Psychrometerdifferenz, die zur Berechnung der relativen Luftfeuchte dient (Ḧ̈CKEL, 1990, S. 332ff). 
Ebenso wie oben für Lufttemperatur und Niederschlagssummen beschrieben, wurde auch die relative Luftfeuchte getrennt für Berg- und Tiefland regionalisiert. Wie bereits für Lufttemperatur und Niederschlagssummen soll auch hier in den folgenden Unterkapiteln die Repräsentanz für die Untergebiete untersucht werden. Die Ergebnisse einer Untersuchung bezüglich möglicher Verbesserungen der Präzision der Modelle durch die getrennte Regionalisierung der relativen Luftfeuchte wird wie im Fall der Klimaelemente Lufttemperatur und Niederschlagssumme im Ergebnisteil dargestellt (Kap. 5.1.3).

\subsubsection{Relative Luftfeuchte im Bergland}

Die Mittelwerte der relativen Luftfeuchte im Berglanddatenkollektiv des DWD zeigen einen klaren jährlichen Trend (s. Tabelle 31). Während für die Wintermonate hohe relative Luftfeuchten gemessen wurden, liegen die Werte für die Sommermonate um ca. $5 \%$ tiefer. Die Standardabweichungen der monatlichen und jährlichen Meßwerte zeigen eine andere Saisonalität als Mittelwerte und Extrema. Die höchsten Standardabweichungen werden im Frühjahr beobachtet $(3,93 \%$ im März), die niedrigsten im Herbst (2,03 \% im Oktober).

Tabelle 31: Deskriptive Statistiken zur durchschnittlichen relativen Luftfeuchte im Berglanddatenkollektiv des DWD

\begin{tabular}{lccccc}
\hline Monat/Jahr & $\mathbf{n}$ & $\begin{array}{r}\text { Min. } \\
{[\mathbf{\%}]}\end{array}$ & $\begin{array}{c}\text { Max } \\
{[\mathbf{\%}]}\end{array}$ & $\begin{array}{c}\text { Mittelw. } \\
{[\%]}\end{array}$ & $\begin{array}{c}\text { Stdabw. } \\
{[\%]}\end{array}$ \\
\hline Jan & 15 & 81 & 90 & 85,53 & 2,47 \\
Feb & 15 & 80 & 88 & 83,27 & 2,40 \\
Mär & 15 & 74 & 90 & 80,20 & 3,93 \\
Apr & 15 & 71 & 87 & 76,00 & 3,84 \\
Mai & 15 & 70 & 83 & 73,60 & 3,38 \\
Jun & 15 & 71 & 85 & 75,33 & 3,31 \\
Jul & 15 & 71 & 86 & 76,20 & 3,47 \\
Aug & 15 & 72 & 85 & 76,53 & 3,11 \\
Sep & 15 & 76 & 89 & 81,47 & 2,95 \\
Okt & 15 & 79 & 87 & 83,40 & 2,03 \\
Nov & 15 & 80 & 90 & 85,60 & 2,75 \\
Dez & 15 & 82 & 91 & 86,67 & 2,35 \\
\hline Jahr & 15 & 76 & 87 & 80,20 & 2,78 \\
\hline
\end{tabular}

Im Vergleich zum Umfang des Temperaturdatenkollektivs für das niedersächsische Bergland gibt es nur einen Unterschied: Die Klimahauptstation „Einbeck“ lieferte zwar für den Beobachtungszeitraum (1961 bis 1990) einen verwendbaren Datensatz für die Lufttemperatur, nicht aber für die relative Luftfeuchte und bestätigt somit als Ausnahme die Regel, an allen Klimahauptstationen würde auch die relative 
Luftfeuchte gemessen. Die Beschreibung der Repräsentanz der verwendeten Stationen beschränkt sich daher auf die durch den Wegfall der Station Einbeck im Vergleich zum Temperaturdatenkollektiv Bergland entstandenen Änderungen.

Für die DWD-Station „Einbeck“ (Nr. 54637) wurde der Maximalwert für die Variable Kaltluftabfluß, der Wert 2000 ermittelt. Eine diesbezügliche Untersuchung des DWD-Temperaturdatenkollektivs Bergland zeigt, daß nur an der Station Einbeck der maximale Wert der Variable Kaltluftabfluß von 2000 erreicht wird. Die Stationen Seesen und Bad Sachsa können mit den Werten 464 bzw. 349 nicht die volle Repräsentanz des Datenkollektivs begründen. So muß dieser Repräsentanzmangel für das Berglanddatenkollektiv zur Luftfeuchte registriert werden. Für alle anderen Variablen zeigte die Station Einbeck keine extremen Werte, weshalb ansonsten hier die gleiche Zielgebietsrepräsentanz gegeben ist wie für die Temperaturmeßstationen des DWD (Kap. 4.1.2.1).

\subsubsection{Relative Luftfeuchte im Tiefland}

Tabelle 32 zeigt deskriptive Statistiken zur durchschnittlichen relativen Luftfeuchte im Tieflanddatenkollektiv des DWD. Der für das Bergland festgestellte jährliche Trend der Mittelwerte zeigt sich ähnlich bei den Tieflandstationen. Dies sogar mit einer Amplitude von $14 \%$ (Dezember: $88 \%$, Mai: $74 \%$ ). Die Standardabweichungen zeigen keinen so klaren saisonalen Verlauf wie die Mittelwerte (zwei lokale Minima im Januar und im Oktober, Maximum im Mai), die Standardabweichungen sind stets geringer als für die Berglandstationen berechnet.

Tabelle 32: Deskriptive Statistiken zur durchschnittlichen relativen Luftfeuchte im Tieflanddatenkollektiv des DWD

\begin{tabular}{lccccc}
\hline Monat/Jahr & n & $\begin{array}{c}\text { Min. } \\
{[\%]}\end{array}$ & $\begin{array}{c}\text { Max } \\
{[\%]}\end{array}$ & $\begin{array}{c}\text { Mittelw. } \\
{[\mathbf{\%}]}\end{array}$ & $\begin{array}{c}\text { Stdabw. } \\
{[\%]}\end{array}$ \\
\hline Jan & 29 & 85 & 90 & 87,38 & 1,29 \\
Feb & 29 & 81 & 87 & 84,31 & 1,54 \\
Mär & 29 & 78 & 86 & 81,17 & 2,04 \\
Apr & 29 & 73 & 83 & 76,90 & 2,68 \\
Mai & 29 & 70 & 81 & 74,03 & 3,08 \\
Jun & 29 & 72 & 81 & 75,38 & 2,61 \\
Jul & 29 & 74 & 82 & 77,07 & 2,30 \\
Aug & 29 & 74 & 82 & 77,72 & 2,17 \\
Sep & 29 & 80 & 85 & 81,72 & 1,44 \\
Okt & 29 & 83 & 88 & 84,76 & 1,24 \\
Nov & 29 & 85 & 90 & 86,90 & 1,35 \\
Dez & 29 & 86 & 91 & 88,07 & 1,33 \\
\hline Jahr & 29 & 79 & 85 & 81,28 & 1,73 \\
\hline
\end{tabular}


Die Stationen des DWD, deren Daten zur Regionalisierung der relativen Luftfeuchte verwendet werden konnten, entsprechen denen des Temperaturdatenkollektivs Tiefland. Auf eine gesonderte Darstellung der Repräsentanz wird daher verzichtet und auf Kapitel 4.1.2.2, Seite 69ff verwiesen.

\subsection{Depositionsdaten}

Für den Meßzeitraum 1986 bis 1990 konnten von einer ausreichend großen Zahl von Meßstationen mittlere jährliche Einträge verschiedener Elemente berechnet werden. Die hierzu verwendeten Daten wurden zum größten Teil vom Niedersächsischen Landesamt für Ökologie (NLÖ) zur Verfügung gestellt. Dank für die gute Zusammenarbeit sei an dieser Stelle an Frau Gertsmeier und an Herrn Dr. Giesen gerichtet. Weitere Meßergebnisse lagen von verschiedenen Stationen des Forschungszentrums für Waldökosysteme (FZW) vor. Eine Zusammenstellung der verwendeten Stationen ist Anhang 3 zu entnehmen.

MEESEnBURG et al. (1994) stützen sich in ihrer Arbeit über die „Entwicklung der atmogenen Stoffeinträge in niedersächsische Waldbestände“ auf Meßwerte von Waldstationen, die auch mit anderem zeitlichem Rahmen für die vorliegende Untersuchung verwendet wurden. Sie beschreiben vor allem für Sulfat-Schwefel und Protonen einen Rückgang der Einträge von 1983 bis 1992. Auf eine erneute Darstellung des zeitlichen Trends der zur Verfügung stehenden Daten wird hier verzichtet. Vielmehr soll die räumliche Auswertung der Daten, die Regionalisierung für das Zielgebiet Niedersachsen beschrieben werden. Sie kann beispielsweise zur Verbesserung der Datengrundlage für die Anwendung eines wissensbasierten Systems zur Kalkungsplanung beitragen, wie es von JANSEN et al. (1994) beschrieben wurde.

In diesem Kapitel soll zunächst kurz auf die Prozesse eingegangen werden, die zur Deposition der verschiedenen Elemente führen (Kap. 4.2.1). Das Wissen um diese nutzte ULRICH (1991) zur Schätzung der Gesamtdepositionen in Wäldern. Daraufhin werden die verwendeten Datenkollektive vorgestellt. Ein deskriptiver Überblick und die Überprüfung der Repräsentanz der verwendeten Datenkollektive folgen, bevor 
Die Stationen des DWD, deren Daten zur Regionalisierung der relativen Luftfeuchte verwendet werden konnten, entsprechen denen des Temperaturdatenkollektivs Tiefland. Auf eine gesonderte Darstellung der Repräsentanz wird daher verzichtet und auf Kapitel 4.1.2.2, Seite 69ff verwiesen.

\subsection{Depositionsdaten}

Für den Meßzeitraum 1986 bis 1990 konnten von einer ausreichend großen Zahl von Meßstationen mittlere jährliche Einträge verschiedener Elemente berechnet werden. Die hierzu verwendeten Daten wurden zum größten Teil vom Niedersächsischen Landesamt für Ökologie (NLÖ) zur Verfügung gestellt. Dank für die gute Zusammenarbeit sei an dieser Stelle an Frau Gertsmeier und an Herrn Dr. Giesen gerichtet. Weitere Meßergebnisse lagen von verschiedenen Stationen des Forschungszentrums für Waldökosysteme (FZW) vor. Eine Zusammenstellung der verwendeten Stationen ist Anhang 3 zu entnehmen.

MEESEnBURG et al. (1994) stützen sich in ihrer Arbeit über die „Entwicklung der atmogenen Stoffeinträge in niedersächsische Waldbestände“ auf Meßwerte von Waldstationen, die auch mit anderem zeitlichem Rahmen für die vorliegende Untersuchung verwendet wurden. Sie beschreiben vor allem für Sulfat-Schwefel und Protonen einen Rückgang der Einträge von 1983 bis 1992. Auf eine erneute Darstellung des zeitlichen Trends der zur Verfügung stehenden Daten wird hier verzichtet. Vielmehr soll die räumliche Auswertung der Daten, die Regionalisierung für das Zielgebiet Niedersachsen beschrieben werden. Sie kann beispielsweise zur Verbesserung der Datengrundlage für die Anwendung eines wissensbasierten Systems zur Kalkungsplanung beitragen, wie es von JANSEN et al. (1994) beschrieben wurde.

In diesem Kapitel soll zunächst kurz auf die Prozesse eingegangen werden, die zur Deposition der verschiedenen Elemente führen (Kap. 4.2.1). Das Wissen um diese nutzte UlRICH (1991) zur Schätzung der Gesamtdepositionen in Wäldern. Daraufhin werden die verwendeten Datenkollektive vorgestellt. Ein deskriptiver Überblick und die Überprüfung der Repräsentanz der verwendeten Datenkollektive folgen, bevor 
Besonderheiten der Regionalisierung der Depositionen im Rahmen dieser Arbeit vorgestellt werden (Kap 4.2.4).

\subsubsection{Prozesse der Deposition}

Die jeweilige Erscheinungsform der Luftinhaltsstoffe - z. B. gas- oder partikelförmig - ist in starkem Maße von der Art des Emittenten abhängig. Sie hat Auswirkungen auf die Verweilzeit der Stoffe in der Luft und die zum Eintrag in Ökosysteme führenden Depositionsmechanismen. Hier sind zunächst zwei Prozesse zu unterscheiden: Nasse und trockene Deposition.

Die Stärke der trockenen Deposition wird maßgeblich durch physikalische Eigenschaften des Akzeptors (z. B. einer Blattoberfläche) und der Substanzen selbst beeinflußt (s. auch VDI 1987). CHAMBERLAIN (1975) prägte den Ausdruck der Depositionsgeschwindigkeit vd als Maß für die Effizienz der trockenen Deposition:

$$
\mathrm{vd}=\frac{\mathrm{F}}{\mathrm{C}(\mathrm{z})}
$$

vd - Depositionsgeschwindigkeit $(\mathrm{cm} / \mathrm{s})$

$\mathrm{C}(\mathrm{z})$ - Spurenstoffkonz. in Höhe z über der Erdoberfläche

F - Menge des auf einer Flächeneinheit deponierten Spurenstoffes

Die nasse Deposition umfaßt jede Ablagerung atmogener Stoffe durch die Niederschläge (s. auch VDI 1987). Die Spurenstoffkonzentration in der Luft, Niederschlagsintensität sowie die Tropfengrößenverteilung beeinflussen die Stoffkonzentrationen im Niederschlag maßgeblich. So werden z. B. in Nebel und Tau höhere Konzentrationen nachgewiesen als in großtropfigen Niederschlägen (WINKLER, 1983). IBROM (1993) konnte zeigen, daß die Konzentration von Protonen in Niederschlägen mit der Länge der vorhergehenden Trockenphasen steigt und mit fortlaufender Niederschlagsdauer abnimmt.

Die Depositionen werden an den Meßstellen flächendeckender Meßnetze im allgemeinen durch sogenannte Totalisatoren oder „Bulk-Sammler“ aufgefangen (vgl. z. B. MEesEnburg et al., 1994, oder NLÖ, 1993). MATZNER (1988, S. 19) teilt nach ULRICH (1983) die Gesamtdeposition in Niederschlagsdeposition (ND) und Interzeptionsdeposition (ID). Die Niederschlagsdeposition folgt den Gesetzen der Gravitation und ist somit leicht durch ständig offene Bulk-Sammler zu erfassen, 
wohingegen die Ermittlung der Interzeptionsdeposition als außerordentlich schwierige Aufgabe anzusehen ist. Letzere umfaßt „die Summe der über Adsorption von Gasen, Aerosolen (Trockene Deposition) und Wolken- bzw. Nebeltröpfchen ... im Kronenraum deponierten Elementmenge“. Die „Rate der Interzeptionsdeposition wird durch die Qualität der Akzeptoroberfläche stark beeinflußt“ (MATZNER, 1988). Sie macht in Waldbeständen häufig einen größeren Teil aus als die Niederschlagsdeposition.

$$
\mathrm{GD}_{\mathrm{X}}=\mathrm{ND}_{\mathrm{X}}+\mathrm{ID}_{\mathrm{X}}
$$

In den Begriff der Niederschlagsdeposition werden also neben den in Regen oder Schnee gelösten Stoffen auch die aus der Atmosphäre durch Sedimentation deponierten Stoffe eingeschlossen. Im Freiland entspricht die Niederschlagsdeposition somit der Gesamtdeposition und kann durch ständig offene Sammelbehälter direkt ermittelt werden.

\section{Abbildung 21: Schema der Depositionsmessungen durch Totalisatoren}

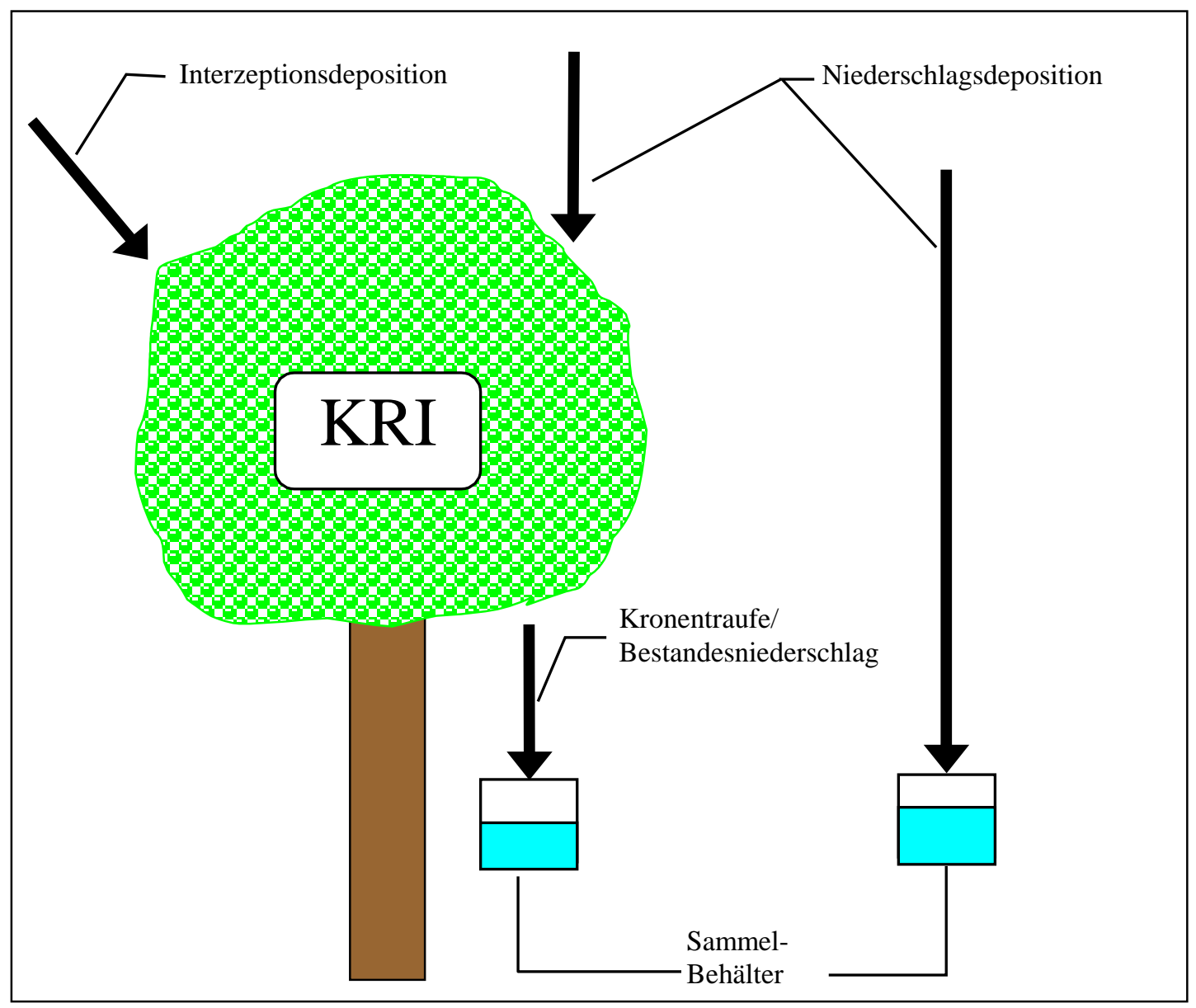


In Waldbeständen kann die Gesamtdeposition nicht als Bulk-Deposition ermittelt werden. Hier kommt es durch die große, durch Transpirationsvorgänge feuchte Akzeptoroberfläche der Kronen zu verstärkter Ausfilterung der durchstreifenden Luft und somit zu stärkerer ID als im Freiland. Wenn alle durch Interzeptionsdeposition eingetragenen Stoffe mit dem Niederschlag komplett abgewaschen würden, könnte man durch das Auffangen der Kronentraufe bzw. des Bestandesniederschlages die Gesamtdeposition auf sehr einfachem Weg ermitteln (vgl. ( 24 )). Eine Differenz zwischen GD und Kronentraufe/Bestandesniederschlag (BN) kann aber durch Kronenraum-Regen-Interaktionen (KRI) entstehen (s. Abbildung 21).

KRI umfassen sowohl die Aufnahme von Stoffen durch den Kronenraum (für $\mathrm{NH}_{4}$ und $\mathrm{NO}_{3}$ beispielsweise von IBROM, 1993, beobachtet) als auch die Auswaschung von Stoffen. Diese Auswaschung aus dem Kronenraum, das sogenannte „Leaching“, wurde auch für Nährstoffe beobachtet (z. B. IBROM, 1993). Da die Symptome der „,neuartigen Waldschäden“ häufig auf Magnesiummangel zurückgeführt werden (vgl. z. B. UBA, 1993, Bericht III, Kap. 2.3.1 oder ZöTTL et al., 1990), ist diesem Umstand erhöhte Aufmerksamkeit bei der Beurteilung der Ernährungslage der Wälder zu schenken.

Die Aufnahme von Stoffen im Kronenraum kann zu einer Unterschätzung der GD, das Leaching zur Überschätzung der GD durch den Bestandesniederschlag führen.

Nach der von ULRICH (1991) (in: FRIEDRICH, 1991) beschriebenen Berechnungsmethode ist es unter vereinfachenden Annahmen möglich, aus Daten des Bestandesniederschlages (Kronentraufe) und korrespondierenden Freilanddepositionen (Niederschlagsdeposition) die Gesamtdeposition im Bestand zu schätzen. Die Ergebnisse entsprechender Berechnungen wurden im Rahmen dieser Arbeit nicht zur Regionalisierung verwendet (vgl. Kap. 4.2.4 „Besonderheit der Regionalisierung der Depositionen“).

\subsubsection{Verwendete Datenkollektive}

Die Daten für die Depositionsregionalisierung stammen vom Niedersächsischen Landesamt für Ökologie (NLÖ) und vom Forschungszentrum für Waldökosysteme (FZW) der Universität Göttingen. Vom FZW standen Daten aus z.T. langjährigen Meßreihen einzelner, z.T. sehr intensiv untersuchter Gebiete zur Verfügung. 
Während die Verortung der NLÖ-Stationen durch Gauß-Krüger-Koordinaten gegeben war, mußten die Koordinaten für die meisten zur Verfügung stehenden FZW-Stationen durch ein Literaturstudium sowie Befragungen der beteiligten Forscher und Forstämter erarbeitet werden. Die genaue Ermittlung der geographischen Lage der Meßstationen sollte in aktuellen und zukünftigen Meßprogrammen einen höheren Stellenwert erfahren als bisher, da sie Grundlage für raumbezogene Analysen ist.

Die Messungen des NLÖ waren Teil eines Meßnetzes, das zum Ziel u. a. die „flächendeckende Ermittlung des Eintrages von Schadstoffen aus der Luft in Böden und Gewässer“ hat (NLÖ, 1992). „Um gebietsrepräsentative Ergebnisse zu erhalten“, wurden vom NLÖ mehrere Auswahlkriterien für Meßstandorte festgelegt (NLÖ, 1993). Eine flächendeckende Ermittlung des Eintrages wird folglich vom NLÖ angestrebt. Unterschieden werden Freilandmeßstellen und Bestandesmeßstellen. Grundsätzlich werden Bulk-Sammler eingesetzt. An den Freilandmeßstellen werden vom NLÖ sogenannte „Osnabrück-Sammler“ eingesetzt (Eigenkonstruktion des ehemaligen Niedersächsischen Landesamtes f. Wasser- und Abfallwirtschaft, NLWA, s. NLÖ, 1993). An Bestandesmeßstellen wird vom NLÖ der Niederschlagssammler des Typs „Münden 100“ verwendet (Selbstbau der Hess. Forstl. Versuchsanstalt Hann.-Münden, s. NLÖ, 1993). Nähere Angaben zur Probenahme, Lagerung und Analyse der Proben können dem Bericht des NLÖ zum Depositionsmeßnetz entnommen werden (NLÖ, 1993).

Die Beprobung, Probenlagerung und Analyse der vom FZW unterhaltenen Stationen ist weniger einheitlich geregelt als beim NLÖ (kein einheitliches Meßnetz sondern Einzelbeobachtungen) und variiert in Abhängigkeit vom Hauptuntersuchungsobjekt der bearbeitenden Wissenschaftler. Eine Liste der stationsspezifischen Literatur ist in Anhang 4 aufgeführt. Grundsätzlich erfolgte eine Aufstellung von 10 bis 20 systematisch verteilten Bulk-Sammlern, um die innerhalb des Bestandes variierenden Messungen für den ganzen Bestand mitteln zu können (vgl. XU \& BLANCK, 1994). In den Wintermonaten wurden Schneeimer eingesetzt. Die Probenahme erfolgte grundsätzlich alle zwei Wochen.

Nach KÖNIG (mdl. Mitteilung 1997) erbrachte eine Ringanalyse zwischen den in Deutschland mit entsprechenden Analysen befaßten Laboratorien Abweichungen von nur 5 bis $10 \%$. Die zu erwartenden Abweichungen durch ungeeignete Probenahme 
und -lagerung werden höher eingeschätzt. Unterschiedliche Analyseorte der gewonnenen Proben von NLÖ und FZW lassen daher keine systematischen Unterschiede zwischen den beiden Datenquellen erwarten. Es gibt weder durch den Vergleich der verwendeten Meßwerte noch durch deskriptive Statistiken zu den Meßwertverteilungen einen Anlaß zur Annahme von systematischen Unterschieden zwischen den hier verwendeten Datenquellen. Einzelne extreme Meßergebnisse können durch die Meßsituation der betreffenden Station erklärt werden und sind somit kein Grund für eine Eliminierung aus dem Datenkollektiv. Probenahme, Lagerung und Analyse der Daten sind ähnlich, so daß sie zu einer gemeinsamen statistischen Analyse herangezogen wurden.

\subsubsection{Zielvariablen der Depositionsregionalisierung}

Im Rahmen der vorliegenden Arbeit wurden die mehrjährigen Mittelwerte der Einträge von Sulfat-Schwefel $\left(\mathrm{SO}_{4}-\mathrm{S}\right)$, Nitrat- und Ammonium-Stickstoff $\left(\mathrm{NO}_{3}-\right.$ und $\left.\mathrm{NH}_{4}-\mathrm{N}\right)$, Chlor $\left(\mathrm{Cl}^{-}\right)$, Protonen $\left(\mathrm{H}^{+}\right)$sowie des $\mathrm{pH}-$ Wertes der gewonnenen Proben (Bulk-Depositionen) für die Jahre 1986 bis 1990 als Zielvariablen zur Regionalisierung verwendet. Für die im Rahmen des Lufthygiene Überwachungsnetzes (LÜN) unterhaltenen Meßstationen des NLÖ konnten keine Protoneneinträge, $\mathrm{pH}-$ Werte und Ammoniumeinträge für diese Untersuchungen zur Verfügung gestellt werden (vgl. Tabelle 5, S. 45). Zudem waren die $\mathrm{NH}_{4}-\mathrm{N}$ Meßreihe „Hude-Hasbruch“ und die $\mathrm{NO}_{3}-\mathrm{N}$ Meßreihe „Riefensbeck“ (beide NLÖ) nicht vollständig, weshalb sie bei den Untersuchungen nicht berücksichtigt wurden.

Daraus ergibt sich der in Tabelle 33 zusammengestellte Umfang der Datenkollektive.

Tabelle 33: Umfang der Datenkollektive (NLÖ und FZW gemeinsam) zur Regionalisierung mehrjähriger Mittelwerte für die Jahre 1986 bis 1990 von Stoffeintragsmessungen (Bulk-Depositionen)

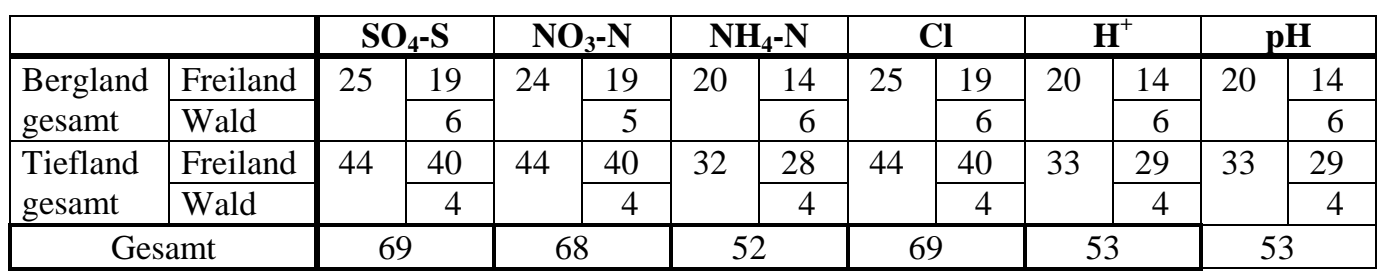

Die Repräsentanz der Depositionsdatenkollektive für gesamt Niedersachsen wird durch einen Vergleich der Werte räumlicher Variablen mit den entsprechenden Rasterdaten des Zielgebietes untersucht (Kap. 4.2.3). Einen Überblick über die Lage 
der Stationen gibt Abbildung 22. Deskriptive Statistiken zu den Zielvariablen sind in Tabelle 34 zusammengestellt.

Wie aus Abbildung 22 hervorgeht, sind die Stationen der drei Datenkollektive über ganz Niedersachsen verteilt. Schwerpunkte im Harz, im Solling, in der Heide und eine Meßnetzverdichtung im Südosten des Niedersächsischen Tieflandes (Braunschweig) sind zu erkennen.

Abbildung 22: Lage der zur Regionalisierung von Depositionen verwendeten Meßstationen

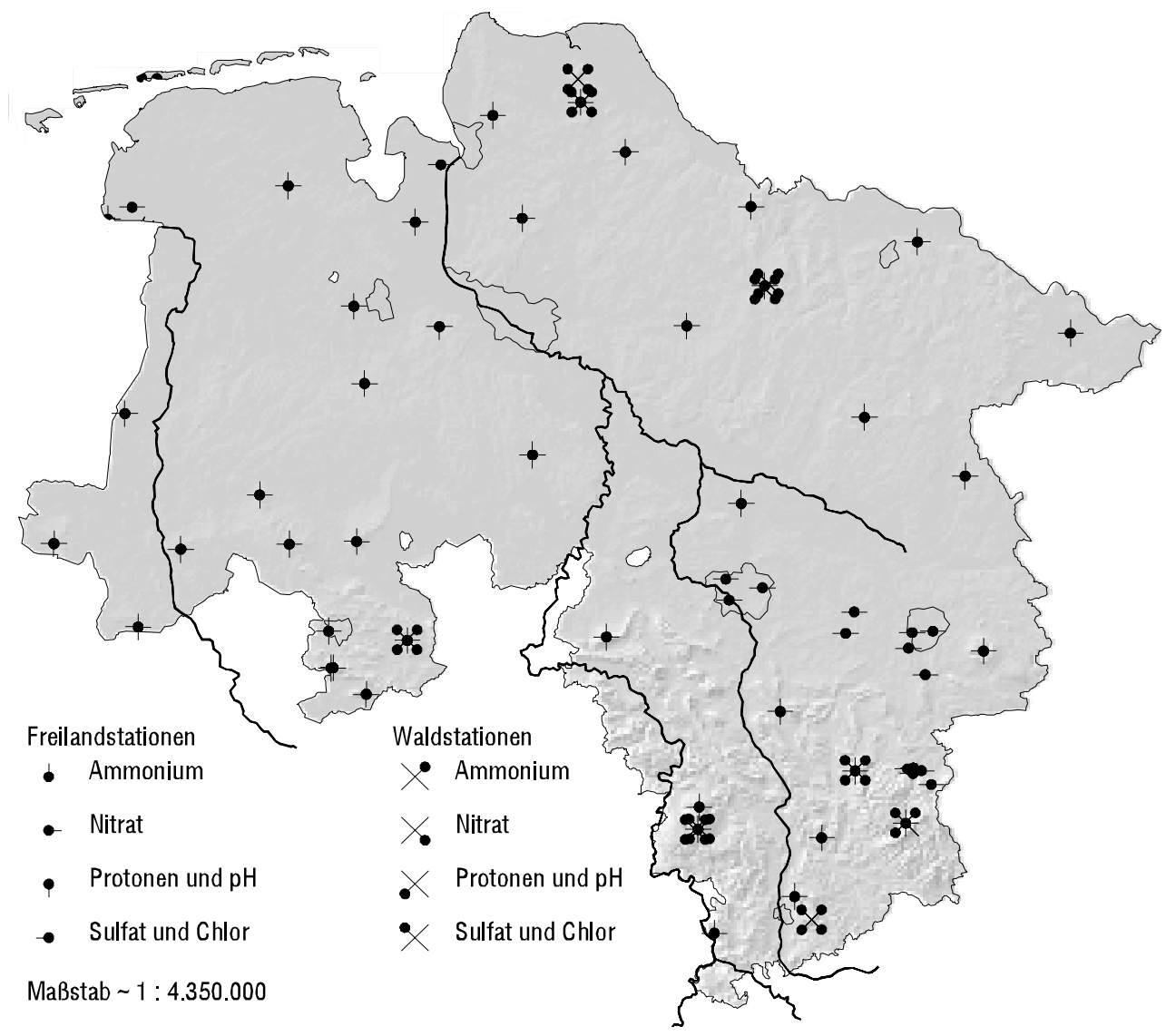


Tabelle 34: Deskriptive Statistiken zu den Depositionsmeßwerten

\begin{tabular}{|c|c|c|c|c|c|c|}
\hline Stationswerte & $\mathbf{n}$ & $\begin{array}{c}\text { Min. } \\
{\left[\mathrm{kg} /(\mathrm{ha} \bullet \mathrm{a})^{-1}\right]} \\
\text { bzw. pH }\end{array}$ & $\begin{array}{c}\text { Max. } \\
{\left[\mathrm{kg} /(\text { ha॰a })^{-1}\right]} \\
\text { bzw. pH }\end{array}$ & $\begin{array}{c}\text { Mittelw. } \\
{\left[\mathrm{kg} /(\text { ha॰a })^{-1}\right]} \\
\text { bzw. pH }\end{array}$ & \multicolumn{2}{|c|}{$\begin{array}{c}\text { Stdabw. } \\
\left.\text { [kg/(ha•a) }^{-1}\right] \\
\text { bzw.pH }\end{array}$} \\
\hline $\mathrm{SO}_{4}-\mathrm{S}$ gesamt & 69 & 8,7 & 154,3 & 20,4 & 20,9 & \\
\hline Freiland & 59 & 8,7 & 22,2 & 14,3 & & 3,3 \\
\hline Wald & 10 & 22,4 & 154,3 & 56,3 & & 39,5 \\
\hline $\mathrm{NO}_{3}-\mathrm{N}$ gesamt & 68 & 4,4 & 38,2 & 7,2 & 4,8 & \\
\hline Freiland & 59 & 4,4 & 9,4 & 6,0 & & 1,1 \\
\hline Wald & 9 & 6,2 & 38,2 & 15,6 & & 9,8 \\
\hline $\mathrm{NH}_{4}-\mathrm{N}$ gesamt & 52 & 4,8 & 42,6 & 12,2 & 9,0 & \\
\hline Freiland & 42 & 4,8 & 13,9 & 8,8 & & 2,1 \\
\hline Wald & 10 & 10,7 & 42,6 & 26,1 & & 12,9 \\
\hline gesamt & 69 & 6,7 & 133,5 & 28,1 & 30,8 & \\
\hline Freiland & 59 & 6,7 & 133,5 & 22,3 & & 25,2 \\
\hline Wald & 10 & 18,7 & 129,9 & 62,2 & & 39,2 \\
\hline gesamt & 53 & 0,02 & 3,1 & 0,3 & 0,6 & \\
\hline Freiland & 43 & 0,02 & 0,6 & 0,2 & & 0,1 \\
\hline Wald & 10 & 0,12 & 3,1 & 1,0 & & 1,1 \\
\hline gesamt & 53 & 3,6 & 5,5 & 4,6 & 0,4 & \\
\hline Freiland & 43 & 4,2 & 5,5 & 4,7 & & 0,3 \\
\hline Wald & 10 & 3,6 & 4,6 & 4,1 & & 0,4 \\
\hline
\end{tabular}

Wie aus Tabelle 34 hervorgeht, sind die Unterschiede zwischen den Freiland- und den Walddepositionen z.T. enorm. Nur für Chlor wurden an einer Freilandstation (,Norderney Stadt“) höhere Einträge gemessen als an Waldmeßstationen (Max.: „Riefensbeck“). Bis auf die Verteilung des Chlor-Freilandeintrages zeigen alle Einträge und der pH-Wert in den Freilanddepositionen ein annähernd normalverteiltes Bild. Die Verteilung der Chloreinträge ist geprägt durch die hohen Meßwerte von drei Stationen (,Emden-Knock“, „Norderney Flughafen“ und „Norderney Stadt“). Diese Stationen weisen Werte für den Chloreintrag auf $\left(111,5 \mathrm{~kg}(\mathrm{ha} \bullet)^{-1}\right.$ bis $\left.133,5 \mathrm{~kg}(\mathrm{ha} \bullet \mathrm{a})^{-1}\right)$, die erheblich über denen anderer Freilandstationen liegen $\left(44,7 \mathrm{~kg}\right.$ (ha•a) ${ }^{-1}$,Wingst Abt. 28 Freifläche“).

Die Waldstation „Riefensbeck“ weist neben enormen Chloreinträgen $\left(129,9 \mathrm{~kg}(\mathrm{ha} \bullet)^{-1}\right)$ auch den maximalen Eintrag von Sulfat-Schwefel auf. Dieser liegt fast doppelt so hoch wie der Wert der Station mit den zweithöchsten Sulfateinträgen (,Solling F1-Fläche“). Die Höhe der Einträge ist durch die Lage der Station „Riefensbeck“ am Nordwesthang des „Ackers“ im Harz in einer Geländehöhe von ca. $730 \mathrm{~m}$ ü. NN und der damit verbundenen hohen Exponiertheit für West- und Nordwestwinde erklärbar.

Im Falle des Ammonium-Stickstoffs wurden für die Waldmeßstation „Wingst Abt. 28“ mit 42,6 kg (ha•a) ${ }^{-1}$ höhere Einträge gemessen als für „Riefensbeck“ $\left(38,7 \mathrm{~kg}(\mathrm{ha} \bullet \mathrm{a})^{-1}\right)$. Dies wiederum kann durch die isolierte Lage des Waldgebietes 
Wingst in einer Region intensiver Landwirtschaft und Tiermast erklärt werden (vgl. MEESENBURG et al., 1994).

Den maximalen mittleren Eintrag an Nitrat-Stickstoff weist die Station „Seesen“ des NLÖ auf $\left(38,2 \mathrm{~kg}(\mathrm{ha} \bullet)^{-1}\right)$. Die nächstniedrigeren Einträge sind mit

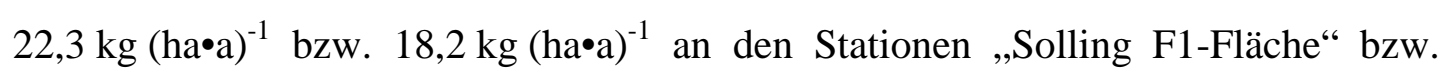
„Buer-Ostenwalde M.55“ gemessen worden. Der Mittelwert der Station „Riefensbeck“ für den mittleren Eintrag von Nitrat-Stickstoff von 1986 bis 1990 konnte wegen fehlender Daten für 1989 zwar nicht ermittelt werden, die vorhandenen Jahresfrachten liegen jedoch alle zwischen 61 und $99 \mathrm{~kg}(\mathrm{ha} \bullet)^{-1}$. Dies kommt im Vergleich zur Station „Seesen“ einem zwei- bis dreimal so hohen Eintrag gleich.

Die im Mittel sauersten Bulk-Depositionen wurden für die Waldstationen „Solling F1-Fläche“, „Seesen“ und „Riefensbeck“ ermittelt (3,61, 3,63 und 3,77). Neun Freilandstationen weisen im Mittel pH-Werte über 5 auf, wobei die Depositionen an der Station „Norderney Stadt“ mit einem mittleren $\mathrm{pH}$-Wert von 5,53 am schwächsten sauer waren.

Die stärksten Protoneneinträge wurden für die Freilandstationen an der Solling F1Fläche ermittelt $\left(0.58 \mathrm{~kg}(\mathrm{ha} \bullet)^{-1}\right)$, die geringsten für die Stationen „Norderney Stadt“. Auch für die Waldstationen gibt es erwartungsgemäß ähnliche Ergebnisse wie für den pH-Wert. Die am stärksten belasteten Stationen sind „Seesen“, „Solling F1-Fläche“ und „Riefensbeck“ in dieser Reihenfolge mit 1,8 kg(ha•a) ${ }^{-1}$, $2,6 \mathrm{~kg}\left(\mathrm{ha} \bullet \mathrm{a}^{-1}\right.$ und $3,1 \mathrm{~kg}(\mathrm{ha} \bullet)^{-1}$. Die Waldstation mit den geringsten mittleren Protoneneinträgen im Bestandesniederschlag ist die Station „Wingst Abt. 28“ mit nur $0,1 \mathrm{~kg}(\mathrm{ha} \cdot \mathrm{a})^{-1}$.

Nur von 11 Stationen (7 Waldstationen, 4 Freilandstationen des FZW) konnten für den Untersuchungszeitraum (1986 bis 1990) mittlere Natrium-Einträge berechnet werden. Die Depositionen der NLÖ-Stationen werden erst seit 1988 auf ihren Natrimgehalt hin analysiert. Wegen des geringen Stichprobenumfangs und einer sehr ungleichmäßigen räumlichen Verteilung der Stationen - die entsprechenden Stationen sind auf Forschungsschwerpunkte des FZW konzentriert - wurde auf eine Regionalisierung der Natriumeinträge im Rahmen dieser Arbeit verzichtet.

Schon die Betrachtung der Extrem- und Mittelwerte macht den Unterschied zwischen Freiland- und Walddepositionen deutlich. Eindeutiger wird dies noch durch 
die Betrachtung der Streuung. Alle beobachteten Depositionen und der pH-Wert zeigen für die Waldstationen eine deutlich höhere Standardabweichung. Eine gemeinsame Regionalisierung mit der in Kap. 2.5 beschriebenen Methodik scheidet aus, weil die Koeffizienten angepaßter Regressionsmodelle im wesentlichen durch die enorm hohe Varianz der Waldstandorte geprägt würden. Es wurde daher eine für Wald- und Freilandstationen getrennte bzw. gestaffelte Regionalisierung angestrebt, wie sie in Kap. 4.2.4 beschrieben wird.

\subsubsection{Repräsentanz der Depostionsdatenkollektive}

Wie bereits geschildert (s. Tabelle 33, S. 90), wurde nicht an allen Stationen das gleiche Meßprogramm durchgeführt. Die meisten Beobachtungen lagen von 69 Stationen für den mittleren Eintrag von Sulfat-Schwelfel und Chlor vor. Bis auf eine Station (Waldstation „Riefensbeck“") lagen für diese Stationen auch die mittleren Nitrat-Stickstoffeinträge vor. Unter Angabe der Lageparameter von „Riefensbeck“ und deskriptiver Statistiken des Nitratdatenkollektivs wurden diese drei Datenkollektive gemeinsam bezüglich ihrer Repräsentanz für das Untersuchungsgebiet überprüft.

Der Vergleich zwischen den Verteilungen der Rasterwerte für ganz Niedersachsen einerseits und den Verteilungen der für die Meßstationen ermittelten Werte räumlicher Variablen andererseits (s. Tabelle 35) zeigt eine weitestgehende Repräsentanz der drei Datenkollektive (Nitrat-Stickstoff, Chlor und Sulfat-Schwefel) für das Untersuchungsgebiet Niedersachsen. Es muß jedoch darauf hingewiesen werden, daß beispielsweise für die Hangneigung und den Index für Leewirbel in Niedersachsen deutlich höhere Werte vorkommen als durch die Datenkollektive vertreten werden (Vergleich der Extrema). Auf diese Problematik wurde bereits in Kap. 4.1.2.1 eingegangen (s. Seite 66), weshalb eine nähere Erläuterung an dieser Stelle unterbleibt. 
Tabelle 35: Gegenüberstellung von Verteilungen räumlicher Variablen (deskriptive Statistiken) für Niedersachsen und Nitratstationen; in Klammern der Anteil nicht repräsentierter Flächen über/unter dem Maximum/Minimum

\begin{tabular}{|c|c|c|c|c|c|c|c|c|}
\hline \multicolumn{4}{|c|}{$\begin{array}{c}\text { Niedersachsen } \\
\text { (Rasterwerte; Rasterweite: } \mathbf{5 0} \text { m) }\end{array}$} & \multirow[t]{2}{*}{ Variable } & \multicolumn{4}{|c|}{$\begin{array}{c}\text { Nitratstationen }(\mathrm{n}=68) \\
\text { (Repräsentanzmängel in \%) }\end{array}$} \\
\hline Min. & Max. & Mittelw. & Stdabw. & & Min. & Max. & Mittelw. & Stdabw. \\
\hline-11 & 953 & 63,57 & 92,49 & $\begin{array}{l}\text { Geländehöhe } \\
\text { [m ü. NN] }\end{array}$ & $\begin{array}{r}0 \\
(0,0)\end{array}$ & $\begin{array}{r}754 \\
(0,1)\end{array}$ & 149,61 & 166,27 \\
\hline 0 & 136 & 2,17 & 4,99 & $\begin{array}{l}\text { Hangneigung } \\
{[\%]}\end{array}$ & $\begin{array}{r}0 \\
(0,0)\end{array}$ & $\begin{array}{r}26 \\
(1,0)\end{array}$ & 3,99 & 5,41 \\
\hline 0 & 369 & 109,43 & 14,68 & $\begin{array}{c}\text { Insolation Januar } \\
\text { [ ] }\end{array}$ & $\begin{array}{r}63 \\
(1,5) \\
\end{array}$ & $\begin{array}{r}203 \\
(0,2)\end{array}$ & 111,51 & 20,52 \\
\hline 0 & 36 & 35,53 & 2,50 & $\begin{array}{l}\text { Relative Exponiertheit } \\
\text { (0 bis } 36)\end{array}$ & $\begin{array}{r}13 \\
(0,2)\end{array}$ & $\begin{array}{r}36 \\
(0,0)\end{array}$ & 34,68 & 4,10 \\
\hline 0 & 2000 & 131,85 & 375,35 & $\begin{array}{l}\text { Kaltluftabfluß } \\
(0 \text { bis } 2000)\end{array}$ & $\begin{array}{r}0 \\
(0,0) \\
\end{array}$ & $\begin{array}{l}2000 \\
(0,0)\end{array}$ & 145,10 & 434,19 \\
\hline 0 & 1096 & 27,78 & 61,138 & $\begin{array}{l}\text { Leewirbel } \\
{[\mathrm{m}]}\end{array}$ & $\begin{array}{r}0 \\
(0,0)\end{array}$ & $\begin{array}{r}609 \\
(0,1)\end{array}$ & 46,04 & 100,81 \\
\hline
\end{tabular}

Wegen der auffälligen Unterschiede zwischen Freilanddepositionen und Bestandesniederschlägen (s. Kap. 4.2.2.1), ist eine getrennte Betrachtung der Repräsentanz nötig. Die Freilandkollektive für die Einträge von Sulfat-Schwefel, Nitrat-Stickstoff und Chlor bestehen aus Daten von 59 Stationen. Die Variablenverteilung dieser Freilandstationen und die der 9 Waldstationen - im Falle des Sulfat-Schwefels und des Chloreintrages durch Station „Riefensbeck“ ergänzt (vgl. Tabelle 39) - werden in Tabelle 36 gegenübergestellt.

Tabelle 36: Gegenüberstellung von Variablenverteilungen (deskriptive Statistiken) für Niedersachsen und Nitratstationen; getrennt für 59 Freilandstationen und 9 Waldstationen; (für Station „Riefensbeck“ zusätzlich Sulfat-Schwefel- und Chloreintrag bekannt)

\begin{tabular}{|c|c|c|c|c|c|c|c|c|c|}
\hline \multicolumn{4}{|c|}{$\begin{array}{l}\text { Niedersachsen (Rasterwerte; } \\
\text { Rasterweite: } 50 \text { m) }\end{array}$} & \multirow[t]{2}{*}{ Variable } & \multicolumn{3}{|c|}{$\begin{array}{l}\text { Freilandstationen } \\
\text { Waldstationen } \mathrm{NO}_{3}-\mathrm{N}\end{array}$} & $\begin{array}{l}(n=59) \\
(n=9)\end{array}$ & \multirow{2}{*}{\begin{tabular}{|c|}
$\begin{array}{l}\text { Riefens- } \\
\text { beck }\end{array}$ \\
Werte \\
\end{tabular}} \\
\hline Min. & Max. & Mittelw. & Stdabw. & & Min. & Max. & Mittelw. & Stdabw. & \\
\hline \multirow[t]{2}{*}{-11} & 953 & 63,57 & 92,49 & Geländehöhe & 0 & 754 & 132,22 & 152,70 & \\
\hline & & & & [m ü. NN] & $25^{\circ}$ & $507^{-}$ & 263,61 & 213,63 & 732 \\
\hline \multirow[t]{2}{*}{0} & 136 & 2,17 & 4,99 & Hangneigung & 0 & 26 & 3,93 & 5,57 & \\
\hline & & & & {$[\%]$} & 0 & $15^{-}$ & 4,37 & 4,43 & 13 \\
\hline \multirow[t]{2}{*}{0} & 369 & 109,43 & 14,68 & Insolation Jan. & 63. & 203 & 111,15 & 21,08 & \\
\hline & & & & {[]} & $94^{\circ}$ & 150 & 113,90 & 17,18 & 77 \\
\hline \multirow[t]{2}{*}{0} & 36 & 35,53 & 2,50 & Rel. Exponiertheit & 13 & 36 & 34,69 & 4,13 & \\
\hline & & & & (0 bis 36$)$ & $24^{-}$ & $36^{-}$ & 34,63 & 4,12 & 31 \\
\hline \multirow[t]{2}{*}{0} & 200 & 131,85 & 375,35 & Kaltluftabfluß & 0. & 2000 & 166,02 & 463,02 & \\
\hline & 0 & & & $(0$ bis 2000$)$ & 0 & 45 & 8,00 & 14,06 & 5 \\
\hline \multirow[t]{2}{*}{0} & 109 & 27,78 & 61,138 & Leewirbel & 0 & 609 & 48,64 & 107,79 & \\
\hline & 6 & & & {$[\mathrm{~m}]$} & 0 & 56 & 28,99 & 22,14 & 0 \\
\hline
\end{tabular}

Die relativen Repräsentanzmängel im Vergleich zu den Variablenverteilungen in ganz Niedersachsen wurden in Tabelle 35 für die Gesamtkollektive in Klammern 
dargestellt. Sie gelten wegen der identischen Extremwerte auch für die Verteilungen der Freilandkollektive. Die Betrachtung der Verteilungen der Lageparameter der 9 Nitrat-Waldstationen zeigt einen deutlich kleineren Wertebereich. Auch die Verteilungen für die 10 Waldstationen des Sulfat-Schwefel- und des Chlorkollektivs weisen durch die Integration der Station „Riefensbeck“ nur bezüglich der Geländehöhe und der Insolation im Januar ein breiteres Wertespektrum auf (s auch Tabelle 40, S. 98). Die relativen Repräsentanzmängel der 9 Nitrat-Waldstationen werden der Übersichtlichkeit wegen separat in Tabelle 37 dargestellt.

Tabelle 37: Anteil der nicht durch den Wertebereich der 9 NitratWaldstationen repräsentierten Rasterpunkte in Niedersachsen

\begin{tabular}{|c|c|c|}
\hline Variable & $\begin{array}{c}\text { unter Min. } \\
{[\%]}\end{array}$ & $\begin{array}{c}\text { über Max. } \\
{[\%]}\end{array}$ \\
\hline $\begin{array}{c}\text { Geländehöhe } \\
\text { [m ü. NN] }\end{array}$ & 33,7 & 0,9 \\
\hline $\begin{array}{c}\text { Hangneigung } \\
\text { [\%] }\end{array}$ & 0,0 & 3,0 \\
\hline $\begin{array}{c}\text { Insolation Jan. } \\
\text { [ ] }\end{array}$ & 5,6 & 1,6 \\
\hline $\begin{array}{c}\text { Rel. Exponiertheit } \\
\text { (0 bis 36) }\end{array}$ & 1,44 & 0,0 \\
\hline $\begin{array}{c}\text { Kaltluftabfluß } \\
\text { (0 bis 2000) }\end{array}$ & 0,0 & 26,0 \\
\hline $\begin{array}{c}\text { Leewirbel } \\
\text { [m] }\end{array}$ & 0,0 & 14,3 \\
\hline
\end{tabular}

Gut ein drittel der niedersächsischen Fläche wird demnach die Geländehöhe betreffend nicht durch diese 9 Stationen repräsentiert. Auch die anderen hier aufgeführten Variablen betreffend zeigen sich bedeutende Repräsentanzmängel. Bei der Regionalisierung der Bestandesniederschläge ist so neben der geringen Zahl von Waldmeßstationen mit nicht repräsentativer Baumartenverteilung auch noch der Mangel an Repräsentanz räumlicher Situationen zu berücksichtigen.

Gegenüber den 53 Stationen mit mittleren pH-Werten und Protoneneinträgen fehlt im Ammoniumdatenkollektiv lediglich die Freilandstation „Hude-Hasbruch“. Die Lage der Stationen kann Abbildung 22 (S. 91) entnommen werden. Es ist auffällig, daß von vielen Stationen der zuvor untersuchten Datenkollektive, die in drei Gebieten - im Harz, im Bergland allgemein und in der Gegend um Braunschweig liegen, für die Analyse der hier dargestellten Zielvariablen keine ausreichenden Meßreihen zur Verfügung standen.

Es folgt eine Darstellung der Ergebnisse einer Repräsentanzuntersuchung bezüglich des Ammoniumdatenkollektivs (Freiland- und Waldstationen). Die Lageparameter 
der Freilandstation „Hude-Hasbruch“, für die zusätzlich auch Mittelwerte für Protoneneinträge und $\mathrm{pH}-\mathrm{Werte}$ der Einträge vorliegen, sind in Tabelle 39 gesondert aufgeführt.

Tabelle 38: Gegenüberstellung von Verteilungen (deskriptive Statistiken) der Rasterwerte für Niedersachsen und der Variablenwerte der Ammoniumstationen

\begin{tabular}{|c|c|c|c|c|c|c|c|c|}
\hline \multicolumn{4}{|c|}{$\begin{array}{c}\text { Niedersachsen } \\
\text { (Rasterwerte; Rasterweite: } \mathbf{5 0} \text { m) }\end{array}$} & \multirow[t]{2}{*}{ Variable } & \multicolumn{4}{|c|}{$\begin{array}{c}\text { Ammoniumstationen }(\mathrm{n}=52) \\
\text { (Repräsentanzmängel in \%) }\end{array}$} \\
\hline Min. & Max. & Mittelw. & Stdabw. & & Min. & Max. & Mittelw. & Stdabw. \\
\hline-11 & 953 & 63,57 & 92,49 & $\begin{array}{l}\text { Geländehöhe } \\
\text { [m ü. NN] }\end{array}$ & $\begin{array}{r}0 \\
(0,0)\end{array}$ & $\begin{array}{r}754 \\
(0,1)\end{array}$ & 175,04 & 198,27 \\
\hline 0 & 136 & 2,17 & 4,99 & $\begin{array}{c}\text { Hangneigung } \\
{[\%]}\end{array}$ & $\begin{array}{r}0 \\
(0,0) \\
\end{array}$ & $\begin{array}{r}26 \\
(1,0) \\
\end{array}$ & 4,68 & 5,93 \\
\hline 0 & 369 & 109,43 & 14,68 & $\begin{array}{c}\text { Insolation Januar } \\
{[]}\end{array}$ & $\begin{array}{r}63 \\
(1,5)\end{array}$ & $\begin{array}{r}203 \\
(0,2)\end{array}$ & 112,54 & 22,58 \\
\hline 0 & 36 & 35,53 & 2,50 & $\begin{array}{l}\text { Rel. Exponiertheit } \\
\text { (0 bis 36) }\end{array}$ & $\begin{array}{r}17 \\
(0,2)\end{array}$ & $\begin{array}{r}36 \\
(0,0)\end{array}$ & 34,85 & 3,43 \\
\hline 0 & 2000 & 131,85 & 375,35 & $\begin{array}{c}\text { Kaltluftabfluß } \\
(0 \text { bis } 2000)\end{array}$ & $\begin{array}{r}0 \\
(0,0) \\
\end{array}$ & $\begin{array}{l}2000 \\
(0,0)\end{array}$ & 98,65 & 365,13 \\
\hline 0 & 1096 & 27,78 & 61,138 & $\begin{array}{l}\text { Leewirbel } \\
{[\mathrm{m}]}\end{array}$ & $\begin{array}{r}0 \\
(0,0)\end{array}$ & $\begin{array}{r}172 \\
(4,3)\end{array}$ & 23,50 & 34,08 \\
\hline
\end{tabular}

Für das Ammonium-Gesamtdatenkollektiv kann weitestgehend Repräsentanz festgestellt werden. Lediglich die Variable Leewirbel weist auf bedeutende Repräsentanzmängel hin. Dies kann durch die geringere Zahl an Berglandstationen in entsprechender Lage im Vergleich zu den drei zuerst untersuchten Datenkollektiven erklärt werden.

Die Lageparameter der Station „Hude-Hasbruch“ (s. Tabelle 39) weisen auf keinerlei Besonderheiten hin. Die Aufnahme dieser Station in das $\mathrm{H}^{+}-$und das $\mathrm{pH}-$ Datenkollektiv hat daher die Repräsentanz betreffend keine bedeutende Veränderung gegenüber derjenigen des Ammonium-Gesamtdatenkollektivs zur Folge.

Die getrennte Betrachtung von Wald- und Freilandstationen zeigt weitere Repräsentanzmängel in den Teilkollektiven. Da Mittelwerte aller 10 Waldstationen (vgl. Sulfat-Schwefel und Chlor oben) für die zwei hier betrachteten Stoffe (Ammonium-Stickstoff und Protonen) sowie den pH-Wert zur Verfügung standen, kann eine einheitliche Darstellung der Waldstationen erfolgen. Für die Freilandstationen werden die Verteilungen der Ammoniumstationen $(n=42)$ dargestellt, welche im Falle des Protoneneintrages und des pH-Wertes durch die Werte der Station „Hude-Hasbruch“ (s. Tabelle 39) ergänzt werden müssen. 
Tabelle 39: Gegenüberstellung von Variablenverteilungen (deskriptive Statistiken) für Niedersachsen und Ammoniumstationen; getrennt für 42 Freilandstationen und 10 Waldstationen; für Station ,HudeHasbruch" lagen zudem Protoneneinträge und pH-Werte vor

\begin{tabular}{|c|c|c|c|c|c|c|c|c|c|}
\hline \multicolumn{4}{|c|}{$\begin{array}{l}\text { Niedersachsen (Rasterwerte } \\
\text { Rasterweite: } 50 \text { m) }\end{array}$} & \multirow[t]{2}{*}{ Variable } & \multicolumn{4}{|c|}{$\begin{array}{ll}\text { Freilandstationen } & (\mathrm{n}=42) \\
\text { Waldstationen } \mathrm{NO}_{3}-\mathrm{N} & (\mathrm{n}=10)\end{array}$} & \multirow{2}{*}{$\begin{array}{c}\begin{array}{c}\text { H.-Has- } \\
\text { bruch }\end{array} \\
\text { Werte } \\
\end{array}$} \\
\hline Min. & Max. & Mittelw. & Stdabw. & & Min. & Max. & Mittelw. & Stdabw. & \\
\hline \multirow[t]{2}{*}{-11} & \multirow[t]{2}{*}{953} & \multirow[t]{2}{*}{63,57} & \multirow[t]{2}{*}{92,49} & \multirow{2}{*}{$\begin{array}{l}\text { Geländehöhe } \\
\text { [m ü. NN] }\end{array}$} & 0 & 754 & 142,82 & 172,21 & 24 \\
\hline & & & & & 25 & 732 & 310,40 & 249,92 & \\
\hline \multirow[t]{2}{*}{0} & \multirow[t]{2}{*}{136} & \multirow[t]{2}{*}{2,17} & \multirow[t]{2}{*}{4,99} & \multirow{2}{*}{$\begin{array}{c}\text { Hangneigung } \\
{[\%]}\end{array}$} & 0 & 26 & 4,48 & 6,07 & 1 \\
\hline & & & & & 0 & 16 & 5,53 & 5,55 & \\
\hline \multirow[t]{2}{*}{0} & \multirow[t]{2}{*}{369} & \multirow[t]{2}{*}{109,43} & \multirow[t]{2}{*}{14,68} & \multirow{2}{*}{$\begin{array}{c}\text { Insolation Jan. } \\
\text { [ ] }\end{array}$} & 63 & 203 & 113,10 & 23,34 & 107 \\
\hline & & & & & 77 & 150 & 110,18 & 20,02 & \\
\hline \multirow[t]{2}{*}{0} & \multirow[t]{2}{*}{36} & \multirow[t]{2}{*}{35,53} & \multirow[t]{2}{*}{2,50} & \multirow{2}{*}{$\begin{array}{l}\text { Rel. Exponiertheit } \\
\text { (0 bis } 36)\end{array}$} & 17 & 36 & 34,99 & 3,30 & 36 \\
\hline & & & & & 24 & 36 & 34,25 & 4,06 & \\
\hline \multirow[t]{2}{*}{0} & \multirow[t]{2}{*}{2000} & \multirow[t]{2}{*}{131,85} & \multirow[t]{2}{*}{375,35} & \multirow{2}{*}{$\begin{array}{l}\text { Kaltluftabfluß } \\
(0 \text { bis } 2000)\end{array}$} & 0 & 2000 & 120,32 & 404,10 & 7 \\
\hline & & & & & 0 & 45 & 7,66 & 13,30 & \\
\hline \multirow[t]{2}{*}{0} & \multirow[t]{2}{*}{1096} & \multirow[t]{2}{*}{27,78} & \multirow[t]{2}{*}{61,138} & \multirow{2}{*}{$\begin{array}{c}\text { Leewirbel } \\
{[\mathrm{m}]}\end{array}$} & 0 & 172 & 22,88 & 36,45 & 6 \\
\hline & & & & & 0 & 56 & 26,09 & 22,80 & \\
\hline
\end{tabular}

Die Extrema der für die Freilandstationen ermittelten Variablenwerte entsprechen denen des Gesamtdatenkollektivs, Mittelwerte und Standardabweichungen sind zumindest sehr ähnlich. Es wird daher bei der nach Freiland- und Waldstationen getrennten Darstellung auf eine gesonderte Diskussion der Repräsentanz der Freilandstationen verzichtet. Die relativen Repräsentanzmängel der Waldstationen sind z.T. erheblich größer, weshalb sie separat in Tabelle 40 dargestellt werden.

Tabelle 40: Anteil der nicht durch den Wertebereich der 10 Waldstationen (alle Zielvariablen außer Ammonium-Stickstoff) repräsentierten Rasterpunkte in Niedersachsen

\begin{tabular}{|c|c|c|}
\hline Variable & $\begin{array}{c}\text { unter Min. } \\
{[\%]}\end{array}$ & $\begin{array}{c}\text { über Max. } \\
{[\%]}\end{array}$ \\
\hline $\begin{array}{c}\text { Geländehöhe } \\
\text { [m ü. NN] }\end{array}$ & 33,7 & 0,1 \\
\hline $\begin{array}{c}\text { Hangneigung } \\
{[\%]}\end{array}$ & 0,0 & 2,7 \\
\hline $\begin{array}{c}\text { Insolation Jan. } \\
{[\text { ] }}\end{array}$ & 2,5 & 1,6 \\
\hline $\begin{array}{c}\text { Rel. Exponiertheit } \\
\text { (0 bis 36) }\end{array}$ & 1,4 & 0,0 \\
\hline $\begin{array}{c}\text { Kaltluftabfluß } \\
\text { (0 bis 2000) }\end{array}$ & 0,0 & 26,0 \\
\hline $\begin{array}{c}\text { Leewirbel } \\
{[\mathrm{m}]}\end{array}$ & 0,0 & 14,3 \\
\hline
\end{tabular}

Es zeigt sich - ähnlich wie im Fall der 9 Nitrat-Stickstoff-Waldstationen -, daß zumindest für einige Variablen erhebliche Einschränkungen der Repräsentanz bestehen. 
Für die Freilanddatenkollektive kann nahezu volle Repräsentanz festgestellt werden (s. Tabelle 35 und Tabelle 38), während für die 9 bzw. 10 Waldstationen (s. Tabelle 37 und Tabelle 40) erhebliche Einschränkungen bestehen. Dieser Umstand wird zusammen mit dem geringen Stichprobenumfang und der nicht repräsentativen Baumartenverteilung der Walddatenkollektive zu diskutieren sein (Kap. 6.2.2).

Eine für Berg- und Tiefland getrennte Repräsentanzuntersuchung macht nur für die Freilandstationen Sinn. Sie wird hier nicht ausführlich dargestellt, da im Vordergrund der Untersuchungen im Rahmen der vorliegenden Arbeit Aussagen über den Waldbestand im Untersuchungsgebiet stehen sollten und diese bestenfalls für gesamt Niedersachsen abgeleitet werden können. Eine tabellarische Gegenüberstellung, wie sie auch in den vorherigen Kapiteln verwendet wurde, findet sich in Anhang 5 bis Anhang 8.

Es zeigt sich im Bergland eine Unterrepräsentanz der Freilandstationen für steile Lagen (Hangneigung $>26 \%$ ) sowie für Lagen mit geringer Insolation im Januar (<63). Für das Ammonium-Berglandkollektiv (ebenfalls nur Freilandstationen) zeigt sich zudem eine bedeutende Unterrepräsentanz für hohe Leewirbel- und Kaltluftabflu $\beta$-Werte sowie eine Repräsentanzlücke für ebene Lagen (Hangneigung=0\%).

Obwohl die Extrema der Tieflandkollektive teilweise stark von denen der Rasterwerte abweichen, kann flächenmäßig weitestgehende Repräsentanz der Freilandmeßstellen für das Untergebiet festgestellt werden. Lediglich Lagen mit sehr geringer Insolation im Januar (<102) sind mit einer Repräsentanzlücke von 4,5\% auffällig.

\subsubsection{Besonderheit der Regionalisierung der Depositionen}

Neben der enormen Unterschiedlichkeit von Freiland- und Walddepositionen sind die Unterschiede zwischen Berg- und Tiefland bei den Überlegungen zur Modellbildung zu berücksichtigen. Wie in Kap. 5.1.2 dargestellt wird, sind in Bergund Tiefland verschiedene Modelle zur Regionalisierung der durchschnittlichen Niederschlagssummen entwickelt worden. Da auch die nasse Deposition und ein Teil der Prozesse der trockenen Deposition mit dem Niederschlag zusammenhängen 
(z. B. Feuchtigkeit von Blattoberflächen durch Benetzung/Transpiration, Wasserregime der Pflanzen; s. z. B. BurkHARD, 1994 in: AG-DePosition, 1994 oder GRAF \& SCHMID, 1989), sollte grundsätzlich auch die Regionalisierung von Depositionen in Freiland- und Bestandesniederschlägen nach Möglichkeit getrennt für Berg- und Tiefland erfolgen.

Wie bei den meisten Analysen ist neben der Frage nach dem Wunsch auch die Frage der Machbarkeit auf Grundlage der Datenbasis zu klären. Eine sinnvolle räumliche Analyse sollte sich auf ausreichend umfangreiche Datenkollektive stützen. Wie Tabelle $33 \mathrm{zu}$ entnehmen ist, sind bezüglich der Freilandstationen für Berg- und Tiefland stets ähnlich umfangreiche Datenkollektive verfügbar wie im Falle der Lufttemperatur- und Feuchteanalysen (vgl. Kap. 4.1.2 und Kap. 4.1.4). Eine auf wenige Regressoren beschränkte Modellbildung konnte für die Freilanddepositionen getrennt nach Berg- und Tiefland durchgeführt werden (s. S. 138ff).

Da Aussagen über die vom Freiland abweichende Depositionssituation in Waldbeständen gemacht werden sollten, war dies mit den nur in geringer Zahl zur Verfügung stehenden Daten für eine Trennung nach Berg- und Tiefland - nach der in Kap. 2.5 beschriebenen Methodik - nicht möglich. Mit insgesamt sechs Stationen unter Fichte, zweien unter Buche und jeweils einer unter Kiefer und unter Eiche ist die Baumartenverteilung grundsätzlich nicht geeignet, Unterschiede für einzelne Baumarten quantifizieren zu können. Zudem entspricht die Baumartenverteilung nicht derjenigen des Untersuchungsraumes. Laubbaumbestände und vor allem die Eiche sind unterrepräsentiert.

Um eine Verringerung des Umfangs der wenigen (Wald-) Daten für den Untersuchungszeitraum zu vermeiden, wurden die Regionalisierungsmodelle für die räumliche Verteilung der untersuchten Depositionen für gesamt Niedersachsen ermittelt. Nur so konnte eine annähernd ausreichende Zahl an Waldstationen von neun (Nitrat) bzw. zehn (andere Elemente) für die statistischen Analysen verwendet werden.

Zunächst wurden für jedes Element an die Daten aller Freilandstationen lineare Regressionsmodelle angepaßt, wie in Kap. 2.5 beschrieben (Ergebnisse in Kap. 5.2.1). Die Verwendung des (regionalisierten) Jahresniederschlages als potentieller Regressor sollte eine getrennte Untersuchung nach Berg- und Tiefland ersetzen, da die Niederschlagsmodelle bereits Unterschiede zwischen Berg- und 
Tiefland berücksichtigen (Kap. 5.1.2). Die Niederschlagssumme findet hier Verwendung als Index für das gesamte Wasserregime. Alle nicht mit der Niederschlagssumme in Zusammenhang stehenden Prozesse werden als einheitlich für Berg- und Tiefland angenommen.

Den größten Unterschied zwischen Wald- und Freilanddepositionen stellen Kronenraum-Regen-Interaktionen und die im Wald bedeutenden Interzeptionsdepositionen - nicht durch die Schwerkraft bestimmte Depositionen (vgl. MATZNER, 1988, S.19) - dar. Es ist plausibel, daß diese Interzeptionsprozesse maßgeblich zu den bereits deskriptiv beschriebenen Unterschieden von Wald- und Freilanddepositionen beigetragen haben (Tabelle 34). LENZ et al. (1996) beschreiben die Abweichung der Wald- von den Freilanddepositionen über sogenannte Anreicherungsfaktoren. Die Höhe der Anreicherungsfaktoren (bei LENZ et al., 1996: Verhältnis Gesamtdepositionen im Wald zu Freilanddepositionen am selben Ort) wurde durch schrittweise lineare Regression mit Standortsfaktoren als Regressoren regionalisiert (Bestand, Topographie, Wind- und Nebelhäufigkeit). Der Mangel an treffsicheren und über mehrere Bundesländer hinweg konsistenten Modellen wurde von LENZ et al. (1996) dadurch erklärt, „daß diejenigen Faktoren, die lokal das Depositionsgeschehen bestimmen, nur unzureichend oder überhaupt nicht .... erfaßt werden konnten.“ Dies ist ein Mangel, der durch die auf hohe Lagegenauigkeit der Stationen bedachte Recherche und die GIS-gestützte Generierung von räumlichen Variablen im Rahmen der vorliegenden Arbeit minimiert werden sollte. Zur Abschätzung der Gesamtdepositionen wurde von LENZ et al. (1996) folgender Algorithmus empfohlen:

Gesamtdeposition $_{\text {Bestand }}=$ Deposition $_{\text {Freiland }} *\left(\right.$ Fakt $_{\text {Bestand }}+$ Fakt $_{\text {Nebel }}+$ Fakt $\left._{\text {Expo }}\right)$

Die vorliegende Arbeit verwendet einen vereinfachten Ansatz. Da Bestandesniederschläge von nur 10 Waldstationen zur Verfügung standen, sollte nur EIN Anreicherungsfaktor regionalisiert werden. Baumart oder zumindest Baumartengruppen konnten über das Landnutzungsraster (Kap. 3.4.1) für das Zielgebiet Niedersachsen und über Literaturangaben und Recherchen bei den für die Meßstationen zuständigen Forstämtern für die Waldmeßstationen bestimmt werden. Unter der Annahme, daß die Nebelhäufigkeit als klimatisches Element bei geeigneter Datenbasis mit der in Kap. 2.5 beschriebenen Methodik regionalisiert und damit 
durch räumliche Variablen beschrieben werden kann, steht auch einer direkten Regionalisierung EINES (Gesamt-) Anreicherungsfaktors nichts entgegen.

Hier wurde der Anreicherungsfaktor von Freilanddepositionen zu Bestandesniederschlägen (Fakt ${ }_{\text {ges }}$ ) als Zielvariable gewählt, um die Regionalisierungsvariable frei von Unsicherheiten durch die Berechnung der Gesamtdepositionen zu halten:

$$
\text { Bestandesniederschlag }=\text { Deposition }_{\text {Freiland }} * \text { Fakt }_{\text {ges }}
$$

Dieser Anreicherungsfaktor konnte für die in Kap. 4.2.2.1 genannten fünf Stoffe und den pH-Wert als Quotient aus den gemessenen Bestandesniederschlägen und den für Niedersachsen regionalisierten Freilanddepositionen berechnet werden. Die regionalisierten Freilanddepositionen mußten hier verwendet werden, da nicht für alle Waldstationen Werte von korrespondierenden Freilanddepositionen vorlagen.

Die Regionalisierung der Depositionsdaten erfolgte somit für die Einträge von Sulfat-Schwefel, Ammonium-Stickstoff, Nitrat-Stickstoff, Chlor, Protonen und für den $\mathrm{pH}-$ Wert in zwei Schritten. Im ersten wurden die mittleren Freilanddepositionen (1986 bis 1990) für das Zielgebiet Niedersachsen durch schrittweise lineare Regression regionalisiert. Im zweiten Schritt erfolgte die Regionalisierung der Bestandanreicherungsfaktoren, die sich allerdings nur auf Werte von 10 bzw. 9 (Nitrat-Stickstoff) Waldstationen stützen konnte. 


\section{Ergebnisse}

Die Gliederung des Kapitels entspricht der im Kapitel 4, „Verwendete Daten“. Alle Zielvariablen wurden zunächst mit Hilfe räumlicher Variablen regionalisiert. Im Falle der durchschnittlichen Niederschlagssummen konnten die Residuen der auf diesem Weg gefundenen Regressionsmodelle als Basis einer zusätzlichen geostatistischen Analyse verwendet werden (Kap. 2.5).

Die Ergebnisdarstellung umfaßt für die Regressionsmodelle neben der Darstellung der Regressionskoeffizienten eine Beschreibung der Anpassungsgüte an die zugrundeliegenden Daten in Form von Bestimmtheitsmaß $\left(\mathrm{R}^{2}\right)$ und rooted mean squared error (RMSE).

Im Fall der monatlichen Niederschlagssummen werden die Ergebnisse der weiterführenden geostatistischen Analyse in Form der Regressionskoeffizienten der angepaßten GLS-Modelle (generalized least squares) und der Parameter der Semivariogramme angegeben, welche die räumliche Autokorrelation beschreiben. Zur Quantifizierung der Präzision werden die Ergebnisse von Kreuzvalidierungen dargestellt.

\subsection{Klimaelemente}

Für die Klimaelemente wurde eine getrennte Regionalisierung nach Berg- und Tiefland durchgeführt. In diesem Kapitel sollen die allgemeinen Gründe für die Trennung des gesamtniedersächsischen Datenkollektivs aufgezeigt werden, bevor mit der eigentlichen Ergebnisdarstellung in den Unterkapiteln begonnen wird.

Zwei Gründe sprechen für eine Teilung des Zielgebietes und der zugehörigen Stationen in ein Bergland- und ein Tieflanddatenkollektiv: Plausibilität und Präzision. In Abbildung 16 auf Seite 70 wurde die Reliefierung Niedersachsens dargestellt. Ein relativ ebenes Tiefland, geprägt durch die Nähe zum Meer und 
fehlende orographische Barrieren für aus Westen oder Norden anströmende Luftmassen, ist deutlich verschieden vom niedersächsischen Bergland mit seinen bis $1000 \mathrm{~m}$ hohen Gebirgen (Harz). Auch in der Literatur werden die starken Unterschiede zwischen diesen beiden Landesteilen beschrieben (s. ARBEITSKREIS STANDORTKARTIERUNG, 1985).

Eine sinnvolle Grenze zwischen Bergland und Tiefland konnte von ScHulz (in Vorb.) durch eine Clusteranalyse anhand der Informationslagen Geländehöhe, Hangneigung und Relative Exponiertheit ermittelt werden (s. Abbildung 16). Diese wurde zur graphischen Umsetzung der Modelle verwendet. Zur zeitlich früher erfolgten Einteilung der Meßstationen in Bergland- und Tieflanddatenkollektive war aus Mangel an objektiven Kriterien eine gerade Grenzführung gewählt worden. Den Berglandkollektiven wurden alle Stationen südlich des Hochwertes 5803000 und östlich des Rechtswertes 3495000 zugeschlagen, die restlichen Stationen bildeten die Tieflandkollektive (s. Abbildung 23).

Auch wenn einige Stationen z. B. des Berglanddatenkollektivs der Lufttemperatur ebenso gut im Tieflandkollektiv hätten verwendet werden können, so wird eine gewisse Überschreitung der ,wahren“ Grenze, für die es immer nur Annäherungen wie diejenige nach SCHULZ (in Vorb.) geben kann, nicht als Problem angesehen. Sollen die für die Teilgebiete ermittelten Regionalisierungsmodelle zu einem Gesamtmodell integriert werden, kann eine „grenzüberschreitende“ Zuordnung von Stationen dazu beitragen, die Modelle nicht zu unähnlich werden zu lassen. Auch die Verwendung grenznaher Stationen in beiden Datenkollektiven wäre denkbar gewesen. Die Frage, wie die Modelle an den Grenzen der Untergebiete modifiziert werden sollen, um einen gleitenden Übergang zwischen den Modellen zu erreichen, wurde durch eine distanzabhängige Mittelung der Modellergebnisse in einem $10 \mathrm{~km}$ breiten Übergangsbereich gelöst. 


\section{Abbildung 23: Einteilung in Bergland- und Tieflanddatenkollektive; Grenze gutachterlich festgelegt.}

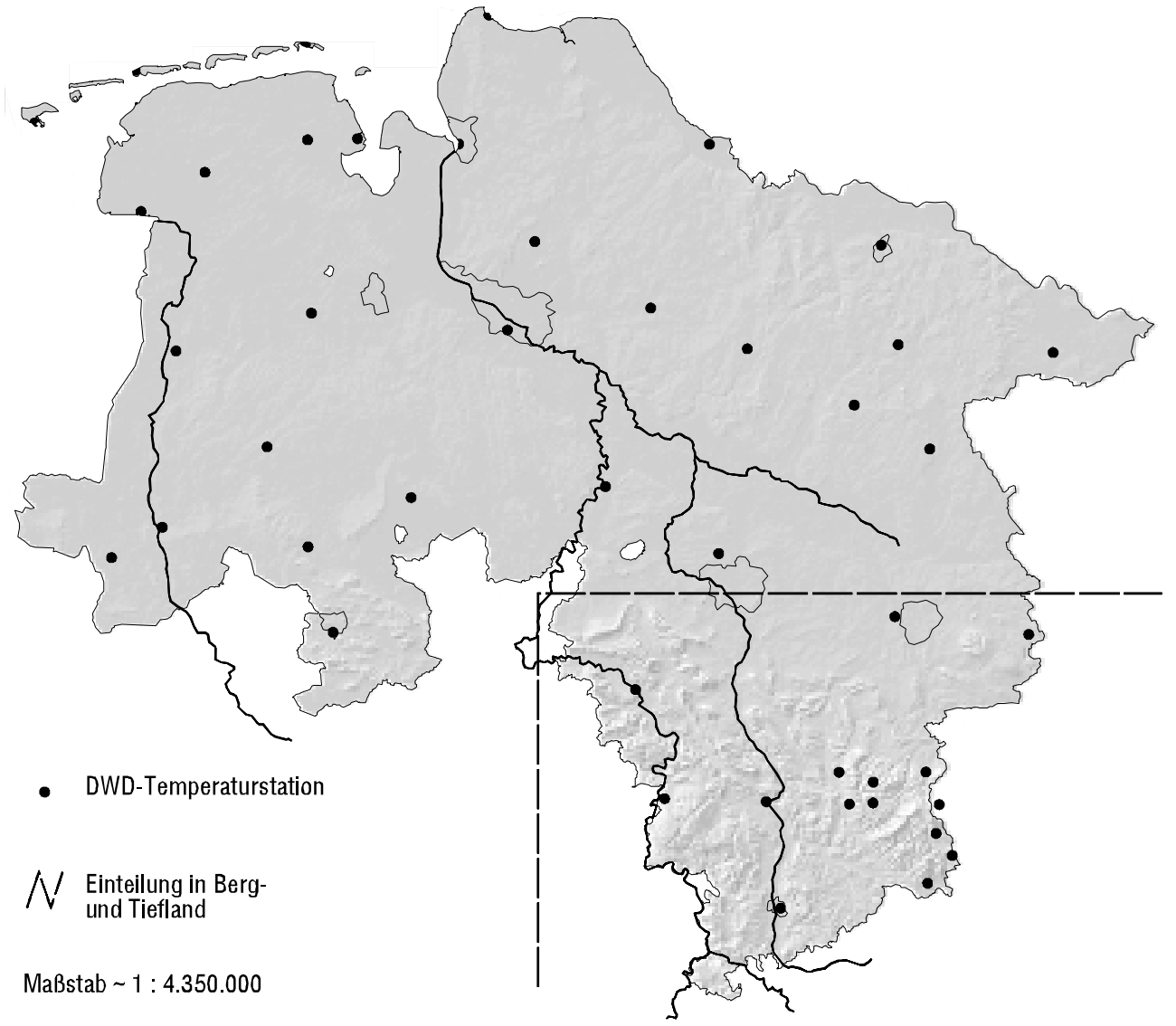

Die Notwendigkeit der Einteilung in Teilkollektive ist unter anderem mit dem Vergleich einiger geomorphologischer Größen von Bergland und Tiefland (Rasterwerte) $\mathrm{zu}$ begründen. In Tabelle 41 zeigen sich für alle dargestellten Variablen deutliche Unterschiede der Verteilungen. So sind zwar beispielsweise Extremwerte und Mittelwerte der Verteilungen der Strahlungsvariable Insolation Januar relativ ähnlich, ihre Standardabweichungen unterscheiden sich jedoch stark, was die höhere Reliefenergie des Berglandes zum Ausdruck bringt. 
Tabelle 41: Gegenüberstellung von Variablenverteilungen (deskriptive Statistiken) für Tiefland und Bergland

\begin{tabular}{|c|c|c|c|c|c|c|c|c|}
\hline \multicolumn{4}{|c|}{$\begin{array}{c}\text { Bergland } \\
\text { (Rasterwerte; Rasterweite: } 50 \mathrm{~m} \text { ) }\end{array}$} & \multirow[t]{2}{*}{ Variable } & \multicolumn{4}{|c|}{$\begin{array}{c}\text { Tiefland } \\
\text { (Rasterwerte; Rasterweite: } \mathbf{5 0} \mathbf{~ m} \text { ) }\end{array}$} \\
\hline Min. & Max. & Mittelw. & Stdabw. & & Min. & Max. & Mittelw. & Stdabw. \\
\hline 35 & 953 & 218,23 & 130,75 & $\begin{array}{l}\text { Geländehöhe } \\
\text { [m ü. NN] }\end{array}$ & -11 & 172 & 35,53 & 28,35 \\
\hline 0 & 136 & 8,34 & 8,97 & $\begin{array}{c}\text { Hangneigung } \\
{[\%]}\end{array}$ & 0 & 63 & 0,68 & 1,34 \\
\hline 0 & 368 & 107,61 & 33,91 & $\begin{array}{c}\text { Insolation Januar } \\
{[]}\end{array}$ & 0 & 306 & 108,95 & 5,06 \\
\hline 0 & 36 & 33,25 & 5,50 & $\begin{array}{l}\text { Rel. Exponiertheit } \\
(0-36)\end{array}$ & 0 & 36 & 35,99 & 0,28 \\
\hline 0 & 2000 & 92,25 & 311,76 & $\begin{array}{l}\text { Kaltluftabfluß } \\
\quad(0-2000)\end{array}$ & 0 & 2000 & 138,01 & 398,09 \\
\hline 0 & 1096 & 110,10 & 110,67 & $\begin{array}{l}\text { Leewirbel } \\
{[\mathrm{m}]}\end{array}$ & 0 & 406 & 12,04 & 18,74 \\
\hline
\end{tabular}

Größer sind die Unterschiede im Falle derjenigen Variablen die in direkterem Zusammenhang mit der Geländehöhe stehen. Neben dieser Variable selbst zeigen so vor allem Hangneigung und Leewirbel stark unterschiedliche Verteilungen für die beiden Untergebiete.

Es erscheint angesichts der Reliefverhältnisse plausibel, anzunehmen, daß im Bergland durchaus andere Faktoren maßgeblich zur Variabilität von Klimaelementen beitragen als im Tiefland. Neben der Plausibilität kann eine verbesserte Präzision zur Begründung einer getrennten Regionalisierung herangezogen werden. Während die gebotene Einfachheit der Regionalisierung den Verzicht auf (überflüssige) Unterteilung des Zielgebietes fordert, ist eine höhere Präzision der Modelle in Untergebieten ein weiteres Argument für eine getrennte Regionalisierung. Dieser Aspekt soll zu Beginn der Kapitel der einzelnen regionalisierten Elemente dargestellt werden.

\subsubsection{Lufttemperatur}

Die Präzision der individuellen Berg- und Tieflandmodelle für die durchschnittliche Lufttemperatur bzw. der Modelle für das gesamte Niedersachsen wurde anhand der Summen der quadrierten Residuen (SQR) der Regressionsmodelle verglichen. Es gilt die Summen der Residuenquadrate der Berglandstationen (Tieflandstationen) bei Anwendung des Gesamtmodells mit denen der Berglandstationen (Tieflandstationen) bei Anwendung des spezifischen Berglandmodells (Tieflandmodell) zu vergleichen. 
Hinter den Werten der SQR wurde die Zahl der zur Modellbildung herangezogenen räumlichen Variablen - in Klammern - aufgeführt, um auch die Beurteilung der Einfachheit der Modelle zu ermöglichen (s. Tabelle 43).

Die Modelle für das Tiefland werden in Kapitel 5.1.1.2 noch ausführlich erläutert. In den der Tabelle 43 zugrunde liegenden DWD-Modellen für das Bergland wird die Lufttemperatur ausschließlich durch die Geländehöhe der Meßstationen erklärt (Tabelle 44, Kap. 5.1.1.1). Die Geländehöhe ist auch in allen Modellen für das gesamte Niedersachsen enthalten (Tabelle 42). Zusätzlich werden die Modelle für Dezember und Januar durch den Rechtswert, für März und April durch den Hochwert und für die Monate von Mai bis August durch den Abstand zur Küste gebildet. Den größten Anteil an der Varianzaufklärung hat in allen Modellen für das Gesamtdatenkollektiv die Geländehöhe.

Die Bestimmtheitsmaße der Gesamtmodelle sind mit Werten von $86 \%$ (Oktober) bis knapp $98 \%$ (April) als sehr hoch zu bezeichnen. Auch die Werte des RMSE zeigen mit $0,19{ }^{\circ} \mathrm{C}$ (April) bis $0,43{ }^{\circ} \mathrm{C}$ (September) eine sehr gute Anpassung der Modelle an die Daten. Die Standardabweichungen der Meßdaten (vgl. Tabelle 1, Seite 8) waren immerhin um einen Faktor von 2,7 (Oktober) bis 6,8 (April) größer als der durch den RMSE ausgedrückte Modellfehler.

Tabelle 42: Regressionskoeffizienten, Bestimmtheitsmaße $\left(\mathbf{R}^{2}\right)$ und Wurzel der mittleren quadrierten Residuen (RMSE) der Modelle für monatliche und jährliche mittlere Lufttemperatur in Niedersachsen

\begin{tabular}{|c|c|c|c|c|c|c|c|}
\hline $\begin{array}{c}\text { Monat/ } \\
\text { Jahr }\end{array}$ & $\begin{array}{c}\mathbf{R}^{2} \\
{[\%]}\end{array}$ & $\begin{array}{c}\text { RMSE } \\
{\left[1 / 10{ }^{\circ} \mathrm{C}\right]}\end{array}$ & $\begin{array}{c}\text { Additive } \\
\text { Konstante }\end{array}$ & Geländehöhe & Rechtswert & Hochwert & $\begin{array}{l}\text { Abstand } \\
\text { z. Küste }\end{array}$ \\
\hline Jan & 94,2 & 3,06 & 174,79 & $-0,0411$ & $-4,73 \mathrm{E}-05$ & & \\
\hline Feb & 90,5 & 3,93 & 15,16 & $-0,0527$ & & & \\
\hline Mär & 96,2 & 2,62 & 352,37 & $-0,0665$ & & $-5,28 \mathrm{E}-05$ & \\
\hline Apr & 97,9 & 1,91 & 601,09 & $-0,0712$ & & $-8,91 \mathrm{E}-05$ & \\
\hline Mai & 95,5 & 2,74 & 118,38 & $-0,0719$ & & & $9,01 \mathrm{E}-05$ \\
\hline Jun & 96,1 & 2,56 & 149,53 & $-0,0719$ & & & $8,97 \mathrm{E}-05$ \\
\hline Jul & 96,4 & 2,36 & 163,96 & $-0,0691$ & & & $8,18 \mathrm{E}-05$ \\
\hline Aug & 93,6 & 3,01 & 164,40 & $-0,0610$ & & & $5,35 \mathrm{E}-05$ \\
\hline Sep & 87,6 & 4,26 & 139,20 & $-0,0492$ & & & \\
\hline Okt & 86,3 & 4,17 & 99,83 & $-0,0454$ & & & \\
\hline Nov & 90,4 & 4,08 & 54,14 & $-0,0545$ & & & \\
\hline Dez & 94,3 & 3,17 & 176,75 & $-0,0445$ & $-4,40 \mathrm{E}-05$ & & \\
\hline Jahr & 92,3 & 3,35 & 90,37 & $-0,0503$ & & & \\
\hline
\end{tabular}


Tabelle 43: Vergleich von Summen der quadrierten Residuen (SQR) für gemeinsame Modellierung (MOD: NDS) und für getrennte Modelle der Lufttemperatur (MOD: TIEF, bzw. MOD: BERG); in Klammern: Anzahl der Regressoren im jeweiligen Modell

\begin{tabular}{|c|c|c|c|c|c|c|c|c|c|}
\hline \multirow[b]{2}{*}{$\begin{array}{c}\text { Stationen } \\
\mathbf{N} \\
\end{array}$} & \multicolumn{3}{|c|}{ MOD: NDS } & \multicolumn{2}{|c|}{ Vorteil spez. Modell } & \multicolumn{4}{|c|}{ MOD: TIEF MOD: BERG } \\
\hline & $\begin{array}{c}\text { Gesamt } \\
45\end{array}$ & \multirow{2}{*}{$\begin{array}{c}\text { Tiefland } \\
\mathbf{2 9} \\
133\end{array}$} & \multirow{2}{*}{$\begin{array}{c}\text { Bergland } \\
\mathbf{1 6} \\
260\end{array}$} & \multirow{2}{*}{$\begin{array}{c}\text { Tiefland } \\
24\end{array}$} & Bergland & \multicolumn{2}{|c|}{$\begin{array}{c}\text { Tiefland } \\
29\end{array}$} & \multicolumn{2}{|c|}{$\begin{array}{c}\text { Bergland } \\
16\end{array}$} \\
\hline Jan & $393 \quad(2)$ & & & & 42 & 109 & $(1)$ & 218 & (1) \\
\hline Feb & 664 & 510 & 154 & 428 & 6 & 82 & $(2)$ & 148 & (1) \\
\hline Mär & 289 & 180 & 109 & 100 & 6 & 80 & $(3)$ & 103 & (1) \\
\hline Apr & 154 & 88 & 66 & 21 & 1 & 67 & $(3)$ & 65 & (1) \\
\hline Mai & 315 & 248 & 67 & 114 & -30 & 134 & $(3)$ & 97 & (1) \\
\hline Jun & 276 & 210 & 66 & 115 & -39 & 95 & (3) & 105 & (1) \\
\hline Jul & 233 & 137 & 96 & 76 & -36 & 61 & (3) & 132 & (1) \\
\hline Aug & 379 & 275 & 104 & 171 & -6 & 104 & (3) & 110 & (1) \\
\hline Sep & 780 & 495 & 285 & 379 & 178 & 117 & (3) & 108 & (1) \\
\hline Okt & 746 & 607 & 139 & 470 & 34 & 137 & (3) & 106 & (1) \\
\hline Nov & 716 & 563 & 153 & 426 & 8 & 137 & (2) & 145 & (1) \\
\hline Dez & 422 & 170 & 252 & 55 & 11 & 115 & (2) & 240 & (1) \\
\hline Jahr & 481 & 262 & 219 & 201 & 153 & 62 & (3) & 66 & (1) \\
\hline
\end{tabular}

Obwohl das Gesamtmodell für den Januar zwei Variablen verwendet und die Teilkollektivmodelle jeweils nur eine, sind diese von höherer Präzision! Lediglich in den Monaten Mai bis August ist das Gesamtmodell besser an die Berglanddaten angepaßt als das allerdings einfachere Berglandmodell. Vor allem die Verbesserung der Beschreibung der Tieflanddaten durch spezielle Modelle rechtfertigt die Unterteilung des Zielgebietes. Auch bei gleicher Anzahl von einbezogenen Regressoren (s. Dezember) ist das Tieflandmodell besser an die Tieflanddaten angepaßt.

Somit sprechen sowohl Plausibilität als auch Präzision der Modelle zur Regionalisierung der Lufttemperatur für eine Teilung des Zielgebietes, eine für Bergund Tiefland getrennte Regionalisierung. Die Ergebnisse für beide Teilgebiete werden in den folgenden Kapiteln dargestellt.

\subsubsection{Lufttemperatur im Bergland}

Zur Regionalisierung der Lufttemperatur im niedersächsischen Bergland konnte neben dem DWD Berglanddatenkollektiv auch ein Datenkollektiv des Institutes für Bioklimatologie (TRANSEKT) herangezogen werden (Kap. 4.1.2.1). Während die Daten des DWD-Kollektivs lediglich einen sehr starken Zusammenhang zwischen der Lufttemperatur und der Geländehöhe mit sehr hohen Bestimmtheitsmaßen ergaben - vgl. Tabelle 44-, zeigte bei der Analyse der TRANSEKT-Daten 108 
zusätzlich die räumliche Variable QWKaltluftabfluß (Kap. 3.3.6) ein hohes Erklärungspotential, was durch die höhere Repräsentanz dieses Datenkollektivs für kaltluftexponierte Lagen erklärt werden kann (Kap. 4.1.2.1). Es schien vorteilhaft, auch die Erkenntnisse aus der Analyse dieses Meßnetzes mit anderem Beobachtungszeitraum zu berücksichtigen (Messungen aus den Jahren 1994 und 1995), da durch die ortsfernen Stationen dieses Kollektivs eine Erhöhung der räumlichen Repräsentanz der Modelle zu erhoffen ist (Kap. 4.1.2.1).

Tabelle 44: Regressionskoeffizienten, Bestimmtheitsmaße $\left(\mathbf{R}^{2}\right)$ und Wurzel der mittleren quadrierten Residuen (RMSE) der Modelle für monatliche und jährliche mittlere Lufttemperatur im niedersächsischen Bergland nach DWD-Datenkollektiv (16 Stationen)

\begin{tabular}{l|cc|rc}
\hline $\begin{array}{c}\text { Monat/ } \\
\text { Jahr }\end{array}$ & $\begin{array}{c}\mathbf{R}^{\mathbf{2}} \\
{[\%]}\end{array}$ & $\begin{array}{c}\text { RMSE } \\
{\left[\mathbf{1 / 1 0}{ }^{\circ} \mathbf{C}\right]}\end{array}$ & $\begin{array}{c}\text { Additive } \\
\text { Konstante }\end{array}$ & $\begin{array}{c}\text { Gelände- } \\
\text { höhe }\end{array}$ \\
\cline { 1 - 2 } Jan & 93,3 & 3,95 & 9,51 & $-0,0493$ \\
Feb & 96,1 & 3,26 & 16,06 & $-0,0539$ \\
Mär & 98,1 & 2,71 & 47,78 & $-0,0641$ \\
Apr & 98,9 & 2,16 & 87,99 & $-0,0689$ \\
Mai & 98,2 & 2,64 & 135,11 & $-0,0656$ \\
Jun & 98,1 & 2,74 & 166,05 & $-0,0655$ \\
Jul & 97,5 & 3,07 & 179,92 & $-0,0646$ \\
Aug & 97,6 & 2,81 & 175,81 & $-0,0596$ \\
Sep & 97,5 & 2,77 & 144,42 & $-0,0579$ \\
Okt & 96,6 & 2,75 & 102,07 & $-0,0488$ \\
Nov & 96,4 & 3,22 & 55,17 & $-0,0557$ \\
\cline { 2 - 2 } Dez & 93,3 & 4,14 & 22,63 & $-0,0515$ \\
\hline Jahr & 98,5 & 2,18 & 95,22 & $-0,0586$ \\
\hline
\end{tabular}

Zur integrierten Analyse beider Datenkollektive wurde eine Regressionsanalyse unter Einbeziehung der Dummy-Variable TRA durchgeführt. Sie wurde für alle TRANSEKT-Stationen mit dem Wert 1, für die DWD-Stationen mit dem Wert 0 belegt. Das mit den Daten der beiden Kollektive parametrisierte Modell ist:

$\mathrm{TEM}=\beta_{0}+\beta_{1} * \mathrm{TRA}+\beta_{2} *$ Geländehöhe $+\beta_{3} * \mathrm{TRA} *$ Geländehöhe $+\beta_{4} *$ QWKaltluftabfluß ( 27 )

Der Koeffizient der Dummy-Variable TRA, $\beta_{1}$, zeigt die mittlere Abweichung des TRANSEKT-Datenkollektivs vom DWD-Datenkollektiv an. Die additive Konstante des Regressionsmodells, $\beta_{0}$, ist somit unbeeinflußt von den Meßwerten des TRANSEKT-Datenkollektivs. Ebenso unbeeinflußt bleibt der Regressionskoeffizient der Variable Geländehöhe $\left(\beta_{2}\right)$, da die Interaktion zwischen der Dummy-Variable und dieser Variable, TRA*Geländehöhe, in das Modell integriert ist. Da die Dummy- 
Variable TRA für DWD-Stationen den Wert 0, für die TRANSEKT-Stationen aber den Wert 1 hat, gibt der Regressionskoeffizient der Interaktion die Abweichung der Höhenabhängigkeit im TRANSEKT-Datenkollektiv von der im DWDDatenkollektiv an. Der Einfluß der Variable QWKaltluftabflu $\beta\left(\beta_{4}\right)$ wird durch beide Datenkollektive bestimmt.

Durch die ausschließliche Verwendung der additiven Konstante und der Regressionskoeffizienten von Geländehöhe und $Q$ WKaltluftabflu $\beta$ zur späteren Regionalisierung der Lufttemperatur gehen die Messungen des TRANSEKTDatenkollektivs so lediglich bei der Ermittlung des Koeffizienten für $Q W K a l t l u f t a b f l u \beta$ ein. Dies erschien notwendig, um den möglichen Fehler durch den abweichenden und kürzeren Beobachtungszeitraum der TRANSEKT-Daten zu minimieren.

Tabelle 45: Regressionskoeffizienten und Koeffizienten der DummyKomponenten, Bestimmtheitsmaße $\left(\mathbf{R}^{2}\right)$ und Wurzel der mittleren quadrierten Residuen (RMSE) der Modelle für monatliche und jährliche mittlere Lufttemperatur im niedersächsischen Bergland nach kombiniertem Datenkollektiv (DWD und TRANSEKT) bzw. für Januar bis März ausschließlich nach DWD-Datenkollektiv

\begin{tabular}{|c|c|c|c|c|c|c|c|}
\hline $\begin{array}{c}\text { Monat/ } \\
\text { Jahr }\end{array}$ & $\begin{array}{c}\mathrm{R}^{2} \\
{[\%]}\end{array}$ & $\begin{array}{c}\text { RMSE } \\
{\left[1 / 10{ }^{\circ} \mathrm{C}\right]}\end{array}$ & \begin{tabular}{|c|} 
Additive \\
Konstante
\end{tabular} & $\begin{array}{l}\text { Gelände- } \\
\text { höhe }\end{array}$ & $\begin{array}{l}\text { QWKalt- } \\
\text { luftabfluß }\end{array}$ & TRA & $\begin{array}{l}\text { TRA*Gelände- } \\
\text { höhe }\end{array}$ \\
\hline Jan & 94,7 & 3,64 & 11,84 & $-0,0517$ & $-0,165$ & & \\
\hline $\mathrm{Feb}$ & 97,0 & 2,96 & 18,08 & $-0,0560$ & $-0,143$ & & \\
\hline Mär & 98,4 & 2,57 & 49,19 & $-0,0656$ & $-0,100$ & & \\
\hline Apr & 97,8 & 2,70 & 89,51 & $-0,0705$ & $-0,108$ & $-1,38$ & 0,0169 \\
\hline Mai & 97,6 & 3,01 & 136,84 & $-0,0674$ & $-0,122$ & $-13,56$ & 0,0224 \\
\hline Jun & 98,5 & 2,61 & 167,03 & $-0,0665$ & $-0,070$ & $-12,73$ & 0,0126 \\
\hline Jul & 95,6 & 4,15 & 182,67 & $-0,0675$ & $-0,196$ & 15,12 & 0,0429 \\
\hline Aug & 95,0 & 3,43 & 178,00 & $-0,0619$ & $-0,155$ & 3,31 & 0,0190 \\
\hline Sep & 98,3 & 2,66 & 145,71 & $-0,0593$ & $-0,092$ & $-14,67$ & 0,0124 \\
\hline Okt & 95,3 & 2,89 & 105,05 & $-0,0519$ & $-0,211$ & $-10,92$ & 0,0239 \\
\hline Nov & 94,9 & 3,15 & 57,13 & $-0,0578$ & $-0,139$ & $-9,26$ & 0,0305 \\
\hline Dez & 96,0 & 3,44 & 24,16 & $-0,0531$ & $-0,109$ & $-16,52$ & 0,0181 \\
\hline Jahr & 98,7 & 2,13 & 96,15 & $-0,0595$ & $-0,066$ & & \\
\hline
\end{tabular}

Die Koeffizienten der Dummy-Variable TRA weisen auf unterdurchschnittliche Temperaturen in den meisten Monaten der beiden Beobachtungsjahre des TRANSEKT-Datenkollektivs, 1994 und 1995, hin. Lediglich in den Sommermonaten Juli $\left(+1.5^{\circ} \mathrm{C}\right)$ und August $\left(+0.3{ }^{\circ} \mathrm{C}\right)$ wurden überdurchschnittlich hohe Temperaturen gemessen. 
Die negativen Regressionskoeffizienten der beiden räumlichen Variablen Geländehöhe und QWKaltluftabflu $\beta$ sind mit den in Kap. 3 gemachten Aussagen kongruent. Eine graphische Darstellung für den Januar vermittelt Abbildung 24. Deutlich zu erkennen ist die Abhängigkeit der Lufttemperatur von Geländehöhe und QWKaltluftabfluß. Allerdings sind auch die Lücken zwischen den beiden Stationen mit extremen Variablenwerten und dem Rest der Stationen zu sehen. Die Werte der durchschnittlichen Lufttemperatur im Januar dieser beiden Stationen „Einbeck“ und „Brocken“ werden jedoch ebenso gut durch das Modell abgebildet wie die der anderen Stationen. Das Modell ist also nicht nur durch die beiden „Ausreißer“ geprägt, es beschreibt einen Zusammenhang, der für die Gesamtheit der Stationen zu erkennen ist.

\section{Abbildung 24: Darstellung der Regionalisierungsfunktion für die durchschnittliche Lufttemperatur im Januar als Regression mit den räumlichen Variablen Geländehöhe und QWKaltluftabfluß}

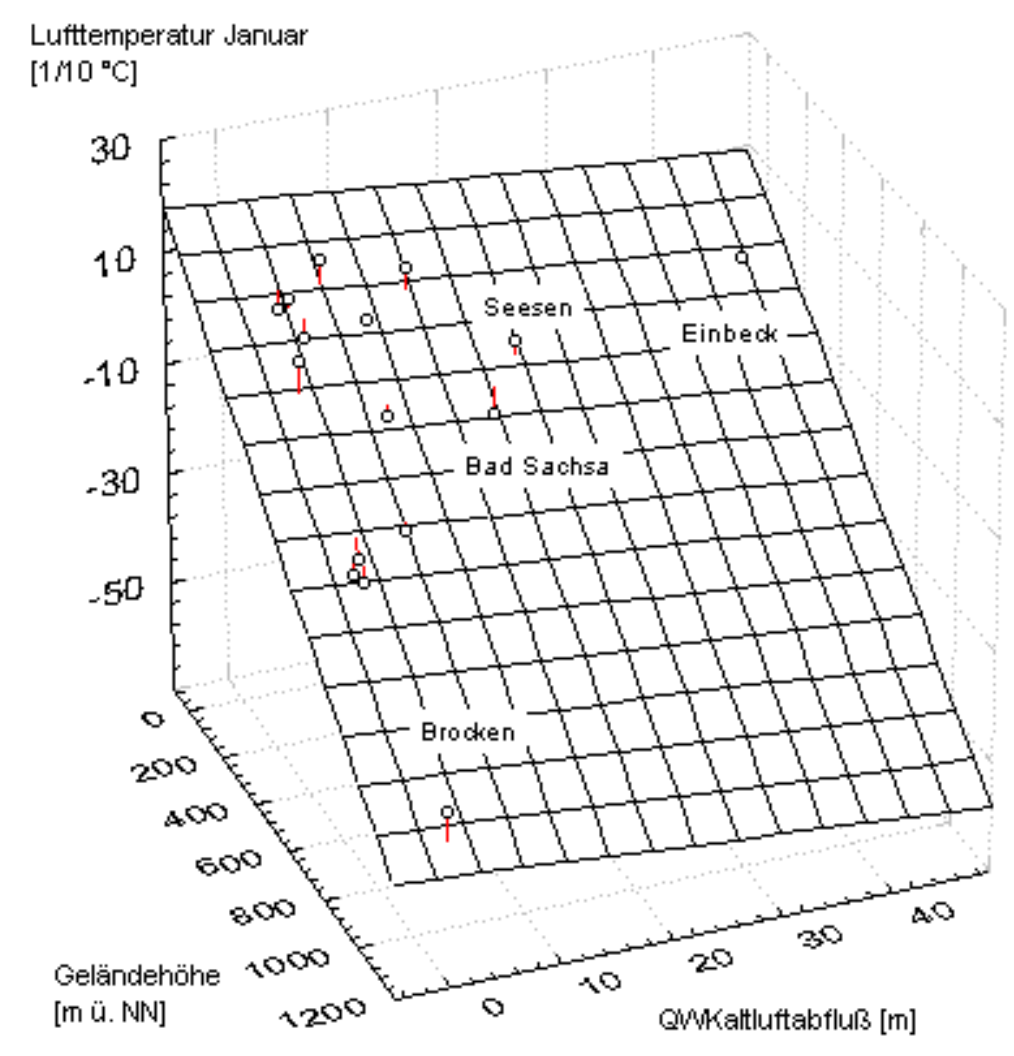

\subsubsection{Lufttemperatur im Tiefland}

In Tabelle 46 sind die Ergebnisse einer schrittweisen Regressionsanalyse aufgeführt. Die Modelle zeigen allgemein eine sehr gute Anpassung an die Daten. Das Modell 
für die mittlere Lufttemperatur im August zeigt jedoch ein Bestimmtheitsmaß von nur 65,1\%. Die durchschnittlichen Augusttemperaturen waren zudem die einzigen, die keinen bedeutenden Zusammenhang zu Variablen der geographischen Lage der Stationen (Kap. 3.2) erkennen ließen. Für die anderen Monate konnten der Hochwert (April und Mai), der Abstand zur Küste (Juni und Juli) und vor allem der Rechtswert (September bis März) einen großen Teil der räumlichen Variabilität erklären.

Die Anteile von Bebauung bzw. Gewässern an der Flächennutzung (Kap. 3.4.1) konnten zur weiteren Aufklärung der Temperaturvarianz beitragen. Vor allem die Regressionskoeffizienten für Gewässer lassen dabei einen jahreszeitlichen Trend erkennen. Dieser wird für die Monate März bis Juli, für die es zu keiner Modellbeteiligung des Gewässeranteils kam, durch negative Korrelationskoeffizienten nach Pearson zwischen Gewässer und Lufttemperatur bestätigt (ohne Abbildung). Der Zusammenhang zwischen Lufttemperatur und Gewässer wechselt also von einem wärmenden Einfluß im Herbst hin zu durchschnittlich niedrigeren Temperaturen im Frühling bei hohem Anteil an Gewässern.

Tabelle 46: Regressionskoeffizienten, Bestimmtheitsmaße $\left(\mathbf{R}^{2}\right)$ und Wurzel der mittleren quadrierten Residuen (RMSE) der Modelle für monatliche und jährliche mittlere Lufttemperatur im niedersächsischen Tiefland an 29 Stationen

\begin{tabular}{|c|c|c|c|c|c|c|c|}
\hline $\begin{array}{c}\text { Monat } \\
\text { /Jahr }\end{array}$ & $\begin{array}{c}\mathrm{R}^{2} \\
{[\%]}\end{array}$ & $\begin{array}{c}\text { RMSE } \\
{\left[1 / 10{ }^{\circ} \mathrm{C}\right]}\end{array}$ & & Geogr. Lage & Flächen & utzung & $\begin{array}{c}\text { Höhenabhängige } \\
\text { Variablen }\end{array}$ \\
\hline & & & $\begin{array}{c}\text { Additive } \\
\text { Konstante }\end{array}$ & $\begin{array}{c}\text { Rechtswert } \\
\text { (Sep-Mär, Jahr) } \\
\text { Hochwert } \\
\text { (Apr-Mai) } \\
\text { Abstand z. Küste } \\
\text { (Jun-Jul) }\end{array}$ & Bebauung & Gewässer & $\begin{array}{c}\text { expmin } \\
\text { (Apr bis Jul) } \\
\text { Lee-Effekt 25 } \\
\text { (Mär, Aug, Jahr) }\end{array}$ \\
\hline Jan & 88,3 & 2,01 & 236,24 & $-6,548 \mathrm{E}-05$ & & & \\
\hline $\mathrm{Feb}$ & 87,8 & 1,77 & 202,30 & $-5,485 \mathrm{E}-05$ & 7,819 & & \\
\hline Mär & 80,7 & 1,79 & 188,03 & $-4,384 \mathrm{E}-05$ & 8,282 & & 0,05009 \\
\hline Apr & 84,7 & 1,63 & 422,94 & $-5,914 \mathrm{E}-05$ & 7,467 & & $-0,13911$ \\
\hline Mai & 79,6 & 2,31 & 539,57 & $-7,094 \mathrm{E}-05$ & 9,472 & & $-0,14014$ \\
\hline Jun & 84,4 & 1,95 & 148,93 & $7,260 \mathrm{E}-05$ & 10,209 & & $-0,15570$ \\
\hline Jul & 83,1 & 1,56 & 163,75 & $5,015 \mathrm{E}-05$ & 9,763 & & $-0,12768$ \\
\hline Aug & 65,1 & 2,04 & 160,55 & & 10,031 & 8,608 & 0,04261 \\
\hline Sep & 79,0 & 2,16 & 200,30 & $-1,945 \mathrm{E}-05$ & 9,349 & 13,651 & \\
\hline Okt & 86,3 & 2,03 & 211,27 & $-3,369 \mathrm{E}-05$ & 8,915 & 13,773 & \\
\hline Nov & 82,6 & 2,29 & 183,44 & $-3,816 \mathrm{E}-05$ & & 11,934 & \\
\hline Dez & 87,1 & 2,10 & 201,37 & $-5,189 \mathrm{E}-05$ & & 8,152 & \\
\hline Jahr & 76,4 & 1,57 & 197,78 & $-3,257 \mathrm{E}-05$ & 7,859 & & 0,03467 \\
\hline
\end{tabular}


Als höhenabhängige Variablen konnten lediglich die Exponiertheit zum Umgebungsminimum (expmin, Kap. 3.3.5) für die Monate April bis Juli sowie der Lee-Effekt 25 (Kap. 3.3.3) für die Monate März und August und für die Jahresdurchschnittstemperatur einen bedeutsamen Anteil der Lufttemperaturvarianz aufklären. Daß gerade die Variable Lee-Effekt 25 im Jahresmodell integriert ist, kann nicht von den Ergebnissen für die Monatstemperaturen abgeleitet werden.

Einen Überblick über den Beitrag der einzelnen Variablen zur Varianzaufklärung bieten die folgenden Abbildungen. Abbildung 25 zeigt die Beiträge zum Bestimmtheitsmaß der lageabhängigen Variablen Rechtswert, Hochwert und Abstand z. Küste. Nur die Varianzen der durchschnittlichen Lufttemperatur im September, im Oktober und der Jahresmitteltemperatur können zu weniger als $40 \%$ alleine durch die Varianz einer dieser drei Variablen erklärt werden.

Die restlichen an den Modellen beteiligten Variablen werden in Abbildung 26 dargestellt. Besonders der jahreszeitlich wechselnde Einfluß der Landnutzungsvariablen Bebauung und Gewässer wird hier deutlich. Nur in den Frühlings- und Sommermonaten tragen auch höhenabhängige Variablen bedeutend zur Varianzaufklärung bei.

Während also im niedersächsischen Bergland der Einfluß der Geländehöhe von entscheidender Bedeutung für die Aufklärung der Varianz der Zielvariablen Lufttemperatur ist, sind im niedersächsischen Tiefland zumeist lageabhängige Variablen in besonderem Maß an der Modellierung der Lufttemperatur beteiligt. Zudem zeigen in einigen Monaten die Landnutzungsvariablen Gewässer und Bebauung ein großes Erklärungspotential. 
Abbildung 25: DWD Temperaturdatenkollektiv Tiefland: Beiträge zum Bestimmtheitsmaß der lageabhängigen Variablen

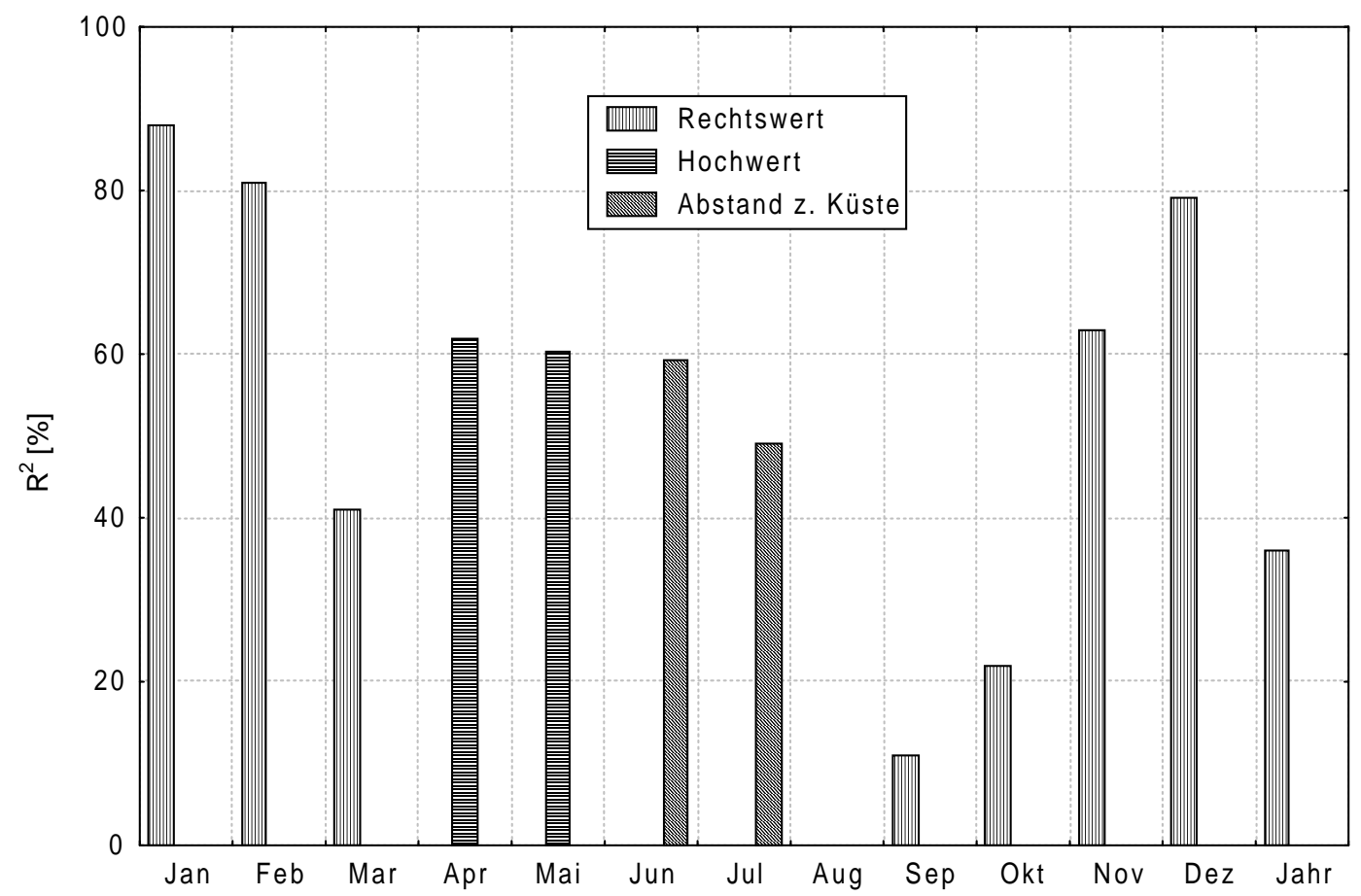

Abbildung 26: DWD Temperaturdatenkollektiv Tiefland: Beiträge zum Bestimmtheitsmaß der höhenabhängigen Variablen und der Variablen der Landnutzung

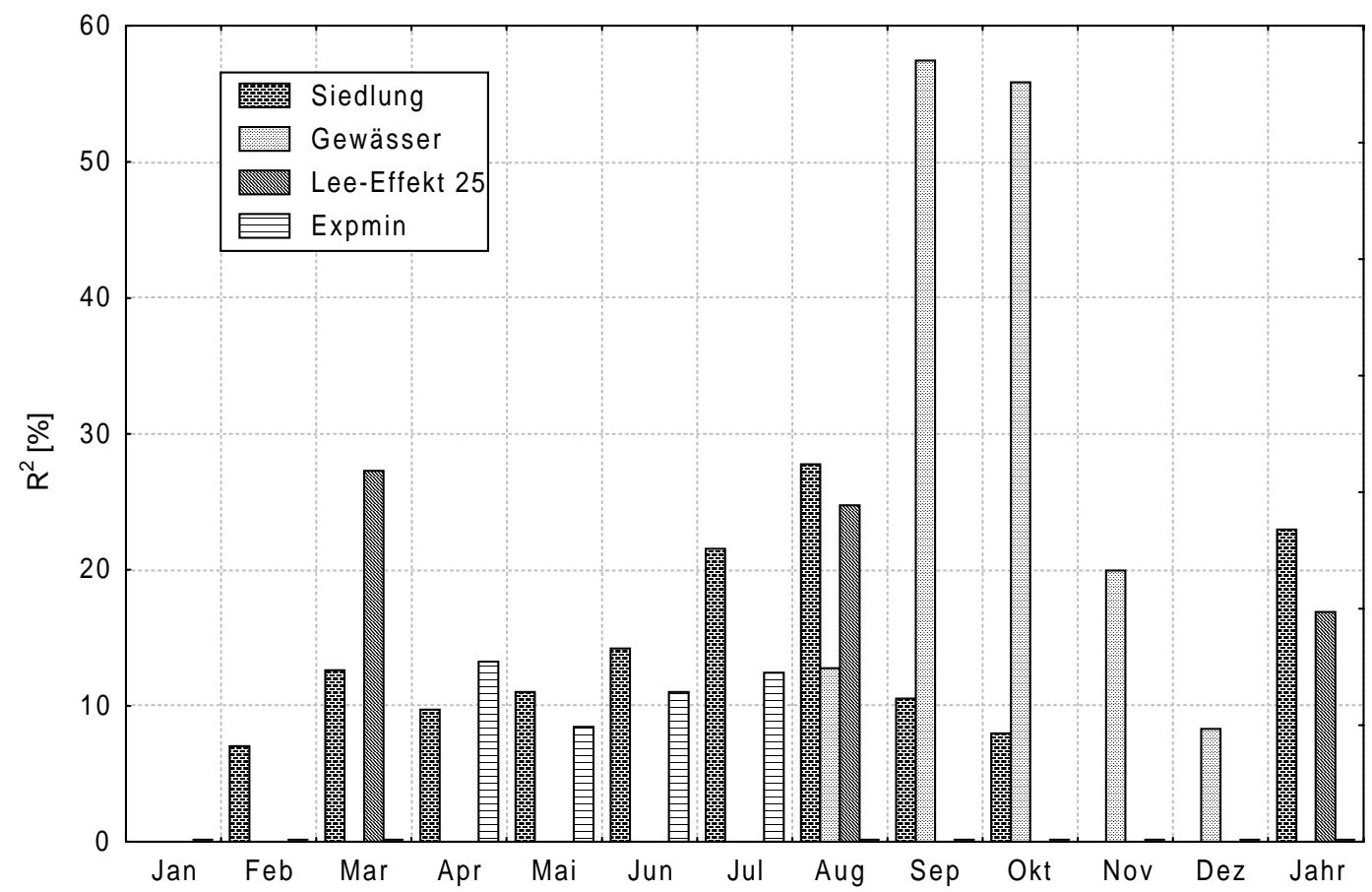




\subsubsection{Niederschlagssumme}

Im Falle der durchschnittlichen monatlichen und jährlichen Niederschlagssummen konnte im Anschluß an die schrittweise Regressionsanalyse eine geostatistische Analyse durchgeführt werden, da das Niederschlagmeßnetz des Deutschen Wetterdienstes (DWD) über ausreichend viele, räumlich günstig verteilte Stationen verfügt (Kap. 4.1.3). Für die Regionalisierung des Niederschlags im Bergland standen zusätzlich Daten der Harzwasserwerke (HWW) zur Verfügung. Diese konnten wegen gleicher Meßmethodik und gleichem Beobachtungszeitraum zur Ergänzung der Datenbasis genutzt werden.

Analog zu den Darstellungen zur Regionalisierung der Lufttemperatur (Kap. 5.1.1) soll auch für die Niederschlagssummen die Aufspaltung des Gesamtkollektivs in Bergland- und Tieflandkollektiv anhand der Präzision begründet werden. Um beurteilen zu können, ob und wie stark die Präzision der Modelle für Berg- und Tiefland gegenüber derjenigen der Modelle für gesamt Niedersachsen (Tabelle 47) verbessert ist, wurden die Summen der quadrierten Residuen der Regressionsmodelle und die Anzahl der in die Modelle integrierten räumlichen Variablen verglichen (Tabelle 48).

Tabelle 47: Regressionskoeffizienten, Bestimmtheitsmaße $\left(\mathbf{R}^{2}\right)$ und Wurzel der mittleren quadrierten Residuen (RMSE) der Modelle für monatliche und jährliche mittlere Niederschlagssummen in Niedersachsen (388 Stationen)

\begin{tabular}{|c|c|c|c|c|c|c|c|}
\hline $\begin{array}{c}\text { Monat/ } \\
\text { Jahr }\end{array}$ & $\begin{array}{c}\mathbf{R}^{2} \\
{[\%]}\end{array}$ & $\begin{array}{c}\text { RMSE } \\
{[1 / 10 ~ m m]}\end{array}$ & $\begin{array}{c}\text { Additive } \\
\text { Konstante }\end{array}$ & $\begin{array}{l}\text { Gelände- } \\
\text { höhe }\end{array}$ & Leewirbel & Luv & $\begin{array}{l}\text { Abstand } z \text {. } \\
\text { Küste }\end{array}$ \\
\hline Jan & 79,7 & 81,48 & 673,86 & 1,456 & 0,636 & & $-0,00179$ \\
\hline Feb & 83,2 & 53,82 & 438,52 & 1,009 & 0,391 & & $-0,00082$ \\
\hline Mär & 84,2 & 59,04 & 574,07 & 1,208 & 0,503 & & $-0,00130$ \\
\hline Apr & 78,9 & 52,46 & 468,78 & 0,747 & & & \\
\hline Mai & 69,2 & 53,94 & 572,00 & 0,596 & & & \\
\hline Jun & 72,0 & 63,55 & 714,98 & 0,751 & & & \\
\hline Jul & 74,0 & 58,61 & 794,12 & 0,869 & & 0,366 & $-0,00144$ \\
\hline Aug & 73,8 & 49,68 & 731,91 & 0,717 & & 0,290 & $-0,00094$ \\
\hline Sep & 75,2 & 58,00 & 701,63 & 0,890 & 0,424 & & $-0,00175$ \\
\hline Okt & 82,3 & 59,11 & 693,10 & 1,137 & 0,465 & & $-0,00225$ \\
\hline Nov & 82,3 & 73,54 & 784,32 & 1,472 & 0,633 & & $-0,00240$ \\
\hline Dez & 82,4 & 89,74 & 737,75 & 1,706 & 0,744 & & $-0,00183$ \\
\hline Jahr & 85,3 & 585,45 & 7946,69 & 12,732 & 5,504 & & $-0,01557$ \\
\hline
\end{tabular}


Die Modelle für das Gesamtdatenkollektiv des DWD werden mit vier räumlichen Variablen in unterschiedlicher Kombination gebildet. Die Variable mit dem höchsten Erklärungspotential in allen Modellen ist die Geländehöhe. Für die drei monatlichen Niederschlagssummen von April bis Juni wurde das Modell ausschließlich durch diese Variable gebildet. Alle anderen Modelle berücksichtigen zusätzlich den Abstand zur Küste und Leewirbel bzw. Luv (Juli und August).

Tabelle 48: Vergleich von Summen der quadrierten Residuen (SQR) für gemeinsame Modellierung (MOD: NDS) und für getrennte Modelle des Niederschlages (MOD: TIEF, bzw. MOD: BERG); in Klammern die Anzahl der Regressoren

\begin{tabular}{|c|c|c|c|c|c|c|c|c|c|}
\hline \multirow[b]{2}{*}{$\mathbf{N}$} & \multicolumn{3}{|c|}{ MOD: NDS } & \multicolumn{2}{|c|}{ Vorteil spez. Modell } & \multicolumn{4}{|c|}{ MOD: TIEF MOD: BERG } \\
\hline & $\begin{array}{c}\text { Gesamt } \\
388\end{array}$ & $\begin{array}{c}\text { Tiefland } \\
260\end{array}$ & $\begin{array}{c}\text { Bergland } \\
128\end{array}$ & $\begin{array}{c}\text { Tiefland } \\
260\end{array}$ & $\begin{array}{c}\text { Bergland } \\
128\end{array}$ & $\begin{array}{r}\text { Tieflaı } \\
260\end{array}$ & & $\begin{array}{c}\text { Berglan } \\
128\end{array}$ & \\
\hline Jan & 2549611 & 948449 & 1601162 & 323479 & -252641 & 624970 & (3) & 1853803 & \\
\hline Feb & 1112281 & 484147 & 628134 & 151828 & -72187 & 332319 & (2) & 700321 & (2) \\
\hline Mär & 1338295 & 613357 & 724938 & 283012 & -226675 & 330345 & (3) & 951613 & (2) \\
\hline Apr & 1062218 & 319070 & 743148 & 80892 & 266667 & 238178 & (2) & 476481 & (2) \\
\hline Mai & 1123240 & 577997 & & 80962 & 132041 & 497035 & (2) & 413202 & (2) \\
\hline Jun & 1558834 & 654407 & 904427 & 95907 & 416962 & 558500 & (2) & 487465 & (2) \\
\hline Jul & 1318951 & 629293 & 689658 & 4509 & -147071 & 624784 & (2) & 836729 & (2) \\
\hline Aug & 947684 & 543552 & 404132 & 34985 & 24404 & 508567 & (2) & 379728 & (3) \\
\hline Sep & 1291671 & 753216 & 538455 & -23176 & -120972 & 776392 & (2) & 659427 & (2) \\
\hline Okt & 1341550 & 779096 & 562454 & 181994 & 16887 & 597102 & (3) & 545567 & (2) \\
\hline Nov & 2076850 & 1031067 & 1045783 & 134533 & -190187 & 896534 & (3) & 1235970 & (2) \\
\hline Dez & 3092599 & 1092051 & 2000548 & 342172 & -273490 & 749879 & (3) & 2274038 & (2) \\
\hline Jahr & $131615853(3)$ & 52419802 & 79196051 & 8874803 & -19416888 & 435449 & (3) & 98612939 & (2) \\
\hline
\end{tabular}

In Bezug auf den Vergleich der Präzision von gemeinsamer und getrennter Modellierung zeigt sich ein ähnliches Bild wie für die Temperaturdaten, wenn auch auf den ersten Blick nicht ganz so eindeutig. So sind in neun Fällen Modelle für Teildatenkollektive schlechter angepaßt als das entsprechende Gesamtmodell (Tiefland: September, Bergland: November bis März, Juli, September und Jahr). Diese Modelle geringerer Präzision verwenden allerdings auch einen Regressor weniger als das entsprechende Gesamtmodell und sind damit einfacher. Alle anderen Teilkollektivmodelle sind von höherer Präzision als das entsprechende Gesamtmodell. Besonders bedeutungsvoll ist die höhere Präzision einiger Teilkollektivmodelle bei weniger Regressoren als im entsprechenden Gesamtmodell (Tiefland: Februar, Juli und August; Bergland: Oktober). Dieses Ergebnis und eine fast durchgehende Verbesserung der Präzision für das Tiefland unterstreichen die Plausibilität der Teilung des Datenkollektivs bzw. des Zielgebietes der Regionalisierung. 
Sowohl für das Tiefland als auch für das Bergland lagen ausreichend viele Meßstationen vor, um geostatistische Verfahren zur Verbesserung des Regionalisierungsergebnisses anzuwenden. Die Residuen der durch schrittweise Regression (ordinary least squares, OLS) gefundenen Modelle wurden als Basis für iterative Semivariogrammanpassungen genutzt. Das endgültige Regionalisierungsmodell sind GLS-Regressionsgleichungen mit zugehörigen Semivariogrammen.

\subsubsection{Niederschlagssumme im Bergland}

Zunächst sollen in diesem Kapitel die Ergebnisse der Regionalisierung anhand der reinen DWD-Berglanddaten dargestellt werden. Anschließend werden die Ergebnisse einer Analyse des um die HWW-Daten erweiterten Berglandkollektivs dargestellt. Mit der Verwendung des TRANSEKT-Datenkollektivs liegt ein zweites Beispiel für die Regionalisierung mit kombinierten Datenkollektiven vor. Probleme, die durch diese auf den Harz beschränkte Meßnetzverdichtung für die geostatistische Analyse entstehen, können durch den Vergleich mit einer ausschließlich auf DWD-Daten basierenden Regionalisierung verdeutlicht werden.

Das DWD-Datenkollektiv Bergland umfaßt 128 Stationen (vgl. 4.1.3.1). Die Modelle für die monatlichen und jährlichen durchschnittlichen Niederschlagssummen dieser Daten sind in Tabelle 49 zusammengefaßt.

Tabelle 49: Niederschlagssummen Bergland, DWD-Datenkollektiv (128 Stationen): Modellkoeffizienten und Parameter der Modellanpassung (Bestimmtheitsmaß $\mathbf{R}^{2}$ und rooted mean squared error RMSE) für monatliche und jährliche durchschnittliche Niederschlagssummen (1961 - 1990)

\begin{tabular}{|c|c|c|c|c|c|c|c|}
\hline $\begin{array}{c}\text { Monat/ } \\
\text { Jahr }\end{array}$ & $\begin{array}{c}\mathrm{R}^{2} \\
{[\%]}\end{array}$ & $\begin{array}{c}\text { RMSE } \\
{[1 / 10 \mathbf{~ m m}]}\end{array}$ & \begin{tabular}{|c|} 
Additive \\
Konstante
\end{tabular} & $\begin{array}{l}\text { Gelände- } \\
\text { höhe }\end{array}$ & Leewirbel & Luv & Hochwert \\
\hline Jan & 82,1 & 121,78 & 313,52 & 1,352 & 0,640 & & \\
\hline Feb & 85,9 & 74,85 & 263,83 & 0,974 & 0,407 & & \\
\hline Mär & 85,6 & 87,25 & 320,14 & 1,116 & 0,491 & & \\
\hline Apr & 84,7 & 61,74 & 413,68 & 0,759 & 0,343 & & \\
\hline Mai & 78,0 & 57,49 & 541,76 & 0,557 & & 0,199 & \\
\hline Jun & 85,3 & 62,45 & 644,81 & 0,735 & & 0,344 & \\
\hline Jul & 76,4 & 81,82 & 540,48 & 0,734 & & 0,313 & \\
\hline Aug & 85,5 & 55,34 & $-6949,56$ & 0,724 & & 0,332 & 0,00130 \\
\hline Sep & 77,9 & 72,63 & 398,76 & 0,708 & 0,333 & & \\
\hline Okt & 87,4 & 66,06 & 295,53 & 0,920 & 0,373 & & \\
\hline Nov & 85,2 & 99,44 & 352,77 & 1,250 & 0,550 & & \\
\hline Dez & 83,5 & 134,88 & 381,48 & 1,576 & 0,731 & & \\
\hline Jahr & 85,8 & 888,20 & 5059,06 & 11,391 & 5,157 & & \\
\hline
\end{tabular}


Die Anpassung der Modelle kann angesichts der Werte für $\mathrm{R}^{2}$ und RMSE als gut bezeichnet werden. Bei ähnlich hohen Werten für das Bestimmtheitsmaß wurden für die Wintermonate größere Werte für den RMSE ermittelt als in den Sommermonaten. Dies läßt sich durch die höhere Varianz der Zielvariablen in den Wintermonaten erklären (s. Tabelle 23, S. 76). Positiv fällt der relativ niedrige RMSE für das Junimodell auf, da in diesem Monat die höchsten Niederschlagssummen gemessen wurden. Insgesamt sind die Werte mit bis zu 13,4 mm für die monatlichen Modelle bzw. 88,8 mm für das Jahresmodell sehr gering im Verhältnis zu den Mittelwerten der Messungen (s. Tabelle 23) und zu den möglichen Meßfehlern (Tabelle 21, S. 74). Für die meisten Monatsmodelle wurde sogar ein RMSE unter $8 \mathrm{~mm}$ ermittelt.

Die Modelle werden durch vier verschiedene Variablen gebildet. In jedem Modell ist die Geländehöhe beteiligt, die auch in allen Modellen den größten Anteil an der Varianzaufklärung hat. Von September bis April werden die Modelle durch die räumliche Variable Leewirbel komplettiert. Den Platz dieses Regressors nimmt in den Monaten Mai bis August die Variable Luv ein, im August kommt es zusätzlich zur Einbeziehung des Hochwertes. Vor allem die Koeffizienten von Leewirbel zeigen einen saisonalen Effekt, der demjenigen der Niederschläge mit Maxima in Winter und Sommer (Kap. 4.1.3.1) entspricht. Hier nicht dargestellte Regressionsmodelle für die Monate Mai bis August unter Verwendung der Geländehöhe und der Variable Leewirbel bestätigen dies mit einem lokalen Maximum des Leewirbel-Koeffizienten im Juni- Modell (0,39; Juli:0,37).

Die Variable Luv bildet in den Monaten Mai bis August zusammen mit der Geländehöhe die Regressionsmodelle. Nur in diesen sommerlichen Monaten kann diese Variable in Bezug auf ihr Erklärungspotential die Variable Leewirbel übertreffen.

Die lageabhängige Variable Hochwert wurde ausschließlich im Augustmodell aufgenommen. Die schrittweise Regressionsanalyse zeigte für diesen Monat dadurch eine Verbesserung des Bestimmtheitsmaßes von $5,72 \%$ gegenüber einem entsprechenden Modell ohne diese Variable. Der Regressionskoeffizient für Hochwert erschien plausibel, weshalb die Variable nach den in Kap. 2.5 beschriebenen Regeln ins Modell aufgenommen wurde. 
Nach der Bestimmung der Regressionsmodelle wurden iterativ Semivariogramme an die Residuen der Regressionen angepaßt (s. Kap. 2.5). Die resultierenden GLS- und Semivariogrammodelle sind in Tabelle 50 zusammengestellt.

Tabelle 50: Niederschlagssummen Bergland, DWD-Datenkollektiv (128 Stationen): Modellkoeffizienten (generalisierte kleinste QuadrateSchätzung, GLS) und Parameter der iterativ angepaßten sphärischen Semivariogramme

\begin{tabular}{l|rrrll|rrr}
\hline $\begin{array}{c}\text { Monat/ } \\
\text { Jahr }\end{array}$ & \multicolumn{3}{|c|}{ GLS-Modell } & \multicolumn{3}{c}{ Semivariogramm } \\
\cline { 2 - 9 } & $\begin{array}{c}\text { Additive } \\
\text { Konstante }\end{array}$ & $\begin{array}{c}\text { Gelände- } \\
\text { höhe }\end{array}$ & $\begin{array}{c}\text { Lee- } \\
\text { wirbel }\end{array}$ & Luv & Hochwert & Nugget & Sill & Range \\
\hline Jan & 339,84 & 1,245 & 0,453 & & & 7680 & 7580 & 52000 \\
Feb & 281,42 & 0,899 & 0,302 & & & 3190 & 2450 & 50000 \\
Mär & 319,59 & 1,113 & 0,435 & & & 4700 & 2800 & 80000 \\
Apr & 411,78 & 0,737 & 0,287 & & & 2150 & 1650 & 65000 \\
Mai & 558,19 & 0,492 & & 0,117 & & 600 & 2890 & 32000 \\
Jun & 688,59 & 0,576 & & 0,197 & & 650 & 3900 & 27300 \\
Jul & 581,71 & 0,621 & & 0,136 & & 0 & 7780 & 22500 \\
Aug & $-5135,80$ & 0,617 & & 0,208 & 0,000991 & 700 & 2655 & 21800 \\
Sep & 416,65 & 0,646 & 0,206 & & & 1530 & 3950 & 28800 \\
Okt & 319,61 & 0,822 & 0,264 & & & 2200 & 2215 & 33300 \\
Nov & 385,05 & 1,109 & 0,375 & & & 4000 & 6280 & 47000 \\
Dez & 402,98 & 1,492 & 0,552 & & & 10900 & 7300 & 58000 \\
\hline Jahr & 5306,32 & 10,192 & 3,548 & & & 361000 & 456500 & 45000 \\
\hline
\end{tabular}

Im Vergleich zu den Regressionskoeffizienten der OLS-Schätzungen sind - die Werte der additiven Konstante ausgenommen - alle Beträge der Regressionskoeffizienten der GLS-Schätzung niedriger. Hier kommt zum Ausdruck, daß ein Teil der Zielvariablenvarianz nun durch ihre räumliche Autokorrelation erklärt wird. Dieser Teil wird vom OLS-Modell noch auf die Varianz der räumlichen Variablen zurückgeführt. Der Einfluß der einzelnen Variablen und somit ihre Koeffizienten sind im GLS-Modell daher geringer als im OLS-Modell.

Die angepaßten Semivariogramme sind durchweg sphärisch modelliert worden. Ihre Form wird durch die drei Parameter Nugget, Sill und Range beschrieben. Ein Beispiel für ein solches Semivariogramm ist in Abbildung 27 dargestellt. An das punkthaft dargestellte empirische Semivariogramm wurde ein theoretisches angepaßt. Die Parameter aller Bergland-Semivariogramme zeigen vor allem den Nugget betreffend einen saisonalen Effekt mit Minimum im Sommer und Maximum im Winter, während die Werte des Sill analog zu den durchschnittlichen Werten der Niederschlagssummen mit lokalen Maxima im Winter und im Hochsommer verlaufen (vgl. Tabelle 23, S. 76). 
Abbildung 27: Iterativ angepaßtes Semivariogramm zur Beschreibung der räumlichen Autokorrelation der langjährigen durchschnittlichen Niederschlagssumme im Oktober für das niedersächsische Bergland (128 DWD-Stationen); Nugget 2200, Sill 2215, Range 33300

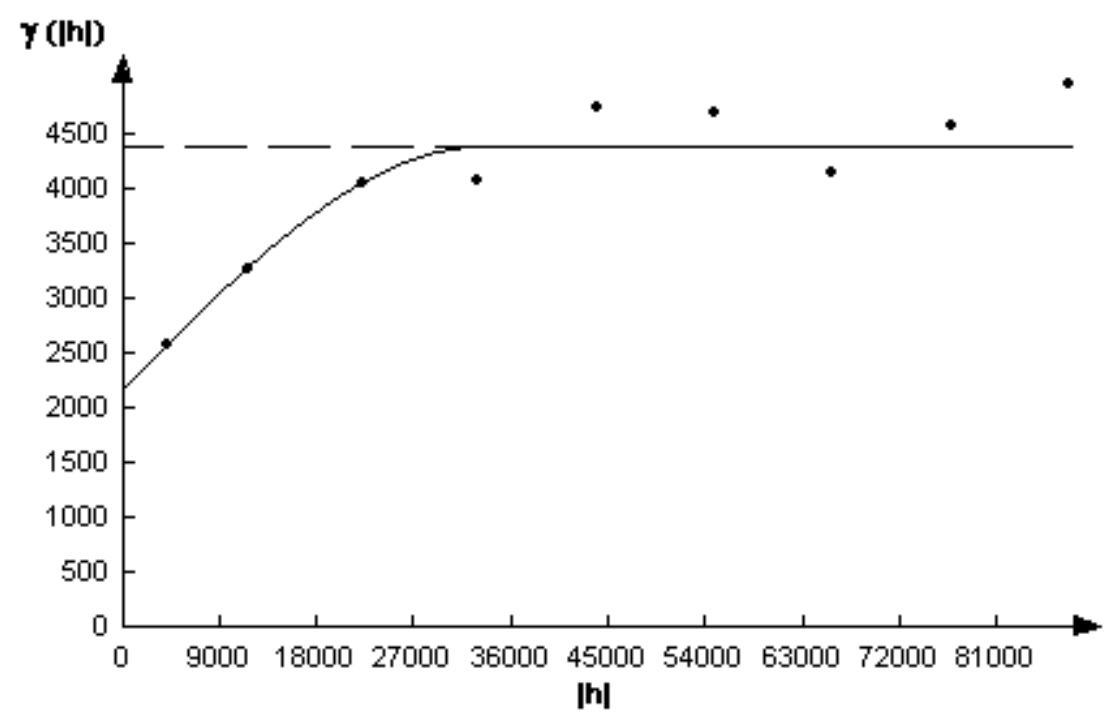

Nicht alle empirischen Semivariogramme waren so typisch sphärischer Gestalt wie das oben dargestellte. Abweichungen waren jedoch nicht so gravierend, als daß ein anderes Modell angepaßt werden mußte. Auch eine Anisotropie, eine Richtungsabhängigkeit der räumlichen Autokorrelation, wurde nicht gefunden. Da die Anpassung gutachterlich erfolgte, ist eine Einflußnahme des Bearbeiters nicht ausgeschlossen. Die Auswirkungen von verschiedenen Anpassungen theoretischer Semivariogramme auf die Koeffizienten der GLS- Regression und die Ergebnisse der anschließenden Kreuzvalidierung wurden jedoch exemplarisch untersucht und als nicht bedeutend eingestuft.

Eine Kreuzvalidierung kann Ergebnisse zur Quantifikation der Präzision des Krigings liefern. Die in Kap. 2.3 auf Seite 14 beschriebenen Standardabweichungen der Differenzen aus Kreuzvalidierungs-Vorhersage und Meßwert sind für die Berglandmodelle in Tabelle 51 in Spalte (3) aufgeführt. Mit diesem Wert wird die Standardabweichung der OLS-Residuen - ohne Berücksichtigung der räumlichen Autokorrelation - in Spalte (2) verglichen. Zur Veranschaulichung der Verbesserung durch Berücksichtigung der räumlichen Autokorrelation wurde zudem die Differenz aus den OLS-Standardabweichungen und den Kreuzvalidierungs-Standardabweichungen berechnet und in Spalte (4) hinzugefügt. Diese Differenz wurde in Spalte (5) im Verhältnis zu den OLS-Standardabweichungen dargestellt. 
Tabelle 51: Niederschlagssummen, DWD Berglanddatenkollektiv (128 Stationen): Ergebnis der Kreuzvalidierung (Spalte 3) und Vergleich mit der Modellanpassung nach Regression der Methode der gewöhnlichen kleinsten Quadrate (OLS; Spalte 2)

\begin{tabular}{|c|c|c|c|c|}
\hline $\begin{array}{c}\text { Monat/ } \\
\text { Jahr } \\
\text { (1) } \\
\end{array}$ & $\begin{array}{c}\text { STD } \\
\text { residual OLS } \\
{[1 / 10 ~ m m]} \\
(2) \\
\end{array}$ & $\begin{array}{c}\text { STD } \\
\mathbf{Z}^{*}-\mathrm{Z} \\
{[1 / 10 \mathrm{~mm}]} \\
(3) \\
\end{array}$ & $\begin{array}{c}\text { Differenz } \\
(2)-(3) \\
{[1 / 10 \mathrm{~mm}]} \\
(4) \\
\end{array}$ & $\begin{array}{c}\text { Relative Differenz } \\
(4) /(2) * 100 \\
{[\%]} \\
(5)\end{array}$ \\
\hline Jan & 120,8 & 84,1 & 36,8 & 30,4 \\
\hline Feb & 74,3 & 55,1 & 19,1 & 25,8 \\
\hline Mär & 86,6 & 68,8 & 17,8 & 20,5 \\
\hline Apr & 61,3 & 46,0 & 15,2 & 24,9 \\
\hline Mai & 57,0 & 35,1 & 21,9 & 38,5 \\
\hline Jun & 62,0 & 48,5 & 13,4 & 21,6 \\
\hline Jul & 81,2 & 48,9 & 32,3 & 39,8 \\
\hline Aug & 54,7 & 39,5 & 15,1 & 27,7 \\
\hline Sep & 72,1 & 42,7 & 29,3 & 40,7 \\
\hline Okt & 65,5 & 48,7 & 16,9 & 25,8 \\
\hline Nov & 98,7 & 70,1 & 28,5 & 28,9 \\
\hline Dez & 133,8 & 99,2 & 34,6 & 25,8 \\
\hline Jahr & 881,2 & 603,9 & 277,3 & 31,5 \\
\hline
\end{tabular}

Die Kreuzvalidierung weist im Verhältnis zu den OLS-Standardabweichungen eine Reduktion des Modellfehlers um 20,5 \% bis 40,7 \% auf. In absoluten Werten handelt es sich dabei um 1,5 mm bis 3,7 mm für die Monatsmodelle und um 27,7 mm für das Jahresmodell. Der Aufwand zur Ermittlung der geostatistischen Modelle ist nicht unerheblich, hält sich aber bei geeigneter Datenbasis sowie der entsprechenden Softund Hardware in Grenzen. Im Verhältnis zum Aufwand, der nötig ist, eine geeignete Datenbasis für die Regressionsanalyse aufzubauen, fällt dies bei der Auswahl der besten Methode nicht ins Gewicht (Kap. 6.1.2.1).

Wie in Kap. 4.1.1 erwähnt, konnte zusätzlich zu dem DWD-Datenkollektiv auf ein weiteres, das der Harzwasserwerke (HWW), zurückgegriffen werden. Da sämtliche Stationen des HWW-Datenkollektivs im Harz oder nahe zum Harz gelegen sind, wurden die Daten zusammen mit den Berglanddaten des DWD analysiert. Eine stufenweise Regressionsanalyse ergab die in Tabelle 52 zusammengestellten OLSModelle für die monatlichen und jährlichen Niederschlagssummen im niedersächsischen Bergland. 
Tabelle 52: Niederschlagssummen Bergland, DWD/HWW-Datenkollektiv (193 Stationen): Modellkoeffizienten und Parameter der Modellanpassung (Bestimmtheitsmaß $\mathbf{R}^{2}$ und rooted mean squared error RMSE) für monatliche und jährliche Niederschlagssummen (1961 - 1990)

\begin{tabular}{l|cc|cccc}
\hline $\begin{array}{c}\text { Monat/ } \\
\text { Jahr }\end{array}$ & $\begin{array}{c}\mathbf{R}^{2} \\
{[\%]}\end{array}$ & $\begin{array}{c}\text { RMSE } \\
{[\mathbf{1} / \mathbf{1 0} \mathbf{~ m m}]}\end{array}$ & $\begin{array}{c}\text { Additive } \\
\text { Konstante }\end{array}$ & Geländehöhe & Leewirbel & Lee-Effekt 50 \\
\hline Jan & 85,2 & 134,87 & 324,41 & 1,320 & 0,562 & \\
Feb & 88,5 & 83,47 & 269,09 & 0,952 & 0,372 & \\
Mär & 89,2 & 93,62 & 325,28 & 1,104 & 0,442 & \\
Apr & 89,1 & 65,26 & 416,52 & 0,768 & 0,304 & \\
Mai & 78,1 & 68,35 & 575,97 & 0,578 & & \\
Jun & 84,0 & 76,46 & 671,24 & 0,719 & 0,285 & $-0,668$ \\
Jul & 78,9 & 97,00 & 907,28 & & & $-0,529$ \\
Aug & 76,9 & 81,55 & 864,42 & & & $-0,605$ \\
Sep & 78,3 & 89,26 & 727,04 & & & \\
Okt & 88,7 & 77,46 & 301,59 & 0,902 & 0,329 & \\
Nov & 87,4 & 113,16 & 357,69 & 1,228 & 0,484 & \\
Dez & 86,7 & 153,72 & 380,85 & 1,608 & 0,652 & \\
\hline Jahr & 88,6 & 983,07 & 5131,55 & 11,304 & 4,382 & \\
\hline
\end{tabular}

Wie bereits für die Modelle des reinen DWD-Datenkollektivs beobachtet (vgl. Tabelle 49, S. 117), werden auch für das integrierte DWD/HWW-Datenkollektiv die meisten Regressionsmodelle mit den beiden räumlichen Variablen Geländehöhe und Leewirbel gebildet. Die Variablen Luv und Hochwert sind nicht in den Modellen des integrierten Datenkollektivs vertreten. Im Mai kann kein zusätzlicher Regressor zur Geländehöhe das Bestimmtheitsmaß des Modells um mindestens $5 \%$ verbessern. In den Monaten Juli bis September ist Lee-Effekt 50 die räumliche Variable mit dem höchsten Erklärungspotential. Die Geländehöhe wird nach den in Kap. 2.5 beschriebenen Regeln nicht zur Modellbildung herangezogen.

Die Bestimmtheitsmaße der DWD/HWW-Modelle sind bis auf Juni und August größer als die der korrespondierenden DWD-Modelle. Der RMSE der DWD/HWWModelle ist allerdings für alle Monatsmodelle und das Jahresmodell ebenfalls größer als für die DWD-Modelle. Die Kombination dieser beiden Beobachtungen ist Resultat der höheren Gesamtvarianz innerhalb des integrierten Datenkollektivs.

Für das integrierte DWD/HWW-Datenkollektiv konnte die Variable Luv keine ähnlich hohe Bedeutung für die Erklärung der räumlichen Varianz des Niederschlages erreichen wie im Fall der DWD-Modelle. Wäre der durch Luv beschriebene Luv-Effekt im Harz nicht ebenso wirksam wie im restlichen Bergland, so müßten auch Modelle, die ausschließlich für die DWD-Stationen im Harz angepaßt werden, diese Variable nicht enthalten. Dies ist jedoch nicht der Fall. Sowohl die Monatsmodelle für Februar und für April bis September als auch das 
Jahresmodell beinhalten nach schrittweiser Regression und den in Kap. 2.5, S. 21 beschriebenen Bedingungen - Erhöhung des Bestimmtheitsmaßes um $5 \%$ und Plausibilität - die räumliche Variable Luv neben der Geländehöhe (s. Anhang 9). Die entsprechenden Modelle für das HWW-Datenkollektiv (s. Anhang 10) bestätigen das niedrige Erklärungspotential der Variable Luv für die HWW-Daten. Der einfache Korrelationskoeffizient nach Pearson zeigt für das HWW-Datenkollektiv sogar negative Werte, was der Interpretation des Luv-Indexes widerspricht. Mögliche Gründe für solch unterschiedliche Aussagen der beiden Datenkollektive werden in Kap. 6.1.2.1 diskutiert.

Die Residuen der DWD/HWW-Modelle zeigen durchweg eine schwächere räumliche Autokorrelation als die Residuen der DWD-Modelle. Zudem lassen sie Abweichungen von der angenommenen Stationarität erkennen. Eine Modellierung wird durch die im Vergleich zur Analyse der DWD-Modelle recht hohen Varianz der Residuen mit geringem Abstand (s. Abbildung 28) erschwert, für einige Monate sogar unmöglich gemacht. Im Vergleich mit dem in Abbildung 27 dargestellten beispielhaften Semivariogramm für das DWD-Datenkollektiv ist die Semivarianz vor allem der kürzesten Distanzen sehr viel größer. Inwiefern dies durch die Verknüpfung der beiden Datenkollektive erklärt werden kann, wird in Kap. 6.1.2.1 diskutiert.

\section{Abbildung 28: Empirisches Semivariogramm zur Beschreibung der räumlichen Autokorrelation der Residuen der langjährigen durchschnittlichen Niederschlagssumme im Oktober für das DWD/HWW-Datenkollektiv}

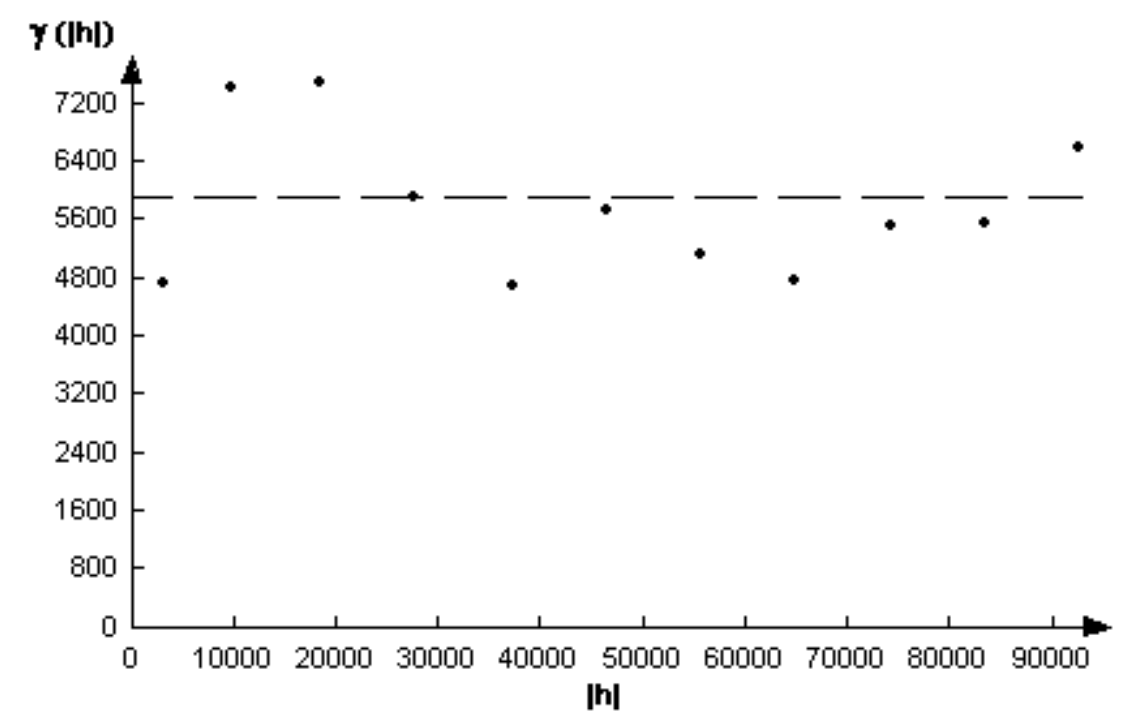


Für die Monate Januar, Februar, Mai, Juli, August, September, November und Dezember konnte die räumliche Autokorrelation durch Semivariogramme beschrieben werden. Diese sind zusammen mit den Koeffizienten der GLS-Modelle in Tabelle 53 dargestellt.

Tabelle 53: Niederschlagssummen Bergland, DWD/HWW-Datenkollektiv (193 Stationen): GLS-Regressionskoeffizienten und Parameter der iterativ angepaßten sphärischen Semivariogramme

\begin{tabular}{l|rrrr|rrr}
\hline \multirow{2}{*}{$\begin{array}{c}\text { Monat/ } \\
\text { Jahr }\end{array}$} & \multicolumn{3}{|c|}{ GLS-Modell } & \multicolumn{3}{c}{ Semivariogramm } \\
\cline { 2 - 7 } & Add.Konst. & $\begin{array}{c}\text { Gelände- } \\
\text { höhe }\end{array}$ & Leewirbel & Lee-Effekt 50 & Nugget & Sill & Range \\
\hline Jan & 377,8 & 1,1052 & 0,4575 & & 4480 & 15120 & 9300 \\
Feb & 294,5 & 0,8527 & 0,3202 & & 2850 & 4450 & 9000 \\
Mai & 598,7 & 0,4058 & & & 2375 & 2640 & 18000 \\
Jul & 790,6 & & & $-0,3769$ & 4200 & 8300 & 45550 \\
Aug & 762,0 & & & $-0,2778$ & 2400 & 7600 & 48650 \\
Sep & 622,9 & & & $-0,3278$ & 3600 & 7690 & 44640 \\
Nov & 383,1 & 1,1470 & 0,4356 & & 10200 & 2800 & 78000 \\
Dez & 433,2 & 1,3569 & 0,5194 & & 18000 & 6860 & 52100 \\
\hline
\end{tabular}

Die Modelle sind vor allem in Bezug auf den Range der Semivariogramme sehr unterschiedlich. Die Semivariogramme für Januar, Februar und Mai zeigen einen kleinen Range als anderen Modelle.

Abbildung 29: Iterativ angepaßtes Semivariogramm zur Beschreibung der räumlichen Autokorrelation der Residuen der langjährigen durchschnittlichen Niederschlagssumme im September für das niedersächsische Bergland (193 DWD/HWW-Stationen); Nugget 3600, Sill 7690, Range 44640

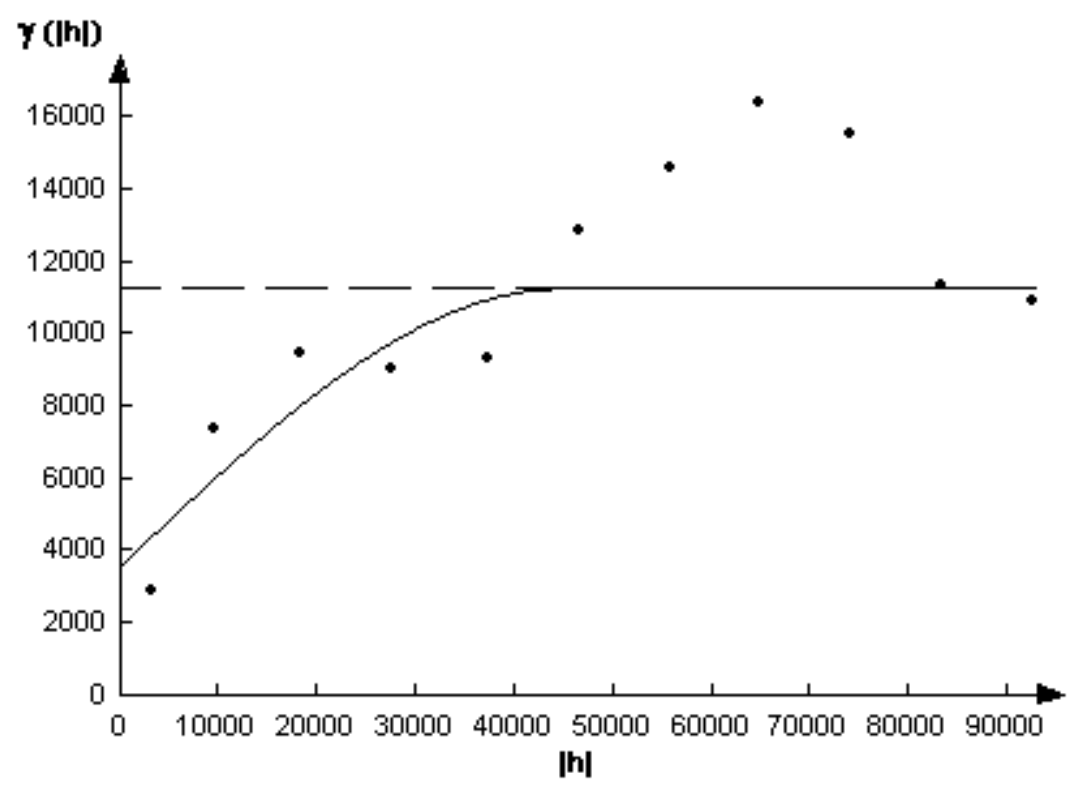


Die Verbesserung der Anpassung an die Daten wurde über Kreuzvalidierungen quantifiziert. Die Ergebnisse werden in Tabelle 54 dargestellt:

Tabelle 54: Niederschlagssummen, DWD/HWW-Datenkollektiv (193 Stationen): Ergebnis der Kreuzvalidierung (Spalte 3) und Vergleich mit der Modellanpassung nach Regression der Methode der gewöhnlichen kleinsten Quadrate (OLS; Spalte 2)

\begin{tabular}{|c|c|c|c|c|}
\hline $\begin{array}{c}\begin{array}{c}\text { Monat/ } \\
\text { Jahr }\end{array} \\
\text { (1) }\end{array}$ & $\begin{array}{c}\text { STD } \\
\text { residual OLS } \\
{[1 / 10 \mathrm{~mm}]} \\
(2)\end{array}$ & $\begin{array}{c}\text { STD } \\
Z^{*}-\mathbf{Z} \\
{[1 / 10 \mathrm{~mm}]} \\
(3)\end{array}$ & $\begin{array}{c}\text { Differenz } \\
(2)-(3) \\
{[1 / 10 \mathrm{~mm}]} \\
(4)\end{array}$ & $\begin{array}{c}\text { Relative Differenz } \\
\text { (4) / (2) *100 } \\
{[\%]} \\
(5)\end{array}$ \\
\hline Jan & 134,2 & 89,0 & 45,2 & 33,7 \\
\hline Feb & 83,0 & 60,8 & 22,3 & 26,8 \\
\hline Mär & 93,1 & & & \\
\hline Apr & 64,9 & & & \\
\hline Mai & 68,2 & 45,5 & 22,7 & 33,3 \\
\hline Jun & 90,4 & & & \\
\hline Jul & 96,7 & 53,4 & 43,3 & 44,8 \\
\hline Aug & 81,3 & 47,4 & 33,9 & 41,7 \\
\hline Sep & 89,0 & 47,3 & 41,7 & 46,8 \\
\hline Okt & 77,1 & & & \\
\hline Nov & 112,6 & 81,3 & 31,3 & 27,8 \\
\hline Dez & 152,9 & 97,3 & 55,6 & 36,3 \\
\hline Jahr & 977,9 & & & \\
\hline
\end{tabular}

Die Kreuzvalidierungen zeigen eine Verbesserung der Genauigkeit um $27 \%$ bis $47 \%$ im Vergleich zu den Standardabweichungen der OLS-Regressionsresiduen. Die drei stärksten relativen Verbesserungen wurden für die Monate Juli bis September ermittelt. In diesen Monaten waren durch schrittweise lineare Regression Modelle durch die Variable Lee-Effekt 50 gebildet worden. Für sie war die Ermittlung und Beschreibung räumlicher Autokorrelation zudem am einfachsten und deutlichsten möglich.

\subsubsection{Niederschlagssumme im Tiefland}

Das DWD Tieflanddatenkollektiv für die langjährigen durchschnittlichen Niederschlagssummen bestand aus 260 Datensätzen. Die durch schrittweise multiple lineare Regression (Kap. 2.5) ermittelten Modelle für die durchschnittlichen monatlichen und jährlichen Niederschlagssummen sind in Tabelle 55 zusammengestellt. Nachfolgend werden zudem die Ergebnisse der geostatistischen Analyse der Daten bzw. Regressionsresiduen vorgestellt. 
Tabelle 55: Niederschlagssummen Tiefland, DWD-Datenkollektiv (260 Stationen): Modellkoeffizienten und Parameter der Modellanpassung (Bestimmtheitsmaß $\mathbf{R}^{2}$ und rooted mean squared error RMSE) für monatliche und jährliche Niederschlagssummen (1961 - 1990)

\begin{tabular}{l|cc|rccc}
\hline $\begin{array}{c}\text { Monat/ } \\
\text { Jahr }\end{array}$ & $\begin{array}{r}\mathbf{R}^{\mathbf{2}} \\
{[\%]}\end{array}$ & $\begin{array}{c}\text { RMSE } \\
{[\mathbf{1} / \mathbf{1 0} \mathbf{~ m m}]}\end{array}$ & $\begin{array}{c}\text { Additive } \\
\text { Konstante }\end{array}$ & $\begin{array}{c}\text { Gelände- } \\
\text { höhe }\end{array}$ & Rechtswert & $\begin{array}{c}\text { Abstand z. } \\
\text { Küste }\end{array}$ \\
\hline Jan & 58,4 & 49,41 & 2348,51 & 1,978 & $-4,96 \mathrm{E}-04$ & $-9,54 \mathrm{E}-04$ \\
Feb & 47,3 & 35,96 & 1730,70 & 1,164 & $-3,84 \mathrm{E}-04$ & \\
Mär & 65,6 & 35,92 & 2327,69 & 1,586 & $-5,17 \mathrm{E}-04$ & $-5,65 \mathrm{E}-04$ \\
Apr & 39,7 & 30,44 & 1311,52 & 0,901 & $-2,42 \mathrm{E}-04$ & \\
Mai & 22,9 & 43,98 & 1446,24 & 0,851 & $-2,53 \mathrm{E}-04$ & \\
Jun & 21,2 & 46,62 & 1618,34 & 0,850 & $-2,59 \mathrm{E}-04$ & \\
Jul & 55,3 & 49,31 & 801,51 & 0,848 & & $-1,44 \mathrm{E}-03$ \\
Aug & 29,9 & 44,48 & 725,38 & 0,989 & & $-8,55 \mathrm{E}-04$ \\
Sep & 64,7 & 54,96 & 710,26 & 1,434 & & $-2,04 \mathrm{E}-03$ \\
Okt & 81,4 & 48,30 & 2162,01 & 1,273 & $-4,24 \mathrm{E}-04$ & $-2,08 \mathrm{E}-03$ \\
Nov & 72,8 & 59,18 & 2290,01 & 1,909 & $-4,37 \mathrm{E}-04$ & $-2,14 \mathrm{E}-03$ \\
Dez & 60,7 & 54,12 & 2732,39 & 2,282 & $-5,87 \mathrm{E}-04$ & $-1,05 \mathrm{E}-03$ \\
\hline Jahr & 65,1 & 412,43 & 19560,00 & 16,836 & $-3,40 \mathrm{E}-03$ & $-1,195 \mathrm{E}-02$ \\
\hline
\end{tabular}

Die Bestimmtheitsmaße der Modelle zeigen, daß im Vergleich zum niedersächsischen Bergland nur ein sehr viel geringerer Teil der Niederschlagsvarianz im Tiefland auf die Regressoren zurückgeführt werden konnte. Die niedrigsten Bestimmtheitsmaße weisen die Modelle der Monate von April bis August auf. Immerhin 81,4\% erreicht das Modell für den Monat Oktober. Die monatlichen Modelle zeigen dabei durchschnittliche Fehler (RMSE) von $3 \mathrm{~mm}$ bis 6,5 mm und liegen damit unter denen der Berglandmodelle und bis auf den März stets unter $10 \%$ der Stationsmittelwerte (vgl. Tabelle 28, S. 80).

Die höchsten Bestimmtheitsmaße wurden für diejenigen Modelle ermittelt, die durch die drei räumlichen Variablen Geländehöhe, Rechtswert und Abstand zur Küste gebildet werden. Die Variable Geländehöhe trägt dabei nicht immer- wie im Fall der Berglandmodelle - den größten Teil zur Erklärung der Zielvariablenvarianz bei (s. Abbildung 30), ist aber der einzige konsistente Bestandteil der Modelle, die einen jährlichen Trend für die beiden anderen Variablen zeigen. 
Abbildung 30: Niederschlagssummen, DWD-Tieflanddatenkollektiv: Beiträge zum Bestimmtheitsmaß der räumlichen Variablen der Modelle

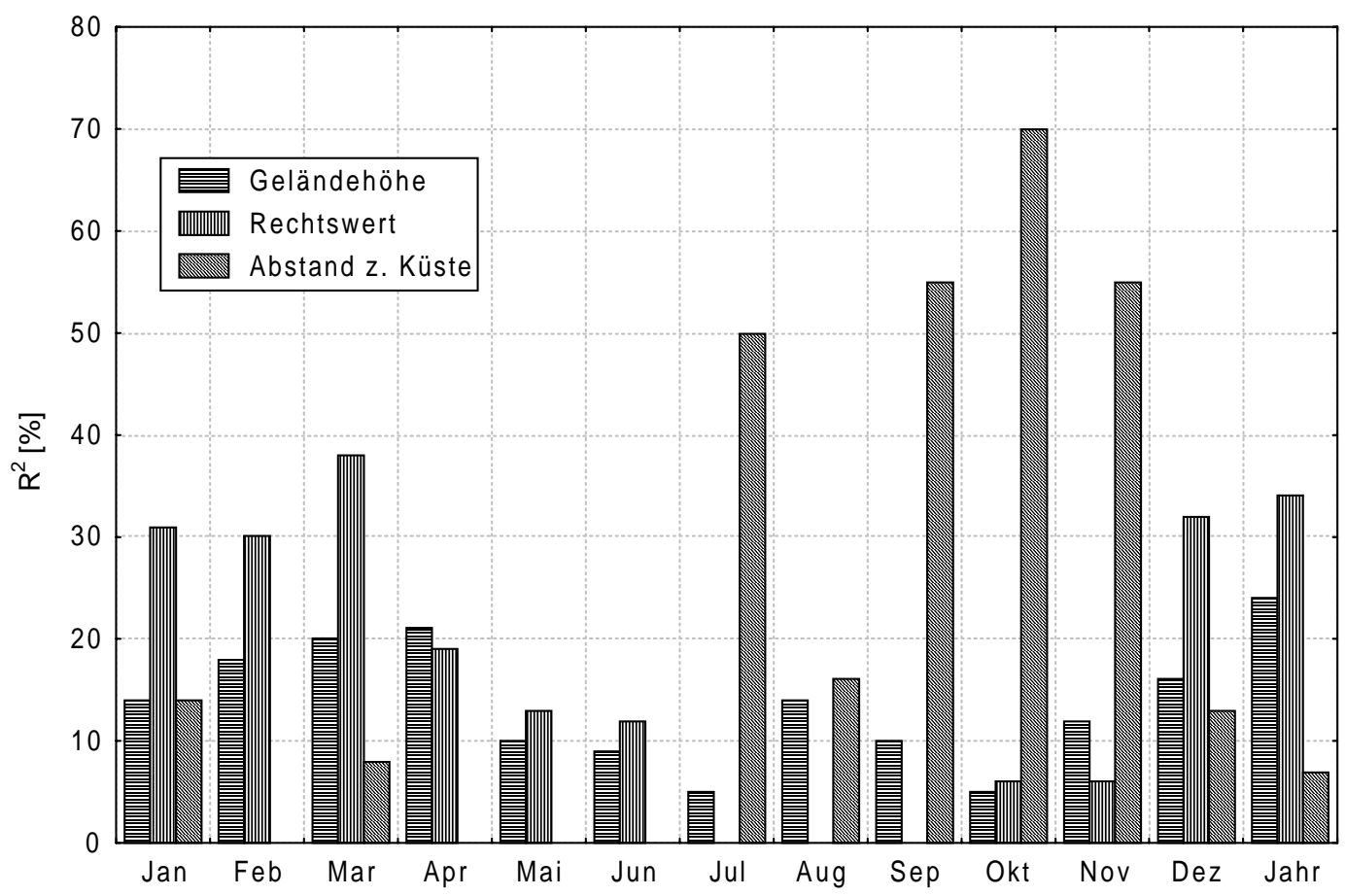

Die durch die Modelle der schrittweisen Regression berechneten Residuen wurden analog zu der Vorgehensweise im Bergland als Basis für eine iterative Semivariogrammanpassung verwendet (Kap. 2.5). Die so entstandenen GLS-Modelle und Semivariogramme sind in Tabelle 56 zusammengefaßt.

Tabelle 56: Niederschlagssummen, DWD-Tieflanddatenkollektiv: Modellkoeffizienten (generalisierte kleinste Quadrate-Schätzung, GLS) und Parameter der iterativ angepaßten sphärischen Semivariogramme

\begin{tabular}{l|rrrr|rrr}
\hline $\begin{array}{c}\text { Monat/ } \\
\text { Jahr }\end{array}$ & \multicolumn{4}{|c|}{ GLS-Modell } & \multicolumn{3}{c}{ Semivariogramm } \\
\cline { 2 - 7 } & $\begin{array}{c}\text { Additive } \\
\text { Konstante }\end{array}$ & $\begin{array}{c}\text { Gelände- } \\
\text { höhe }\end{array}$ & Rechtswert & $\begin{array}{c}\text { Abstand z. } \\
\text { Küste }\end{array}$ & Nugget & Sill & Range \\
\hline Jan & 2277,4 & 1,58 & $-0,00048$ & $-0,00076$ & 1000 & 1510 & 51500 \\
Feb & 1697,5 & 0,96 & $-0,00037$ & & 610 & 710 & 55000 \\
Mär & 2232,1 & 1,41 & $-0,00049$ & $-0,00047$ & 680 & 655 & 48000 \\
Apr & 1157,9 & 1,02 & $-0,00020$ & & 600 & 370 & 125000 \\
Mai & 958,2 & 0,88 & $-0,00012$ & & 580 & 1430 & 124000 \\
Jun & 1723,7 & 1,19 & $-0,00029$ & & 1140 & 1080 & 82000 \\
Jul & 787,6 & 0,79 & & $-0,00117$ & 850 & 1650 & 70000 \\
Aug & 721,0 & 0,90 & & $-0,00082$ & 640 & 1320 & 62000 \\
Sep & 713,4 & 0,98 & & $-0,00170$ & 460 & 2670 & 76000 \\
Okt & 2526,7 & 1,13 & $-0,00053$ & $-0,00168$ & 555 & 1875 & 88000 \\
Nov & 2527,6 & 1,48 & $-0,00051$ & $-0,00173$ & 660 & 2960 & 72000 \\
Dez & 2778,8 & 1,75 & $-0,00060$ & $-0,00082$ & 1100 & 1970 & 59000 \\
\hline Jahr & 19880,8 & 14,27 & $-0,00351$ & $-0,01015$ & 71500 & 102300 & 55000 \\
\hline
\end{tabular}


Die für das Tiefland berechneten Parameter der GLS-Regression und der dazugehörigen Semivariogramme zeigen - anders als für die Berglandmodelle beobachtet - nicht alle eine klare Tendenz im Vergleich zu den Werten der OLSRegression. Lediglich für die lageabhängige Variable Abstand zur Küste wurden in den GLS-Modellen stets Regressionskoeffizienten mit geringerem Betrag ermittelt als für die OLS-Regressionen. Für die Geländehöhe gilt dies nicht von April bis Juni, im Fall des Rechtswertes haben nur die GLS-Regressionskoeffizienten für die Monate Januar bis Mai einen geringeren Betrag als die entsprechenden OLSRegressionskoeffizienten.

Additive Konstante und Nugget zeigen zwei lokale Maxima im Dezember und im Juni. Diese hohen Nuggetwerte zeigen ein starkes Rauschen, eine unerklärte Varianz der Zielvariable bzw. der Residuen in diesen Monaten.

Insgesamt sind alle hier angepaßten Semivariogramme typisch für das sphärische Modell. Beispielhaft seien hier das empirische und das theoretische Semivariogramm zur Beschreibung der räumlichen Autokorrelation der Residuen der iterativ ermittelten GLS-Regressionsmodelle für die durchschnittlichen Niederschlagssumme im Januar abgebildet:

Abbildung 31: Semivariogramme zur Beschreibung der räumlichen
Autokorrelation der Residuen des iterativ ermittelten GLS-
Regressionsmodells für die langjährige durchschnittliche
Niederschlagssumme im Januar für das niedersächsische Tiefland
(193 Stationen); Nugget 1000, Sill 1510, Range 51500

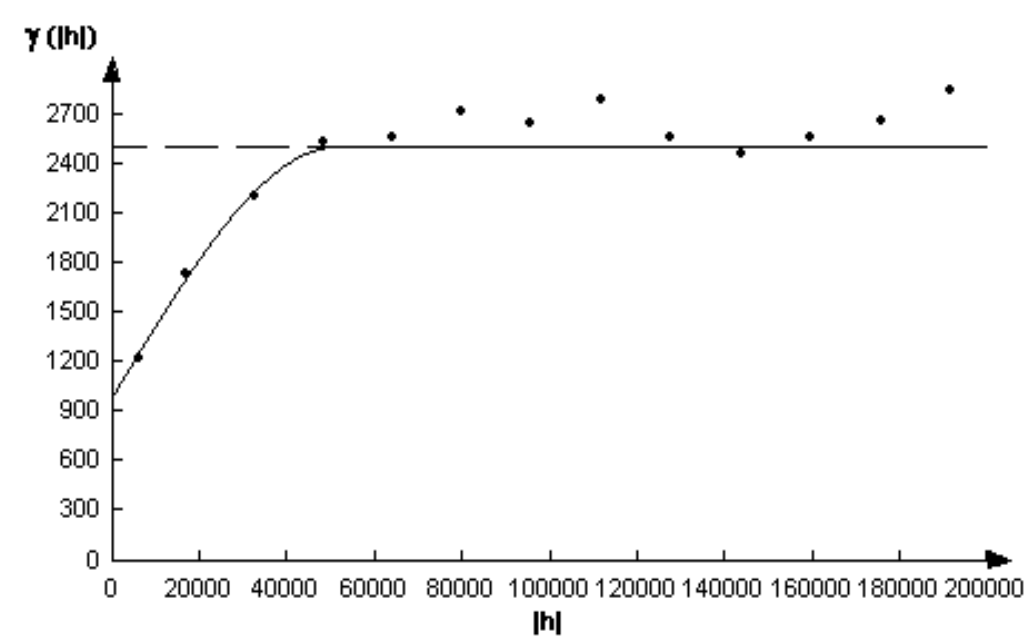

Die gute Modellierbarkeit der Semivariogramme gibt nicht notwendigerweise auch einen Hinweis auf eine starke Verbesserung der Schätzung durch die Modellierung der räumlichen Autokorrelation. Analog zu den Ausführungen zum Bergland wird 128 
die Verbesserung des Schätzung durch eine Kreuzvalidierung im Vergleich mit der Standardabweichung der Residuen der OLS-Regressionen quantifiziert (Tabelle 57).

Tabelle 57: Niederschlagssummen Tiefland, DWD-Datenkollektiv (193 Stationen): Ergebnis der Kreuzvalidierung und Vergleich mit der Modellanpassung nach Regression der Methode der gewöhnlichen kleinsten Quadrate (OLS)

\begin{tabular}{|c|c|c|c|c|}
\hline $\begin{array}{c}\text { Monat/ } \\
\text { Jahr } \\
\text { (1) }\end{array}$ & $\begin{array}{c}\text { STD } \\
\text { residual OLS } \\
{[1 / 10 \mathrm{~mm}]} \\
(2)\end{array}$ & $\begin{array}{c}\text { STD } \\
Z^{*}-\text { residual(GLS) } \\
{[1 / 10 \text { mm }]} \\
(3)\end{array}$ & $\begin{array}{c}\text { Differenz } \\
(2)-(3) \\
{[1 / 10 \mathrm{~mm}]} \\
(4)\end{array}$ & $\begin{array}{c}\text { Relative Differenz } \\
(4) /(2) * 100 \\
{[\%]} \\
(5)\end{array}$ \\
\hline Jan & 49,6 & 41,1 & 8,5 & 17,2 \\
\hline Feb & 35,9 & 30,0 & 5,9 & 16,4 \\
\hline Mär & 36,4 & 31,4 & 4,9 & 13,5 \\
\hline Apr & 30,6 & 26,9 & 3,7 & 12,0 \\
\hline Mai & 43,7 & 28,1 & 15,5 & 35,6 \\
\hline Jun & 46,3 & 36,4 & 9,9 & 21,4 \\
\hline Jul & 49,0 & 36,9 & 12,1 & 24,7 \\
\hline Aug & 44,2 & 35,0 & 9,2 & 20,8 \\
\hline Sep & 54,9 & 31,3 & 23,5 & 42,9 \\
\hline Okt & 48,0 & 30,1 & 17,9 & 37,4 \\
\hline Nov & 59,0 & 39,3 & 19,7 & 33,4 \\
\hline Dez & 54,5 & 42,4 & 12,1 & 22,3 \\
\hline Jahr & 412,3 & 332,8 & 79,5 & 19,3 \\
\hline
\end{tabular}

Die relative Verbesserung der Schätzung durch Berücksichtigung der räumlichen Autokorrelation liegt zwischen $12,0 \%$ und $42,9 \%$. Absolut gesehen ist die Verbesserung mit maximal 2,4 $\mathrm{mm}$ im Monat September eher als bescheiden zu bezeichnen. Im Verhältnis zur langjährigen mittleren Niederschlagssumme in diesem Monat von $61 \mathrm{~mm}$ (vgl. Tabelle 28, S. 80) entspricht dies aber immerhin ca. $4 \%$. Nur in den Monaten März und April wird die 1 \%-Marke knapp unterschritten.

\subsubsection{Relative Luftfeuchte}

Analog zu der in den Kapiteln 5.1.1 und 5.1.2 angewandten Methodik wurde auch das DWD-Datenkollektiv für die durchschnittliche relative Luftfeuchte in ein Bergland- und ein Tiefland-Teilkollektiv geteilt. Hier soll zunächst die Verbesserung der Präzision durch diese Maßnahme quantifiziert werden (s. Tabelle 60). Die Plausibilität dieser Maßnahme kann auf die gleiche Weise begründet werden wie für die beiden anderen Klimaelemente. Erneut ähnelt die Parametrisierung der Gesamtmodelle einer Hybridisierung zwischen Bergland- und Tieflandmodellen (s. Tabelle 61 und Tabelle 62). 
Tabelle 58: Relative Luftfeuchte Niedersachsen, DWD-Datenkollektiv (44 Stationen): Regressionskoeffizienten der monatlichen und jährlichen Modelle

\begin{tabular}{|c|c|c|c|c|c|c|c|c|}
\hline $\begin{array}{c}\text { Monat/ } \\
\text { Jahr }\end{array}$ & $\begin{array}{c}\text { Additive } \\
\text { Konstante }\end{array}$ & Bebauung & Gewässer & \begin{tabular}{|c} 
Lee-Effekt \\
50
\end{tabular} & $\begin{array}{c}\text { Gelände- } \\
\text { höhe }\end{array}$ & expmin & $\begin{array}{l}\text { Hochwert } \\
\text { : }\end{array}$ & $\begin{array}{l}\text { Abstand } \\
\text { z. Küste }\end{array}$ \\
\hline Jan & 31,16 & $-3,356$ & & $-5,55 \mathrm{E}-03$ & & & $9,71 \mathrm{E}-06$ & \\
\hline Feb & 38,43 & $-5,021$ & & $-4,64 \mathrm{E}-03$ & & 0,0482 & 7,92E-06 & \\
\hline Mär & 82,50 & $-6,469$ & 4,687 & $-9,49 \mathrm{E}-03$ & & & & \\
\hline Apr & 76,63 & $-6,587$ & 7,574 & $-8,10 \mathrm{E}-03$ & & 0,0850 & & \\
\hline Mai & 75,46 & $-7,993$ & 7,998 & $-6,62 \mathrm{E}-03$ & & & & \\
\hline Jun & 76,70 & $-6,305$ & 6,294 & $-7,01 \mathrm{E}-03$ & & & & \\
\hline Jul & 78,38 & $-6,692$ & 4,719 & $-7,64 \mathrm{E}-03$ & & & & \\
\hline Aug & 80,62 & $-5,898$ & & & 0,00866 & & & $-2,73 \mathrm{E}-05$ \\
\hline Sep & 83,08 & $-4,457$ & & $-6,89 \mathrm{E}-03$ & & & & \\
\hline Okt & 50,17 & $-4,069$ & & $-3,87 \mathrm{E}-03$ & & & $6,05 \mathrm{E}-06$ & \\
\hline Nov & 62,48 & $-4,105$ & & $-6,36 \mathrm{E}-03$ & & & & \\
\hline Dez & 77,56 & $-4,407$ & & $-4,68 \mathrm{E}-03$ & & & & \\
\hline Jahr & 36,96 & $-5,681$ & & $-6,48 \mathrm{E}-03$ & & & 7,82E-06 & \\
\hline
\end{tabular}

Auffällig ist die häufige Modellbildung unter Einschluß der Variable Lee-Effekt 50. Die Einbeziehung der Variablen der geographischen Lage und vor allem der Variablen der Landnutzung ähnelt derjenigen der Tieflandmodelle (Tabelle 62).

Tabelle 59: Relative Luftfeuchte Niedersachsen, DWD-Datenkollektiv (44 Stationen): Bestimmtheitsmaß $\left(R^{2}\right)$ und Wurzel der mittleren quadrierten Residuen (RMSE) der monatlichen und jährlichen Modelle

\begin{tabular}{l|ll}
\hline $\begin{array}{l}\text { Monat/ } \\
\text { Jahr }\end{array}$ & $\begin{array}{c}\mathbf{R}^{2} \\
{[\%]}\end{array}$ & $\begin{array}{c}\text { RMSE } \\
{[\%]}\end{array}$ \\
\hline Jan & 61,4 & 1,27 \\
Feb & 74,0 & 1,03 \\
Mär & 80,4 & 1,29 \\
Apr & 83,3 & 1,33 \\
Mai & 71,7 & 1,74 \\
Jun & 67,4 & 1,67 \\
Jul & 69,8 & 1,56 \\
Aug & 73,7 & 1,36 \\
Sep & 62,3 & 1,29 \\
Okt & 55,8 & 1,15 \\
\cline { 2 - 3 } Nov & 62,2 & 1,28 \\
Dez & 60,0 & 1,21 \\
\hline Jahr & 73,4 & 1,16 \\
\hline
\end{tabular}

Die Modelle für das Niedersachsendatenkollektiv können auch einen erheblichen Anteil der Varianz der Zielvariablen aufklären (s. Tabelle 59). Um wieviel genauer 
die Modelle der Teilkollektive sind, wurde anhand der Summen der quadrierten Residuen in der folgenden Tabelle 60 verglichen.

Tabelle 60: Vergleich von Summen der quadrierten Residuen (SQR) für gemeinsame Modellierung (MOD: NDS) und für getrennte Modelle der relativen Luftfeuchte (MOD: TIEF, bzw. MOD: BERG); in Klammern die Anzahl der Regressoren

\begin{tabular}{|c|c|c|c|c|c|c|c|c|c|}
\hline \multirow[b]{2}{*}{$\begin{array}{c}\text { Stationen } \\
\mathbf{N}\end{array}$} & \multicolumn{3}{|c|}{ MOD: NDS } & \multicolumn{2}{|c|}{ Vorteil spez. Modell } & \multicolumn{4}{|c|}{ MOD: TIEF MOD: BERG } \\
\hline & $\begin{array}{c}\text { Gesamt } \\
44\end{array}$ & $\begin{array}{c}\text { Tiefland } \\
29\end{array}$ & $\begin{array}{c}\text { Bergland } \\
15\end{array}$ & $\begin{array}{c}\text { Tiefland } \\
29\end{array}$ & $\begin{array}{c}\text { Bergland } \\
15\end{array}$ & & & Ber & \\
\hline Jan & $64,2(3)$ & 35,3 & 28,8 & $-1,3$ & $-0,2$ & 36,6 & (1) & 29,0 & $(1)$ \\
\hline Feb & 41,1 (4) & 29,5 & 11,6 & 2,5 & $-0,7$ & 27,0 & (3) & 12,3 & $(2)$ \\
\hline Mär & 66,8 (3) & 38,3 & 28,5 & 18,1 & 9,9 & 20,2 & (4) & 18,7 & $(2)$ \\
\hline Apr & $69,3(4)$ & 43,9 & 25,5 & 18,8 & 10,6 & 25,1 & (4) & 14,9 & $(3)$ \\
\hline Mai & 120,9 (3) & 74,9 & 46,0 & 17,4 & 33,5 & 57,5 & (3) & 12,5 & $(3)$ \\
\hline Jun & 112,2 (3) & 51,0 & 61,2 & 16,4 & 26,0 & 34,6 & (3) & 35,3 & $(2)$ \\
\hline Jul & $97,7 \quad(3)$ & 49,7 & 48,0 & 25,9 & $-8,9$ & 23,8 & (3) & 56,9 & $(1)$ \\
\hline Aug & 73,9 (3) & 33,2 & 40,7 & 11,3 & 21,6 & 21,9 & (3) & 19,2 & (3) \\
\hline Sep & 67,9 (2) & 36,3 & 31,6 & $-2,4$ & $-2,8$ & 38,7 & (1) & 34,4 & (1) \\
\hline Okt & 52,7 (3) & 29,4 & 23,3 & 0,7 & $-0,9$ & 28,7 & (1) & 24,3 & $(1)$ \\
\hline Nov & 65,4 (3) & 38,8 & 26,6 & 1,0 & $-12,6$ & 37,7 & (1) & 39,2 & $(1)$ \\
\hline Dez & $58,7 \quad(3)$ & 34,0 & 24,7 & $-1,5$ & $-4,2$ & 35,5 & (1) & 28,9 & (1) \\
\hline Jahr & $54,2(3)$ & 30,9 & 23,3 & 6,3 & 13,8 & 24,6 & (3) & 9,5 & (3) \\
\hline
\end{tabular}

Diejenigen negativen Differenzen, die eine höhere Präzision desjenigen Modells anzeigen, das mit dem gemeinsamen Datenkollektiv ermittelt wurde, sind in Tabelle 60 grau unterlegt. Sie treten nur auf, wo das Modell des gemeinsamen Datenkollektivs mehr Regressoren verwendet als das unterlegene Modell für das Teilkollektiv. Alle anderen Werte belegen die höhere Präzision von Modellen der Teilkollektive. Bis auf den Vergleich des Tieflandmodells für März verwenden alle Teilkollektivmodelle gleich viele oder weniger Regressoren als das jeweilige Modell des Gesamtkollektivs. Vor allem für das Tiefland zeigt sich eine Erhöhung der Präzision.

\subsubsection{Relative Luftfeuchte im Bergland}

Die Regionalisierung der relativen Luftfeuchte im Bergland stützt sich auf Daten von 15 Stationen des DWD (s. Kap. 4.1.4.2). Analog zu der Vorgehensweise bei der Regionalisierung der Lufttemperatur wurde auch hier eine schrittweise lineare Regression durchgeführt und auf eine anschließende Anwendung geostatistischer Verfahren wegen des zu niedrigen Stichprobenumfanges verzichtet. 
Da ein starker Zusammenhang der relativen Luftfeuchte $\mathrm{zu}$ Wasserangebot und Lufttemperatur besteht, ist nach den Ergebnissen der Kapitel 5.1.1.1 und 5.1.2.1 auch für die Regionalisierung der relativen Luftfeuchte im Bergland ein hohes Erklärungspotential der Geländehöhe zu erwarten. Wie Tabelle 61 zeigt, wird diese Variable für einige Monate noch durch die Variable Lee-Effekt 50 übertroffen (s. Kap. 3.3.3).

Tabelle 61: Relative Luftfeuchte Bergland, DWD-Datenkollektiv (15 Stationen): Regressionskoeffizienten, Bestimmtheitsmaß $\left(\mathbf{R}^{2}\right)$ und Wurzel der mittleren quadrierten Residuen (RMSE) der monatlichen und jährlichen Modelle

\begin{tabular}{l|cc|c:cc:c:c}
\hline $\begin{array}{c}\text { Monat/ } \\
\text { Jahr }\end{array}$ & $\begin{array}{c}\mathbf{R}^{2} \\
{[\%]}\end{array}$ & $\begin{array}{c}\text { RMSE } \\
{[\%]}\end{array}$ & $\begin{array}{c}\text { Additive } \\
\text { Konstante }\end{array}$ & $\begin{array}{c}\text { Gelände- } \\
\text { höhe }\end{array}$ & $\begin{array}{c}\text { Lee Effekt } \\
\mathbf{5 0}\end{array}$ & Bebauung & Rechtswert \\
\hline Jan & 66,2 & 1,49 & 85,98 & & $-6,88 \mathrm{E}-03$ & & \\
Feb & 84,8 & 1,01 & 85,08 & & $-5,05 \mathrm{E}-03$ & $-6,106$ & \\
Mär & 91,4 & 1,25 & 78,84 & 0,00960 & & $-8,333$ & \\
Apr & 92,8 & 1,16 & 193,38 & 0,01056 & & & $-3,31 \mathrm{E}-05$ \\
Mai & 92,1 & 1,07 & 204,54 & 0,00877 & & $-8,993$ & $-3,67 \mathrm{E}-05$ \\
Jun & 77,0 & 1,71 & 214,27 & 0,01120 & & $-9,426$ & $-3,98 \mathrm{E}-05$ \\
Jul & 84,6 & 1,54 & 250,63 & 0,00904 & & $-8,961$ & $-4,89 \mathrm{E}-05$ \\
Aug & 85,9 & 1,32 & 244,88 & 0,00859 & & $-7,257$ & $-4,73 \mathrm{E}-05$ \\
Sep & 71,7 & 1,63 & 82,02 & & $-8,53 \mathrm{E}-03$ & & \\
Okt & 57,9 & 1,37 & 83,74 & & $-5,27 \mathrm{E}-03$ & & \\
Nov & 62,9 & 1,74 & 86,09 & & $-7,44 \mathrm{E}-03$ & & \\
Dez & 62,7 & 1,49 & 87,08 & & $-6,36 \mathrm{E}-03$ & & \\
\hline Jahr & 91,2 & 0,93 & 167,93 & 0,00748 & & $-6,792$ & $-2,47 \mathrm{E}-05$ \\
\hline
\end{tabular}

Die Bestimmtheitsmaße aller Modelle sind zufriedenstellend hoch und passen so zu den niedrigen Werten des RMSE. Allerdings ist die Standardabweichung der Meßwerte meist nur ca. doppelt so groß (vgl. Tabelle 31, S. 83).

Die monatlichen Modelle werden grundsätzlich entweder ausschließlich mit dem Lee-Effekt 50 (Herbst und Winter), im Februar in Kombination mit Bebauung oder durch Kombinationen von Geländehöhe, Bebauung und Rechtswert gebildet (Frühling und Sommer). Die höhenabhängige Variable zeigt jeweils mit Abstand das größte Erklärungspotential für die Streuung der Zielvariablen. Das Bestimmtheitsmaß für nur durch die Geländehöhe bzw. den Lee-Effekt 50 gebildete Modelle liegt stets zwischen $57 \%$ und $87 \%$. 


\subsubsection{Relative Luftfeuchte im Tiefland}

Die Modelle zur Regionalisierung der relativen Luftfeuchte im Tiefland (Tabelle 62) stützen sich auf die gleichen 29 Stationen wie die Temperaturmodelle. Die monatlichen Modelle zur Regionalisierung der relativen Luftfeuchte im Tiefland zeigen eine hohe Ähnlichkeit mit den Temperaturmodellen (s. Tabelle 46, S. 112). Eine starke Abweichung ist jedoch das völlige Fehlen des Rechtswertes in den Modellen der relativen Luftfeuchte. Der Anteil von Bebauung an der Landnutzung ist die konsistente Komponente der Feuchtemodelle. In den Herbst- und Wintermonaten (September bis Januar) konnte ein bedeutender Zusammenhang mit der relativen Luftfeuchte ausschließlich zu dieser räumlichen Variablen gefunden werden. Durch diese Modelle ließ sich die Varianz der Zielvariablen relative Luftfeuchte nur zu unter $35 \%$ erklären. Die Bestimmtheitsmaße der restlichen Modelle, in denen auch starke Korrelationen mit anderen räumlichen Variablen genutzt werden konnten, sind deutlich höher.

Welchen Anteil die beteiligten Variablen am Bestimmtheitsmaß der jeweiligen Modelle haben, wird zunächst nicht aus den Modellen ersichtlich. Daher wurden wie in Kap. 5.1.1.2 die Beiträge zum Bestimmtheitsmaß aus der schrittweisen Regression in Form eines Histogramms dargestellt (s. Abbildung 32). Hieraus geht die Bedeutung der einzelnen Variablen für die Regionalisierung der relativen Luftfeuchte hervor. Es wird deutlich, daß vor allem die Variablen der geographischen Lage (Hochwert und Abstand z. Küste) einen großen Anteil der Zielvariablenvarianz aufklären können, wo sie denn überhaupt einen bedeutenden Zusammenhang mit der relativen Luftfeuchte zeigen. Die Geländehöhe oder die Exponiertheit zum Umgebungsminimum sind von untergeordneter Rolle. Auch der Anteil der Gewässer an der Landnutzung ist nur an vier Modellen (März bis Juni) beteiligt und erklärt dort nur stets unter $10 \%$ der Zielvariablenvarianz. 
Tabelle 62: Relative Luftfeuchte Tiefland, DWD-Datenkollektiv (29 Stationen): Regressionskoeffizienten, Bestimmtheitsmaß $\left(\mathbf{R}^{2}\right)$ und Wurzel der mittleren quadrierten Residuen (RMSE) der monatlichen und jährlichen Modelle

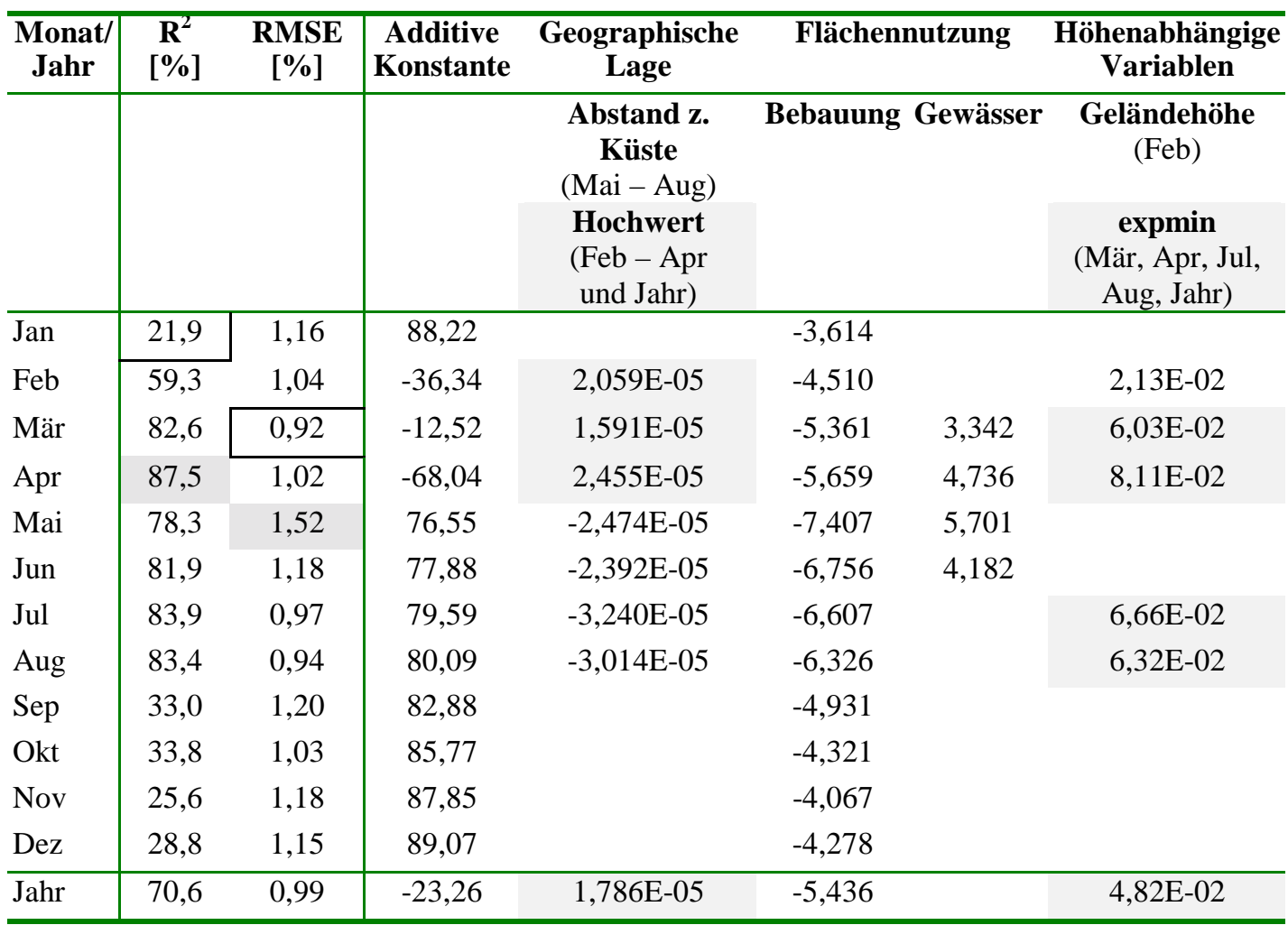

Abbildung 32: Relative Luftfeuchte, DWD-Tieflanddatenkollektiv (29 Stationen): Beiträge zum Bestimmtheitsmaß der räumlichen Variablen

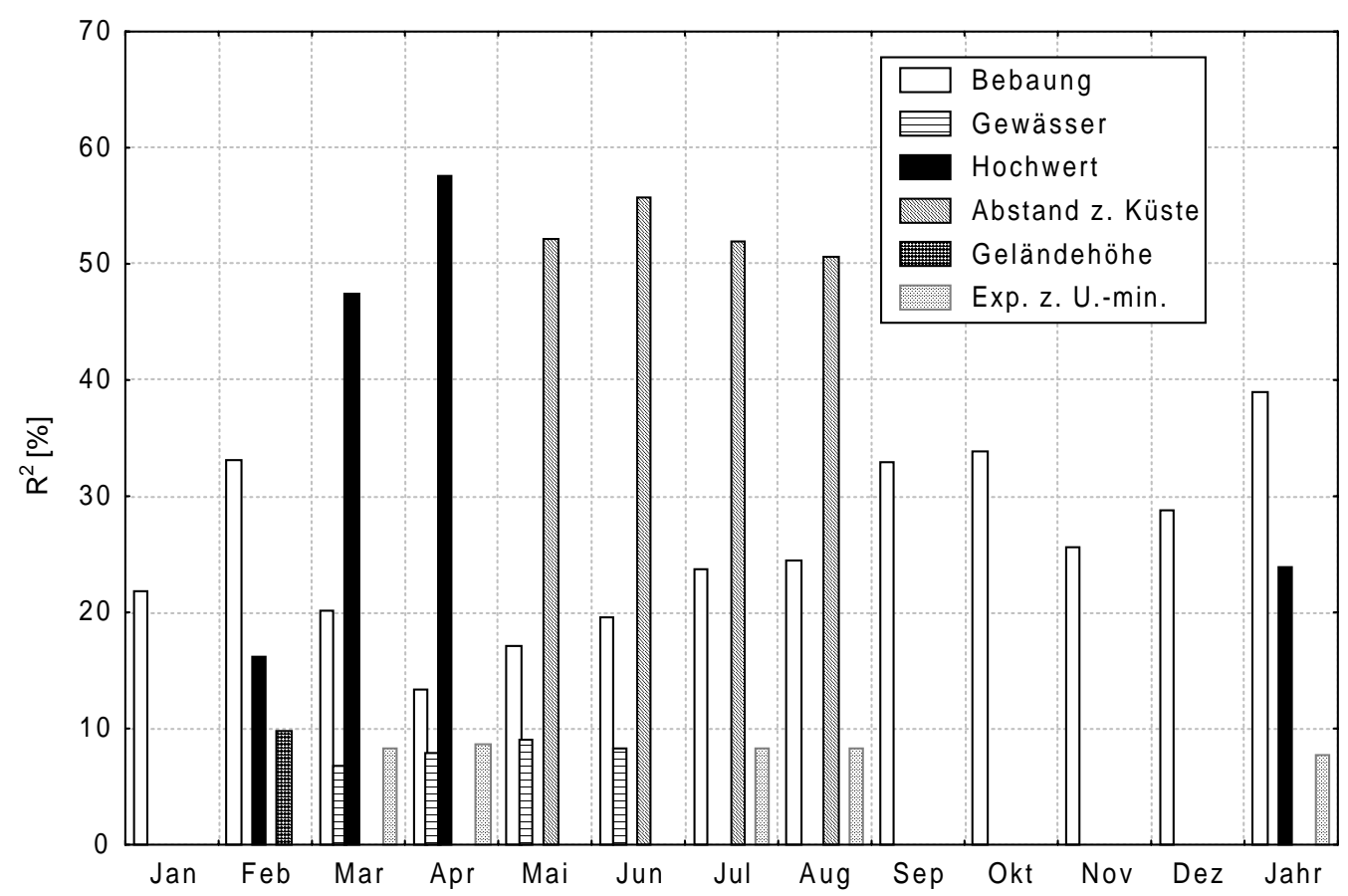




\subsection{Depositionen}

In Kap. 4.2 und speziell in Kap. 4.2.3 wurde bereits beschrieben, daß in gesamt Niedersachsen nur Daten (Mittelwerte von 1986 bis 1990) von maximal 10 Waldstationen verwendet werden konnten und im Rahmen der vorliegenden Arbeit gerade Aussagen über Waldstandorte gemacht werden sollten. Es konnte daher nur eine gemeinsame Datenanalyse für Berg- und Tiefland erfolgen. Die Verwendung der individuell regionalisierten Variablen Jährliche Niederschlagssumme (s. Kap. 5.1.2) in der schrittweisen linearen Regression sollte eventuelle Nachteile durch die gemeinsame Regionalisierung vermeiden. Unter der Annahme, daß außer der Höhe des Niederschlages keine weiteren Einflußgrößen auf die Depositionsmenge einen für Berg- und Tiefland unterschiedlichen Einfluß zeigen, ist eine Unterteilung des Zielgebietes zur Depositionsregionalisierung damit nicht nötig.

Die Gesamtdepositionen für Waldbestände ausgehend von durchschnittlichen Freilanddepositionen berechneten LENZ et al. (1996) über Anreicherungsfaktoren bzw. über einen wissensbasierten Algorithmus. Diese Art der Berechnung von Gesamtdepositionen ,setzt die Kenntnis der durchschnittlichen Freilanddepositionen (elementspezifisch) voraus“ (LENZ et al., 1996). Die Regionalisierungsmodelle für die Freilanddepositionen sollen daher zunächst dargestellt werden. Sie sind im Sinne der gewählten Vorgehensweise (Kap. 4.2.4) Voraussetzung für die nachfolgend im Kapitel 5.2.2 dargestellten Modelle zur Integration der Walddepositionen.

Die Untersuchung der Regressionsresiduen der Freilandmodelle zeigte starke Abweichungen von der Voraussetzung der Stationarität und keine bedeutende räumliche Autokorrelation, weshalb auf eine Diskussion geostatistischer Untersuchungen in diesem Kapitel verzichtet wird.

\subsubsection{Ausschließliche Betrachtung der Freilanddepositionen}

Die durch schrittweise lineare Regression gewonnenen Modelle sind in Tabelle 63, die zugehörigen Bestimmtheitsmaße und mittleren Fehler (RMSE) in Tabelle 64 aufgeführt. Die Einteilung der räumlichen Variablen in die aus Kap. 3 bekannten Gruppen dient lediglich einer besseren Übersicht. 
Tabelle 63: Regressionskoeffizienten der Modelle für die Deposition der untersuchten fünf Elemente und den pH-Wert im Freilandniederschlag (Bulk-Depositionen); gesamt Niedersachsen

\begin{tabular}{|c|c|c|c|c|c|c|c|c|c|}
\hline & \multirow[b]{2}{*}{$\begin{array}{l}\text { Add. } \\
\text { Konst. }\end{array}$} & \multicolumn{3}{|c|}{ höhenabhängige Variablen } & \multicolumn{3}{|c|}{ Landnutzung } & \multicolumn{2}{|c|}{ geogr. Lage } \\
\hline & & $\begin{array}{c}\text { Gelände- } \\
\text { höhe }\end{array}$ & Nied.Jahr & expmin & Bebauung & Gewässer & Wald & Rechtsw & $\begin{array}{l}\text { Abstand } \\
\text { z. Küste }\end{array}$ \\
\hline SO4-S & 13,94 & 0,01420 & & & 8,60 & & $-5,73$ & & \\
\hline NO3-N & 5,22 & 0,00568 & & & & & & & \\
\hline NH4-N & 64,04 & & 0,00056 & & & $-6,01$ & & $-1,7 \mathrm{E}-05$ & \\
\hline Cl & 27,89 & & & & & 161,43 & & & $-9,70 \mathrm{E}-05$ \\
\hline $\mathbf{H}$ & $-1,88$ & 0,00040 & & & & & & $5,8 \mathrm{E}-07$ & \\
\hline pH & 13,69 & & & $-0,0081$ & & & & $-2,5 \mathrm{E}-06$ & \\
\hline
\end{tabular}

Der Jahresniederschlag ist hier als Summe der modellierten monatlichen Niederschlagssummen definiert worden, was eine höhere und vor allem feiner strukturierte Variabilität verspricht als die Verwendung der Regionalisierungsfunktion für die durchschnittliche jährliche Niederschlagssumme.

Die Variable Geländehöhe kann einen bedeutenden Teil der Einträge an SulfatSchwefel, Nitrat-Stickstoff und Protonen erklären. Der Jahresniederschlag - nicht ausschließlich „höhenabhängig“ - übertrifft bezüglich des Erklärungspotentials für die Varianz der Ammonium-Stickstoffeinträge die Geländehöhe knapp. Beide Variablen zeigen positive Korrelationen mit den Ammonium-Stickstoffdepositionen. Die dritte höhenabhängige Variable, die Exponiertheit zum Umgebungsminimum (expmin), findet bei der Regionalisierung des $\mathrm{pH}-$ Wertes Verwendung. Der negative Regressionskoeffizient deutet darauf hin, daß die Niederschläge an exponierten Punkten des Geländes saurer sind als diejenigen an weniger exponierten Stellen.

Zur Regionalisierung der Deposition von Sulfat-Schwefel wurden sowohl Bebauung als auch Wald verwendet. Diese sind definitionsgemäß voneinander abhängig. Der Korrelationskoeffizient nach Pearson beider Variablen im SchwefelFreilandkollektiv hat den Wert $-0,49$ und ist hoch signifikant. Beide Variablen verbleiben trotz dieser Abhängigkeit in dem Modell, weil sie jeweils eine eindeutige und plausible Beziehung mit der Zielvariablen zeigen. Während für Bebauung ein positiver Regressionskoeffizient ermittelt wurde - je mehr Siedlungen in der Umgebung desto höher die Sulfat-Schwefeleinträge -, ist ein größerer Waldanteil in der Stationsumgebung mit geringeren Depositionen von Sulfat-Schwefel verbunden. Eine Reduktion des Modells um eine der beiden Variablen erscheint daher nicht sinnvoll. Der Eintrag an Ammonium-Stickstoff sinkt, der Chloreintrag steigt mit zunehmendem Gewässeranteil in der Stationsumgebung. Letzteres ist vor allem auf 
die drei Freilandstationen mit den höchsten Chlor-Einträgen zurückzuführen: „Norderney Stadt“ (57,5\% Gewässeranteil), „Norderney-Flughafen“ (46,2\%) und „Emden-Knock“ (66,5\%). Für die Station mit dem vierthöchsten Gewässeranteil, „Nordenham/City“, wurde nur noch ein Gewässeranteil von $10 \%$ ermittelt.

Einen ähnlichen Effekt wie Gewässer zeigt die lageabhängige Variable Abstand zur Küste für den Chloreintrag. Das Modell weist so für eine Entfernung von $100 \mathrm{~km}$ zur Küste einen um 9,7 kg (ha•a) ${ }^{-1}$ geringeren Chloreintrag aus. Die zweite lageabhängige Variable, der Rechtswert, erklärt einen Teil der Zielvariablenvarianzen für Ammonium-Stickstoff und den pH-Wert. Im Osten werden bei geringeren Einträgen an Ammonium-Stickstoff niedrigere $\mathrm{pH}-$ Werte und höhere Protoneneinträge modelliert.

Tabelle 64: Bestimmtheitsmaß $\left(\mathbf{R}^{2}\right)$ und Wurzel der mittleren quadrierten Residuen (RMSE), Mittelwert der Meßwerte und der zum Mittelwert relative Fehler $($ C.V.=RMSE*100/Mittelw. d. Meßwerte) der Modelle für die Deposition der untersuchten fünf Elemente und den pH-Wert im Freilandniederschlag

\begin{tabular}{|l|cccc|}
\hline & $\begin{array}{c}\mathbf{R}^{\mathbf{2}} \\
{[\%]}\end{array}$ & $\begin{array}{c}\text { RMSE } \\
{\left[\mathbf{k g} /(\mathbf{h a ~ a})^{-\mathbf{1}}\right] \mathbf{~ b z w . ~}[\mathbf{p H}]}\end{array}$ & $\begin{array}{c}\text { C.V. } \\
{[\%]}\end{array}$ & $\begin{array}{c}\text { Mittelw. d. Meßwerte } \\
{\left[\mathbf{k g} /(\mathbf{h a} \mathbf{a})^{-\mathbf{1}}\right] \mathbf{~ b z w . ~}[\mathbf{p H}]}\end{array}$ \\
\hline SO4-S & 68,4 & 1,9 & 13,3 & 14,3 \\
NO3-N & 60,7 & 0,7 & 11,7 & 6,0 \\
NH4-N & 47,3 & 1,6 & 18,2 & 8,8 \\
Cl & 92,0 & 7,3 & 32,7 & 22,3 \\
H & 64,9 & 0,095 & 48,5 & 0,196 \\
\hdashline $\mathbf{p H}$ & 61,8 & 0,2 & 4,3 & 4,7 \\
\hline
\end{tabular}

Die Bestimmtheitsmaße der Regressionsmodelle für den Eintrag von SulfatSchwefel, Nitrat-Stickstoff, Protonen und für die pH-Werte der gesammelten Proben liegen zwischen 60 und 70 Prozent. Das Modell für den Eintrag von AmmoniumStickstoff erreicht nur $47 \%$, das für den Chloreintrag allerdings $92 \%$.

Die Werte des RMSE liegen bis auf denjenigen für den Chloreintrag allesamt unter $2 \mathrm{~kg}(\mathrm{ha} \bullet)^{-1}$, wobei diese absoluten Werte wegen unterschiedlicher Molekülmassen der Zielvariablen nicht zum Vergleich der Modelle herangezogen werden können. Der RMSE für das pH-Modell liegt mit 0,2 bei gut $10 \%$ des Bereichs der durchschnittlichen Meßwerte von 1,9 (pH-Werte von minimal 3,6 bis maximal 5,5). Diese Angabe erscheint bei einem Konzentrationswert wie dem $\mathrm{pH}$ sinnvoller als der Vergleich von RMSE und Mittelwert der abhängigen Variablen. Der aus eben diesem Verhältnis berechnete Variationskoeffizient C.V. (Tabelle 64) zeigt vor allem für den Protonen- und den Chloreintrag sehr schlechte Werte. Der Gegensatz 
zwischen sehr gutem Bestimmtheitsmaß und hohen RMSE-Werten für das Chlormodell soll in der Diskussion (Kap. 6.2.1) näher analysiert werden.

Neben der bisher dargestellten Modellierung für gesamt Niedersachsen wurden die Freilanddepositionen auch getrennt für Berg- und Tiefland regionalisiert. Die so entstandenen Modelle können zur Anwendung bei ausschließlich auf das Freiland bezogenen Fragestellungen oder als Grundlage für wissensbasierte Modelle zur Quantifizierung der Walddepositionen dienen (z. B. LENZ et al., 1996). Auf eine nähere Erläuterung und Diskussion dieser Ergebnisse wird aber im Rahmen dieser Arbeit verzichtet.

Tabelle 65: Regressionskoeffizienten der Modelle für die Deposition der untersuchten fünf Elemente und den pH-Wert im Freilandniederschlag (Bulk-Depositionen); getrennt für niedersächsisches Berg- und Tiefland

\begin{tabular}{|c|c|c|c|c|c|c|c|c|c|}
\hline & \multirow[b]{2}{*}{$\begin{array}{l}\text { Add. } \\
\text { Konst }\end{array}$} & \multicolumn{2}{|c|}{$\begin{array}{c}\text { höhenabhängige } \\
\text { Variablen }\end{array}$} & \multicolumn{2}{|c|}{ Landnutzung } & \multicolumn{3}{|c|}{ geogr. Lage } \\
\hline & & & $\begin{array}{l}\text { Gelände- } \\
\text { höhe }\end{array}$ & Luv & $\begin{array}{c}\text { Bebau- } \\
\text { ung }\end{array}$ & Gewässer & Rechtswert & Hochwert & $\begin{array}{l}\text { Abstand } z . \\
\text { Küste }\end{array}$ \\
\hline \multirow{2}{*}{ SO4-S } & Bergl. & 14,45 & & & 12,077 & & & & \\
\hline & Tiefl. & 11,48 & & & 12.093 & 8.279 & & & \\
\hline \multirow{2}{*}{ NO3-N } & Bergl. & 5,12 & 0,00591 & & & & & & \\
\hline & Tiefl. & 5,18 & $0.0068 \mathrm{C}$ & & & & & & \\
\hline \multirow{2}{*}{ NH4-N } & Bergl. & 103,97 & 0,00865 & & & & $-2,78 \mathrm{E}-05$ & & \\
\hline & Tiefl. & 74,73 & & 0.03986 & & -5.205 & $-1,92 \mathrm{E}-05$ & & \\
\hline \multirow{2}{*}{ Cl } & Bergl. & 204,37 & & & & & $-5,41 \mathrm{E}-05$ & & \\
\hline & Tiefl. & 30,45 & & & & 157,407 & & & $-1,39 E-04$ \\
\hline \multirow{2}{*}{$\mathbf{H}$} & Bergl. & 0,11 & 0,00055 & & & & & & \\
\hline & Tiefl. & $-2,58$ & & & & & $7,85 \mathrm{E}-07$ & & \\
\hline \multirow{2}{*}{ pH } & Bergl. & $-36,54$ & & & & 47,757 & $1,56 \mathrm{E}-06$ & $6,16 \mathrm{E}-06$ & \\
\hline & Tiefl. & 14,94 & & & & & $-2,92 \mathrm{E}-06$ & & \\
\hline
\end{tabular}

Bezogen auf die Präzision der Modelle läßt sich keine klare Tendenz herausarbeiten (s. Tabelle 66). Sehr niedrig ist mit $12,2 \%$ das Bestimmtheitsmaß des Nitratmodells für das niedersächsische Tiefland. Den Ergebnissen für ganz Niedersachsen entsprechend fällt erneut das sehr hohe Bestimmtheitsmaß für das ChlorTieflandmodell in Verbindung mit dem sehr schlechten Variationskoeffizienten C.V. auf. Ähnlich hoch ist der C.V. nur bei den Protonenmodellen, wobei diese auch schlechtere Bestimmtheitsmaße zeigen. 
Tabelle 66: Bestimmtheitsmaß $\left(\mathbf{R}^{2}\right)$ und Wurzel der mittleren quadrierten Residuen (RMSE), Mittelwert der Meßwerte und der zum Mittelwert relative Fehler (C.V.) der Modelle für die Deposition der untersuchten fünf Elemente und den pH-Wert im Freilandniederschlag; getrennt für niedersächsisches Berg- und Tiefland

\begin{tabular}{|c|c|c|c|c|c|}
\hline & & $\begin{array}{l}\mathbf{R}^{2} \\
{[\%]}\end{array}$ & $\begin{array}{c}\text { RMSE } \\
{[\mathrm{kg} /(\mathrm{ha} \text { a) }] \text { bzw. }[\mathrm{pH}]}\end{array}$ & $\begin{array}{l}\text { C.V. } \\
{[\%]}\end{array}$ & $\begin{array}{l}\text { Mittelw. d. Meßwerte } \\
{[\mathrm{kg} /(\text { ha a)] bzw. }[\mathrm{pH}]}\end{array}$ \\
\hline \multirow{2}{*}{ SO4-S } & Bergl. & 49,0 & 2,1 & 13,3 & 15,8 \\
\hline & Tief. & 65,5 & 2,0 & 14,5 & 13.6 \\
\hline \multirow{2}{*}{ NO3-N } & Bergl. & 54,1 & 0,8 & 12,1 & 7,0 \\
\hline & Tiefi. & 12,2 & 0,7 & 11,9 & 5,5 \\
\hline \multirow{2}{*}{ NH4-N } & Bergl. & 66,4 & 1,3 & 14,4 & 8,8 \\
\hline & Tiefl. & 66,3 & 1,3 & 15.0 & 8,8 \\
\hline \multirow{2}{*}{ Cl } & Bergl. & 81,4 & 1,7 & 13,8 & 12,5 \\
\hline & Tiefl. & 92,8 & 8,1 & 30,1 & 27.0 \\
\hline \multirow{2}{*}{$\mathbf{H}$} & Bergl. & 47,7 & 0,10 & 32,9 & 0,30 \\
\hline & Tiefl. & 59,7 & 0.05 & 36.7 & 0,15 \\
\hline \multirow{2}{*}{ pH } & Bergl. & 83,7 & 0,11 & 2,4 & 4,54 \\
\hline & Tief. & 62,5 & 0,19 & 3,9 & 4,82 \\
\hline
\end{tabular}

\subsubsection{Integration der Bestandesdepositionen}

Wie in Kap. 4.2.4 dargestellt, wurden die Bestandesdepositionen als Produkt der regionalisierten Freilanddepositionen (Kap. 5.2.1) und des Anreicherungsfaktors definiert. Die aus Bestandesmeßdaten und regionalisierten Freilanddepositionen ermittelten Anreicherungsfaktoren wurden nach der in Kap. 2.5 beschriebenen Methodik als Zielvariablen regionalisiert. Wegen des sehr geringen Stichprobenumfangs sind alle Aussagen trotz teils sehr hoher Bestimmtheitsmaße mit Vorsicht zu interpretieren.

Tabelle 67: Regressionskoeffizienten der Modelle für die Anreicherungsfaktoren der untersuchten fünf Elemente und des pHWertes von Freilandniederschlag zu Bestandesniederschlag

\begin{tabular}{|c|c|c|c|c|c|c|c|}
\hline & Add. Konst & Nied.Jahr & Luv & Buche & Fichte & Acker & $\begin{array}{c}\text { Abstand z. } \\
\text { Küste } \\
\end{array}$ \\
\hline SO4-S & $-3,1$ & 0,000791 & & $-2,500$ & & & \\
\hline NO3-N & 0,8 & & 0,0085 & & 1,639 & & \\
\hline NH4-N & 1,3 & & & & 1,933 & 1,855 & \\
\hline Cl & $-27,4$ & 0,004006 & & & & & $-3,039 \mathrm{e}-005$ \\
\hline H & $-5,6$ & 0,000889 & & & & & \\
\hline $\mathbf{p H}$ & 0,9 & & & & $-0,076$ & 0,244 & \\
\hline
\end{tabular}

Die Regressionskoeffizienten der gefunden Modelle für die Anreicherungsfaktoren zeigen plausible Vorzeichen. Überdurchschnittlich hohe Niederschläge führen ebenso zu höheren Depositionen wie die Lage in Luvgebieten oder die Bestockung mit Fichte. Die Bestockung mit Buche führt im Vergleich zu anderen Baumarten 
$(6 \times$ Fichte, $1 \times$ Kiefer, $1 \times$ Eiche) zu unterdurchschnittlichen Einträgen an Sulfat. Die Einträge an Ammonium-Stickstoff sind bei einem hohen Wert für Acker (Anteil landwirtschaftlich genutzter Flächen in einem Umkreis von $2 \mathrm{~km}$ ) höher, der pHWert steigt mit dieser Variable. Mit zunehmendem Abstand zur Küste wird die Menge deponierten Chlors geringer.

Die Höhe der Regressionskoeffizienten führt bei einigen Modellen (für SulfatSchwefel-, Chlor- und Protoneneinträge) dazu, daß für das Wertespektrum der räumlichen Variablen in Niedersachsen unplausibel negative Anreicherungsfaktoren berechnet werden. Während noch Anreicherungsfaktoren von 0 bis 1 einer Reduktion des Eintrages von Freiland $\mathrm{zu}$ Bestandesniederschlag entsprechen und somit grundsätzlich mit Kronenraum-Regen-Interaktionen erklärt werden können, gibt es für negative Anreicherungsfaktoren keine plausible Erklärung. Negative Anreicherungsfaktoren entsprechen einem Austrag mit dem Bestandesniederschlag, was schlicht unmöglich ist. Selbst wenn das engere Wertespektrum der Waldmeßstationen vorausgesetzt wird, sind negative Modellwerte möglich, auch wenn für die 10 (bzw. 9) Waldstationen durch die vorliegende Kombination der Variablenwerte keine negativen Anreicherungsfaktoren berechnet werden. Dieses Problem wird in Kap. 6.2.2 näher besprochen.

Die Bestimmtheitsmaße für die Modelle der Anreicherungsfaktoren für SulfatSchwefel, Ammonium-Stickstoff, Nitrat-Stickstoff und Chlor liegen zwischen $85 \%$ und $95 \%$ (s. Tabelle 68).

Tabelle 68: Bestimmtheitsmaß $\left(\mathbf{R}^{2}\right)$ und Wurzel der mittleren quadrierten Residuen (RMSE), Mittelwert der Anreicherungsfaktoren und der zum Mittelwert relative Fehler (C.V.) der Modelle für die räumliche Verteilung der Anreicherungsfaktoren von Freilanddepositionen zu Bestandesniederschlägen der untersuchten fünf Elemente und des pHWertes

\begin{tabular}{|c|c|c|c|c|}
\hline & $\begin{array}{c}\mathrm{R}^{2} \\
{[\%]}\end{array}$ & $\begin{array}{c}\text { RMSE } \\
{[\mathrm{kg} /(\mathbf{h a} \text { a })] \text { bzw. }[\mathrm{pH}]}\end{array}$ & $\begin{array}{l}\text { C.V. } \\
{[\%]}\end{array}$ & $\begin{array}{c}\text { Mittelw. d. A.-faktoren } \\
{[\mathrm{kg} /(\mathrm{ha} \text { a })] \text { bzw. }[\mathrm{pH}]}\end{array}$ \\
\hline SO4-S & 91,0 & 0,65 & 17,4 & 3,77 \\
\hline NO3-N & 87,4 & 0,46 & 20,4 & 2,25 \\
\hline NH4-N & 90,5 & 0,46 & 16,1 & 2,87 \\
\hline Cl & 94,8 & 1,64 & 28,1 & 5,85 \\
\hline $\mathbf{H}$ & 54,5 & 1,69 & 62,6 & 2,70 \\
\hline $\mathbf{p H}$ & 76,1 & 0,04 & 4,5 & 0,91 \\
\hline
\end{tabular}

Das Modell für den Protonenanreicherungsfaktor kann nur 54,5\% der Zielvariablenvarianz erklären, das für den pH-Wert-Anreicherungsfaktor immerhin $76,1 \%$. Eine hohe Varianzaufklärung hat aber - wie im Falle des Modells für den 
Chloranreicherungsfaktor - nicht immer einen geringen Modellfehler (RMSE) zur Folge. Vor allem im Verhältnis zu den Mittelwerten der abhängigen Variablen (s.

Variationskoeffizient C.V.) wird deutlich, daß die Unsicherheit des Regionalisierungsmodells für den Anreicherungsfaktor von Chlor relativ hoch ist. Ein Variationskoeffizient von $28,1 \%$ steht hier in krassem Gegensatz zu dem höchsten Bestimmtheitsmaß von knapp $95 \%$ ! 


\section{Diskussion}

Die Ergebnisse, die in diesem Kapitel diskutiert werden, sind statistische Modelle. Sie beschreiben den Zusammenhang zwischen einer Zielvariable (z. B. durchschnittliche Niederschlagssumme im Januar in den Jahren 1961 bis 1990) und einer oder mehreren räumlichen Variablen. Wie genau sind die Modelle - auch im Verhältnis zur Datengenauigkeit? Sind die Modelle zu kompliziert oder gar zu einfach? Wie stark ist die Gültigkeit der Modelle durch Repräsentanzmängel des jeweils zugrundeliegenden Datenkollektivs eingeschränkt?

\subsection{Klimaelemente}

Drei Klimaelemente wurden regionalisiert: Durchschnittliche Lufttemperatur, durchschnittliche Niederschlagssumme und durchschnittliche relative Luftfeuchte für den Zeitraum von 1961 bis 1990. Im wesentlichen stützen sich die Ergebnisse auf Daten des Deutschen Wetterdienstes (DWD). Zwei zusätzliche Datenkollektive konnten im Bergland für die Regionalisierung der Lufttemperatur bzw. der Niederschlagssumme hinzugezogen werden. Im Falle des Niederschlages folgten auf eine schrittweise lineare Regression der Zielvariablen geostatistische Analysen zur Beschreibung der räumlichen Autokorrelation der Regressionsresiduen.

Die Regionalisierung der drei Klimaelemente erfolgte getrennt nach Berg- und Tiefland (Kap. 4.1.2, Kap.4.1.3 und Kap.4.1.4). Dieses Vorgehen kann sowohl durch Überlegungen zur Plausibilität als auch jeweils durch eine Erhöhung der Präzision begründet werden, wobei der letztgenannte Vorteil nur nach vorheriger (zusätzlicher) Regionalisierung für das Gesamtgebiet quantifiziert werden kann. Den beiden Vorteilen einer Trennung steht somit der erhöhte Aufwand einer solchen getrennten Modellierung und der Verlust an Einfachheit gegenüber. Stets muß ein Kompromiß zwischen Einfachheit und Präzision gefunden werden, wozu Aspekte der Plausibilität und des Modellierungsaufwands herangezogen werden können. 
Die Erfahrung des Bearbeiters und die anderer Wissenschaftler und Veröffentlichungen zeigt, daß der Aufbau einer brauchbaren Datenbasis in einem Projekt - zumal in einem solchen mit GIS- Anwendungen - stets im Verhältnis zur Modellierung ungleich mehr Aufwand erfordert (z. B. ESRI, 1990). Die Erhöhung des Modellierungsaufwands durch Teilung der Datenkollektive erscheint demgegenüber vernachlässigbar gering.

\subsubsection{Lufttemperatur}

Die Modelle für das Temperaturdatenkollektiv Niedersachsen, das vom Deutschen Wetterdienst (DWD) zur Verfügung gestellt werden konnte, zeigen sowohl in Bezug auf die räumlichen Variablen als auch auf deren Anteil an der Varianzaufklärung eine größere Ähnlichkeit mit den für das Bergland ermittelten Modellen als mit denjenigen, die an die Tieflanddaten angepaßt wurden, obwohl das Tieflanddatenkollektiv (29 Stationen) größer ist als das für das Bergland (16 Stationen). Dieser Umstand läßt sich durch die höhere Varianz der Berglanddaten (s. Standardabweichungen in Tabelle 13, Seite 63) im Vergleich zu den Tieflanddaten erklären (vgl. Standardabweichungen in Tabelle 18, Seite 69). Die Modelle für die durchschnittlichen Lufttemperaturen in Niedersachsen werden folglich durch die Meßergebnisse des Berglandes maßgeblich beeinflußt. Eine Trennung der Daten in ein Bergland- und ein Tieflanddatenkollektiv hatte daher vor allem eine Erhöhung der Präzision für die Tieflanddaten zur Folge. Zudem wurden räumliche Variablen mit großem Erklärungspotential für die Lufttemperatur im Tiefland gefunden, die ohne diese Maßnahme nicht ermittelt worden wären. In den folgenden Kapiteln sollen nunmehr die aus Gründen der Präzision und Plausibilität favorisierten Ergebnisse für die Teilgebiete diskutiert werden.

\subsubsection{Lufttemperatur im Bergland}

Zur Regionalisierung der Lufttemperatur im Bergland wurden Daten aus zwei Quellen verwendet. Durchschnittliche monatliche und jährliche Lufttemperaturen über den Zeitraum von 1961 bis 1990 konnte der Deutsche Wetterdienst (DWD) zur Verfügung stellen. Diese wurden vom DWD gemessen, um Aussagen zu den atmosphärischen Verhältnissen über dem beprobten Gebiet machen zu können. Die 
Problematik der Probenahme durch Laien und der damit verbundenen Möglichkeit von Stationsverlegungen wurde in Kap. 4.1.1 dargestellt. Die Berücksichtigung unterschiedlicher Meßsituationen während der Beobachtungsperiode erfolgte durch zeitgewichtete räumliche Variablen. Sie sind vor allem in stark reliefiertem Gelände einer Beschreibung ausschließlich der letzten Meßsituation vorzuziehen.

Eine Analyse des DWD-Datenkollektivs zeigte, daß die Aufklärung der Temperaturvarianz im Bergland schon alleine durch die Geländehöhe zum weitaus größten Teil geleistet wird.

Abbildung 33: Gegenüberstellung der durchschnittlichen Lufttemperatur im Dezember des DWD-Berglanddatenkollektivs und der Geländehöhe an den Meßstationen und das angepaßte lineare Regressionsmodell: temp $_{\text {Dez }}=22,63+(-0,051514) *$ Geländehöhe

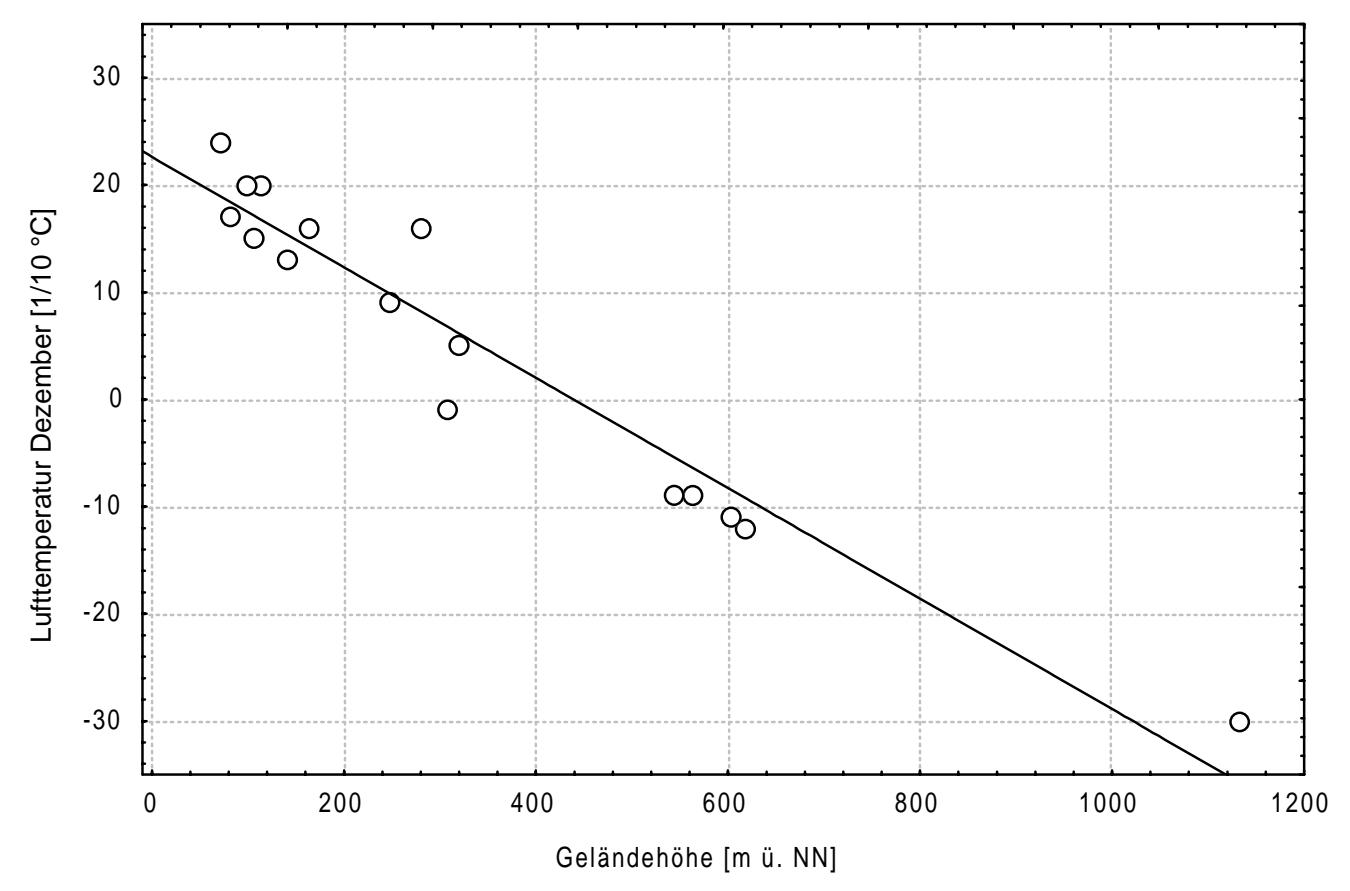

Alle Bestimmtheitsmaße der Regressionsmodelle liegen über $93 \%$ (s. Tabelle 44, S. 109). Der rooted mean squared error (RMSE) liegt mit einem Maximum von $0,4{ }^{\circ} \mathrm{C}$ weit unter dem Minimum der Standardabweichung der gemessenen Temperaturen von $1,4^{\circ} \mathrm{C}$ (s. Tabelle 13) und nur knapp über der Meßgenauigkeit von $1 / 10^{\circ} \mathrm{C}$. Bei einer Modellierung mit derart hoher Präzision erscheint die Integration einer weiteren Variablen ins Modell nicht sinnvoll und bei der gewählten Schranke von einem zusätzlichen Beitrag zum Bestimmtheitsmaß von mindestens $5 \%$ zudem kaum möglich. Einfachheit und Präzision des statistischen Modells sind gleichermaßen gegeben. 
Auch das Kriterium der Plausibilität der Modelle ist erfüllt. In der Literatur ist der Zusammenhang zwischen Lufttemperatur und Geländehöhe ausführlich beschrieben (z. B. HäCKEL, 1990) und wurde auch mehrfach zur Regionalisierung genutzt (Vent-Schmidt, 1985, Felbermeier, 1994, Böhner, 1996, Blennow \& Persson, 1998 und STEINER \& LEXER, 1998).

In einigen der aufgeführten Untersuchungen wurden zusätzlich zur Geländehöhe andere räumliche Variablen wie sky view factor (BLENNOW \& PERSSON, 1998; ähnelt der Relativen Exponiertheit), flowaccumulation (LAUGHLIN \& KALMA, 1990) oder auch Parameter der geographischen Lage (VENT-SCHMIDT, 1985, FELBERMEIER, 1994, BÖHNER, 1996) zur Regionalisierung der Lufttemperatur verwendet. Ähnliche, bedeutende Zusammenhänge zwischen der Lufttemperatur und anderen zur Verfügung stehenden räumlichen Variablen konnten für das DWD-Datenkollektiv nicht gefunden werden (s.o.). Vor dem Hintergrund erheblicher Repräsentanzmängel der DWD-Stationen wurde daher das zweite zur Verfügung stehende Datenkollektiv TRANSEKT mit in die Untersuchungen einbezogen (s. Kap.4.1.2.1), um die Bedeutung anderer räumlicher Variablen für die Verteilung der Lufttemperatur zu überprüfen. Dies geschah, obwohl den beiden Datenkollektiven unterschiedliche Beobachtungszeiträume zugrunde liegen. Eine Kombination der beiden Datenkollektive führt $\mathrm{zu}$ einer Verbesserung der Repräsentanz für das Untersuchungsgebiet.

Die Daten des TRANSEKT-Datenkollektivs zeigten für alle Monate bis auf den Juli einen signifikanten Zusammenhang zwischen der Zielvariablen Lufttemperatur und der räumlichen Variable QWKaltluftabflu $\beta$ (Kap. 3.3.6). Andere Variablen konnten nicht in dem geforderten Maß zur Aufklärung der Lufttemperaturvarianz beitragen.

HARTMANN \& SCHNELLE (1970) beschreiben u. a. die Bildung von Kaltluftseen als wichtigen Einflußfaktor für die Lufttemperatur. Zum Einfluß der Strahlungsintensität auf die Lufttemperatur bemerken sie, daß dieser durch Winde schnell aufgelöst werden kann. LAUGHLIN \& KALMA (1990) regionalisierten die Minimumtemperatur innerhalb eines Zielgebiets in Süd-Ost-Australien mit Hilfe der Minimumtemperatur einer im Zielgebiet gelegenen Temperaturmeßstation und der Geländehöhe. Die Restvarianz konnte zu $65 \%$ durch die Varianz eines Indexes für flowaccumulation bzw. catchment area, der dem hier genutzten Index für Kaltluftabfluß gleicht, aufgeklärt werden. Blennow und Persson (1998) konnten diesen Zusammenhang 
nicht bestätigen, was sie auf die kleinflächigen Unterschiede der Waldbestände zurückführten, die eine Sammlung oder einen Fluß von Kaltluft behindern.

Der Index für Kaltluftabflu $\beta$ bzw. seine Quadratwurzel QWKaltluftabflu $\beta$ beschreiben die Exponiertheit eines Standortes für Kaltluftabfluß. Ein Standort mit hohen Werten sollte eine geringere Tagesdurchschnittstemperatur haben als einer mit niedrigen Werten. Kalte Luft fließt ähnlich wie eine zähe, breiartige Flüssigkeit (HÄCKEL, 1990, GEIGER, 1961). Daß ein solcher breiartiger Kaltluftfluß durch die Analyse des TRANSEKT-Datenkollektivs gefunden wird, durch die Analyse der DWD-Daten aber nicht bestätigt werden kann, kann auf die Lage der DWDMeßstationen zurückgeführt werden. Da diese durch ehrenamtliche Mitarbeiter zumeist in der Nähe ihrer Wohnungen betreut werden, ist es wahrscheinlich, daß meßbarer Kaltluftabfluß durch die Bebauung und Bepflanzung in der Umgebung der Meßstationen in bedeutendem Maß verhindert oder beeinträchtigt wird. Zudem führen die unten noch für die Landnutzungsvariable Bebauung zu besprechenden Effekte (geringere Transpiration, geringere nächtliche Ausstrahlung durch Horizontüberhöhungen und höhere Wärmeleitfähigkeit der Oberflächen) in Siedlungen $\mathrm{zu}$ einer geringeren Wahrscheinlichkeit von Kaltluftbildung und Kaltluftabfluß.

Der im TRANSEKT-Datenkollektiv gefundene Zusammenhang zwischen Lufttemperatur und $Q W$ Kaltluftabflu $\beta$ scheint somit durch logische Überlegungen und die Ergebnisse anderer Untersuchungen gestützt. $\mathrm{Zu}$ diskutieren ist nunmehr die Art und Weise, wie der gefundene Einfluß in dem Regionalisierungsmodell für das Bergland berücksichtigt wurde. Zunächst ist $\mathrm{zu}$ unterstreichen, daß die Beobachtungsperiode des TRANSEKT-Datenkollektivs (1994 bis 1995) von der des DWD-Datenkollektivs (1961 bis 1990) abweicht. Eine Gleichstellung beider Kollektive und eine gemeinsame Analyse der Daten, die diesen Umstand nicht berücksichtigt, darf daher nicht gewählt werden. Ziel muß es vielmehr sein, die gewünschte Beobachtung des TRANSEKT-Datenkollektivs zu integrieren, ohne dadurch die besonderen Verhältnisse der beiden Beobachtungsjahre auf den gesamten Regionalisierungszeitraum zu übertragen.

Um ausschließlich denjenigen Informationsgehalt in das Regionalisierungsmodell zu integrieren, der im DWD-Datenkollektiv nicht oder zu schwach ausgeprägt ist, wurde die in Kap. 5.1.1.1 beschriebene Modellierung gewählt (s.a. Tabelle 45). Der 
Regressionskoeffizient für $Q$ WKaltluftabflu $\beta$ wird dabei durch beide Datenkollektive parametrisiert, die additive Konstante und der Regressionskoeffizient der Geländehöhe werden ausschließlich mit den DWD-Daten parametrisiert. Dies spiegelt die Überzeugung wieder, daß beide Datenkollektive Erkenntnisse über den Zusammenhang zwischen durchschnittlicher Temperatur und QWKaltluftabflu $\beta$ ermöglichen, obwohl nur die Analyse des TRANSEKT-Datenkollektivs einen bedeutenden Zusammenhang zeigt. Der in den Jahren 1994 und 1995 vom Beobachtungszeitraum 1961 bis 1990 abweichende Zusammenhang zwischen Lufttemperatur und Geländehöhe sowie eventuelle Abweichungen von der durchschnittlichen Lufttemperatur im Beobachtungszeitraum werden durch die Integration von Kombinationen mit einer Dummy-Variable (TRA $\times$ Geländehöhe bzw. TRA) aus dem Regionalisierungsmodell herausgehalten. Eine ausschließliche Parametrisierung des Regressionskoeffizienten für $Q$ WKaltluftabflu $\beta$ mit Hilfe des TRANSEKT-Datenkollektivs wurde nicht gewählt, da neben der kurzen Beobachtungsdauer auch Repräsentanzmängel bezüglich der Geländehöhe und der räumlichen Verteilung der Stationen bestehen (Kap. 4.1.2.1, S. 66ff).

Sowohl die Bestimmtheitsmaße als auch die Werte des RMSE zeigen eine sehr gute Anpassung der Modelle an die Daten und lassen einen geringen Regionalisierungsfehler erwarten. Dies gilt um so mehr, als durch die Verknüpfung der beiden Datenkollektive die Basis der untersuchten Standorte und damit die Repräsentanz der Modelle erweitert wurde.

\subsubsection{Lufttemperatur im Tiefland}

Für die Regionalisierung der Lufttemperatur im niedersächsischen Tiefland stand ein DWD-Datenkollektiv mit den Meßwerten von 29 Stationen zur Verfügung (Kap. 4.1.2.2). Der weitaus größte Teil des niedersächsischen Tieflandes wird durch die Stationen repräsentiert, nur extreme orographische Meßsituationen wurden vom DWD gemieden.

Das Bestimmtheitsmaß der monatlichen Modelle liegt allgemein über $80 \%$. Nur das Modell für die durchschnittliche Lufttemperatur im August unterschreitet diese Marke deutlich $(65,1 \%)$, kann aber immer noch als zufriedenstellend angesehen werden. Auch die Werte des RMSE sind mit einem Maximum von 0,23 ${ }^{\circ} \mathrm{C}$ nur ca. doppelt so groß wie die Meßgenauigkeit, die der DWD für seine Stationen vorsieht 
(DWD, 1980). Die Standardabweichungen der Meßwerte liegen zwischen 0,33 ${ }^{\circ} \mathrm{C}$ (August) und $0,58{ }^{\circ} \mathrm{C}$ (Januar) und damit deutlich über den Werten des RMSE. Der Modellfehler liegt somit deutlich unter demjenigen einer Regionalisierung durch Mittelwertangabe. Die Modelle erfüllen somit das Kriterium der Präzision.

Im Tiefland kann nur ein geringer Teil der Varianz der Lufttemperatur durch die höhenabhängiger Variablen aufgeklärt werden. Während im Bergland die Geländehöhe das mit Abstand größte Erklärungspotential für die Lufttemperatur hat, zeigen im Tiefland die Exponiertheit zum Umgebungsminimum (April bis Juli) und der Lee-Effekt 25 (März und August) einen bedeutenden Zusammenhang mit der Lufttemperatur (s. Abbildung 26). Neben diesem Unterschied $\mathrm{zu}$ den Berglandmodellen ist auch die Integration der Landnutzungsvariablen Bebauung und Gewässer und der lageabhängigen Variablen Rechtswert, Hochwert und Abstand zur Küste hier zu diskutieren.

Ähnliche räumliche Variablen wie die hier verwendete höhenabhängige Variable Exponiertheit zum Umgebungsminimum wurden von BLENNOW \& PERSSON (1998) zur Regionalisierung der Lufttemperatur verwendet. Namentlich der vorher bereits von DOZIER \& FREW (1990) beschriebene sky view factor und die Höhendifferenz zu einem Umgebungsminimum (Radius: $200 \mathrm{~m}$ ) gleichen als Kombination der hier verwendeten Variablen. BLENNOW \& PERSSON (1998) ermittelten für die Höhendifferenz zum Umgebungsminimum einen positiven Regressionskoeffizienten, der vor dem Hintergrund der Meßbedingungen plausibel erscheint (Inversionslagen in fünf klaren und windstillen Nächten). Für die durchschnittliche monatliche bzw. jährliche Lufttemperatur muß hingegen von einer negativen Korrelation ausgegangen werden, da hohe Werte der relativen Höhe eine erhöhte nächtliche Ausstrahlung und durch Windexponiertheit erhöhte Transpiration erwarten lassen. Die Exponiertheit zum Umgebungsminimum ist definitionsgemäß eine Variante der Relativen Exponiertheit (ähnlich sky view factor) durch Multiplikation mit der relativen Höhe (Kap. 3.3.5). Da beide Einflußfaktoren negative Korrelation mit der durchschnittlichen Lufttemperatur erwarten lassen, mußte dies auch für ihre Kombination, die Exponiertheit zum Umgebungsminimum gefordert werden. Dies wird durch die monatlichen Modelle für April bis Juli erfüllt.

Die Regressionskoeffizienten der zweiten höhenabhängigen Variablen, Lee-Effekt 25, sind in beiden Modellen (März und August) positiv. Dies zeigt höhere 
Lufttemperaturen für solche Stationen an, die östlich von Geländeerhebungen gelegen sind. Da Westen in diesem Gebiet die häufigste Windrichtung ist (Häckel 1990, S. 288f), kann auch formuliert werden: Diejenigen Stationen, die im Windschatten von Geländeerhöhungen liegen, messen höhere Lufttemperaturen als andere. Dieser Zusammenhang läßt sich durch die adiabatische Erwärmung von fallenden Luftpaketen (Föhn- Effekt) erklären. Es handelt sich folglich um einen plausiblen, wenn auch nur in den Monaten März und August bedeutenden, Zusammenhang.

Bis auf das Modell zur Beschreibung der Verteilung der Lufttemperatur im August ist an jedem monatlichen und an dem jährlichen Modell jeweils eine lageabhängige Variable beteiligt (Tabelle 46, S. 112). Am häufigsten konnte der Rechtswert einen bedeutenden Teil der Varianz der Lufttemperatur erklären. Die negativen Regressionskoeffizienten zeigen, daß in östlichen Gebieten in den jeweiligen Monaten (September bis März) und im Jahr durchschnittlich tiefere Temperaturen herrschen. Betrachtet man die Lage Niedersachsens mit der Nordsee (Golfstrom!) in westlicher und nördlicher Richtung, so ist dieses Ergebnis durchaus plausibel.

Die Abnahme der durchschnittlichen Lufttemperatur mit zunehmendem Hochwert in den Monaten April und Mai kann einerseits durch geringere Strahlungsintensitäten nördlicher Breitengrade - begründet werden, andererseits aber auch in den Frühjahrsmonaten April und Mai durch die schnellere Erwärmung küstenferner Regionen bzw. durch den ausgleichenden, kühlenden Effekt des Meeres. Analog können höhere Temperaturen von Stationen mit größerem Abstand zur Küste in den Monaten Juni und Juli plausibel erklärt werden.

Der gegenläufige Effekt wird in den Monaten August bis Dezember durch die Landnutzungsvariable Gewässer beschrieben: In diesen Monaten wurden an Stationen mit hohem Anteil an Gewässern (Kap. 3.4.1) höhere Temperaturen gemessen als an Stationen in Gebieten mit geringerem Gewässeranteil. Der ausgleichende Effekt führt nun zur langsameren Abkühlung in Gebieten mit hohem Gewässeranteil, wozu der Küstenraum zu zählen ist.

Die geringere nächtliche Abkühlung in Siedlungen durch geringere Abstrahlung (BLENNOW \& PERSSON, 1998 zum sky view factor oder HÄCKEL, 1990, S. 278), die niedrigere Transpiration und die höhere Dichte und Wärmeleitfähigkeit versiegelter Oberflächen (GRAVENHORST, mdl. Mitteilung 1999, HÄCKEL, 1990, S. 282) sind 
bekannt. Für die Landnutzungsvariable Bebauung wird dies mit positiven Regressionskoeffizienten in den Modellen für die Monate Februar bis Oktober und für die durchschnittliche Jahrestemperatur bestätigt.

Insgesamt läßt sich die Varianz der Lufttemperatur im niedersächsischen Tiefland folglich zu einem großen Teil durch die Varianzen von Variablen beschreiben, die vier verschiedene Effekte beschreiben: Einfluß der Kontinentalität an den Standorten (Rechtswert, Hochwert, Abstand z. Küste und Gewässer), Erwärmung durch adiabatische Effekte (Lee-Effekt 25), Abkühlung durch Windexponiertheit bzw. Transpiration und nächtliche Abstrahlung (expmin) und Erwärmung bzw. geringere Abkühlung in anthropogen geprägtem Gelände (Bebauung). Alle diese Variablen haben plausible Regressionskoeffizienten und bilden zusammen einfache Modelle hoher Präzision.

\subsubsection{Niederschlagssumme}

In der Diskussion der Regionalisierung monatlicher und jährlicher durchschnittlicher Niederschlagssummen muß neben der Aufspaltung in ein Bergland- und ein Tieflanddatenkollektiv auch die Erweiterung der Datenbasis durch die Integration eines zusätzlichen Datenkollektivs (hier: Harzwasserwerke, HWW) diskutiert werden. Diese Erweiterung der Datenbasis sowie die Berglangmodelle selbst werden im folgenden Unterkapitel diskutiert. Die Diskussion der Tieflandmodelle erfolgt in Kapitel 6.1.2.2. Zusätzlich zu den Ergebnissen der schrittweisen Regressionsanalyse sind zudem für beide Untergebiete die Ergebnisse der geostatistischen Analyse zu besprechen.

Zunächst soll nun die Teilung der DWD-Daten nach Zugehörigkeit zu Berg- bzw. Tiefland besprochen werden. So wie im Fall der durchschnittlichen Lufttemperatur kann auch für die gemeinsamen Modelle der monatlichen und jährlichen Niederschlagssummen (Tabelle 47, S. 115) eine größere Ähnlichkeit mit den Modellen des Berglandes (Tabelle 49, S. 117) als mit denen des Tieflandes (Tabelle 55, S. 126) festgestellt werden. Zwar ist an den meisten Modellen des Gesamtdatenkollektivs auch eine Variable der geographischen Lage (Abstand zur Küste) beteiligt, aber deren Anteil an der Varianzaufklärung entspricht nicht dem der geographischen Variablen in den Tieflandmodellen (vgl. Abbildung 30, S. 127). Die 
Frage, ob die Dominanz der Berglandmodelle zu schlechterer Anpassung im flächenmäßig größeren Tiefland führt, kann Durch den in Tabelle 48 (S. 116) angestellten Vergleich von Summen quadrierter Residuen kann gezeigt werden, daß die Dominanz der Berglanddaten zu schlechterer Anpassung im flächenmäßig größeren Tiefland führt. Die Tieflanddaten werden grundsätzlich immer genauer durch ein eigenes Modell beschrieben werden als durch ein gemeinsames für ganz Niedersachsen. Dieses Bild ist bei den Berglanddaten nicht ebenso eindeutig, was aber auch angesichts der Ähnlichkeiten mit den Gesamtmodellen zu erwarten ist. Dennoch kann auch für die Berglanddaten beobachtet werden, daß ein einfacheres Teilkollektivmodell einem komplizierteren Gesamtmodell in Bezug auf die Präzision überlegen ist (Oktober), der umgekehrte Fall tritt jedoch nicht ein. Eine getrennte Modellierung ermöglicht folglich - vor allem für das Tiefland - eine präzisere Beschreibung der Daten und verspricht daher einen geringeren Regionalisierungsfehler.

\subsubsection{Niederschlag im Bergland}

Zur Regionalisierung der monatlichen und jährlichen Niederschlagssummen im Bergland standen zwei Datenquellen zur Verfügung. Die Daten der Harzwasserwerke (HWW) beschränken sich auf Stationen im niedersächsischen Harz. Die Meßnetzdichte des HWW übertrifft die des Deutschen Wetterdienstes (DWD; s. Abbildung 17 und Abbildung 18, S. 73f). Die Tatsache, daß der DWD einige HWW-Stationen in sein Meßnetz aufgenommen hat ${ }^{5}$, verdeutlicht die Vergleichbarkeit der Meßmethodik. Auch der Beobachtungszeitraum der beiden Datenkollektive stimmt überein (Kap. 4.1.3.1). Es ist um so erstaunlicher, daß die in Kap. 5.1.2.1 dargestellten Ergebnisse Unterschiede der in den Daten enthaltenen Zusammenhänge belegen. Neben der Diskussion der Ergebnisse von schrittweiser Regression und Geostatistik wird daher die Problematik der gemeinsamen Analyse der beiden Datenkollektive ein Schwerpunkt der Diskussion sein.

Die Modelle der schrittweisen linearen Regressionen für das Berglanddatenkollektiv des DWD setzen sich aus vier verschiedenen räumlichen Variablen zusammen: Geländehöhe, Leewirbel, Luv und Hochwert. Die Variable mit dem mit Abstand

\footnotetext{
5 im Rahmen dieser Arbeit als DWD-Stationen geführt
} 
größten Erklärungspotential war die Geländehöhe. Zusammen mit einer der beiden Windeffekt-Variablen und im August zusammen mit dem Hochwert wurden Modelle mit Bestimmtheitsmaßen zwischen $76 \%$ und $88 \%$ gefunden, der RMSE der Modelle liegt bei $35 \%$ bis $48 \%$ der Standardabweichungen der Meßwerte.

Die WMO (1972) (aus: KLEIN, 1994) gibt als Einflußfaktoren für die Regionalisierung von Niederschlägen „die Großwetterlage, die geographische Breite, die Entfernung zum Meer, die Höhenlage über dem Meer sowie Luv- und LeeEffekte durch die Topographie“ an. Diese Zusammenstellung entspricht bis auf die Großwetterlage, deren Betrachtung bei der Regionalisierung langjähriger Mittelwerte nicht sinnvoll ist, den Zusammenhängen, die für die untersuchten Datenkollektive gefunden wurden.

Für alle DWD-Berglandmodelle und für die meisten Modelle des integrierten DWD/HWW-Datenkollektivs wurde eine Abhängigkeit der Niederschlagssumme von der Geländehöhe gefunden. Ein solcher Einfluß wurde auch vom ARBEITSKREIS StANDORTKARTIERUNG (1985), von FELbERMEIER (1994), KLEIN (1994), SABOROWSKI \& STOCK (1994), BÖHNER (1996) und von STEINER \& LEXER (1998) beschrieben. Dabei wurde dieser Zusammenhang nicht immer linear modelliert. SABOROWSKI \& STOCK (1994) fanden eine bessere Beschreibung der ihnen zur Verfügung stehenden DWD-Daten aus dem niedersächsischen Harz durch die Verwendung des natürlichen Logarithmus der Geländehöhe. Eine solche Transformation erschien wegen erheblicher Unterschätzungen im oberen Meßwertbereich hier nicht angebracht.

Neben der Geländehöhe konnten zwei weitere orographische Variablen einen bedeutenden Teil der Niederschlagsvarianz im DWD-Datenkollektiv aufklären: Leewirbel und Luv. Beide unterscheiden sich in ihrer Berechnung lediglich durch die Richtung der betrachteten Kreisausschnitte (Kap. 3.3.3 bzw. Kap. 3.3.4). Während in den Wintermonaten vor allem an solchen Standorte höhere Niederschläge gemessen worden sind, die östlich von Geländeerhebungen gelegen sind (Leewirbel), waren dies in den Sommermonaten Stationen westlich von Höhenzügen oder Bergen ( $L u v)$. Entsprechend der Berechnung der Variablen bedeutet dies für die Art der Niederschlagsereignisse einen größeren Anteil von Steigungsregen im Sommer und/oder eine verstärkte Wirksamkeit von Leewirbeln im Winter, was durch den hohen winterlichen Anteil von Schneefällen an den Niederschlägen im 
niedersächsischen Bergland plausibel erscheint. Der Schnee ist durch seine größere spezifische Oberfläche mehr als Niederschläge anderer Form dem Wind und damit der Verwirbelung ausgesetzt.

Eine ähnliche Modellierung der topographisch bedingten Niederschlagssituation erfolgte durch FELBERMEIER (1994). Über die Summe von Geländeerhebungen in verschiedenen Himmelsrichtungen oder Rauhigkeitsparameter und mittlere Steigung wurden die Anströmungsverhältnisse modelliert. Er verwendete auch Kombinationen und Transformationen dieser Parameter, was eine Interpretation der von ihm gefunden Zusammenhänge erschwert. KLEIN (1994) beschreibt den Zusammenhang verschiedener Relieftypen mit dem Niederschlag. Allerdings sind auch diese Relieftypen nur eingeschränkt zu unmittelbarer Interpretation geeignet.

Der Hochwert konnte als Variable der geographischen Lage für den Monat August einen bedeutenden Teil zur Varianzaufklärung der Niederschlagssumme beitragen. In anderen Monaten führt die Integration des Hochwerts in das jeweilige Niederschlagsmodell (of nur knapp) nicht $\mathrm{zu}$ einer Erhöhung des Bestimmtheitsmaßes um $5 \%$. Wie bereits oben beschrieben, wurden von der WMO (1972) (aus: KLEIN, 1994) der Breitengrad und die Entfernung zum Meer, die für das niedersächsische Bergland dieser Variable sehr ähnelt, als mögliche Einflußgrößen auf den Niederschlag genannt. Auch der Arbeitskreis StandortKartierung (1985) nennt den Abstand zur Küste als Einflußfaktor für die Höhe des Niederschlages. Der in den Daten gefundene Zusammenhang erscheint somit plausibel.

Die Residuen der DWD-Regressionsmodelle waren Basis einer geostatistischen Analyse (Kap. 5.1.2.1). Die für die Regressionsresiduen in den Abstandsklassen ermittelten empirischen Semivariogramme und die an sie angepaßten theoretischen Semivariogramme des sphärischen Typs (s.a. Kap. 2.3) konnten durch wenige iterative Regressionen nach der Methode der generalisierten kleinsten Quadrate (s. Kap. 2.5) bis zu der in Tabelle 50 auf Seite 119 aufgeführten Form optimiert werden. Hierdurch wurden ohne unverhältnismäßig großen Aufwand bedeutende Verbesserungen der Schätzungen erreicht, wie aus den Ergebnissen der Kreuzvalidierung hervorgeht (Tabelle 51, S. 121).

KLEIN (1994) verweist auf die Möglichkeit, mit der Methode des Residuen-Kriging die Differenz zwischen Meßwert und Regressionswert, das Residuum, ausgleichen 
zu können. Die große Bedeutung dieses Verfahrens in der Regionalisierung liegt jedoch weniger in der Korrektur der Vorhersage an den Stützpunkten als vielmehr in der Objektivierung der Interpolation für die restliche Fläche des Zielgebietes. Während z. B. bei einer distanzgewichteten Interpolation die Entscheidung nach der Art der Gewichtung (linear, quadratisch, ...) dem Empfinden des Bearbeiters überlassen bleibt, wird die Gewichtung im Falle des Kriging durch das Semivariogramm definiert. Dieses muß an empirische Werte angepaßt werden. Die Interpolationsmethode Kriging entzieht die Entscheidung, wie die Distanz zwischen Zielpunkten und Stützstellen gewichtet werden soll, weitestgehend subjektiven Einflüssen und führt zu einer Optimallösung, was den Unterschied und Vorteil anderen Interpolationsmethoden gegenüber ausmacht.

Ein Nachteil der Regionalisierung unter Verwendung des geostatistischen Verfahrens Kriging ist die Verwendungsbeschränkung der Ergebnisse auf Anwender, denen entsprechendes Datenmaterial und die geeignete Soft- und Hardware zur Verfügung stehen. Die Ergebnisse einer Regionalisierungsfunktion, die auf einer ausschließlichen Regression z. B. unter Einbeziehung der Koordinaten, der Geländehöhe und einfacher abgeleiteter räumlicher Variablen beruht, sind vor Ort reproduzierbar oder zumindest abschätzbar. Diese Einschränkung bezüglich der Anwendbarkeit in der Praxis kann jedoch angesichts fortschreitender Digitalisierung und Verfügbarkeit räumlicher Daten seitens amtlicher und privater Stellen nur zeitlich begrenzt gelten. Eine Verbreitung von räumlich relevanten Ergebnissen ist schon jetzt zumindest technisch über das Internet möglich.

Für das integrierte DWD/HWW-Datenkollektiv wurden auf dem Weg der schrittweisen linearen Regression Modelle parametrisiert (Tabelle 52, S. 122), die es mit denen des reinen DWD-Datenkollektivs zu vergleichen gilt. Auch die Tatsache, daß eine weiterführende Semivariogrammanalyse nur eingeschränkt möglich war (s. S. 121f), soll diskutiert werden.

Der augenfälligste Unterschied der Parametrisierung beider Datenkollektive ist das Fehlen der Variable Luv und die Verwendung der Variable Lee-Effekt 50 in den Modellen des DWD/HWW-Datenkollektivs. Für die Monate September bis März wird für das HWW-Datenkollektiv zwar ein signifikanter Zusammenhang ermittelt, der zur Verbesserung des Bestimmtheitsmaßes von mehr als $5 \%$ gegenüber einem reinen Geländehöhenmodell führen würde, aber andere Variablen zeigen ein höheres 
Erklärungspotential (Anhang 10). Entsprechende Untersuchungen für die DWDStationen im Harz deuten zwar im Vergleich zum gesamten Bergland auf ein schwächeres Erklärungspotential des Luv-Effektes hin, die Variable Luv zeigt aber dennoch einen bedeutenden Zusammenhang zur Niederschlagssumme und wird zur Modellbildung herangezogen (Anhang 9).

Während es im DWD-Datenkollektiv Stationen mit niedrigem Wert der Variable Luv und niedrigem Wert der Variable Geländehöhe gibt, fehlen diese im HWWDatenkollektiv. Nur sehr hoch gelegene HWW-Stationen haben einen geringen Wert für Luv. An hoch gelegenen Stationen wird aber mehr Niederschlag gemessen als an niedrigen, weshalb für das HWW-Datenkollektiv sogar ein negativer Korrelationskoeffizient zwischen Niederschlag und $L u v$ ermittelt wird. Selbst im DWD-Datenkollektiv Harz sind Stationen mit niedriger Luv vorhanden, an denen auch wenig Niederschlag gemessen wurde. Verdeutlicht wird dies durch Abbildung 34:

Abbildung 34: Gegenüberstellung der durchschnittlichen Niederschlagssumme im Juli und der räumlichen Variable Luv für alle Berglandstationen

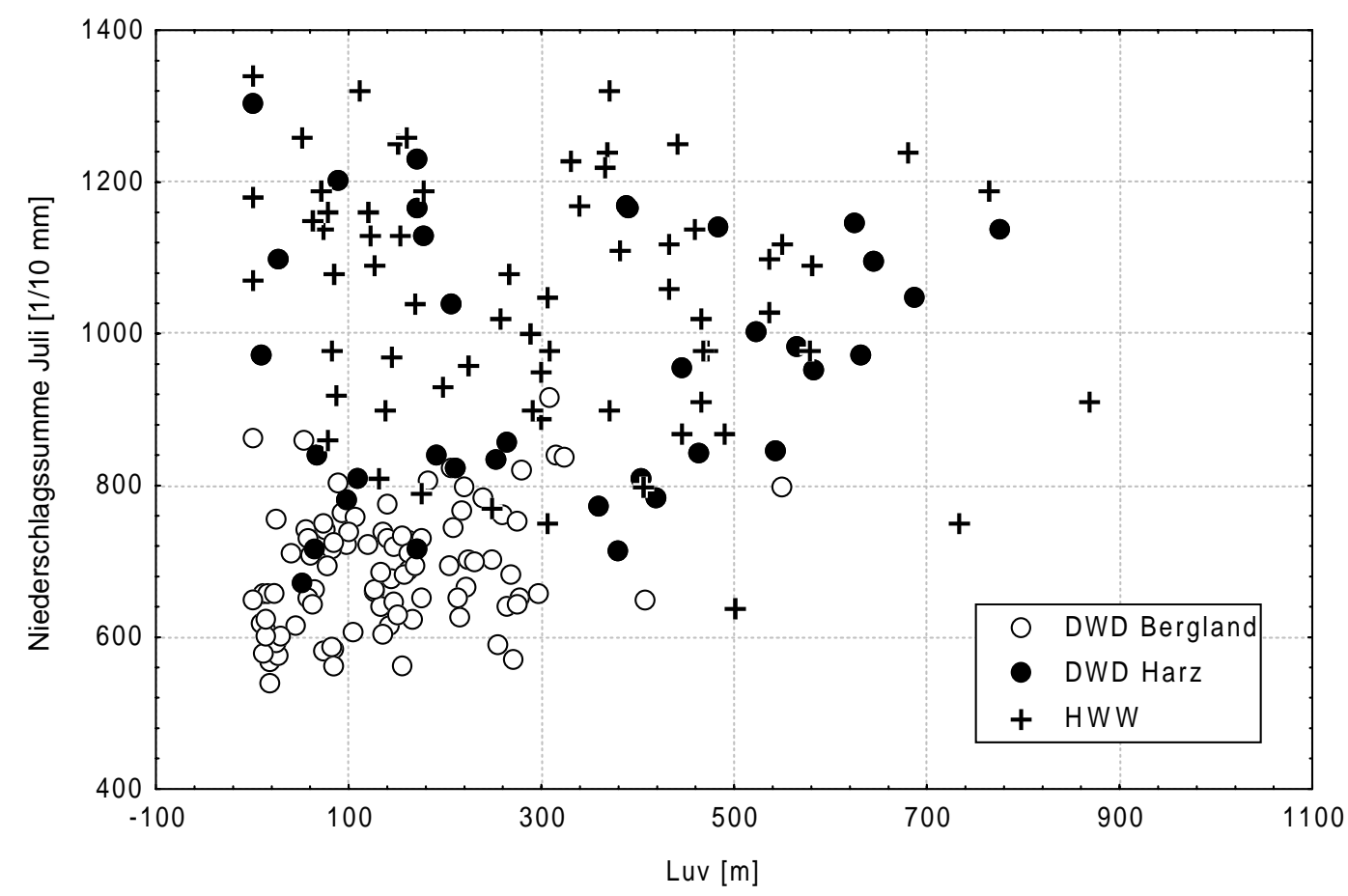

Aus Abbildung 34 geht deutlich hervor, daß die nicht im Harz gelegenen Stationen des DWD-Datenkollektivs bei niedrigen Werten für $L u v$ auch niedrige Niederschlagshöhen messen. Einige Stationen des HWW-Datenkollektivs zeigen bei hohen Werten für $L u v$ sogar niedrige Niederschläge. Dies kann durch die Lage 
entsprechender Stationen im Harz, im Windschatten anderer vorgelagerter Berge erklärt werden.

Bei der Berechnung der räumlichen Variablen Lee-Effekt 50 und Lee-Effekt 25 kommt es vor allem am Westrand des Untersuchungsgebietes zu Unsicherheiten (s.a. Kap. 3.3.3, S. 35). Dieses Problem wird jedoch als nicht bedeutend eingestuft und kann notfalls durch eine Modellbildung ohne Berücksichtigung dieser Variablen umgangen werden (s. Anhang 12). Ansonsten entsprechen die negativen Regressionskoeffizienten den in 3.3.3 gemachten Annahmen eines geringeren Niederschlages bei hohen Variablenwerten.

Zu klären ist die Frage, weshalb für das integrierte DWD/HWW-Datenkollektiv die Modellierung räumlicher Autokorrelation teilweise unmöglich und in den anderen Fällen schwieriger war als für das reine DWD-Datenkollektiv. Dies geschah durch eine Analyse der halben Differenzen zwischen zwei Meßwerten eines Paares mit Abstand $|\mathrm{h}|$. Diese können im Programm VARIOWIN 2.2, das zur Semivariogrammanalyse verwendet wurde, in einer sogenannten Variogrammwolke dargestellt werden (variogram cloud, Abbildung 35, S. 157). Durch einen Mausklick auf einen beliebigen Punkt dieser Abbildung wird das zugrundeliegende Stationspaar in einer parallel geöffneten Lageskizze der Stationen markiert, und die relevanten Werte der betroffenen Paare werden in einem gesonderten Fenster angezeigt (Abbildung 36). Dadurch ist eine übersichtliche Beurteilung der Lage interessanter Paare möglich. Der in Abbildung 35 eingefügte Pfeil zeigt auf eine Stelle, deren Auswahl mit der Maus das in Abbildung 36 dargestellte Bild bewirkt. Dieses zeigt folglich die Lage einiger Paare mit einem Abstand |h| von ca. 14,3 km bis 16,4 km, die relativ hohe Differenzen zeigen. Wie Abbildung 36 zu entnehmen ist, liegen alle zu diesen Paaren gehörenden Stationen im Harz. Auch die Paare, die in Abbildung 35 noch oberhalb der durch den Pfeil markierten Lage durch Kreise symbolisiert werden, liegen im Harz. Von vielen Paaren in diesem Abstandsbereich unterhalb der Pfeilspitze mit relativ hohen Differenzen liegt mindestens jeweils eine Station im Harz. 
Abbildung 35: Abbildung einer Variogrammwolke aus dem Modul Vario2D des Programmes VARIOWIN 2.2 für das DWD/HWW-Datenkollektiv, OLS- Residuen Juliniederschlag

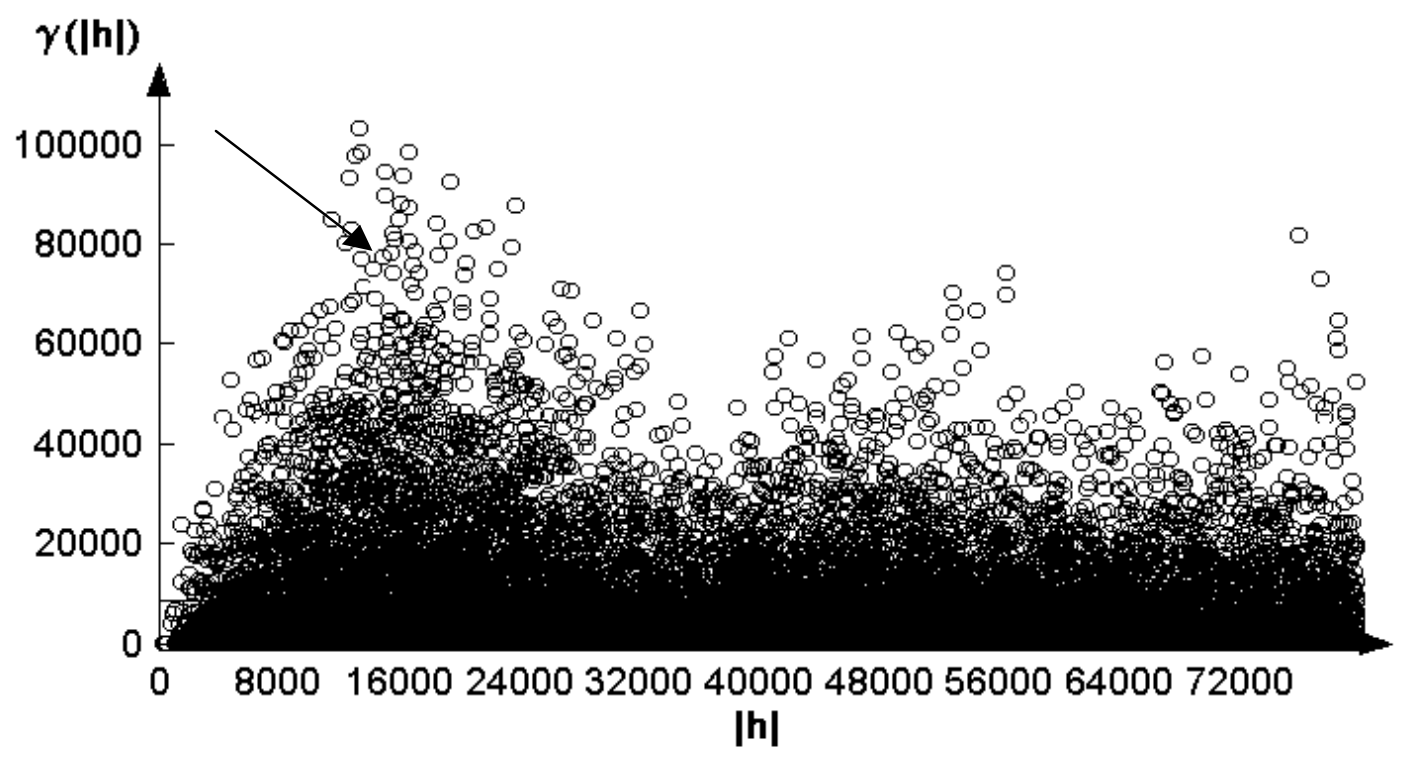

Abbildung 36: Markierung der per Mausklick ausgewählten Stationspaare (s. Pfeil in Abbildung 35) des DWD/HWW-Datenkollektivs, OLSResiduen Juliniederschlag

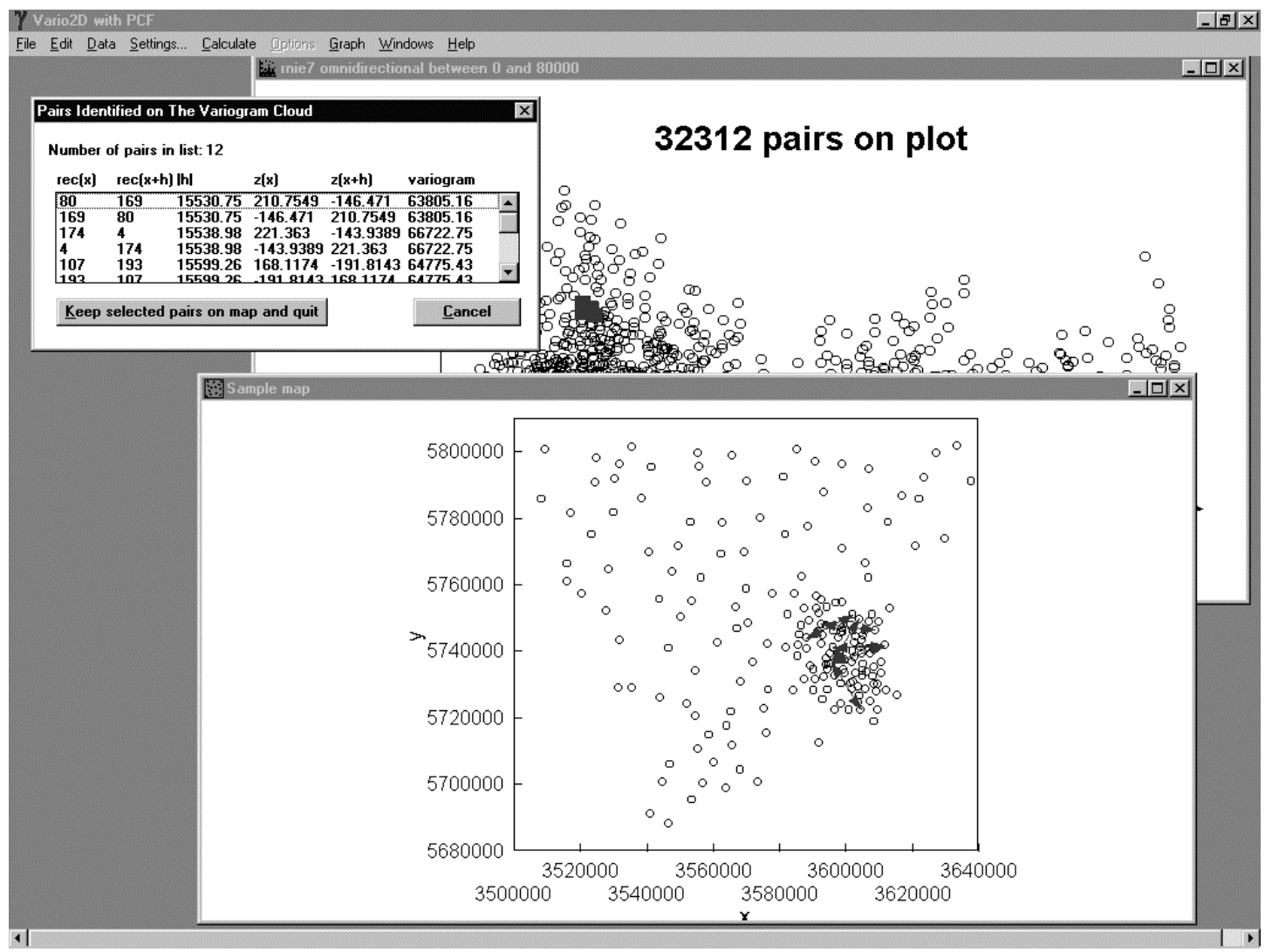

Zum Vergleich mit Abbildung 35 wird die entsprechende Semivariogrammwolke für das reine DWD-Datenkollektiv in Abbildung 37 dargestellt. Auffällig ist, daß im 
Abstandsbereich unter $25 \mathrm{~km}$ deutlich weniger große Differenzen zu verzeichnen sind. Auch in diesem Datenkollektiv liegen aber die Stationspaare mit auffällig hoher Differenz ganz oder mit zumindest einer Station im Harz.

\section{Abbildung 37: Variogrammwolke für das DWD-Datenkollektiv Bergland, OLS- Residuen Juliniederschlag}

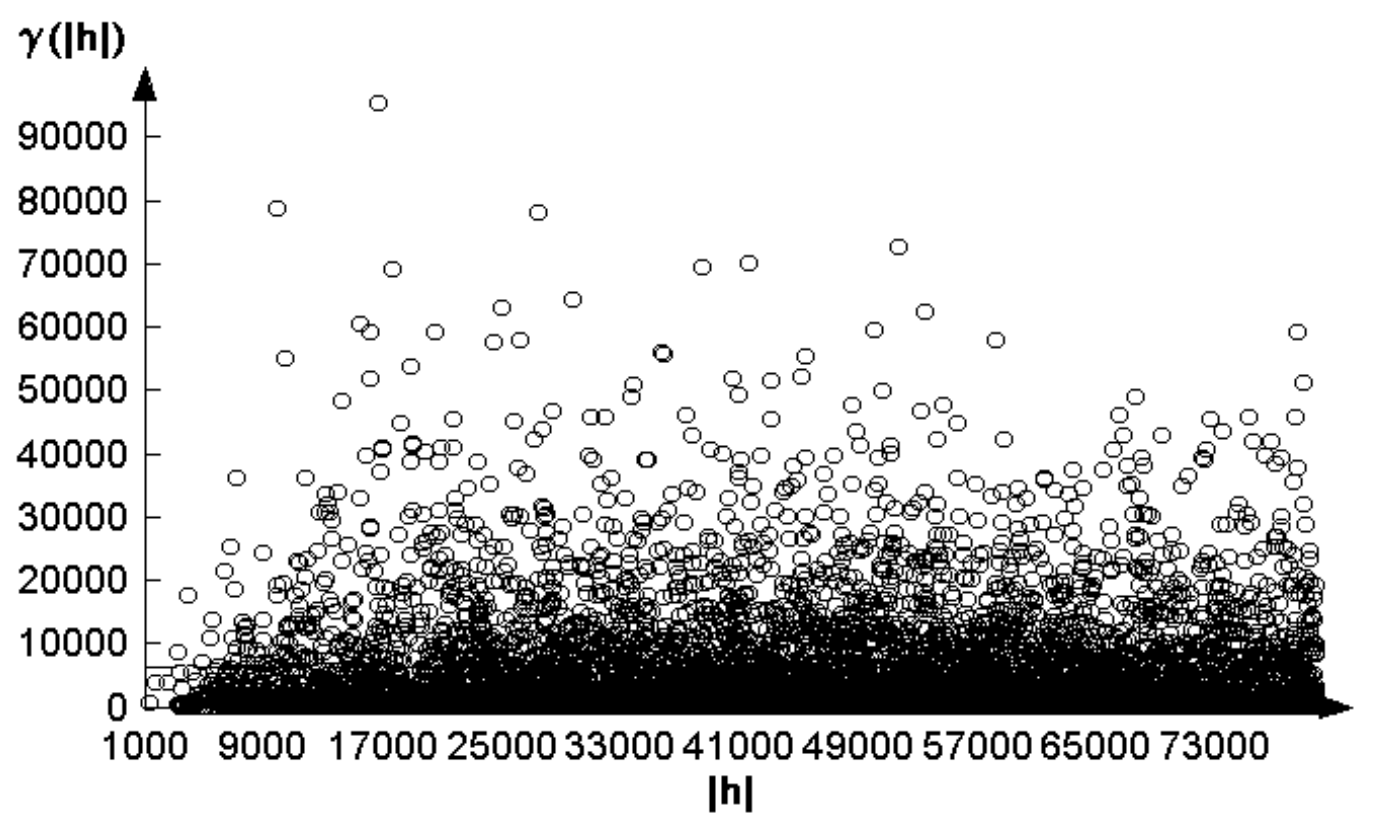

Besonders hohe Differenzen werden also bei geringem räumlichem Abstand nur im Zusammenhang mit der Lage im Harz beobachtet. Somit kann die wesentlich höhere Semivarianz empirischer Semivariogramme bei geringem räumlichem Abstand für das integrierte DWD/HWW-Datenkollektiv durch den hohen Anteil von Harzstationen erklärt werden. Die Verhältnisse im Harz sind durch die starke Reliefierung sehr heterogen. Eine ausschließliche Meßnetzverdichtung in diesem Gebiet durch die Erweiterung um die HWW-Daten muß folglich eine Erhöhung der Semivarianz für kleine $|\mathrm{h}|$ nach sich ziehen. Eine Ortsabhängigkeit der räumlichen Autokorrelation bzw. der Semivarianz widerspricht grundsätzlich der Voraussetzung der Stationarität. Diese Abweichung wurde jedoch nicht als so gravierend angesehen, daß eine weitere Teilung des Untersuchungsgebiets Bergland für notwendig erachtet wurde. Zudem wurde dadurch keine Verbesserung der Präzision erwartet, welche die größere Komplexität der Umsetzung in Form eines Gesamtmodells für Niedersachsen gerechtfertigt hätte. Gegen eine weitere Teilung in diesem Sinne spricht zudem, daß eine dann für eine flächige Modellumsetzung notwendige Definition einer Übergangszone zwischen Harz und restlichem niedersächsischen 
Bergland eine größere flächenmäßige Ausdehnung hätte als das neu auszuscheidende Untergebiet Harz. Hier wurde klar zu Gunsten der Einfachheit der Regionalisierung entschieden. Die von den Berglandmodellen erreichte Präzision - sowohl DWD- als auch DWD/HWW-Modelle - kann zudem bereits ohne anschließende geostatistische Analyse im Verhältnis zur Meßgenauigkeit (s. S. 74) als sehr gut angesehen werden.

Für Anwender, die sich ausschließlich für den niedersächsischen Harz interessieren wurden Modelle für dieses Untersuchungsgebiet in Anhang 11 dargestellt. Ihre Zusammensetzung entspricht weitestgehend derjenigen der HWW-Modelle. Sie werden im Rahmen dieser Arbeit nicht gesondert diskutiert.

Abschließend muß beurteilt werden, welche Modelle zur Regionalisierung der monatlichen und jährlichen Niederschlagssummen grundsätzlich angewendet werden sollten. Zur Auswahl stehen zwei Möglichkeiten. Die Verwendung der Modelle des reinen DWD-Datenkollektivs oder der Modelle des integrierten DWD/HWWDatenkollektivs. Eine ausschließlich DWD-gestützte Regionalisierung zeichnet sich durch eine sehr gute Anpassung der Modelle an die Daten (hohe Präzision) aus. Die Erweiterung um die HWW-Daten führt zu einer hohen Meßnetzdichte in einem - überdurchschnittlich heterogenen - Teilgebiet, die sich in einer schlechteren Modellierbarkeit der räumlichen Autokorrelation der Residuen widerspiegelt. Die Abweichungen von der angenommenen Stationarität bestehen andererseits auch für das reine DWD-Datenkollektiv, obwohl sie sich bei dessen Analyse nicht zeigen. Daher wurde im Verzicht auf Integration der HWW-Daten kein wirklicher Vorteil für die Regionalisierung gesehen. Die Verbesserung der Repräsentanz für das Untersuchungsgebiet (Kap.4.1.3.1) überwiegt die Probleme durch die partielle Meßnetzverdichtung. Die Modelle des integrierten DWD/HWW-Datenkollektivs werden daher favorisiert.

\subsubsection{Niederschlag im Tiefland}

Die Regionalisierung der durchschnittlichen monatlichen und jährlichen Niederschlagssummen im niedersächsischen Tiefland stützte sich auf Daten von 260 Stationen des Deutschen Wetterdienstes (Kap. 4.1.3.2). An eine schrittweise lineare Regression schloß sich jeweils eine iterative geostatistische Analyse an, wie sie in Kap 2.5 beschrieben wird. 
Die in Tabelle 55 auf Seite 126 dargestellten Modelle der durchschnittlichen monatlichen und jährlichen Niederschlagssummen im niedersächsischen Tiefland werden durch drei räumliche Variablen gebildet: Geländehöhe, Rechtswert und Abstand zur Küste. Angesichts der Tatsache, daß es sich bei dem Untersuchungsgebiet Tiefland um ein zum größten Teil nur schwach reliefiertes Gelände handelt, kann es nicht verwundern, daß die größte Bedeutung für die Varianzaufklärung der Zielvariablen hier nicht der Geländehöhe zukommt (s. Abbildung 30, S. 127). Statt dessen sind es die Variablen der geographischen Lage, die den größten Anteil am Bestimmtheitsmaß der Modelle haben.

Die Ergebnisse von FelbermeIER (1994), Klein (1994), SABOROwsKi \& STOCK (1994), BÖHNER (1996), STEINER \& LEXER (1998) oder auch VENT-SCHMIDT (1985) zeigten für ihre Untersuchungsgebiete einen Zusammenhang zwischen Niederschlag einerseits und Geländehöhe und geographischer Lage andererseits. Das Erklärungspotential der einzelnen Variablen ist dabei je nach Untersuchungsgebiet unterschiedlich, leider aber auch nicht immer dargestellt worden. Erkennbar ist, daß in Gebieten wie dem Harz (SABOROWSKI \& STOCK, 1994) die Bedeutung der Variable Geländehöhe deutlich überwiegt, während für weniger stark reliefierte Gebiete Variablen der geographischen Lage zumindest als bedeutend angesehen werden. Diese Einschätzung wird durch die für das Tiefland mit schrittweiser linearer Regression gebildeten Modelle bestätigt. Mit abnehmendem maritimem Einfluß, sprich bei größerem Abstand zur Küste oder höherem Rechtswert (Ausnahme: August), werden geringere durchschnittliche Niederschlagssummen geschätzt.

Insgesamt erreichen die Regressionsmodelle des Tieflands nicht die hohe Varianzaufklärung der Berglandmodelle. Die Bestimmtheitsmaße liegen für alle Monate außer Oktober $(81,4 \%)$ und November $(72,8 \%)$ - zum Teil deutlich - unter $70 \%$, für Februar $(36,0 \%)$ und April (30,4\%) sogar unter $40 \%$. Dennoch sind die Werte des RMSE geringer als im Fall der Berglandmodelle, was sich durch die insgesamt geringere Varianz der Meßwerte in diesem Teil des Untersuchungsgebietes erklären läßt (vgl. Standardabweichungen in Tabelle 28, S. 80). Bei geringer Varianz kann auch schon eine geringe Varianzaufklärung im Verhältnis zu der Meßungenauigkeit zu ausreichend genauen Modellen führen. Nehmen wir ausgehend von Tabelle 21 auf Seite 74 einen gering angesetzten Fehlerrahmen von $\pm 5 \%$ an, so entsprechen diese $5 \%$ für einen Mittelwert über alle 
Monate von ca. $60 \mathrm{~mm}$ einem Rahmen von $\pm 3 \mathrm{~mm}$. Bei Werten des RMSE der monatlichen Modelle von 3 bis $6,5 \mathrm{~mm}$ und der Möglichkeit, daß im niedersächsischen Tiefland die Meßfehler durch Windfelddeformationen um den Regenmesser erheblich über $5 \%$ liegen können, kann der Modellfehler als ausreichend gering angesehen werden. Es erscheint wenig sinnvoll, genauere und damit aufwendigere Modelle zu bilden. Lediglich die Definition und Verwendung neuer Variablen könnte auf dem Weg der Regression noch Verbesserungen bewirken, was jedoch als wenig aussichtsreich angesehen wird.

Statt einer genaueren Modellanpassung an die Meßdaten wurde daher eine Beschreibung der räumlichen Autokorrelation der Regressionsresiduen angestrebt. Durch die vorherige schrittweise lineare Regression kann angenommen werden, daß bedeutende räumliche Trends, welche die geostatistische Analyse stören würden, bereits aus den Daten eliminiert werden konnten. Daß dies der Fall ist, zeigen die als typisch zu bezeichnenden empirischen Semivariogramme (z. B. Abbildung 31, S. 128). Theoretische Semivariogramme in Form sphärischer Modelle können problemlos angepaßt werden (s. Tabelle 56).

Wie erfolgreich die Beschreibung der räumlichen Autokorrelation zur Verbesserung der Regression genutzt werden kann, zeigt die Kreuzvalidierung (s. Tabelle 57, S. 129). Mit einer absoluten Verbesserung um durchschnittlich $1 \mathrm{~mm}$ und einer relativen Verringerung des durchschnittlichen Schätzfehlers um $12 \%$ (April) bis $43 \%$ (September) ist der zur Modellierung notwendige Aufwand gerechtfertigt.

Zusammenfassend läßt sich zur Modellierung der durchschnittlichen monatlichen und jährlichen Niederschlagssummen im niedersächsischen Tiefland feststellen: Mit den drei räumlichen Variablen Rechtswert, Geländehöhe und Abstand zur Küste konnten plausible Regressionsmodelle gebildet werden, die für nahezu das gesamte niedersächsische Tiefland repräsentativ sein dürften (Kap.4.1.3.2). Der Fehler der OLS-Regressionen, als RMSE quantifiziert, liegt für die monatlichen Modelle stets unter $7 \mathrm{~mm}$, für das Modell der jährlichen Niederschlagssumme bei $41 \mathrm{~mm}$. Dies sind außer für den März (12\%) stets Werte in Höhe von $6 \%$ bis $9 \%$ des arithmetischen Mittels der Meßwerte (Tabelle 28, S. 80). Durch das Kriging wird der Fehler der Regionalisierung noch verringert, wie die Ergebnisse der Kreuzvalidierung (Tabelle 57 S. 129) zeigen. 
Der Regionalisierungsfehler muß nach den Ergebnissen im Rahmen der Meßgenauigkeit der Niederschlagsmessungen mit dem Hellmann-Regenmesser liegen. Diese wird von KumM (1983) mit bis zu $-20 \%$ im Sommer und $-27 \%$ im Winter (Schneeverwehungen) und von KURTYKA (1953; aus KLEIN, 1994) mit -5\% bis $-80 \%$ in Abhängigkeit von der Windexposition angegeben. Eine Variable wie die Exponiertheit zum Umgebungsminimum sollte diese Windexposition beschreiben können. Die Zusammenhänge in den Datenkollektiven waren jedoch nicht so stark, als daß diese Variable bei der Modellbildung berücksichtigt worden wäre. Eine räumliche Analyse der von KUMM (1983) verwendeten Fehler zwischen Regenmessungen mit und ohne Windschutz unter Verwendung dieser und/oder ähnlicher räumlicher Variablen könnte eine lohnende Aufgabe sein. Entstehende Modelle könnten zur Korrektur von Niederschlagsmessungen verwendet werden.

\subsubsection{Relative Luftfeuchte}

Das Datenkollektiv zur Regionalisierung der relativen Luftfeuchte besteht aus Daten von 44 Stationen des DWD, das sich vom Temperaturdatenkollektiv nur durch die Reduzierung um die Station „Einbeck“ unterscheidet (Kap. 4.1.4). Zunächst soll die Erhöhung der Präzision durch die Aufspaltung des Datenkollektivs in ein Berglandund ein Tieflandkollektiv diskutiert werden. In den Unterkapiteln folgt sodann die Diskussion der Teilkollektivmodelle.

Durch die Teilung der Daten in ein Bergland- und ein Tieflanddatenkollektiv konnte eine Erhöhung der Präzision nicht eindeutig festgestellt werden. Allerdings verwenden überlegene Gesamtmodelle stets mehr Regressoren als die entsprechenden Teilkollektivmodelle. Umgekehrt ist dies nicht der Fall (Tabelle 60, S. 131). Vor allem die Tieflanddaten werden durch die spezielleren Modelle zumeist besser beschrieben als durch die Gesamtmodelle. Die Tieflandmodelle für Oktober und November sind mit nur einem Regressor besser an die Tieflanddaten angepaßt als das entsprechende Gesamtmodell mit 3 Regressoren.

In der Betrachtung von Präzision und Einfachheit der Modelle sind somit die Modelle der Teilkollektive $\mathrm{zu}$ bevorzugen. Vor allem die homogeneren Tieflanddaten können auf diesem Wege genauer beschrieben werden als im Verbund mit den stärker streuenden Berglanddaten (s. Tabelle 31, S. 83 und Tabelle 32, 
S. 84). Der Mehraufwand durch die getrennte Modellierung fällt vor allem im Vergleich mit dem Aufwand zur Erstellung der Datenbasis nicht ins Gewicht (s.a. Kap. 6.1). Da auch die Plausibilität für eine getrennte Modellierung der beiden Untergebiete spricht, wurde diese favorisiert.

\subsubsection{Relative Luftfeuchte im Bergland}

Die Parametrisierung der Berglandmodelle für die Regionalisierung der relativen Luftfeuchte zeigt für jeden Monat ein hohes Erklärungspotential von höhenabhängigen Variablen (Geländehöhe und Lee-Effekt 50). Zudem können die lageabhängige Variable Rechtswert und der Anteil von Bebauung an der Landnutzung in einigen Modellen einen bedeutenden Teil der Varianz der Zielvariablen erklären (s. Tabelle 61, S. 132).

Der Einfluß der Geländehöhe auf die Lufttemperatur im Bergland wurde bereits beschrieben und diskutiert (Kap. 5.1.1.1 und Kap. 6.1.1.1). Wie in Kap.4.1.4 beschrieben wurde, ist die relative Luftfeuchte bei gleichem Wassergehalt der Luft im Falle geringer Lufttemperatur höher als bei hoher Lufttemperatur. Dieser Zusammenhang - große Geländehöhe, niedrige Lufttemperatur, hohe relative Luftfeuchte - kommt in allen Modellen mit Beteiligung der Geländehöhe (März bis August) durch positive Regressionskoeffizienten zum Ausdruck. Dieser Zusammenhang wird somit als plausibel eingestuft und führt $\mathrm{zu}$ beachtlichen Bestimmtheitsmaßen (s. Tabelle 61).

Die Variable Lee-Effekt 50 wird für die monatlichen Modelle von September bis Februar zur Regionalisierung der relativen Luftfeuchte mit negativen Regressionskoeffizienten herangezogen. Auch zur Regionalisierung der Niederschlagssummen (Juli bis September) fand sie mit negativen Regressionskoeffizienten Verwendung. Die räumliche Variable Lee-Effekt 25, zu deren Berechnung bei sonst gleicher Definition lediglich eine kleinere Umgebung betrachtet wird, findet in den Tieflandmodellen der Lufttemperatur für März, August und die durchschnittliche Jahrestemperatur Verwendung. In diesen Modellen zeigt die Variable Lee-Effekt 25 eine höhere Temperatur für solche Stationen an, welche im Windschatten (östlich) von Geländeerhebungen liegen. Sowohl die Modellierung höherer durchschnittlicher Lufttemperatur bei höheren Werten für Lee-Effekt 25 als auch geringere Niederschläge bei hohen Werten von Lee-Effekt 50 deuten auf die 
Plausibilität der beobachteten negativen Regressionskoeffizienten für Lee-Effekt 50 bei der Regionalisierung der relativen Luftfeuchte hin. Die Aufklärung der Varianz der Zielvariablen durch die räumliche Variable Lee-Effekt 50 scheint somit plausibel. Weshalb das Erklärungspotential dieser Variablen das der Geländehöhe in den Herbst- und Wintermonaten übersteigt, ist durch die vorhandene Datenlage nicht zu klären. Es kann vermutet werden, daß in den Frühlings- und Sommermonaten andere Windverhältnisse herrschen, in denen der Luftstrom aus westlicher Richtung keinen so großen Einfluß auf die Lufttemperatur und damit auf die relative Luftfeuchte hat.

Eine ungünstige Beeinflussung der Variablenermittlung und dadurch der Modellbildung durch die gewählte Berechnungsmethode von Lee-Effekt 50 (vgl. Kap. 3.3.3) wird nicht erwartet, kann aber nicht ausgeschlossen werden. Alternative Modelle ohne die Verwendung von Lee-Effekt 50 sind in Anhang 13 dargestellt, werden hier aber nicht weiter diskutiert. Die Bereiche, an denen die Variable LeeEffekt 50 nicht sicher bestimmt werden konnte, sind Abbildung $38 \mathrm{zu}$ entnehmen. Wie ebenfalls aus Abbildung 38 hervorgeht, sind von der Unsicherheit der Berechnung von Lee-Effekt 50 (Lee-Effekt 25) 15 (9) Luftfeuchtestationen im niedersächsischen Tiefland und 8 (4) Luftfeuchtestationen im niedersächsischen Bergland betroffen. Je näher eine Station an der westlichen oder auch südlichen oder nördlichen Grenze des Höhenmodells liegt, desto weniger sicher ist die Berechnung der Variable Lee-Effekt 50. Dies gilt entsprechend für Lee-Effekt 25. Da eine plausible Parametrisierung der Modelle mit Lee-Effekt 50 stattfand und bedeutende Fehler bei der Schätzung der Werte von Lee-Effekt 50 nicht erwartet werden, wurden die in Kapitel 5.1.3.1 vorgestellten Modelle favorisiert. 
Abbildung 38: Darstellung der Regionen und Stationen im niedersächsischen Bergland mit unvollständiger Bestimmung der DHM-Werte in einer 25 km- bzw. 50 km-Umgebung nach Westen

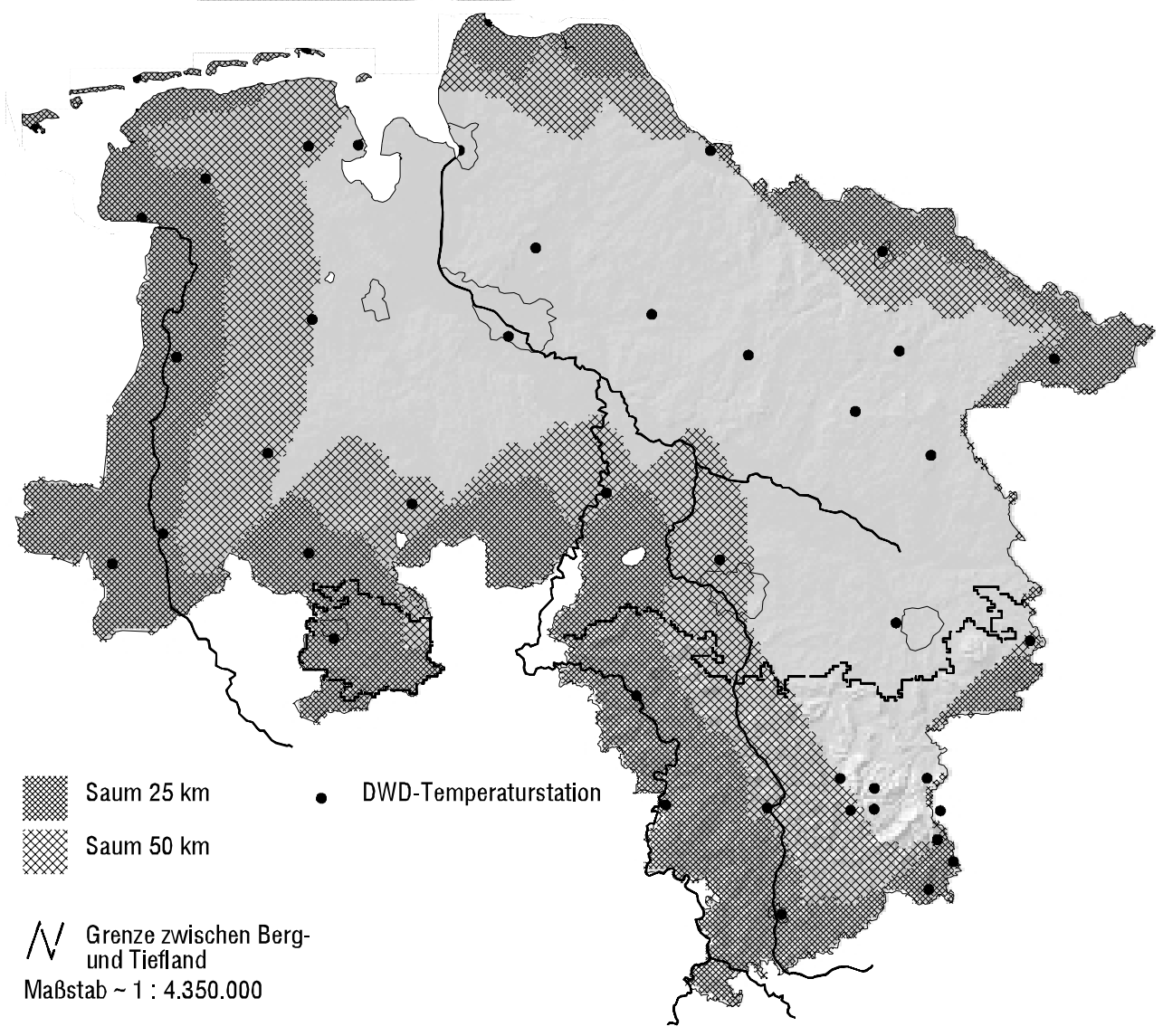

Die beiden Variablen Bebauung und Rechtswert sind bereits aus Temperaturmodellen des Tieflandes bekannt. Sie wurden wegen zu geringem Beitrag zum Bestimmtheitsmaß zwar nicht bei der Modellbildung zur Regionalisierung der Lufttemperatur im Bergland verwendet, zeigen aber einen plausiblen Zusammenhang mit der relativen Luftfeuchte. Je östlicher ein Standort, desto feuchter (weil kühler) ist die Luft (April bis August und Jahresdurchschnitt), je höher der Anteil der Bebauung an der Landnutzung, desto trockener (weil wärmer) ist die Luft (Februar, März, Mai bis August und Jahresdurchschnitt).

Die räumliche Variable QWKaltluftabflu $\beta$ wurde bei der Regionalisierung der relativen Luftfeuchte nicht zur Modellbildung herangezogen. Das eingeschränkte Erklärungspotential dieser Variable, die als bedeutend für die Höhe der Lufttemperatur ermittelt wurde (Kap. 5.1.1.1), kann auf die eingeschränkte 
Repräsentanz des Datenkollektivs bezüglich dieser Variable zurückgeführt werden. Die breiartige Viskosität der Kaltluft und die Meßsituation der DWD-Stationen überwiegend in Siedlungen könnten einen Einfluß von $Q$ WKaltluftabflu $\beta$ auf die Lufttemperatur und damit auch auf die relative Luftfeuchte verdecken.

In Tabelle 31 auf Seite 83 sind deskriptive Statistiken zu den Luftfeuchtemeßwerten der DWD-Stationen aufgeführt. Vergleicht man die Standardabweichungen der Meßwerte mit den in Tabelle 61 (S. 132) dargestellten Werten des RMSE, so fällt auf, daß die Standardabweichungen of nicht einmal doppelt so hoch sind wie die Werte des RMSE. Man kann daraus folgern, daß die Regionalisierung durch den Mittelwert der Meßwerte nur um das doppelte unsicherer ist als diejenige unter Verwendung der Regressionsgleichungen. Da die Regressionsgleichungen auf physikalisch und empirisch plausiblen Zusammenhängen beruhen, wird auf diesem Weg der Regionalisierung jedoch ein deutlich geringerer Regionalisierungsfehler erwartet.

Einschränkungen bezüglich der Repräsentanz des verwendeten Datenkollektivs (s. Kap. 4.1.4.1 und Kap. 4.1.2.1) sind selbstverständlich zu berücksichtigen. Bezüglich der räumlichen Variablen Geländehöhe, Lee-Effekt 50 und Rechtswert kann das Berglanddatenkollektiv zur Regionalisierung der relativen Luftfeuchte als weitestgehend repräsentativ angesehen werden. $\mathrm{Zu}$ ergänzen ist hier noch die Repräsentanzuntersuchung zur Variable Bebauung. Diese zeigt Werte von $0 \%$ bis $57,9 \%$ bei einem Mittelwert von 24,3\% und einer Standardabweichung von 16,1\%. Lediglich 0,3\% der Rasterwerte für Bebauung im niedersächsischen Bergland liegen über dem Maximum von 57,9\%, weshalb auch für diese Variable Repräsentanz angenommen wird. Die Einschränkungen bezüglich der Umsetzung der Modelle - Kappung der Variablenwerte zur Vermeidung von unplausiblen Modellwerten gelten jedoch wie auf Seite 66 oben beschrieben.

\subsubsection{Relative Luftfeuchte im Tiefland}

Die Stationen des zur Regionalisierung der relativen Luftfeuchte verwendeten Datenkollektivs entsprechen den DWD-Temperaturstationen des Tieflandes. Da eine enge Beziehung zwischen Lufttemperatur und relativer Luftfeuchte unterstellt werden kann (Kap. 4.1.4), ist $\mathrm{zu}$ erwarten, daß räumliche Variablen der Temperaturmodelle auch zur Varianzaufklärung der relativen Luftfeuchte beitragen 
können. Ein Vergleich der Regressionsmodelle (Tabelle 46, S. 112 und Tabelle 62, S. 134) bestätigt diese Erwartung. Nur die Geländehöhe kommt hinzu, die u.a. aus den Modellen zur Regionalisierung der durchschnittlichen Niederschlagssummen im niedersächsischen Tiefland bekannt ist. Die Variablen der geographischen Lage und die Landnutzungsvariable Gewässer werden allerdings nicht für die gleichen Monate zur Modellbildung herangezogen.

Die Präzision der Modelle, quantifiziert durch Bestimmtheitsmaß und RMSE in Tabelle 62, ist bis auf die Monate, in denen nur die Variable Bebauung einen signifikanten Beitrag zur Varianzaufklärung leisten konnte (September bis Februar), sehr hoch. Der größte Fehler (RMSE) wird allerdings für den Mai ermittelt (1,52\%), ist aber auch in diesem Fall nur ca. halb so groß wie die Standardabweichung der Meßwerte (vgl. Tabelle 32).

Die Landnutzungsvariable Bebauung wurde in allen Modellen verwendet. Die Regressionskoeffizienten sind für alle Monate negativ. Dies stimmt mit den in Kap. 6.1.1.2 gemachten Aussagen über die höhere Lufttemperatur bei hohem Anteil bebauter Flächen überein, die zur Senkung der relativen Luftfeuchte führen muß. Die Beträge des Regressionskoeffizienten für Bebauung zeigen ein Maximum im Mai. Dieser Monat zeichnet sich auch durch die höchsten Standardabweichungen der Meßdaten aus. Wegen der unterschiedlichen Modellzusammensetzungen sind die Regressionskoeffizienten der Monatsmodelle zwar nicht direkt vergleichbar, ein Zusammenhang zwischen den Standardabweichungen der Meßwerte und der Höhe der Regressionskoeffizienten kann aber dennoch erkannt werden.

Die positiven Regressionskoeffizienten der Variable Gewässer im Frühjahr (März bis Juni) kann durch zwei Zusammenhänge erklärt werden. Erstens steigt durch das Vorhandensein offener Wasserflächen die Verfügbarkeit von Wasser zur Evaporation und damit zur Steigerung des Wassergehaltes der Luft. Über offenen Wasserflächen entspricht die tatsächliche Evaporation der potentiellen, während sie über Boden oder bewachsenem Boden im allgemeinen darunter liegt (vgl. HÄCKEL, 1990, S. 64). Zweitens führen offene Gewässer im Frühjahr zu einer Abkühlung der Lufttemperatur durch die durchschnittlich kühleren Temperaturen des im Winter abgekühlten Wassers. Da sich in den anderen Monaten diese beiden Faktoren nicht gleichermaßen ergänzen, sondern sogar gegenläufig auf die Lufttemperatur auswirken können - im Herbst führen Gewässer zu einer langsameren Abkühlung 
der durchschnittlichen Lufttemperatur - ist auch das Fehlen der Variable Gewässer in den anderen Monaten erklärbar und plausibel.

Plausibel erscheinen ebenfalls die Regressionskoeffizienten der Variablen der geographischen Lage. Gerade in gemeinsamer Betrachtung mit der Landnutzungsvariable Gewässer kann folgende Beobachtung gemacht werden: Der Hochwert und der Abstand zur Küste gehen im gleichen Zeitraum in die Modelle ein wie Gewässer. Sie beschreiben zudem mit dem Abstand zur Küste und der geographischen Lage die gleichen Effekte, die oben für die Variable Gewässer beschrieben wurden. Bei großem Hochwert bzw. geringem Abstand zur Küste folgt nördlichere Lage (Temperatur $\downarrow$, rel. Luftfeuchte $\uparrow$ ) und von Ende des Winters bis zum Hochsommer durch die höhere Atlantizität der gleichgerichtete Temperatureffekt (Temperatur $\downarrow$, rel. Luftfeuchte $\uparrow$ ) und das Vorhandensein von höherem Wasserangebot. Vom Spätsommer bis zum Winter führt ein stärkerer atlantischer Einfluß zu höheren durchschnittlichen Lufttemperaturen (rel. Luftfeuchte $\downarrow)$, was den beiden anderen Effekten entgegensteht und somit erklärt, weshalb in diesem Zeitraum beide lageabhängigen Variablen nicht an der Modellbildung beteiligt sind.

Betrachtet man die Höhe der Beträge der Regressionskoeffizienten in Tabelle 62 - graphisch unten in Abbildung 39 dargestellt-, so bewirkt die Beteiligung der Variable Gewässer, daß der Beitrag der beiden anderen Variablen zur Varianzaufklärung der Zielvariablen geringer wird. Ansonsten läßt sich ein saisonaler Verlauf des Zusammenhanges auch für die beiden anderen Variablen erwarten, was durch die in Abbildung 40 dargestellten Korrelationskoeffizienten der drei Variablen mit den durchschnittlichen monatlichen Meßwerten der relativen Luftfeuchte bestätigt wird. Hier zeigt sich ganz eindeutig ein saisonaler Verlauf mit einem Maximum der Korrelation im Zeitraum von Februar/März bis August. 
Abbildung 39: Darstellung der standardisierten Beträge der Regressionskoeffizienten der drei räumlichen Variablen Abstand zur Küste, Hochwert und Gewässer in den Modellen zur Regionalisierung der relativen Luftfeuchte im niedersächsischen Tiefland

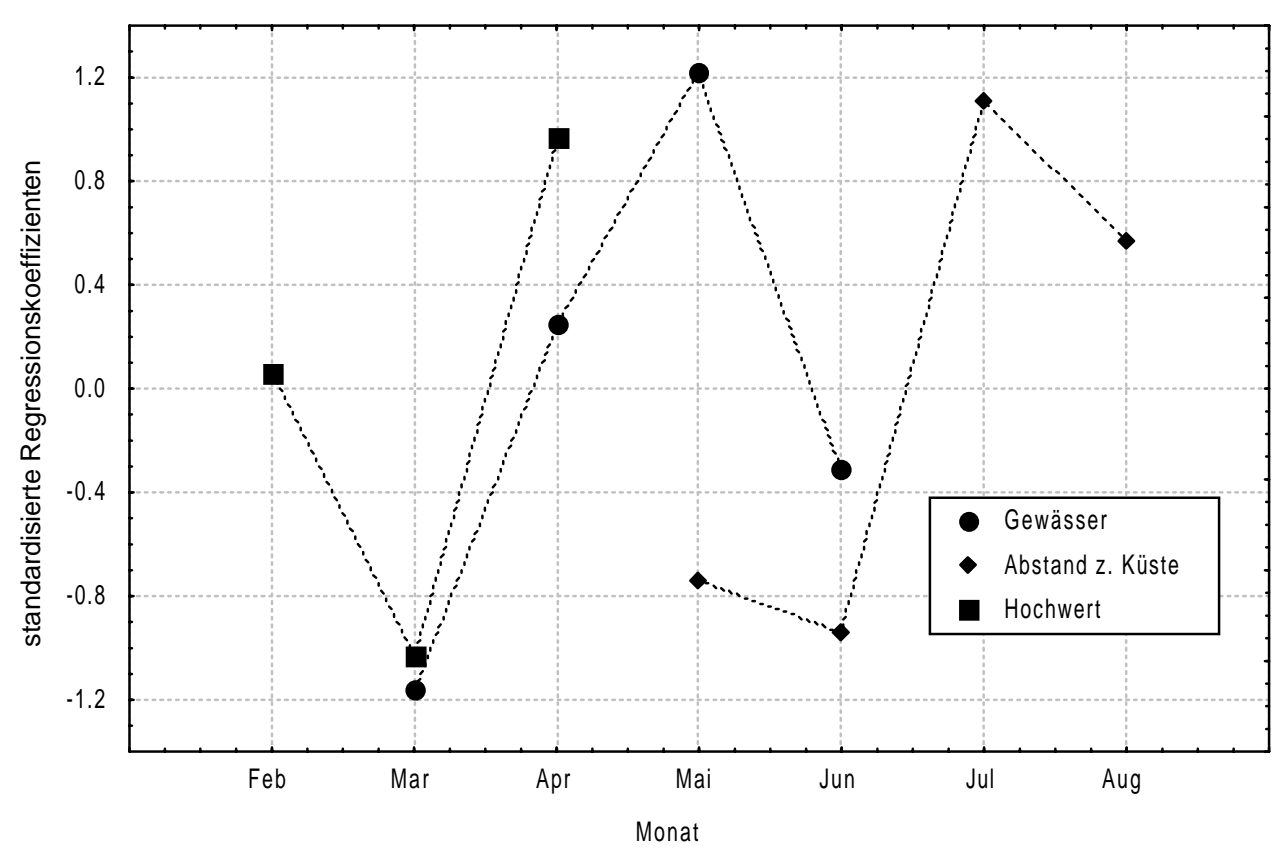

Abbildung 40: Darstellung der Korrelationskoeffizienten nach Pearson der drei räumlichen Variablen Abstand zur Küste, Hochwert und Gewässer mit den durchschnittlichen monatlichen Werten der relativen Luftfeuchte im niedersächsischen Tiefland

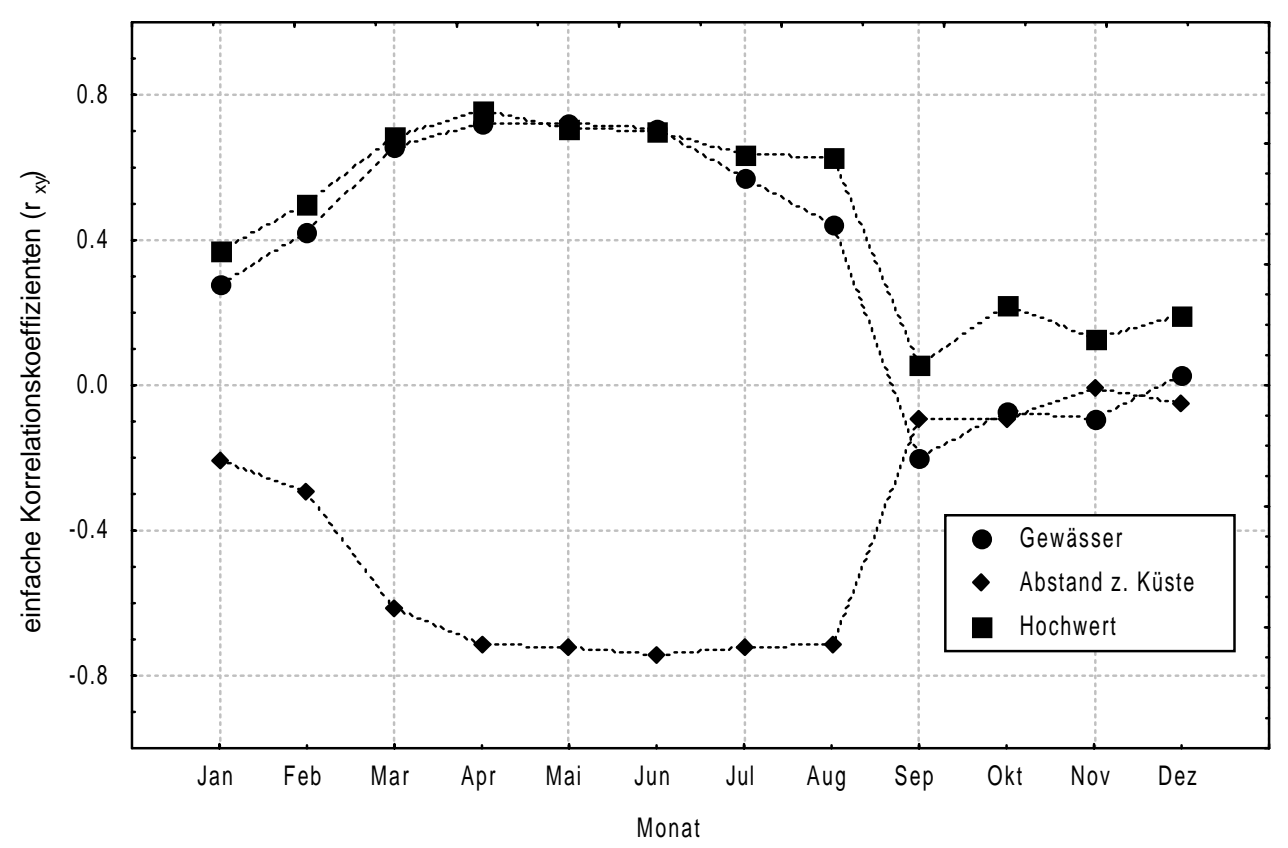

Plausibel scheinen auch die Werte für die höhenabhängigen Variablen Geländehöhe und Exponiertheit zum Umgebungsminimum. Bei höheren Werten dieser beiden 
Variablen wird eine niedrigere Lufttemperatur erwartet, die wiederum zu einer höheren relativen Luftfeuchte führt. Weshalb die beiden höhenabhängigen Variablen so ungleichmäßig Eingang in die Modelle finden und auch im Herbst und Winter keinen signifikanten Zusammenhang zur relativen Luftfeuchte zeigen, kann aufgrund der vorliegenden Daten nicht geklärt werden.

Während des größten Teils der Vegetationsperiode konnten somit plausible Modelle hoher Präzision für die räumliche Verteilung der relativen Luftfeuchte gefunden werden. $\mathrm{Zu}$ den in Kap.4.1.4.2 bzw. Kap. 4.1.2.2 gemachten Beobachtungen zur Repräsentanz der Meßsituation der Stationen kann hier auch die Repräsentanz für die Landnutzungsvariablen Gewässer und Bebauung bestätigt werden. Lediglich 0,4\% aller Rasterwerte liegen über dem Maximum der Bebauungswerte des Datenkollektivs, 0,3 \% über dem Maximum der Gewässerwerte. Zur Umsetzung der Modelle (s. Kap. Fehler! Verweisquelle konnte nicht gefunden werden.) wurde eine Kappung der Rasterwerte beim Maximum der Stationswerte vorgenommen.

\subsection{Depositionen}

Die Diskussion der in Kap. 5.2 dargestellten Regionalisierungsmodelle für die Freilanddepositionen und die von diesen abhängigen Anreicherungsfaktoren für die Bestandesniederschläge konzentriert sich auf drei voneinander abhängige Fragestellungen:

- Sind die modellierten Zusammenhänge (Regressionskoeffizienten) plausibel?

- Liegen die Modellwerte der gefundenen Regionalisierungsmodelle in einem plausiblen Wertebereich?

- Sind die Modelle für eine praxisrelevante Aussage zuverlässig bzw. repräsentativ genug? 


\subsubsection{Ausschließliche Betrachtung der Freilanddepositionen}

Entsprechend der in Kap. 2.5 und Kap. 4.2.4 dargestellten Methodik war der erste Schritt der Depositionsregionalisierung eine schrittweise lineare Regression. Zielvariablen waren die mittleren Einträge (1986 bis 1990) von Sulfat-Schwefel, Nitrat-Stickstoff, Ammonium-Stickstoff, Chlor und Protonen sowie der mittlere $\mathrm{pH}$ Wert. Die ermittelten Regressionsmodelle sind in Tabelle 63 auf Seite 136 aufgeführt, die zugehörigen Statistiken zur Präzision $\left(\mathrm{R}^{2}, \mathrm{RMSE}\right.$ und C.V.) in Tabelle 64 zusammengefaßt.

Die Modellfehler (RMSE) der Regressionsmodelle für Sulfat-Schwefel, NitratStickstoff und Ammonium-Stickstoff zeigen vor allem in Relation $\mathrm{zu}$ den Mittelwerten der abhängigen Variablen (Variationskoeffizient C.V) eine gute Anpassung an die Daten. Die linearen Modelle für den Chlor- und den Protoneneintrag weisen größere Modellfehler auf. Dies gilt im Fall des Chlormodells trotz des höchsten Bestimmtheitsmaßes aller angepaßten Freilandmodelle. Dieser Gegensatz - hohes Bestimmtheitsmaß und großer mittlerer quadratischer Fehler des Regressionsmodells - läßt sich nur über eine Besonderheit des Chlordatenkollektivs erklären (vgl. Kap. 4.2.2). Durch drei Meßstationen mit sehr hohen Chlor-Einträgen in Küstennähe ist die Gesamtvarianz deutlich höher als ohne diese drei Stationen. Dadurch wird - durch die räumliche Variable Gewässer- ein großer Teil der Zielvariablenvarianz erklärt, während der Modellfehler im Verhältnis zum Mittelwert, der durch die vielen Meßstationen mit geringen Einträgen geprägt ist, hohe Werte zeigt (Abbildung 41).

Abbildung 41 stellt das Regressionsmodell für die Chlor-Freilanddepositionen dreidimensional dar. Es verdeutlicht die positive Korrelation mit Gewässer und die negative mit Abstand zur Küste. 
Abbildung 41: Regionalisierungsmodell für den mittleren Chloreintrag von 1986 bis 1990 und punkthafte Darstellung der zugrundeliegenden Meßergebnisse mit Regressionsresiduen

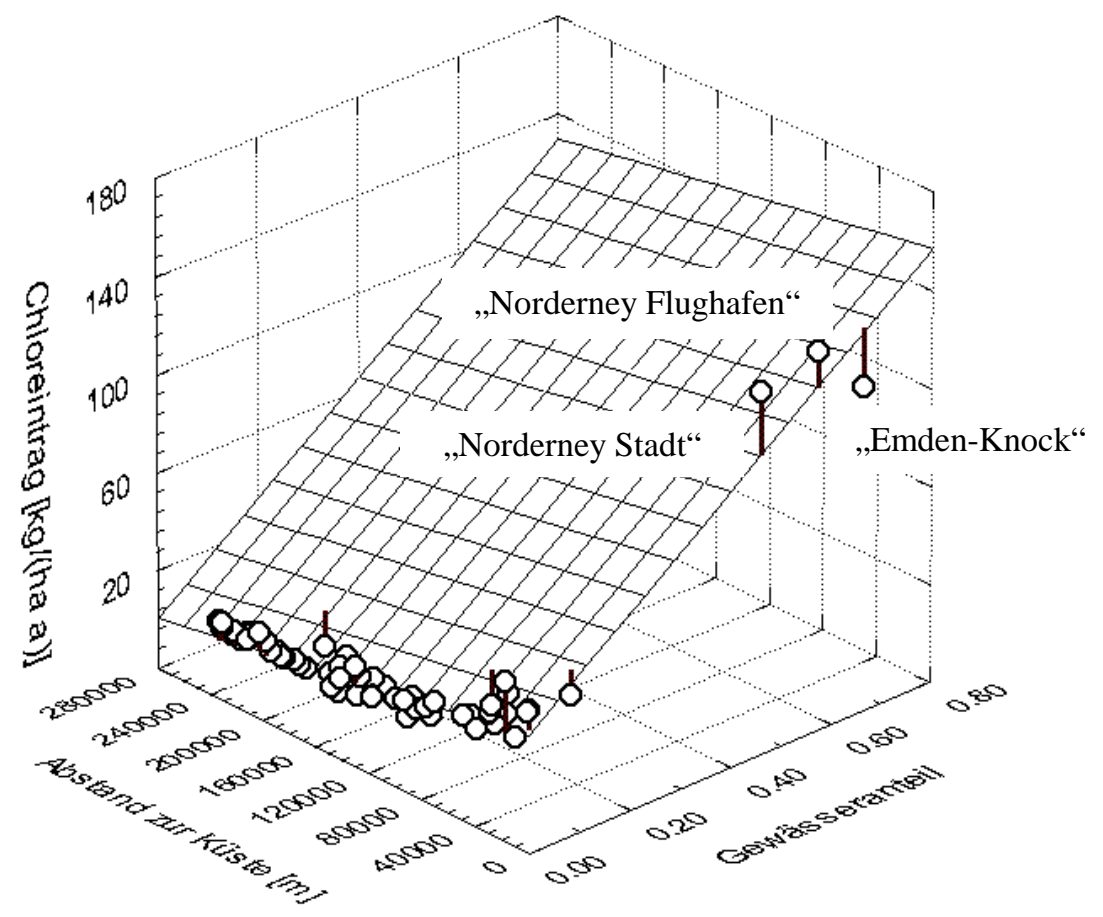

Chlor ist ein meeresbürtiges Element, weshalb der Abstand zur Küste als Einflußfaktor für die Höhe des Chloreintrages gilt (z. B. BREDEMEIER, 1987). BUSCH (1994) konnte durch ihre Modellierung der nassen Deposition so z. B. nachweisen, daß bei lang anhaltenden Regenfällen eine schnelle Abnahme der Chlorkonzentration im Niederschlag auftrat, woraus sie folgerte, daß es am beobachteten inländischen Meßort „keinen Nachschub aus lokalen Quellen“ gibt. Zudem zeigte das zugrunde liegende Datenmaterial eine Abnahme der Chlor-Deposition „mit zunehmender Entfernung vom Meer etwa von Nordnordwest nach Südsüdost“.

Ein lineares Regressionsmodell, das sich nur auf die räumliche Variable Abstand zur Küste stützt, kann die drei höchsten gemessenen Zielvariablenwerte nicht annähernd genauso gut beschreiben, wie das hier dargestellte bilineare Modelle, ohne für die niedrigen Meßwerte bei ähnlich geringem Abstand zur Küste sehr hohe Modellfehler in Kauf zu nehmen. Die Hinzunahme von Gewässer ermöglicht die Beschreibung eines Unterschieds zwischen den drei extrem hohen Meßwerten und allen anderen. Es fehlen zwar Meßwerte im mittleren Wertebereich von Gewässer, wodurch eine Bestätigung des hier linear modellierten Zusammenhanges nicht möglich ist. Durch die Daten besteht aber auch kein Widerspruch zur angenommenen Linearität. 
Nicht nur das Vorzeichen, auch die Höhe der Regressionskoeffizienten scheint plausibel. So führt eine Umgebung von $100 \%$ Gewässer zu einer Erhöhung des Modellwertes für den Chloreintrag um 161,43 kg (ha•a) ${ }^{-1}$. Bei einem Abstand zur Küste von $0 \mathrm{~m}$ ist somit ein maximaler Chloreintrag von $189,32 \mathrm{~kg}$ (ha•a) ${ }^{-1}$ möglich (maximaler Meßwert: 133,5 kg (ha・a) ${ }^{-1}$ ). Dieser theoretisch mögliche Eintrag wird auch durch die Implementierung des Modells im GIS erreicht. Das theoretisch erreichbare - unplausibel negative - Minimum von $-1,4 \mathrm{~kg}(\mathrm{ha} \bullet)^{-1} \quad(27,89$ 0,000097*302000) wird dagegen im GIS nicht realisiert. Die entsprechenden extremen Werte der beiden betroffenen Variablen koinzidieren an keinem Rasterpunkt im Bereich Niedersachsens. Das im GIS realisierte Minimum beträgt $3,3 \mathrm{~kg}(\mathrm{ha} \cdot \mathrm{a})^{-1}$.

Sowohl die Regressionskoeffizienten als auch die Modellwerte der gefundenen Modelle für den Chloreintrag im Freiland können als plausibel angesehen werden. Da das Erklärungspotential von Gewässer für den Chloreintrag vermutlich eher auf dem Meeresanteil in der Umgebung beruht als auf dem von Binnengewässern, wurde auch eine entsprechende Landnutzungsvariable Meer generiert und eine entsprechende Regression gerechnet:

$$
\text { frei }_{\mathrm{Cl}}=30.04+160.80 \cdot \text { Meer }-0.000103 \cdot \text { distkus }
$$

Die Regressionskoeffizienten weichen nur sehr schwach von denjenigen des Modells mit Gewässer ab. Im Binnenland führt das Modell den Chloreintrag nur auf eine räumliche Variable, den Abstand zur Küste (distkus) zurück. Nur in einem $2 \mathrm{~km}$ breiten Streifen hat Meer Einfluß auf das Modellergebnis. Daher wird auf eine Darstellung des Meer-Modells in Kapitel 10 verzichtet, obwohl es etwas plausibler erscheint als das Gewässer-Modell. Eine Optimierung der räumlichen Variablen war nicht Thema dieser Arbeit, wäre in diesem Fall aber sicherlich lohnend.

Auch bei der Regionalisierung des Ammoniumeintrages im Freiland wurde die Landnutzungsvariable Gewässer zur Modellbildung mit negativem Regressionskoeffizienten herangezogen. Dieser Einfluß ist erneut auf die drei Stationen mit den höchsten Werten für Gewässer zurückzuführen („Norderney Stadt“, „Norderney Flughafen“ und „Emden-Knock“). An diesen Stationen wurden im Verhältnis zu ihrer westlichen Lage und ihrer hohen durchschnittlichen jährlichen Niederschlagssumme recht niedrige Ammonium-Stickstoffeinträge gemessen. Eine solche Konstellation impliziert die Gefahr einer Scheinkorrelation zwischen der 
Zielvariablen und der räumlichen Variablen. Es erscheint jedoch aus zwei Gründen plausibel, daß bei hohen Werten von Gewässer auch geringere AmmoniumStickstoffeinträge zu erwarten sind.

Erstens ist in Gebieten mit hohem Anteil von Gewässer an der Landnutzung auch der Anteil von intensiver Landwirtschaft geringer. Da vor allem die Intensivviehhaltung als größter Emittent für Ammoniak gilt (z. B. ISERMANN \& ISERMANN, 1999 oder MEESENBURG et al., 1994), ist eine negative Korrelation zwischen Gewässer und dem Ammoniumeintrag daher $\mathrm{zu}$ erwarten. Betrachtet man nur diesen Wirkungszusammenhang, sollte aber die Landnutzungsvariable Acker (s. Kap. 3.4.1) wegen des direkteren Wirkungszusammenhanges ein größeres Erklärungspotential als Gewässer haben. Dies betont die Wirksamkeit des anderen hier eventuell auftretenden Effektes:

Zweitens liegt der Ammonium-Stickstoff in der Luft zunächst gasförmig in Form von Ammoniak vor. Gerade feuchte Oberflächen zeigen geringere Widerstände für gasförmige Depositionen (BURKHARD, 1994 oder GRAF \& SCHMID, 1989). Nicht nur für die Gewässer selbst sondern durch verstärkte Taubildung auch für Flächen in unmittelbarer Nähe von Gewässern sind daher verstärkte gasförmige Depositionen zu erwarten, welche zu einer Verringerung der Konzentration von Ammoniak in der Luft führt. Eine Erfassung der gasförmigen Immissionen unter Taubedingungen durch Bulk-Sammler erscheint in vollem Umfang unwahrscheinlich (an feuchten Oberflächen innerhalb des Sammlers weniger Luftbewegung als außerhalb). Wegen der Senkenfunktion von Gewässern für Ammoniak und der höheren Nebel- und Tauhäufigkeit in Gewässernähe kann daher grundsätzlich eine negative Korrelation zwischen Gewässer und Bulk-Depositionen, die nicht meeresbürtig sind, angenommen werden.

Bei einer Umgebung, die zu $100 \%$ aus Gewässern besteht, wird dem Regressionsmodell entsprechend $6,01 \mathrm{~kg}(\mathrm{ha} \bullet \mathrm{a})^{-1}$ weniger Ammonium-Stickstoff im Freiland deponiert als an einer Stelle ohne jegliches Gewässer im Umkreis von 2 km. Ca. $74 \%$ aller Rasterpunkte in Niedersachsen weisen den Wert 0 für Gewässer auf, ca. $95 \%$ liegen unter $12 \%$ Gewässer und $99 \%$ unter $40 \%$ Gewässer in einer 2 kmUmgebung. Der real auftretende Wertebereich des Beitrages durch die Landnutzungsvariable Gewässer beschränkt sich somit für ca. $99 \%$ aller Rasterpunkte in Niedersachsen auf den Bereich von 0 bis ca. $-2,4 \mathrm{~kg}(\text { ha } \bullet)^{-1}$. 
Größeren Einfluß auf das Modellergebnis für den Eintrag von Ammonium-Stickstoff haben die beiden anderen Regressoren, der Rechtswert und der durchschnittliche Jahresniederschlag. Ein Ort, der $100 \mathrm{~km}$ östlich von einem vergleichbaren gelegen ist, wird ein um $1,7 \mathrm{~kg}$ (ha•a) ${ }^{-1}$ reduzierter Eintrag geschätzt (Regressionskoeffizient -0,000017, s. Tabelle 63, S. 136). Einflußreicher noch als der Rechtswert ist die Variable Jahresniederschlag mit einem Regressionskoeffizienten von 0,00056. Schon der minimal für Niedersachsen regionalisierte durchschnittliche Jahresniederschlag von $53211 / 10 \mathrm{~mm}$ führt so $\mathrm{zu}$ einem Beitrag zum Modellergebnis von ca. 2,98 kg (ha•a) ${ }^{-1}$. Das Maximum von $157231 / 10 \mathrm{~mm}$ wird mit einem Beitrag von ca. 8,80 kg (ha•a) ${ }^{-1}$ Ammonium-Stickstoff modelliert.

Daß der Jahresniederschlag mit einem positiven Regressionskoeffizienten in das Regionalisierungsmodell eingeht, erscheint plausibel, da ein gasförmiger Stoff bei höheren Niederschlagssummen auch mit höherer Intensität ausgewaschen werden kann als an Orten geringer durchschnittlicher Niederschläge. Auch wenn die Dauer und Intensität der Niederschlagsereignisse bei der Variable Jahresniederschlag keine Berücksichtigung findet, erscheinen höhere Ammonium-Stickstoffeinträge bei höherem Jahresniederschlag plausibel.

Der negative Beitrag des Rechtswerts zum Regressionsmodell für den AmmoniumStickstoffeintrag ist vor dem Hintergrund einer im Westteil Niedersachsens und in den westlich gelegenen Niederlanden in großem Umfang betriebenen Intensivviehhaltung plausibel. $\mathrm{Ob}$ dieser Umstand sich linear in einem Regressionsmodell niederschlagen sollte, kann angezweifelt werden, die Daten ließen aber nicht auf eine bestimmte Transformation der Variable schließen. Wünschenswert wäre sicher die Verwendung einer räumlichen Variable zur Beschreibung der Viehdichte oder über die durchschnittliche Konzentration von Ammoniak in der Luft für das Zielgebiet.

Der Übersicht halber sind die theoretisch möglichen und die im GIS realisierten Minima und Maxima der Modellwerte in Tabelle 69 zusammengestellt. So wird deutlich, daß neben dem Chlormodell auch das Modell für den Eintrag von Ammonium-Stickstoff im Freiland theoretisch - bei Einsetzen der entsprechenden Extrema der räumlichen Variablen - negative Werte ermittelt werden können. Es zeigt sich jedoch durch den Einsatz des GIS, daß es im Bereich Niedersachsens keine Realisierung unplausibel negativer Werte gibt. Auch der im GIS realisierte maximale 
Modellwert von $11,8 \mathrm{~kg}(\mathrm{ha} \bullet)^{-1}$ Ammonium-Stickstoff liegt deutlich unter dem theoretisch möglichen Wert von $16,1 \mathrm{~kg}$ (ha•a) ${ }^{-1}$.

Tabelle 69: Theoretisch mögliche und im GIS realisierte Minima und Maxima der Modelle für die Freilanddepositionen

\begin{tabular}{lcccc}
\hline & \multicolumn{2}{c}{ theoretisch möglich } & \multicolumn{2}{c}{ im GIS realisiert } \\
& Min. & Max. & Min. & Max. \\
\hline Sulfat-Schwefel & 8,1 & 36,1 & 8,2 & 24,1 \\
Nitrat-Stickstoff & 5,2 & 10,6 & 5,2 & 10,5 \\
Ammonium-Stickstoff & $\mathbf{- 1 , 5}$ & 16,1 & 1,9 & 11,8 \\
Chlor & $\mathbf{- 1 , 4}$ & 189,3 & 3,3 & 189,3 \\
$\mathbf{H}^{+}$ & 0,1 & 0,6 & 0,1 & 0,6 \\
\hline pH & 4,2 & 5,4 & 4,2 & 5,4 \\
\hline
\end{tabular}

Die im Ammoniummodell verwendeten Variablen Jahresniederschlag, Gewässer und Rechtswert können als repräsentativ für das Zielgebiet Niedersachsen angesehen werden. Die verwendeten Stationen umfassen einen Bereich von 592 bis $1424 \mathrm{~mm}$ Niederschlag im Jahr und decken damit fast den gesamten Wertebereich für den Raum Niedersachsen (532 bis 1572 mm) ab. Die Verteilung der Variable Gewässer läßt zwar noch Wünsche offen (s.o. zum Chloreintrag), kann aber ebenso wie die gleichmäßige Verteilung der Rechtswerte als durchaus repräsentativ angesehen werden. Nur 0,2 \% liegen über dem Maximum für Gewässer von 66,5\%.

Der theoretisch mögliche Modellwert für den Nitrat-Stickstoffeintrag von $10,6 \mathrm{~kg}(\mathrm{ha} \bullet)^{-1}$ wird im GIS nur knapp verfehlt (Tabelle 69). Die im GIS realisierten Regionalisierungsergebnisse sind als plausibel einzustufen. Es fällt auf, daß keine Werte über $22 \mathrm{~kg}$ (ha•a) ${ }^{-1}$ Stickstoffeintrag im GIS erreicht werden. Tatsächlich werden Werte von 7,4 bis $21,9 \mathrm{~kg}(\mathrm{ha} \bullet \mathrm{a})^{-1}$ Gesamtstickstoffdeposition für das Freiland berechnet. Die Meßwerte lagen zwischen 9,2 und 21,7 kg (ha•a) ${ }^{-1}$. Dies und ähnlich hohe Angaben in FÜHRER et al. (1988) läßt die Modellwerte als durchaus plausibel erscheinen.

Die einzige räumliche Variable, die einen bedeutenden Teil der Varianz der Nitrateinträge aufklären konnte, war die Geländehöhe mit einem Regressionskoeffizienten von 0,00568 (s. Tabelle 63, S. 136). Nach den in Kap. 4.2.3 gemachten Aussagen kann das Nitratdatenkollektiv als absolut repräsentativ bezüglich dieser räumlichen Variable angesehen werden. Eine Geländehöhe von $1000 \mathrm{~m}$ führt mit der additiven Konstante von $5,22 \mathrm{~kg}(\mathrm{ha} \bullet \mathrm{a})^{-1}$ somit $\mathrm{zu}$ einem Modellwert von $10,90 \mathrm{~kg}(\mathrm{ha} \bullet \mathrm{a})^{-1} \quad$ Nitrat-Stickstoff. Daß nicht etwa der durchschnittliche Jahresniederschlag ein höheres Erklärungspotential für den Nitrat-Stickstoffeintrag 
besitzt, kann als Hinweis auf das Vorhandensein anderer Faktoren als der Niederschlagshöhe, die mit steigender Geländehöhe im Untersuchungsgebiet zu höheren Nitrat-Depositionen führen, interpretiert werden.

Die Variable Jahresniederschlag ist zudem das Ergebnis einer Regionalisierung von Meßergebnissen aus den Jahren 1961 bis 1990 (Kap. 5.1.2) und damit auf einen längeren Zeitraum bezogen als die Depositionsmessungen (1986 bis 1990). Dieser Unterschied wird allerdings als relativ unbedeutend angesehen, da sich die räumliche Verteilung der durchschnittlichen Jahresniederschläge von 1961 bis 1990 nicht wesentlich von der für die Jahre von 1986 bis 1990 unterscheiden sollte. An Orten mit relativ hohem (niedrigem) Niederschlag in der einen Periode kann mit hoher Wahrscheinlichkeit auch in der anderen Periode ein relativ hoher (niedriger) Niederschlag erwartet werden.

Welche anderen Faktoren die Höhe der Nitrat-Stickstoffeinträge beeinflußt haben, kann im Rahmen dieser Arbeit nicht geklärt werden. Eine größere Nebelhäufigkeit bei höherer Lage $\left(r_{\text {Pearson }}=0,48\right.$ bei 23 DWD-Stationen zur Nebelhäufigkeit; im Rahmen dieser Arbeit nicht beschrieben) ist dabei eine wahrscheinliche Ursache. Auch eine höhere Nitratkonzentration in der Luft über dem südniedersächsischen Bergland im Vergleich zum Tiefland könnte zu stärkerer Nitrat-Deposition führen, solche Überlegungen sind aber bei der vorliegenden Datenbasis nicht überprüfbar.

Die Varianz des Eintrages an Sulfat-Schwefel sollte grundsätzlich durch ähnliche Regressoren aufgeklärt werden können wie im Fall des Nitrat-Stickstoffs, da beide Stoffe in der Atmosphäre zunächst gasförmig vorliegen, durch ihre hohe Wasserlöslichkeit aber verstärkt durch Auswaschung Prozessen der Nassen Deposition unterliegen (Kap.4.2.1). Während aber die Nitrat-Emissionen in Deutschland vorwiegend auf den Kraftverkehr zurückzuführen sind, sind im Fall des Sulfats Industrie und private Feuerungsanlagen die größten Emittenten. Es ist daher nicht verwunderlich, daß im Rahmen der schrittweisen Regressionsanalyse noch vor der Geländehöhe (positiver Regressionskoeffizient) die Landnutzungsvariable Bebauung Eingang in das Regressionsmodell des Sulfat-Schwefeleintrages gefunden hat. Während für Gebiete mit höherer Siedlungsdichte höhere Einträge an SulfatSchwefel plausibel sind - BUSCH (1994) vermutet „lokale und regionale Nachschubquellen“-, wird für Wald eine verstärkte Filterwirkung durch Interzeptionsdeposition bezüglich der Luftschadstoffe beschrieben (z. B. MATZNER, 
1988). Es erscheint daher plausibel, daß auch die Landnutzungsvariable Wald mit einem hohen Erklärungspotential ( $\mathrm{R}^{2}$-Zunahme von knapp $\left.14 \%\right)$ und negativem Regressionskoeffizienten in das Sulfatmodell integriert werden konnte.

Bezüglich der Geländehöhe kann gemäß den in Kap. 4.2.3 gemachten Aussagen für das Sulfatdatenkollektiv volle Repräsentanz unterstellt werden. Was die beiden Landnutzungsvariablen Bebauung und Wald angeht, verschafft eine zweidimensionale Betrachtung der beiden Verteilungen einen guten Überblick.

Abbildung 42: Verteilungen von Bebauung und Wald des Sulfat-Schwefeldatenkollektivs

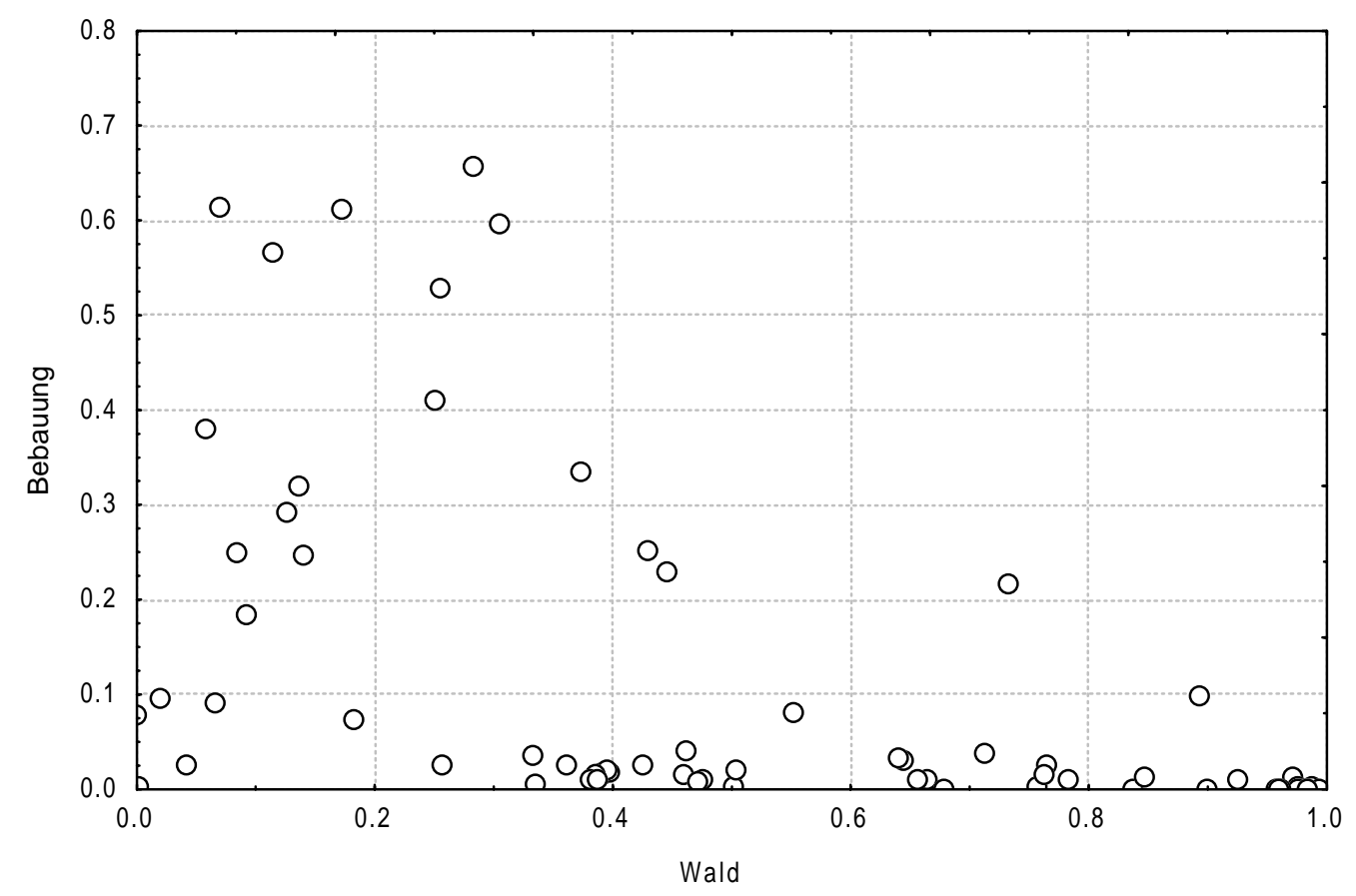

Insgesamt ist die Verteilung der Landnutzungsvariablen Wald gleichmäßiger und umfaßt den kompletten möglichen Wertebereich von $0 \%$ bis $100 \%$. Eine eingeschränkte Repräsentanz zeigt die Variable Bebauung. Allerdings liegen nur $0,2 \%$ aller Rasterzellen in Niedersachsen über dem Maximum des Sulfatdatenkollektivs von $66 \%$. Daher wird auch diese Variable als grundsätzlich repräsentativ für das Zielgebiet eingestuft. Bei eventuellen Extrapolationen über den im Datenkollektiv beprobten Wertebereich hinaus ist aber Vorsicht geboten. Die zur Kappung bei der Umsetzung von Modellen auf Seite 66 oben gemachten Aussagen wurden auch bei der Darstellung des Sulfatmodells in Kapitel Fehler! Verweisquelle konnte nicht gefunden werden. berücksichtigt. 
Der Wertebereich des Regionalisierungsmodells wird durch die Regressionskoeffizienten bestimmt. Diejenigen für welche die Landnutzungsvariablen Wald und Bebauung sind dabei recht einfach $\mathrm{zu}$ interpretieren. Ein Punkt mit $100 \%$ Bebauungsdichte (Wald) in der Umgebung hatte entsprechend des Regressionskoeffizienten von 8,60 (-5,73) zwischen 1986 und 1990 durchschnittlich um 8,60 (5,73) kg (ha•a) ${ }^{-1}$ höhere (niedrigere) jährliche Einträge an Sulfat-Schwefel (s. Tabelle 63, S. 136). Der Beitrag der Variable Bebauung ist allerdings wegen der eingeschränkten Repräsentanz bis $66 \%$ (s.o.) nur bis ca. 5,7 kg (ha•a) ${ }^{-1}$ abgesichert.

Der Regressionskoeffizient für die Geländehöhe von 0,0142 bedeutet eine Zunahme von $1,42 \mathrm{~kg}(\text { ha } \bullet)^{-1}$ durchschnittlichem Sulfat-Schwefeleintrag pro Anstieg der Geländehöhe um 100 m. Insgesamt läßt sich der Beitrag dieser Variable also mit 0 bis $13,5 \mathrm{~kg}(\mathrm{ha} \bullet \mathrm{a})^{-1}$ quantifizieren und ist somit der einflußreichste auf das Modellergebnis.

Die Modellresiduen deuten auf Abweichungen von der angenommenen Zufälligkeit der Residuen hin. Diese können größtenteils durch eine Abweichung der Beziehung zwischen dem Eintrag an Sulfat-Schwefel und der Landnutzungsvariablen Bebauung von der unterstellten Linearität erklärt werden (s. Abbildung 43). Die SulfatSchwefeleinträge der Stationen über 50 \% Siedlungsanteil in der Stationsumgebung deuten auf einen abnehmenden Trend bei sehr hoher Bebauungsdichte hin. 
Abbildung 43: Beziehung zwischen Bebauung und durchschnittlichem SulfatSchwefeleintrag mit linearer Regressionsgerade

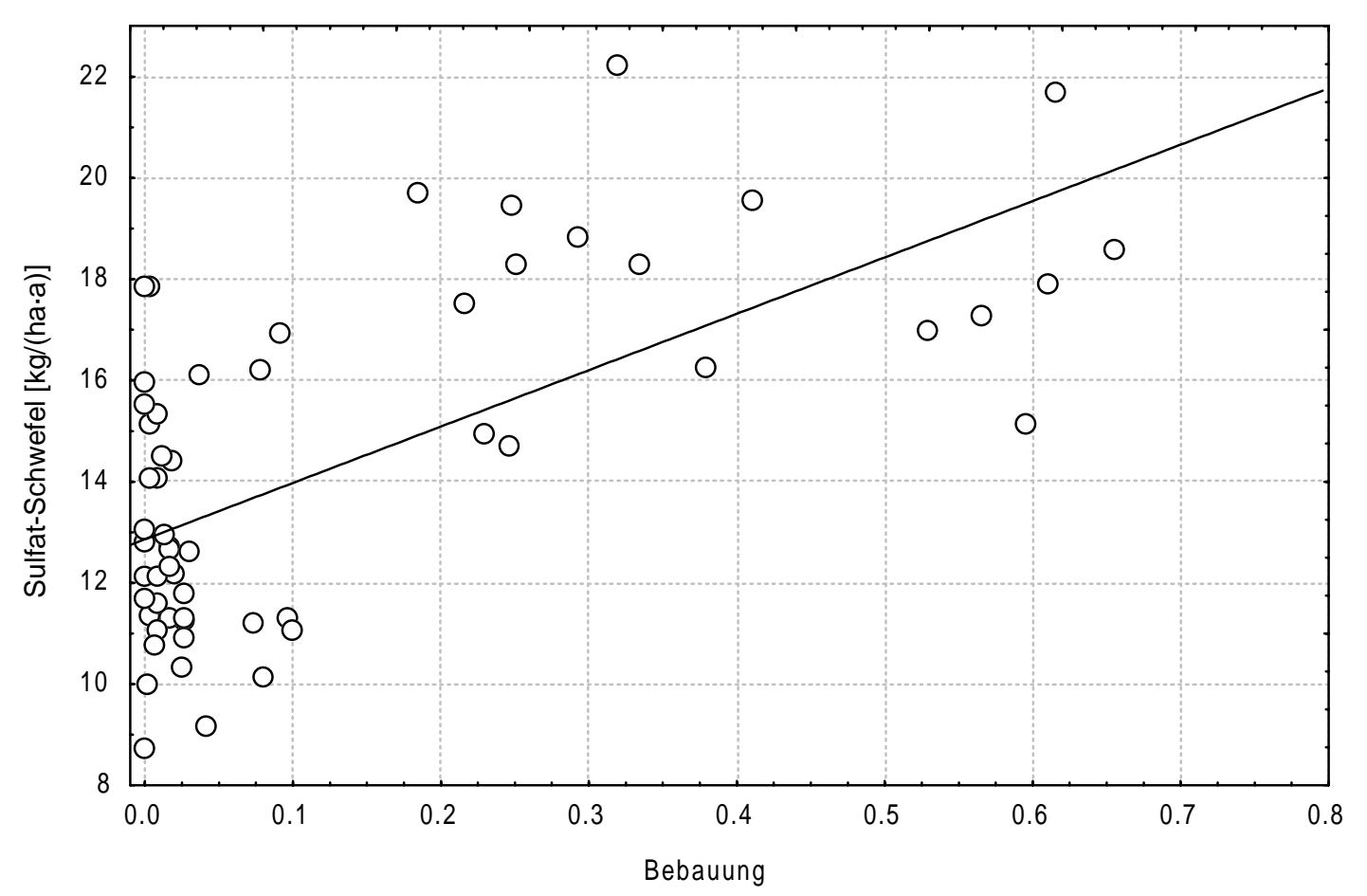

Die beobachtete Abweichung von der angenommenen Linearität wurde nicht als so gravierend angesehen, daß ein nicht-lineares Modell angepaßt wurde. Die Tatsache, daß einige gemessene Einträge um $50 \%$ bis $70 \%$ Bebauung niedriger sind als Meßwerte von Stationen mit $20 \%$ bis $40 \%$ Bebauung, wurde als zufällig angesehen und könnte evtl. auf andere räumliche Variablen zurückgeführt werden.

Die Sulfat-Schwefeleinträge an den Stationen des Freilandkollektivs liegen in einem Bereich von 8,7 bis $22,2 \mathrm{~kg}(\mathrm{ha} \bullet)^{-1}$ (s. Tabelle 34, S. 92). Die theoretisch im GIS erzielbaren Werte - durch Einsetzen der jeweiligen Extreme der räumlichen Variablen im GIS - überschreiten mit einem Maximum von $36,1 \mathrm{~kg}(\mathrm{ha} \bullet)^{-1}$ diesen Bereich deutlich (s. Tabelle 69, S. 176). Die im GIS realisierten Werte des Modells liegen jedoch zwischen 8,2 und $24,1 \mathrm{~kg}(\mathrm{ha} \bullet)^{-1}$. Dies kann als ein durch die Meßdaten abgesicherter Wertebereich angesehen werden.

Die theoretischen und im GIS realisierten Modellwerte für den Protoneneintrag und den pH-Wert sind identisch (s. Tabelle 69). Die Modellwerte für den mittleren pHWert im GIS liegen zwischen 4,2 und 5,4. Der modellierte Protoneneintrag in Niedersachsen wird im GIS mit Werten von 55 bis $589 \mathrm{~g}$ (ha•a) ${ }^{-1}$ umgesetzt. Auch die Meßwerte an den Freilandstationen zeigen einen entsprechenden Wertebereich, weshalb die Modellergebnisse in ihrer Höhe als plausibel angesehen werden. 
Ebenso plausibel erscheinen die Beiträge der räumlichen Variablen $\mathrm{zu}$ den Modellwerten. Vom Westen Niedersachsens bis an seine Ostgrenze ( 340 km) führt so der Regressionskoeffizient für den Rechtswert zu einer Abnahme des $\mathrm{pH}$ Modellwertes um 0,85, der Protoneneintrag wird mit knapp $200 \mathrm{~g}(\mathrm{ha} \bullet)^{-1}$ im Osten höher modelliert als im Westen Niedersachsens. Der Regressionskoeffizient von 0,0004 für die Geländehöhe im Protonenmodell sorgt für eine Beitrag von $0 \mathrm{~g}$ (ha•a) ${ }^{1}$ auf Meereshöhe bis zu $400 \mathrm{~g}\left(\right.$ ha॰a) ${ }^{-1}$ bei einer Geländehöhe von $1000 \mathrm{~m}$ ü. NN. Die Exponiertheit zum Umgebungsminimum (expmin) liefert für den Wert des pHRegressionsmodells einen Beitrag von 0 bis 0,29.

Die Bestimmtheitsmaße für beide Modelle sind höher als das für das AmmoniumStickstoffmodell und zeigen, daß ein großer Teil (über 60\%) der Zielvariablenvarianz durch das Modell erklärt werden kann, der Modellfehler vor allem des Protonenmodells aber ist im Verhältnis zum Mittelwert der Meßergebnisse (C.V.) mit 48,5\% groß (Tabelle 64, S. 137), was die Verwendbarkeit der Regionalisierungsergebnisse als Entscheidungshilfe für umweltrelevante Fragen stark einschränkt.

Zusammenfassend kann festgestellt werden, daß für die Einträge an Sulfat-Schwefel, Ammonium-Stickstoff und Nitrat-Stickstoff plausible Regionalisierungsmodelle hoher Präzision durch eine schrittweise lineare Regression gefunden werden konnten. Der Variationskoeffizient (C.V.) des Chlormodells ist zwar relativ hoch, er liegt aber deutlich unter der Standardabweichung der Meßdaten (vgl. Tabelle 34, S. 92), was die Überlegenheit des Modells gegenüber einer Angabe des Mittelwertes anzeigt. Die Modelle für den $\mathrm{pH}-\mathrm{Wert}$ und mehr noch für den Protoneneintrag der Freilandniederschläge erreichen eine geringere Präzision. Der RMSE des $\mathrm{pH}-$ Wertmodells ist mit 0,2 fast so hoch wie die Standardabweichungen der Meßdaten $(0,3)$, was auch für den RMSE des Protonenmodells beobachtet wird $\left(0,095 \mathrm{~kg} /(\text { ha } \bullet)^{-1}\right.$, Standardabweichung der Meßdaten: $\left.0,122 \mathrm{~kg} /(\text { ha } \bullet)^{-1}\right)$. Dieses Regionalisierungsergebnis ist unbefriedigend.

\subsubsection{Integration der Bestandesdepositionen}

Die Diskussion der Modelle zur Regionalisierung der Anreicherungsfaktoren (Verhältnis Bestandesniederschläge zu Freilanddepositionen) wird recht kurz 
gehalten, da die Frage nach deren Zuverlässigkeit und Repräsentanz für das Zielgebiet wegen des zu geringen Stichprobenumfangs (9 bzw. 10 Stationen) negativ beantwortet werden muß (Kap. 4.2.3). Es bleibt $\mathrm{zu}$ diskutieren, inwiefern die gefundenen Regressionskoeffizienten dem Kriterium der Plausibilität entsprechen und ob die Modelle plausible Wertebereiche liefern.

Alle Modelle verwenden nicht mehr als zwei Regressoren (s. Tabelle 67, S. 139). Schon die Nutzung eines zweiten Regressors ist aber bei nur 9 bzw. 10 Stationen gewagt, denn die Wahrscheinlichkeit von nur zufällig im Datensatz vorhandenen Korrelationen zwischen der Zielvariablen und den Regressoren und damit von Überoder Fehlinterpretationen ist bei einem solch geringen Datenumfang hoch.

Die recht schwachen Bestimmtheitsmaße für die Anreicherungsfaktoren des Protoneneintrags und des $\mathrm{pH}-$ Wertes und der große Modellfehler (RMSE) bei der Regionalisierung des Anreicherungsfaktors für die Protoneneinträge kann zumindest teilweise auf die relativ ungenauen Freilandmodelle zurückgeführt werden, die zusammen mit den mittleren Bestandesniederschlägen zur Herleitung der abhängigen Variablen verwendet wurden (s. Kap. 4.2.4). Auch die Unsicherheiten der Freilandregionalisierung (s. Ende Kap. 6.2.1) gingen so mit in die Bestimmung der abhängigen Variablen eingegangen.

Bei den Modellen für die Anreicherungsfaktoren von Sulfat-Schwefel, NitratStickstoff und Ammonium-Stickstoff sowie für den $\mathrm{pH}-$ Wert sind Dummy-Variablen zur Baumart beteiligt (Kap 3.4.2.1). Fichte koinzidiert mit einer durchschnittlich stärkeren Anreicherung von Nitrat- und Ammonium-Stickstoff und einem durchschnittlich niedrigeren $\mathrm{pH}-$ Wert. Im Fall von Sulfat-Schwefel wurde im Vergleich zu den anderen Baumarten für die Meßstationen unter Buche eine um durchschnittlich $2,5 \mathrm{~kg}(\mathrm{ha} \bullet \mathrm{a})^{-1}$ geringere Anreicherung gegenüber den Freilanddepositionen modelliert.

Die Verwendung von Baumarten oder Baumartengruppen ist bereits aus anderen Regionalisierungen bekannt (s. TOBIAS et al., 1989, LENZ et al., 1996). Auch sie beobachteten für Fichtenbestände allgemeinen höhere Depositionsrisiken, die aber wegen des ordinal ausgerichteten Ansatzes nicht vergleichbar quantifiziert wurden. Auch u. a. von ElLENBERG et al. (1986) wurden für die im Solling gelegene F1Fichtenfläche und die B1-Buchenfläche ähnliche Beobachtungen beschrieben. Dies wird allgemein durch die höhere Filterwirkung - bedingt durch eine größere innere 
Oberfläche und eine ganzjährige Benadelung - des Fichtenbestandes gegenüber dem Buchenbestand begründet und erscheint plausibel.

Außer der Bestockung mit Fichte wird auch die Landnutzungsvariable Acker in den Modellen für den Ammoniumanreicherungsfaktor und den pH-WertAnreicherungsfaktor verwendet. Hier beschreibt Acker die Erhöhung des Ammoniumanreicherungsfaktors für einen Ort mit $100 \%$ landwirtschaftlicher Nutzung um $1,855 \mathrm{~kg}(\mathrm{ha} \bullet \mathrm{a})^{-1}$ (s. Tabelle 67). Der Anreicherungsfaktor für den $\mathrm{pH}-$ Wert wird bei $100 \%$ landwirtschaftlicher Nutzung um 0,244 erhöht. Es wird also in landwirtschaftlich geprägten Gebieten relativ zum Freilandniederschlag mehr Ammonium-Stickstoff in Wäldern deponiert und die Bestandesniederschläge haben einen höheren $\mathrm{pH}-$ Wert als in Gebieten mit geringem Anteil landwirtschaftlich genutzter Fläche. Die Bedeutung landwirtschaftlicher Nutzung, explizit der intensiven Viehhaltung für die Emissionen von Ammoniak - das in wäßriger Lösung basisch zu Ammonium reagiert - ist hinlänglich bekannt (z. B. ISERMANN \& ISERMANN, 1999). Daß durch die basische Reaktion des Ammoniak zu Ammonium eine Pufferwirkung gegenüber stärkeren Säuren und daher ein höhere pH-Wert in den Niederschlagsproben zu finden ist, erklärt den gefundenen Zusammenhang zwischen Acker und pH-Wert plausibel.

Der Abstand zur Küste (Variable der geographischen Lage) ist Teil des Modells für den Anreicherungsfaktor des Chloreintrages. Der negative Regressionskoeffizient beschreibt geringere Anreicherungsfaktoren bei größerem Abstand zur Küste. Eine geringere Anreicherung des Chlors in küstenfernen Gebieten erscheint angesichts der Abhängigkeit der trockenen Deposition von der Spurenstoffkonzentration (s. ( 23 ) in Kap. 4.2.1) plausibel, da man von geringeren Konzentrationen des meeresbürtigen Chlors in küstenfernen Gebieten ausgehen kann.

Der durchschnittliche Jahresniederschlag und die Luv (höhenabhängige Variablen) waren an den Modellen zur Anreicherung von Sulfat-Schwefel, Chlor, Protonen bzw. Nitrat-Stickstoff beteiligt (Tabelle 67, S. 139). Die Luvlage eines Standortes führt nach ToBiAs et al. (1989) und WIEDEY (1987) (in ToBIAS et al. 1989) zur Erhöhung von Einträgen gegenüber Leelagen. Dieser Effekt konnte für den Anreicherungsfaktor des Eintrags an Nitrat-Stickstoff beobachtet und quantifiziert werden. Für alle anderen Stoffe wurde dieser Effekt nicht gefunden. Wegen des 
geringen Datenumfangs kann seine Wirksamkeit jedoch nicht ausgeschlossen werden kann.

Für den Jahresniederschlag wurden in den Modellen für die Anreicherungsfaktoren von Sulfat-Schwefel, Chlor, Protonen und den $\mathrm{pH}-$ Wert positive Regressionskoeffizienten gefunden. Erhöhte Auswaschung der genannten Stoffe aus der Atmosphäre in Gebieten hoher Niederschläge erscheint plausibel. Da die Variable Jahresniederschlag maßgeblich von der Geländehöhe geprägt ist, können hinter diesem Zusammenhang zusätzlich auch andere Effekte vermutet werden. So werden erhöhte Nebelhäufigkeit oder Inversionslagen $300 \mathrm{~m}$ bis $500 \mathrm{~m}$ über Talgrund (erhöhte Spurenstoffkonzentrationen) von TOBIAS et al. (1989) als Gründe für erhöhte Depositionen genannt, die im Zusammenhang mit der Geländehöhe stehen. Entsprechende Einflüsse und Zusammenhänge können aber bei der bestehenden Datenlage nicht überprüft werden.

Ein weiterer Punkt, der neben der Plausibilität der Regressionskoeffizienten diskutiert werden soll, ist die Plausibilität der Modellwerte. In Tabelle 70 sind hierzu die theoretisch möglichen und die im GIS realisierten Minima und Maxima der Modellwerte aufgeführt. Zusätzlich wurden in dieser Tabelle auch die von TOBIAS et al. (1989) erwähnten Minima und Maxima von Anreicherungsfaktoren verschiedener Quellen aufgenommen. Als weiterer Maßstab wurden für alle verfügbaren jährlichen Depositionen der Waldmeßstationen in Niedersachsen Anreicherungsfaktoren berechnet und deren Extremwerte in die Tabelle integriert.

Tabelle 70: Theoretisch mögliche und im GIS realisierte Minima und Maxima der Modelle für die Bestandesanreicherungsfaktoren; zusätzlich die von TOBIAS et al. (1989) erwähnten und die für alle Waldmeßwerte berechneten Minima und Maxima der Anreicherungsfaktoren

\begin{tabular}{l|rr|rr|rr|rr}
\hline & \multicolumn{2}{|c|}{ theoretisch möglich } & \multicolumn{2}{c|}{ im GIS realisiert } & \multicolumn{2}{|c|}{ ToBIAS et al. (1989) } & \multicolumn{2}{c}{ Meßwerte in Nds. } \\
& Min. & Max. & Min. & Max. & Min. & Max. & Min. & Max. \\
\hline Sulfat-S & $\mathbf{- 1 , 4}$ & 9,3 & $\mathbf{- 1 , 1}$ & 9,3 & 1,5 & 6 & 1,2 & 10,7 \\
Nitrat-N & 0,8 & 11,5 & 0,8 & 10,6 & 1,7 & 2,8 & 0,5 & 9,5 \\
Ammonium-N & 1,3 & 5,1 & 1,3 & 5,1 & 1,0 & 3,1 & 0,4 & 5,6 \\
Chlor & $\mathbf{- 1 5 , 3}$ & 35,6 & $\mathbf{- 1 2 , 4}$ & 28,7 & & & 1,0 & 13,2 \\
$\mathbf{H}^{+}$ & $\mathbf{- 0 , 9}$ & 8,4 & $\mathbf{- 0 , 9}$ & 8,4 & 1,0 & 4,0 & & \\
\hline $\mathbf{p H}$ & 0,8 & 1,1 & 0,8 & 1,1 & & & & \\
\hline
\end{tabular}

Die für Sulfat-Schwefel, Chlor und den Protoneneintrag ermittelten Modelle führen theoretisch und durch die Realisierung im GIS zu negativen Werten! Das ist per se völlig unplausibel und nicht annehmbar. Negative Werte beschreiben einen Austrag 
des Zielelementes mit dem Bestandesniederschlag, was schlichtweg unmöglich und daher unplausibel ist.

Sehr hoch erscheint der Maximalwert für den Chloranreicherungsfaktor von 35,6 (theoretisch erreichbar) bzw. 28,7 (im GIS ermittelt). Die Meßwerte erreichten allerdings immerhin auch ein Maximum von 13,2, immerhin fast halb so hoch wie das Maximum der im GIS realisierten Modellwerte. Die Wertebereiche der Modelle für die Anreicherungsfaktoren von Nitrat- und Ammonium-Stickstoff befinden sich zumindest im Rahmen der für die niedersächsischen Waldmeßstationen berechneten Anreicherungsfaktoren. Die von TOBIAS et al. (1989) erwähnten Anreicherungsfaktoren bewegen sich allerdings in einem engeren Wertebereich, sie erreichen vor allem kleinere Maxima.

Die Ergebnisse der Regionalisierung der Anreicherungsfaktoren von Freilanddepositionen $\mathrm{zu}$ Bestandesniederschlägen müssen größtenteils als unplausibel eingestuft werden. Obwohl die Regressionskoeffizienten plausible Vorzeichen und Werte haben, zeigt die Umsetzung der Regressionsmodelle im GIS, daß teilweise unplausible Werte geschätzt werden. Dies muß zuallererst auf die geringe Anzahl von Meßstationen mit einer kompletten Meßreihe im untersuchten Zeitraum zurückgeführt werden. Die Tatsache, daß auch die Fehler der Freilandmodelle, die Berechnungsgrundlage der hier verwendeten Zielvariablen sind, mit in die Anreicherungsmodelle eingehen, wird demgegenüber als gering eingeschätzt.

Nur für einen Zeitraum von 3 Jahren oder weniger wäre für das Untersuchungsgebiet die Integration von Daten weiterer Meßstationen in die Analyse möglich gewesen. Je kürzer aber der zugrundeliegende Zeitraum ist, desto größer ist der Einfluß einzelner Monats- bzw. Jahresmeßwerte im Rahmen der Mittelwertbildung. Je kürzer der Beobachtungsraum, desto häufiger gehen Stationswerte in die Untersuchungen ein, die nicht als typisch für den Meßort angesehen werden können.

$\mathrm{Zu}$ den bisher genannten Problemen bei der GIS-technischen Umsetzung der Modelle kommt das Fehlen einer flächendeckenden digitalen Informationslage über die stockenden Baumarten. Die Dummy-Variablen Fichte und Buche können momentan nur als Nadel- bzw. Laubbaumbestand durch das Landnutzungsraster umgesetzt werden. Da die Baumart aber das wohl größte Erklärungspotential für 
Anreicherungsfaktoren hat (s.a. TOBIAS et al., 1989), ist eine baldige Digitalisierung von Informationen der Forsteinrichtung erstrebenswert. 


\section{Zusammenfassung}

Die Regionalisierung von Klimaelementen und atmogenen Depositionen durch Beschreibung der Meßsituation und der räumlichen Autokorrelation bei Beachtung der drei Kriterien der Einfachheit, Präzision und Plausibilität war Ziel der vorliegenden Arbeit. Insbesondere die Möglichkeiten, die sich durch die Verwendung eines Geographischen Informationssystems (GIS) für die Unterstützung des Regionalisierungsprozesses ergeben, sollten aufgezeigt werden.

Zur Beschreibung der Meßsituation an den jeweiligen Meßstationen wurden räumliche Variablen verwendet (s. Kap. 3). In Form von Rastern lagen diese räumlichen Informationen in einem GIS vor und konnten bei bekannter geographischer Lage für die Meßstationen ermittelt bzw. aus den Rasterwerten interpoliert werden. Gegenüber einer möglicherweise genaueren Messung an den Meßstellen hat diese Vorgehensweise den Vorteil, daß die zur Beschreibung der Meßsituation und zur Modellbildung verwendeten räumlichen Variablen für die spätere Umsetzung der Modelle im GIS für das gesamte Zielgebiet zur Verfügung stehen.

Die zur Beschreibung der Meßsituation verwendeten räumlichen Variablen lassen sich in drei Kategorien einteilen: Variablen der geographischen Lage (Rechtswert, Hochwert, Abstand zur Küste), höhenabhängige Variablen (Geländehöhe, Hangneigung, Hangrichtung, Exponiertheit zum Umgebungsminimum, Luv, Leewirbel, Lee-Effekt 50, etc.) und Variablen aus Kartierungen bzw. Klassifizierungen der Fernerkundung (z. B. Anteil von Bebauung, Wald, landwirtschaftlich genutzten Flächen bzw. Acker oder Gewässern an der Landnutzung). Die zu den Variablen aus Kartierungen zu zählenden Variablen der Forsteinrichtung konnten im Rahmen dieser Arbeit nicht genutzt werden. Sie sind nicht flächendeckend für alle Waldbestände verfügbar, eine Recherche für die einzelnen Meßpunkte ist mit Unzulänglichkeiten behaftet und auch das Problem der unterschiedlichen Forsteinrichtungszeitpunkte kann im Rahmen der Regionalisierung noch keine Berücksichtigung finden. Die Fortschreibung von Forsteinrichtungsdaten durch entsprechende Wuchsmodelle und die zunehmende Digitalisierung von 
Forsteinrichtungsdaten könnten jedoch zu neuen Nutzungsmöglichkeiten im Rahmen der Regionalisierung führen.

Die räumlichen Variablen wurden als Regressoren in schrittweisen linearen Regressionen verwendet (s. Kap. 2.5). Der systematische Teil der räumlichen Streuung der Zielvariablen (Niederschlagssumme, Lufttemperatur, relative Luftfeuchte und Depositionen) sollte durch die Streuung der räumlichen Variablen erklärt werden. Um dabei eine Fehl- oder Überinterpretation der vorliegenden Datenkollektive $\mathrm{zu}$ vermeiden und damit die Gültigkeit der modellierten Beziehungen für das Zielgebiet sicherzustellen, wurde den Kriterien Einfachheit und Plausibilität in der Modellbildung große Beachtung geschenkt. Zunächst geschah dies durch die Integration ausschließlich solcher Regressoren, die das Bestimmtheitsmaß des Regressionsmodells um mindestens $5 \%$ verbessern konnten. Dadurch kann nur eine kleine Zahl von Regressoren in die Modelle aufgenommen werden. Die gefundenen Zusammenhänge wurden zudem vor dem bekannten Wissen auf Plausibilität untersucht. Unplausible Vorzeichen der Regressionskoeffizienten führten trotz evtl. gegebener Präzision bei der Beschreibung der Daten zur Entnahme des entsprechenden Regressors aus dem Modell.

Zielvariablen waren allgemein langjährige Mittelwerte von Klimaelementen und Depositionen. Daten des Deutschen Wetterdienst (DWD; Niederschlagssummen, Lufttemperatur, relative Luftfeuchte) wurden dabei durch Daten der Harzwasserwerke (HWW; Niederschlagsdaten) und des Institutes für Forstliche Bioklimatologie aus dem Harz ergänzt (TRANSEKT-Datenkollektiv zur Lufttemperatur). Die Depositionsdaten wurden vom Niedersächsischen Landesamt für Ökologie (NLÖ) und vom Forschungszentrum Waldökosysteme der GeorgAugust-Universität Göttingen zur Verfügung gestellt.

Je länger der Zeitraum ist, welcher der Mittelwertbildung zugrunde liegt, desto geringer ist der Einfluß einzelner Wetter- bzw. Depositionsereignisse auf den Mittelwert. Damit steigt der Einfluß der Meßsituation auf die Ausprägung des Mittelwertes, was bei der gewählten Vorgehensweise wünschenswert ist.

Im Falle der Regionalisierung dreißigjähriger mittlerer Niederschlagssummen konnte wegen einer ausreichend großen Anzahl und einer geeigneten räumlichen Verteilung von Meßstationen im Anschluß an die schrittweise lineare Regression eine 
Semivariogrammanalyse und das geostatistische Kriging der Regressionsresiduen durchgeführt werden (s. Kap. 5.1.2).

Die Tatsache, daß nur die Daten für die langjährigen durchschnittlichen Niederschlagssummen für geostatistische Untersuchungen geeignet waren, verdeutlicht die heterogene Datensitutation bezüglich der untersuchten Zielvariablen. Die Eignung der Datenkollektive zur Regionalisierung mußte daher individuell überprüft werden. Über die Betrachtung des Datenumfangs und der räumlichen Verteilung der Meßstationen hinaus konnte eine GIS-gestützte quantitative Untersuchung der Meßnetzrepräsentanz unter Verwendung der räumlichen Variablen zur Beschreibung der Meßsituation durchgeführt werden. Diese ergab für einige Datenkollektive z.T. beachtenswerte Repräsentanzmängel für das Zielgebiet. So wurde beispielsweise für die Datenkollektive des Deutschen Wetterdienstes (DWD) zur durchschnittlichen Lufttemperatur und zur durchschnittlichen relativen Luftfeuchte eine Unterrepräsentanz für Meßsituationen an Orten mit starker Hangneigung festgestellt. Auch die Tatsache, daß fast alle DWD-Stationen ortsnah bzw. in Ortschaften gelegen sind, ist hier als ein Mangel an Repräsentanz zu nennen, der zumindest teilweise durch die Integration des zweiten Datenkollektivs zur Lufttemperatur (TRANSEKT) ausgeglichen werden konnte. Die meisten räumlichen Variablen betreffend konnte Repräsentanz festgestellt werden, der Anteil nicht repräsentierter Flächen an der Gesamtfläche des Zielgebiets konnte mit Hilfe des GIS geschätzt werden. In den meisten Fällen werden nur sehr kleine Teile des Zielgebietes nicht durch die verwendeten Datenkollektive repräsentiert.

Das Zielgebiet Niedersachsen wurde grundsätzlich in Berg- und Tiefland unterteilt. Für eine solche Unterteilung spricht zunächst das Kriterium der Plausibilität. Die Verschiedenheit der orographischen Verhältnisse in den Untergebieten läßt sich durch die räumlichen Variablen beschreiben und Auswirkungen auf eine regressionsgestützte Regionalisierung erwarten. Die Modelle der Untergebiete zeichneten sich zudem vielfach durch eine präzisere Beschreibung der Daten, vor allem aber auch durch eine konsistentere und einfachere Modellbildung aus.

Die GIS-gestützte Umsetzung der Regionalisierungsmodelle in Form von Rastern konnte sowohl zur Darstellung der Ergebnisse als auch zur Überprüfung der Plausibilität verwendet werden. Die Darstellung der Ergebnisse ist nicht immer dazu geeignet, das Vertrauen in die Regionalisierungsmodelle zu stärken. Durch die 
vereinfachende Wirkung der Regressionsmodelle erscheinen die graphischen Darstellungen oftmals zu einfach und schematisch. Die räumliche Verteilung einer jeden Zielvariablen, die eine Realisierung z.T. sehr komplexer Prozesse darstellt, wird schließlich auf die räumliche Verteilung weniger Variablen zurückgeführt. Bei näherer Betrachtung und Kenntnis der Methodik und Datenlage, die zu diesen Regionalisierungsmodellen führen, können diese Darstellungen jedoch richtig interpretiert werden und als wichtiges Hilfsmittel zur Entscheidungsfindung dienen.

\subsection{Klimaelemente}

Durch die schrittweise lineare Regression der Zielvariablenvarianzen auf die Streuungen von räumlichen Variablen konnten einfache, plausible Regressionsmodelle hoher Präzision für die Regionalisierung der untersuchten Klimaelemente gefunden werden. Es wurden sowohl für die einzelnen Zielvariablen Lufttemperatur, Niederschlagssumme und relative Luftfeuchte (jeweils durchschnittliche Monatsund Jahreswerte für den Beobachtungszeitraum 1961 bis 1990) als auch für die Untergebiete Bergland und Tiefland spezifische und typische Beziehungen zu den räumlichen Variablen gefunden und durch die Regressionsmodelle beschrieben. Diese Zusammenhänge gelten nur für das untersuchte Gebiet und ausschließlich für den durch die Daten repräsentierten Zeitraum. Extrapolationen können bestenfalls den Charakter von Szenarien haben. Prognosen im Sinne des klimatischen Wandels (,climate change“) sollten über prozessanalytische Modelle getroffen werden. Empirisch-statistische Untersuchungen wie die vorliegende können beispielsweise zur Beschreibung bestehender Wuchsbedingungen Verwendung finden.

Die Unterschiedlichkeit der Untergebiete ließ sich über die Rasterwerte der räumlichen Variablen beschreiben und wurde von ScHULZ (in Vorb.) zur clusteranalytischen Definition einer objektiven Grenze zwischen Berg- und Tiefland genutzt. Diese Grenze wurde zur graphischen Darstellung der gefundenen Modelle verwendet. Eine $10 \mathrm{~km}$ lange Übergangszone von der Grenze ins (größere) Tiefland hinein wurde dabei zur Vermeidung möglicher abrupter Sprünge von den Modellwerten im Bergland $\mathrm{zu}$ denen im Tiefland definiert. Die graphischen 
Darstellungen der Modellergebnisse zeigen für viele Modelle ohnehin keine großen Sprünge zwischen den Modellwerten.

Die Regressionsmodelle zur Regionalisierung der Lufttemperatur zeigen bei hoher Präzision bezüglich ihrer Zusammensetzung deutliche Unterschiede für Berg- und Tiefland. Die räumliche Verteilung der Lufttemperatur im niedersächsischen Tiefland kann zu einem großen Teil auf die Streuung von lageabhängigen Variablen (Rechtswert, Hochwert und Abstand zur Küste), von Landnutzungsvariablen (Bebauung und Gewässer) und von höhenabhängigen Variablen (Lee-Effekt 25 und Exponiertheit zum Umgebungsminimum) zurückgeführt werden (s. Tab. Tabelle 46, S. 112). Die Variablen der erstgenannten Variablengruppen haben dabei einen größeren Einfluß auf die Ausprägung der Zielvariablen als die beiden höhenabhängigen Variablen. In den Berglandmodellen hingegen kommt der dominierende Einfluß der Geländehöhe auf die Lufttemperatur zum Ausdruck. Nur durch die integrierte Analyse eines Datenkollektivs des Institutes für Bioklimatologie konnte zusätzlich der Einfluß von Kaltluftabfluß auf die durchschnittliche Lufttemperatur gefunden und beschrieben werden.

Auch die Berglandmodelle für die räumliche Verteilung der durchschnittlichen monatlichen und jährlichen Niederschlagssummen werden durch die Geländehöhe geprägt. Das hohe Erklärungspotential dieser Variable führt dazu, daß außer ihr nur die höhenabhängigen Variablen Leewirbel und Luv sowie im Monat August die lageabhängige Variable Hochwert in die Regressionsmodelle Eingang fanden. Die Regressionsmodelle für die Niederschlagsverteilung im niedersächsischen Tiefland sind aus den Variablen Geländehöhe, Rechtswert und Abstand zur Küste zusammengesetzt, wobei die Geländehöhe zwar an jedem monatlichen Modell beteiligt ist, stets aber einen geringeren Anteil am Bestimmtheitsmaß liefert als die beteiligten lageabhängigen Variablen. Sowohl die Modelle für das Bergland als auch diejenigen für das Tiefland zeigen Fehler (rooted mean squared error, RMSE), die im Verhältnis zu den möglichen Meßfehlern bei Niederschlagsmessungen sehr gering sind (s. S. 74).

Die Regressionsresiduen waren im Fall der Niederschlagssummen Gegenstand einer geostatistischen Untersuchung. Durch Kreuzvalidierung konnte gezeigt werden, daß das geostatistische Kriging der Regressionsresiduen die Schätzungen der Zielvariablen gegenüber reiner Regression vor allem im Tiefland bedeutend 
verbessert. Eine lokale Meßnetzverdichtung im Harz durch die Integration von Daten der Harzwasserwerke führt zu Schwierigkeiten bei der Semivariogrammanalyse für das Bergland. Die Semivariogramme des Tieflandkollektivs zeigen hingegen eine typische sphärische Form und lassen keine Abweichungen von der vorausgesetzten Stationarität erwarten.

Auch zur Regionalisierung der relativen Luftfeuchte wurden schrittweise lineare Regressionsmodelle angepaßt. Sie zeigen deutliche Unterschiede zwischen Berg- und Tiefland. In der Zusammensetzung zeigen sie große Ähnlichkeit mit den Modellen für die Regionalisierung der Lufttemperatur, was auf die enge Beziehung zwischen diesen beiden Klimaelementen hinweist. Während in den Berglandmodellen entweder die höhenabhängige Variable Lee-Effekt 50 oder die Geländehöhe selbst den größten Anteil an der Aufklärung der räumlichen Streuung der relativen Luftfeuchte haben, werden die Modelle im Tiefland durch die Landnutzungsvariable Bebauung und durch die lageabhängigen Variablen Hochwert bzw. Abstand zur Küste geprägt.

Die geringe Aufklärung der Zielvariablenvarianz durch einige Monatsmodelle zeigt sich in geringen Werten für das Bestimmtheitsmaß und höheren RMSE-Werten, die bei $45 \%$ bis $90 \%$ der Standardabweichungen der Meßwerte liegen. Eine Regionalisierung läßt bei derart hohen Werten nur eine geringfügige Verbesserung der Schätzgenauigkeit gegenüber einer Beschreibung der räumlichen Verteilung durch den Mittelwert der Meßdaten erwarten. Sie sollte dennoch auch bei geringer Verbesserung der Präzision wegen des erzielten Erkenntnisgewinns bezüglich der modellierten Zusammenhänge bevorzugt werden.

Für die Berglandmodelle liegt das Verhältnis zwischen RMSE-Werten und Standardabweichungen der Meßwerte zwischen $30 \%$ und $67 \%$. Eine deutlich genauere Regionalisierung als durch Mittelwertbildung ist somit zu erwarten.

Für die drei hier regionalisierten Klimaelemente konnten auf einheitlichem Wege einfache und plausible Regionalisierungsmodelle gefunden werden, die in den meisten Fällen Schätzungen mit erheblich höherer Genauigkeit im Vergleich zur Mittelwertbildung erwarten lassen. Eine höhere Präzision der Modelle könnte eventuell über die detailliertere Beschreibung von Naturphänomenen durch alternative räumliche Variablen erreicht werden. Ohne Datenkollektive, die zur Validierung der Modelle geeignet sind, ist die Überinterpretation, die zu starke 
Anpassung an die vorliegenden Daten, bei einer solchen Vorgehensweise jedoch eine große Gefahr für die Gültigkeit der Modelle. Vielversprechender erscheint die Berücksichtigung von extremen Meßstandorten bei der zukünftigen Planung und Unterhaltung von Meßnetzen, um die Variabilität der Meßsituationen weiter an die des gesamten Zielgebietes angleichen zu können.

Ausgehend von den hier bereits regionalisierten langjährigen Mittelwerten von Niederschlagssummen, Lufttemperatur und relativer Luftfeuchte können durch die Verwendung des GIS auf einfachem Wege weitere Informationslagen abgeleitet werden. Die wohl grundlegendste Ableitung für (forst-)ökologische Zwecke ist die Länge der Vegetationsperiode. Hierbei kann für jeden Rasterpunkt eines Ergebnisrasters durch eine lineare Interpolation zwischen den regionalisierten Monatsmittelwerten der Lufttemperatur, deren Gültigkeit am 15. eines jeden Monats vorausgesetzt wird, ein Temperaturverlauf für das gesamte Jahr simuliert werden. Für jeden Schwellenwert wie etwa $8^{\circ} \mathrm{C}$ oder $10{ }^{\circ} \mathrm{C}$ kann dadurch die Zahl der Tage ermittelt werden, deren Mitteltemperatur über dem Schwellenwert liegt. Vergleiche mit dem Verlauf der gemessenen Tagesmitteltemperaturen des TRANSEKTDatenkollektivs bestätigten die Eignung dieses linearen Interpolationsansatzes (SABOROwSKI, mdl. Mitteilung 1998). Ausgehend von der Länge der Vegetationsperiode können weitere Größen abgeleitet werden wie z. B. die Niederschlagssumme in der Vegetationsperiode oder die Temperatursumme in der Vegetationsperiode. Ein Ansatz zur GIS-gestützten Wuchsraumgliederung auf Basis von regionalisierten Klimaparametern wird von JANSEN et al. (in Vorb.) vorgestellt.

\subsection{Depositionen}

Die Regionalisierung durchschnittlicher jährlicher Bulk-Depositionen von SulfatSchwefel, Nitrat-Stickstoff, Ammonium-Stickstoff, Chlor, Protonen und des pHWertes wurde geprägt durch die geringe Anzahl von Messungen - insbesondere in Waldbeständen - im Untersuchungsgebiet. Als Konsequenz aus dieser Tatsache ist die Beschränkung der Analyse auf Mittelwerte für den (kurzen) Zeitraum von 1986 bis 1990 zu verstehen. Dieser Zeitraum von sechs Jahren stellt einen Kompromiß dar 
zwischen der gewünschten Reduzierung des Einflusses einzelner Wetter- und Depositionsereignisse durch eine möglichst langfristige Betrachtungsweise und der geringen Anzahl an Meßstationen.

Für die modellierten Freilanddepositionen standen zur Regionalisierung Daten von ausreichend vielen Stationen zur Verfügung. Die Freilanddatenkollektive können weitgehend als repräsentativ für das Zielgebiet angesehen werden. Dies gilt auch nach der Trennung in Berg- und Tieflandkollektiv, wenn auch in etwas eingeschränktem Maße.

Die Verteilungen der untersuchten Stoffe konnten zumindest teilweise durch diejenigen der räumlichen Variablen erklärt werden. Die Bestimmtheitsmaße und Werte des RMSE zeigen, daß die Verteilungen der Depositionsdaten unterschiedlich gut durch die Modelle abgebildet werden. Bestimmtheitsmaße von 12,2\% (NitratStickstoff Tiefland) bis 92,8\% (Chlor Tiefland) werden erreicht. Die trotz hoher Bestimmtheitsmaße erstaunlich hohen RMSE-Werte und Variationskoeffizienten (C.V.) der Chlormodelle für das Tiefland und für ganz Niedersachsen können durch die extrem hohen Chloreinträge an drei Stationen in Nähe der Nordsee erklärt werden. Diese Struktur der Daten kann zu einem großen Teil auf den Regressor Gewässer zurückgeführt werden. Der Fehler des Modells (RMSE) ist jedoch vor allem in Relation zum Mittelwert der Meßdaten, der hauptsächlich durch die vielen niedrigen Meßwerte geprägt ist, relativ groß.

Außer den hohen Bestimmtheitsmaßen der Chlormodelle und dem besonders niedrigen des Tieflandmodells für den Nitrat-Stickstoff erreichen die anderen Modelle Bestimmtheitsmaße zwischen 47,7\% und 66,4\%, was als zufriedenstellend angesehen werden kann. Der Variationskoeffizient zeigt - außer für die Protonenmodelle und den Chloreintrag im Tiefland - Werte von 11,9\% bis 15,0\%. Vergleicht man diese Werte mit den möglichen Meßfehlern, die noch erheblich über denen der Niederschlagsmessungen liegen müssen (s. Tabelle 21, S. 74), so kann dies als zufriedenstellend angesehen werden.

Die Regionalisierung von Anreicherungsfaktoren für die Waldmeßdaten konnte wegen des zu geringen Datenumfangs nicht zu sicheren Ergebnissen führen. Es entstanden zwar zumindest teilweise plausible und auch hoch bestimmte Modelle, eine Gültigkeit der Regressionsmodelle zur Regionalisierung für die Fläche ganz Niedersachsens kann aber wegen des zu geringen Datenumfangs nicht angenommen 
werden. Eine sinnvolle Regionalisierung von Bestandesniederschlägen, Anreicherungsfaktoren oder Gesamtdepositionen in Waldbeständen ist nur bei größerem Datenumfang möglich. Dieser kann derzeit nur durch die Aggregation mehrerer Datenkollektive für orographisch zu definierende Großräume (Tiefland, Mittelgebirge, Alpenvorland, etc.) über Ländergrenzen hinweg erreicht werden. Die Recherche der Meßsituationen dürfte dabei, wie von TOBIAS et al. (1989) beschrieben, ein großes Problem darstellen. 


\section{Literaturliste}

AG-Deposition der Ökosystemforschungszentren (1994): Gemeinsames Konzept der langfristigen Erfassung von Stofftransporten zwischen terrestrischen Ökosystemen und der Atmosphäre : Workshop der AG Deposition der Ökosystemforschungszentren im Schloß Nienover, Solling, 24.2.-27.2.1994 Protokoll / Hrsg.: AG-Deposition der Ökosystemforschungszentren. Berichte d. Forschungszentrums Waldökosysteme d. Univ. Göttingen, Reihe B 41

Arbeitskreis Standortkartierung (1985): Forstliche Wuchsgebiete und Wuchsbezirke in der Bundesrepublik Deutschland; Arbeitskreis Standortkartierung in der Arbeitsgemeinschaft Forsteinrichtung; Landwirtschaftsverlag MünsterHiltrup, Münster

Ashraf, M., J.C. Loftis und K.G. Hubbard (1997): Application of Geostatistics to evaluate Partial Weather Station Networks. Agricultural and Forest Meteorology 84: 255-271

Banzhaf, E. (1994): Die Regionalisierung der Wärmebelastung in Mainfranken anhand von Geofaktoren; Freiburger Geographische Hefte, Herausgegeben von Hermann Goßmann, Heft 43

Becker, A. (1992): Methodische Aspekte der Regionalisierung; in: Kleeberg, H.B. [Hrsg.], Regionalisierung in der Hydrologie, Ergebnisse von Rundgesprächen der DFG, VCH Weinheim

Berke, O. und U. Busch (1994): Statistische Analyse von räumlichen und zeitlich verteilten Daten. Aus: Tagungsberichte Arbeitsgruppe Ökologie Deutsche Region der internat. Biometrischen Gesellschaft, AG Tagung beim 40. Biometrischen Kolloquium in Münster.

Berke, O. (1998): Über die statistische on-line Prädiktion von Umweltmonitoringdaten im Rahmen von dynamischen linearen Raum-Zeit Modellen. 
Diss. Zur Erlangung des Grades eines Doktors der Naturwissenschaften; Fachbereich Statistik der Unv. Dortmund

Bill, R. und D. Fritsch (1996): Grundlagen der Geo-Informationssysteme; Herbert Wichmann Verlag, Hüthig GmbH, Heidelberg

Blennow, K. und P. Persson (1998): Modelling local-scale frost variations using mobile temperature measurements with a GIS; Agricultural and Forest Meteorology 89: 59-71

Böhner, J. (1996): Säkulare Klimaschwankungen und rezente Klimatrends Zentralund Hochasiens; Göttinger Geographische Abhandlungen; Heft 101; Verlag Erich Goltze GmbH \& Co. KG, Göttingen

Bredemeier, M. (1987): Stoffbilanzen, interne Protonenproduktion und Gesamtsäurebelastung des Bodens in verschiedenen Waldökosystemen Norddeutschlands; Berichte d. Forschungszentrums Waldökosysteme/Waldsterben d. Univ. Göttingen, Reihe A 33

Burga, C.A. und R. Perret (1998): Vegetation und Klima der Schweiz seit dem jüngeren Eiszeitalter; $\mathrm{OH}$ Verlag, Thun

Burkhard, J. (1994): Dünne Wasserfilme auf Fichtennadeln und ihr Einfluß auf den Stoffaustausch zwischen Atmosphäre und Pflanze. In: AG-Deposition der Ökosystemforschungszentren

Busch, U. (1994): Modellierung der Nassen Deposition in Abhängigkeit von der Witterung. Aus: Tagungsberichte Arbeitsgruppe Ökologie Deutsche Region der internat. Biometrischen Gesellschaft, AG Tagung beim 40. Biometrischen Kolloquium in Münster.

Büttner, G., N. Lamersdorf, R. Schultz und B. Ulrich (1986): Deposition und Verteilung chemischer Elemente in küstennahen Waldstandorten. Berichte d. Forschungszentrums Waldökosysteme/Waldsterben d. Univ. Göttingen, Reihe B 1

Chamberlain, A. (1975): Pollution in Plant Canopies. Aus: SHRINER, D.S. et al. (Eds.) 1980: Atmospheric Sulfur Deposition: Environmental Impact and 
Health Conseqents of Atmospheric Sulfur Deposition. Ann Arbor Science Publishers Inc., Gathlinburg, Tenn.

Cressie, N. (1991): Statistiscs for Spatial Data; Wiley, New York

Dozier, J. und J. Frew (1990): Rapid calculation of terrain parameters for radiation modeling from digital elevation data, IEEE Trans. Geosci. Remote Sensing 28 (5): $963-969$

DWD (1980): Anleitung für die Beobachter der Klimahauptstationen des Deutschen Wetterdienstes; Deutscher Wetterdienst Offenbach A.M. - 8., neubearbeitete Auflage.

Ellenberg, H., R. Mayer und J. Schauermann (Hrsg) (1986): Ökosystemforschung Ergebnisse des Solling-Projekts; 1966-1986 / Heinz Ellenberg; Robert Mayer; Juergen Schauermann [Hrsg.]. Stuttgart : Ulmer

ESRI (1990): Understanding GIS, the ARC/Info Method. Environmental Systems Research Institute, Inc., Redlands,CA. USA

ESRI (1995): Arc/Info Version 7-Online-User's Guide. Environmental Systems Research Institute, Inc., Redlands,CA. USA

Felbermeier, B. (1994): Die klimatische Belastbarkeit der Buche; Forstw. Cbl. 113: $152-174$

Fränzle, O., W. Schröder, E. Wildförster, C.-D. Garbe und L. Vetter (1989): Synoptische Darstellung möglicher Ursachen der Waldschäden: Untersuchungen zu Langzeitwirkungen von Kompensationskalkungen auf Buchen-, Fichten- und Kiefernforstökosysteme. Geographisches Institut, Universität Kiel; Umweltbundesamt, Berlin; FE-Vorhaben Nr. : 10803 $046 / 13$

Friedrich (1991): Beiträge zur Methodik der Waldökosystemforschung; J. Friedrich; G. Völker [Hrsg.]. Berichte d. Forschungszentrums Waldökosysteme d. Univ. Göttingen, Reihe B 24 
Führer, H.-W., H.-M. Brechtel, H. Ernstberger, und Ch. Erpenbeck (1988): Ergebnisse von neuen Depositionsmessungen in der Bundesrepubilk Deutschland und im benachbarten Ausland; Mitteilungen des Deutschen Verbandes für Wasserwirtschaft und Kulturbau, Heft 14

Geiger, R. (1961): Das Klima der bodennahen Luftschicht; Vieweg, Braunschweig

Goßmann, H., E. Banzhaf, und G. Klein (1993): Regionalisierung ökologischer Daten - alte Aufgaben, neue Lösungswege; Das Freiburger Regionalisierungsmodell FREIM; Würzburger Geographische Arbeiten 87: 391-417, Würzburg 1993

Graf, J. und S. Schmid (1989): Modellmäßige Erfassung des Transports von Luftverunreinigungen in Regionen mit orographischer Gliederung am Beispiel Nordost-Bayern; Arbeitsgruppe Theoretische Meteorologie, Meteorologisches Institut, Universität München; Umweltbundesamt; Berlin 1989; UBA FB 89098

Gravenhorst, mdl. Mitteilung (1999): Diskussion im Zusammenhang mit der Verfassung des Abschlußberichtes Projektbereich D, FZW Göttingen

Güßefeldt, J. (1997): Grundsätzliche Überlegungen zu Regrionalisierungsmodellen; Geographische Zeitschrift, 45. Jg. Heft 1: 1-19, Franz Steiner Verlag Wiesbaden GmbH, Sitz Stuttgart

Häckel, H. (1990): Meteorologie; Häckel, Hans. -2., verb. Auflage. - Stuttgart: Ulmer, 1990 (UTB für Wissenschaft: Uni-Taschenbücher; 1338)

Hartmann, F.-K. und F. Schnelle (1970): Klimagrundlagen natürlicher Waldstufen und ihrer Waldgesellschaften in deutschen Mittelgebirgen; Stuttgart: G. Fischer

Hartung, J. (1995): Lehr- und Handbuch der angewandten Statistik; Joachim Hartung; Baerbel Elpelt; Karl-Heinz Kloesener. - 10., durchges. Aufl. München [u. a.]: Oldenbourg

Heinrich, U. (1994): Flächenhafte Ableitung der Klimaparameter Niederschlag und Temperatur mittels geostatistischer Verfahren. In: Schröder, W. et al. (1994): 
Neuere statischtische Verfahren und Modellbildung in der Geoökologie. Vieweg Verlag

Ibrom, A. (1993): Die Deposition und die Pflanzenauswaschung (Leaching) von Pflanzennährstoffen in einem Fichtenbestand im Solling. Bericht d. Forschungszentrums Waldökosysteme d. Univ. Göttingen, Reihe A 106

Isermann, K. und R. Isermann (1999): Emissionen und Immissionen von Ammoniak und Stickoxiden. AFZ/Der Wald 6: 302-309

Jansen, M., M. Schroetter, C. Eberl, K.-J. Meiwes, B. Ulrich und B. Sloboda (1994): Prototyp eines wissensbasierten Systems zur Planung von Kalkungsmaßnahmen im Westharz. Dt. Verb. Forstlicher Forschungsanstalten, Sek. Forstl. Biom. u. Informatik, Tagungsband Ljubljana, Sept. 1994: 122-136

Jansen, M., J. Saborowski, R. Schulz und K. Radler (1997): Spatial Variation of Temperature and Precipitation in the Harz Mountains; Intern. Scientific Conference: Forest-Wood-Environment 97, Zvolen/Slowakei 8.-11.9.1997

Jansen, M., J. Saborowski, M. Judas u. A.W. Bitter (in Vorb.): Spatial modelling in forest ecology and management. Springer Verlag, Heidelberg Berlin

Jensen, H. (1986): Regionalisierung der Verteilungsfunktion des jährlichen Maximums des Tagesniederschlages im Kanton Zürich; Zürcher Geographische Schriften, ETH. Eidgenössische Technische Hochschule Zürich

Journel, A.G. und C.J. Huijbregts (1978): Mining Geostatistics. Academic Press, London

König, mdl. Mitteilung (1997): Auskunft zur Analyse von Depositionsproben. Niedersächsische Forstliche Versuchsanstalt Göttingen; 22.4.1997

Konitzer, mdl. Mitteilung (1998): Erkenntnisse im Rahmen des Forschungsvorhabens „FIS-Ö und Ökonomische Bewertung“ des Forschungszentrums Waldökosysteme 
Klein, G. (1994): Regionalisierung von Niederschlag mit Hilfe digitaler Geländeinformationen. Freiburger Geographische Hefte, Herausgegeben von Hermann Goßmann, Heft 44

Kumm, H. (1983): Vergleichsmessungen mit windgeschützten und ungeschützten Niederschlagsmeßgeräten; Berichte des Deutschen Wetterdienstes Nr. 162; Im Selbstverlag des Deutschen Wetterdienstes, Offenbach a. Main 1983

Kurtyka, J.C. (1953): Precipitation measurements bibliography; partially annotated. Annual Rep., 20, 178 p., State Water Surv., Dept. of Registr. and Education, Urbana

Laughlin, G.P. und J.D. Kalma (1990): Frost Risk Mapping for Landscape Planning: A Methodology; Theoretical and Applied Climatology 42: 41-51; SpringerVerlag

Lenz, R., R. Stary und S. Mendler (1996): Regionalspezifische Depositionsalgorithmen auf mittlerer Maßstabsebene (1:50000); Abschlußbericht des F\&E Vorhabens mit der Nummer 2-508514; Auftraggeber: Bundesanstalt für Geowissenschaften und Rohstoffe (BGR), Hannover

Lindkvist, L und S. Lindqvist (1997): Spatial and temporal variability of nocturnal summer frost in elevated complex terrain, Agricultural and Forest Meteorology 87: 139-159

Matheron, G. (1963): Principles of geostatisctics; Economic Geology, 58: 1246-1266

Matzner, E. (1988): Der Stoffumsatz zweier Waldökosysteme im Solling. Bericht d Forschungszentrums Waldökosysteme d. Univ. Göttingen, Reihe A 40

Meesenburg, H., K.J. Meiwes und R. Schultz-Sternberg (1994): Entwicklung der atmogenen Stoffeinträge in niedersächsische Waldbestände; Forst und Holz 49 Jg. Nr. 9: 236-238

Meyer, M. (1992): Untersuchungen zur Restabilisierung geschaedigter Waldökosysteme im norddeutschen Küstenraum (Fallstudie Wingst II). Berichte d. Forschungszentrums Waldökosysteme d. Univ. Göttingen, Reihe A 94 
NLÖ (1992): Bericht über die Untersuchung von Niederschlagswasser in Niedersachsen 1988 bis 1990; Niedersächsisches Landesamt für Ökologie, Hildesheim

NLÖ (1993): Depositions-Meßnetz Niedersachsen, Belastung von Wasser und Boden durch Schadstoffe in Luft und Niederschlägen; Niedersächsisches Landesamt für Ökologie, Hildesheim

Pebesma, E. (1997): GSTAT User's Manual. Dept. of Physical Geography, Utrecht University

Rodda; J.C. (1971): The precipitation measurement paradox - the instrument accuracy problem. Geneva: World Meteorol. Organiz.

Ripley, B.D. (1981): Spatial Statistics, Wiley, New York

Saborowski, J.und J. Stock (1994): Die Regionalisierung von Niederschlagsdaten im Harz, AFJZ: 165 (7): 117-122

Saborowski, J. mdl. Mitteilung (1996): Mdl. Mitteilung zur Frage des notwendigen Stichprobenumfangs zur gestatistischen Analyse

Saborowski, J. mdl. Mitteilung (1998): Mdl. Mitteilung nach der Überprüfung der Fragestellung an dem in Kap. 4.1.2.1 beschriebenen TRANSEKTDatenkollektiv

Sah, S.P. (1990): Vergleich des Stoffhaushaltes zweier Buchenwaldökosysteme auf Kalkgestein und auf Buntsandstein. Dissertation zur Erlangung des Doktorgrades des Forstwissenschaftlichen Fachbereiches der Georg-AugustUniversität Göttingen; Berichte d. Forschungszentrums Waldökosysteme Göttingen d. Univ. Göttingen, Reihe A 59

Schober, R. (1987): Ertragstafeln wichtiger Baumarten; neubearbeitete Auflage, J.D. Sauerländer's Verlag. Frankfurt a. M.

Schröder, W., L. Vetter und O. Fränzle (Hrsg.) (1994): Neuere statischtische Verfahren und Modellbildung in der Geoökologie. Vieweg Verlag 
Schultz, R. (1987): Vergleichende Betrachtung des Schwermetallhaushalts verschiedener Waldökosysteme Norddeutschlands. Berichte d. Forschungszentrums Waldökosysteme d. Univ. Göttingen, Reihe A 32

Schulz (in Vorb.): Spatial indicators of relief, climate, soil and macrofauna. In: Jansen. M, J. Saborowski, M. Judas u. A.W. Bitter (in Vorb.): Spatial modelling in forest ecology and management. Springer Verlag, Heidelberg Berlin

Steiner, Ch. und M.J. Lexer (1998): Ein klimasensitives statisches Modell zur Beurteilung der Baumarteneignung; Forstarchiv 69, p. 92-103

Stock, R. und B. Sloboda (1991): Einsatzmöglichkeiten eines flächenbezogenen Informationssystems im forstökologischen Management und Monitoring Forsttechnische Aspekte. In: Sloboda, B. und S. Smelko: Biometrische Beiträge $\mathrm{zu}$ statischen und dynamischen Modellansätzen in den Forstwissenschaften und der Praxis. Dt. Verb. Forstl. Forschungsanstalten, Sek. Forstl. Biom. u. Inform., 5.Tag. Martin/Zvolen

Tobias, K., R. Lang, R. Lenz und P. Schall (1989): Flächenbezogene Abschätzungen der Depositionsmengen von Protonen, Stickstoff, Calcium und Magnesium in vier Schwerpunktforschungsräumen der Bundesrepublik Deutschland, GIS $4 / 89$

UBA (1993): Zusammenfassung der Forschungsergebnisse zur Waldschadensproblematik in den neuen Ländern (Band 2: Eberswalde); Umweltbundesamt, Forschungsberichte 10803 046/60, UBA- FB 92-137; 10803 046/61, UBA- FB 92-062 und 10803 046/62, UBA- FB 92-122; Texte $2 / 93$.

Ulrich, B. (1983): Abiotische Folgewirkungen der weiträumigen Ausbreitung von Luftverunreinigung; Bernhard Ulrich; Egbert Matzner. Umweltforschungsplan d. Bundesministers des Innern. Luftreinhaltung Forschungsbericht 10402615. 
Ulrich, B. (1991): Rechenweg zur Schätzung der Flüsse in Waldökosystemen; Identifizierung der sie bedingenden Prozesse. Berichte d. Forschungszentrums Waldökosysteme d. Univ. Göttingen, Reihe B 24

Vent-Schmidt, V. (1985): A regression model for the calculation of the spatial distribution of mean air temperatures using an orographic data base. Meteorologische Rundschau 38 (6): 185-187

VDI (1987): Acidic Precipitation. Formation and Impact on Terrestrial Ecosystems. Kommission Reinhaltung der Luft, Düsseldorf

Wiedemann (1936/42): Die Fichte. Mitteilung aus Forstwirtschaft und Forstwissenschaft 1936 / Eine Korrektur a. d. Fichtenertragstafel; Mitteilung aus Forstwirtschaft und Forstwissenschaft 1942; aus: SCHOBER 1987

Wiedey, E.A. (1987): Raten der Deposition, Akkumulation und des Austrags toxischer Luftverunreinigungen als Maß der Belastung und Belastbarkeit von Waldökosystemen. - In: Exkursionsführer Harz 1987: Harzexkursion des Forschungsbeirats Waldschäden, 3./4. Juli 1987, unveröffentlicht

Winkler, P. (1983): Der Säuregehalt von Aerosol, Nebel und Niederschlägen. VDIBerichte 500

WMO (1972): World Meteorological Organization : Geilo (Norway) Symposium on orographic influence on the distribution of precipitation, WMO-NO. 326, Geneva

Xijun Xu und K. Blanck (1994): Räumliche Variabilität der Kronentraufelösung auf Solling-Fichtenflächen. In: AG-Deposition der Ökosystemforschungszentren (1994)

Zöttl, H.W., H.-P. Ende, R.F. Hüttl und J.C. Liu (1990): Auswirkungen diagnostischer Düngung in geschädigten Waldbeständen Südwestdeutschlands und Nordamerikas; Abschlußbericht BMFT-Forschungsvorhaben Nr. 0339182B 


\section{Anhang}

Anhang 1: Werte der Interzeptionsindices für Fichte / Nadelbäume und der originären Parameter aus der Fichtenertragstafel der 2. Ertragsklasse nach WIEDEMANN (1936/42)

\begin{tabular}{cccccc}
\hline $\begin{array}{c}\text { Alter } \\
\text { [Jahre] }\end{array}$ & $\begin{array}{c}\text { Mittelhöhe } \\
\text { [m] }\end{array}$ & $\begin{array}{c}\text { Derbholzformzahl } \\
\mathbf{0 , 9 . . .}\end{array}$ & Index 1 & Index 2 & Index 3 \\
\hline 20 & 5,1 & 121 & 23,73 & 53,58 & 80,52 \\
25 & 6,7 & 281 & 41,94 & 108,56 & 174,66 \\
30 & 8,6 & 368 & 42,79 & 125,49 & 214,89 \\
35 & 10,7 & 422 & 39,44 & 129,01 & 233,33 \\
40 & 12,8 & 466 & 36,41 & 130,25 & 246,37 \\
45 & 14,9 & 498 & 33,42 & 129,01 & 253,47 \\
50 & 16,9 & 510 & 30,18 & 124,06 & 251,54 \\
55 & 18,8 & 513 & 27,29 & 118,31 & 246,36 \\
60 & 20,5 & 514 & 25,07 & 113,52 & 241,56 \\
65 & 22,0 & 512 & 23,27 & 109,16 & 236,41 \\
70 & 23,3 & 508 & 21,80 & 105,24 & 231,22 \\
75 & 24,5 & 502 & 20,49 & 101,42 & 225,64 \\
80 & 25,6 & 495 & 19,34 & 97,83 & 220,06 \\
85 & 26,6 & 489 & 18,38 & 94,81 & 215,32 \\
90 & 27,6 & 482 & 17,46 & 91,75 & 210,29 \\
95 & 28,5 & 476 & 16,70 & 89,16 & 206,01 \\
100 & 29,3 & 472 & 16,11 & 87,20 & 202,87 \\
105 & 31,1 & 468 & 15,05 & 83,92 & 198,18 \\
110 & 30,8 & 465 & 15,10 & 83,79 & 197,39 \\
115 & 31,5 & 461 & 14,63 & 82,14 & 194,59 \\
120 & 32,1 & 459 & 14,30 & 81,01 & 192,84 \\
\hline
\end{tabular}


Anhang 2: Werte der Interzeptionsindices für Buche / Laubbaumarten und der originären Parameter aus der Buchenertragstafel der 2. Ertragsklasse nach SCHOBER (1967)

\begin{tabular}{cccccc}
\hline $\begin{array}{c}\text { Alter } \\
\text { [Jahre] }\end{array}$ & $\begin{array}{c}\text { Mittelhöhe } \\
{[\mathbf{m}]}\end{array}$ & $\begin{array}{c}\text { Derbholzformzahl } \\
\mathbf{0 , 9 . .}\end{array}$ & Index 1 & Index 2 & Index 3 \\
\hline 30 & 6,7 & & & & \\
35 & 8,5 & 234 & 27,53 & 80,26 & 137,04 \\
40 & 10,5 & 318 & 30,29 & 98,14 & 176,66 \\
45 & 12,6 & 374 & 29,68 & 105,36 & 198,51 \\
50 & 14,5 & 409 & 28,21 & 107,41 & 209,60 \\
55 & 16,3 & 432 & 26,50 & 107,00 & 215,00 \\
60 & 17,9 & 450 & 25,14 & 106,36 & 218,78 \\
65 & 19,4 & 460 & 23,71 & 104,44 & 219,18 \\
70 & 20,7 & 469 & 22,66 & 103,08 & 219,88 \\
75 & 22,0 & 474 & 21,55 & 101,06 & 218,86 \\
80 & 23,2 & 478 & 20,60 & 99,24 & 217,80 \\
85 & 24,3 & 482 & 19,84 & 97,78 & 217,09 \\
90 & 25,4 & 485 & 19,09 & 96,23 & 216,04 \\
95 & 26,4 & 489 & 18,52 & 95,17 & 215,73 \\
100 & 27,4 & 491 & 17,92 & 93,80 & 214,61 \\
105 & 28,2 & 494 & 17,52 & 93,03 & 214,37 \\
110 & 29,0 & 496 & 17,10 & 92,10 & 213,74 \\
115 & 29,8 & 497 & 16,68 & 91,04 & 212,72 \\
120 & 30,5 & 498 & 16,33 & 90,17 & 211,91 \\
125 & 31,1 & 499 & 16,05 & 89,48 & 211,31 \\
130 & 31,7 & 500 & 15,77 & 88,81 & 210,72 \\
135 & 32,2 & 501 & 15,56 & 88,29 & 210,32 \\
140 & 32,7 & 502 & 15,35 & 87,79 & 209,93 \\
145 & 33,2 & 502 & 15,12 & 87,12 & 209,13 \\
150 & 33,7 & 503 & 14,93 & 86,65 & 208,77 \\
\hline & & & & & \\
& & & &
\end{tabular}

Anhang 3: Zur Regionalisierung von Bulk-Depositionen verwendete Meßstationen; Waldstationen sind grau unterlegt

\begin{tabular}{lcccccccc}
\hline \multicolumn{1}{c}{ Standortname } & Rechtsw. & Hochw. & SO $_{4}$-S & NO $_{3}$-N & NH $_{4}$-N & Cl & pH & Quelle \\
\hline Göttinger Wald & 3572700 & 5710380 & $\mathrm{x}$ & $\mathrm{x}$ & $\mathrm{x}$ & $\mathrm{x}$ & $\mathrm{x}$ & FZW \\
Heide/Eiche (Sellhorn 66b1) & 3560150 & 5894800 & $\mathrm{x}$ & $\mathrm{x}$ & $\mathrm{x}$ & $\mathrm{x}$ & $\mathrm{x}$ & FZW \\
Heide/Kiefer (Sellhorn 153) & 3559200 & 5893200 & $\mathrm{x}$ & $\mathrm{x}$ & $\mathrm{x}$ & $\mathrm{x}$ & $\mathrm{x}$ & FZW \\
Solling B1-Fläche & 3538950 & 5736530 & $\mathrm{x}$ & $\mathrm{x}$ & $\mathrm{x}$ & $\mathrm{x}$ & $\mathrm{x}$ & FZW \\
Solling F1-Fläche & 3540200 & 5736720 & $\mathrm{x}$ & $\mathrm{x}$ & $\mathrm{x}$ & $\mathrm{x}$ & $\mathrm{x}$ & FZW \\
Wingst Abt. 221 & 3504950 & 5954100 & $\mathrm{x}$ & $\mathrm{x}$ & $\mathrm{x}$ & $\mathrm{x}$ & $\mathrm{x}$ & FZW \\
Wingst Abt. 28 & 3506050 & 5947450 & $\mathrm{x}$ & $\mathrm{x}$ & $\mathrm{x}$ & $\mathrm{x}$ & $\mathrm{x}$ & FZW \\
Göttinger Wald Freifläche & 3567750 & 5717150 & $\mathrm{x}$ & $\mathrm{x}$ & $\mathrm{x}$ & $\mathrm{x}$ & $\mathrm{x}$ & FZW \\
Heide Freifläche & 3559100 & 5894200 & $\mathrm{x}$ & $\mathrm{x}$ & $\mathrm{x}$ & $\mathrm{x}$ & $\mathrm{x}$ & FZW \\
Solling B1-Freifläche & 3539750 & 5736630 & $\mathrm{x}$ & $\mathrm{x}$ & $\mathrm{x}$ & $\mathrm{x}$ & $\mathrm{x}$ & FZW \\
Wingst Abt. 28 Freifläche & 3505750 & 5947450 & $\mathrm{x}$ & $\mathrm{x}$ & $\mathrm{x}$ & $\mathrm{x}$ & $\mathrm{x}$ & FZW \\
BGSW Braunschweig/Broitzem & 3600750 & 5789170 & $\mathrm{x}$ & $\mathrm{x}$ & & $\mathrm{x}$ & & LÜN \\
ENNW Emden/Twixlum & 3375725 & 5917018 & $\mathrm{x}$ & $\mathrm{x}$ & & $\mathrm{x}$ & & LÜN \\
HRNW Hannover/Vinnhorst & 3547820 & 5809200 & $\mathrm{x}$ & $\mathrm{x}$ & & $\mathrm{x}$ & & LÜN \\
HRSW Hannover/Linden & 3548750 & 5803080 & $\mathrm{x}$ & $\mathrm{x}$ & & $\mathrm{x}$ & & LÜN \\
NMCC Nordenham/City & 3465250 & 5929330 & $\mathrm{x}$ & $\mathrm{x}$ & & $\mathrm{x}$ & & LÜN \\
OGCC Oker & 3602080 & 5752810 & $\mathrm{x}$ & $\mathrm{x}$ & & $\mathrm{x}$ & & LÜN \\
OGNO Harlingerode & 3604503 & 5753653 & $\mathrm{x}$ & $\mathrm{x}$ & & $\mathrm{x}$ & & LÜN \\
\hline
\end{tabular}




\begin{tabular}{|c|c|c|c|c|c|c|c|c|}
\hline PECC Peine/llsede & 3585050 & 5799660 & $x$ & $x$ & & $x$ & & LÜN \\
\hline PESSP eine/Ölsburg & 3582570 & 5793470 & $x$ & $x$ & & $x$ & & LÜN \\
\hline WLCC Wolfenbüttel & 3605637 & 5781464 & $x$ & $x$ & & $x$ & & LÜN \\
\hline BGNW Braunschweig/Sackring & 3601798 & 5793676 & $x$ & $x$ & & $x$ & & LÜN \\
\hline $\begin{array}{l}\text { BGOO } \\
\text { Braunschweig/Nehrkornweg }\end{array}$ & 3607856 & 5794087 & $x$ & $x$ & & $x$ & & LÜN \\
\hline HROO Hannover/Misburg & 3558460 & 5806680 & $x$ & $x$ & & $x$ & & LÜN \\
\hline OGNN Oker/Mühlenstrasse & 3602200 & 5754470 & $x$ & $x$ & & $x$ & & LÜN \\
\hline OGNW Oker/Im Schleeke & 3600480 & 5754160 & $x$ & $x$ & & $x$ & & LÜN \\
\hline OGOO Bad Harzburg/Kurpark & 3607412 & 5749630 & $x$ & $x$ & $x$ & $x$ & $x$ & LÜN \\
\hline Buer- Ostenwalde M.55 & 3455448 & 5791577 & $x$ & $x$ & $x$ & $x$ & $x$ & NLÖ \\
\hline Riefensbeck & 3599885 & 5738485 & $x$ & & $x$ & $x$ & $x$ & NLÖ \\
\hline Seesen & 3585330 & 5753720 & $x$ & $x$ & $x$ & $x$ & $x$ & NLÖ \\
\hline Bad Rothenfelde & 3443600 & 5775800 & $x$ & $x$ & $x$ & $x$ & $x$ & NLÖ \\
\hline Doerenberg Hanglage & 3433400 & 5783400 & $x$ & $x$ & $x$ & $x$ & $x$ & NLÖ \\
\hline Doerenberg Kuppe & 3434000 & 5783500 & $x$ & $x$ & $x$ & $x$ & $x$ & NLÖ \\
\hline Osnabrück Zentrum & 3432700 & 5794100 & $x$ & $x$ & $x$ & $x$ & $x$ & NLÖ \\
\hline Damme-Harlinghausen & 3440800 & 5820100 & $x$ & $x$ & $x$ & $x$ & $x$ & NLÖ \\
\hline Triller Berg & 3421300 & 5819300 & $x$ & $x$ & $x$ & $x$ & $x$ & NLÖ \\
\hline Kreuzberg & 3412700 & 5833600 & $x$ & $x$ & $x$ & $x$ & $x$ & NLÖ \\
\hline Lingen-Baccum & 3389827 & 5817844 & $x$ & $x$ & $x$ & $x$ & $x$ & NLÖ \\
\hline Bad Bentheim & 3377482 & 5795335 & $x$ & $x$ & $x$ & $x$ & $x$ & NLÖ \\
\hline Neuenhaus- Itterberg & 3353058 & 5819472 & $x$ & $x$ & $x$ & $x$ & $x$ & NLÖ \\
\hline Rütenbrock & 3373647 & 5857155 & $\mathrm{x}$ & $x$ & $x$ & $x$ & $x$ & NLÖ \\
\hline Edewecht & 3440000 & 5888300 & $x$ & $x$ & $x$ & $x$ & $x$ & NLÖ \\
\hline Hude-Hasbruch & 3464800 & 5882400 & $x$ & $x$ & & $x$ & $x$ & NLÖ \\
\hline Ovelgönne-Colmar & 3457800 & 5912700 & $x$ & $x$ & $x$ & $x$ & $x$ & NLÖ \\
\hline Friedeburg & 3421000 & 5923200 & $x$ & $x$ & $x$ & $x$ & $x$ & NLÖ \\
\hline Emden-Knock & 3368701 & 5913519 & $x$ & $x$ & $x$ & $x$ & $x$ & NLÖ \\
\hline Ahlhorn & 3443100 & 5865900 & $x$ & $x$ & $x$ & $x$ & $x$ & NLÖ \\
\hline Bückeberge & 3513200 & 5792400 & $x$ & $x$ & $x$ & $x$ & $x$ & NLÖ \\
\hline Schwaförden & 3491800 & 5845200 & $x$ & $x$ & $x$ & $x$ & $x$ & NLÖ \\
\hline Brockel & 3536500 & 5882600 & $x$ & $x$ & $x$ & $x$ & $x$ & NLÖ \\
\hline Osterholz-Scharmbeck & 3488800 & 5913800 & $x$ & $x$ & $x$ & $x$ & $x$ & NLÖ \\
\hline Stade-Mulsum & 3518700 & 5933000 & $x$ & $x$ & $x$ & $x$ & $x$ & NLÖ \\
\hline Drangstedt & 3480300 & 5943600 & $x$ & $x$ & $x$ & $x$ & $x$ & NLÖ \\
\hline Stuvenwald & 3555100 & 5917200 & $x$ & $x$ & $x$ & $x$ & $x$ & NLÖ \\
\hline Scharnebeck & 3603348 & 5906940 & $x$ & $x$ & $x$ & $x$ & $x$ & NLÖ \\
\hline Siemen & 3647701 & 5880471 & $x$ & $x$ & $x$ & $x$ & $x$ & NLÖ \\
\hline Knesebeck & 3617198 & 5839056 & $x$ & $x$ & $x$ & $x$ & $x$ & NLÖ \\
\hline Unterlüß & 3588000 & 5856100 & $x$ & $x$ & $x$ & $x$ & $x$ & NLÖ \\
\hline Berkhof & 3552300 & 5831100 & $x$ & $x$ & $x$ & $x$ & $x$ & NLÖ \\
\hline Norderney Stadt & 3378430 & 5954351 & $x$ & $x$ & $x$ & $x$ & $x$ & NLÖ \\
\hline Norderney Flughafen & 3382930 & 5954261 & $x$ & $x$ & $x$ & $x$ & $x$ & NLÖ \\
\hline Holzminden-Schießaus & 3540100 & 5743100 & $x$ & $x$ & $x$ & $x$ & $x$ & NLÖ \\
\hline Hann.-Münden-Hemeln & 3544500 & 5706500 & $x$ & $x$ & $x$ & $x$ & $x$ & NLÖ \\
\hline Northeim-Westerhof & 3575600 & 5734200 & $\mathrm{x}$ & $x$ & $x$ & $\mathrm{x}$ & $x$ & NLÖ \\
\hline Riefensbeek (Freiland) & 3600000 & 5738400 & $x$ & $x$ & $x$ & $x$ & $x$ & NLÖ \\
\hline Seesen-Hohestein & 3585300 & 5753600 & $x$ & $x$ & $x$ & $x$ & $x$ & NLÖ \\
\hline
\end{tabular}




\begin{tabular}{lcccccccc}
\hline Diekholzen-Petze & 3563600 & 5770800 & $\mathrm{x}$ & $\mathrm{x}$ & $\mathrm{x}$ & $\mathrm{x}$ & $\mathrm{x}$ & $\mathrm{NLÖ}$ \\
Königslutter & 3622508 & 5788328 & $\mathrm{x}$ & $\mathrm{x}$ & $\mathrm{x}$ & $\mathrm{x}$ & $\mathrm{x}$ & $\mathrm{NLÖ}$ \\
Buer-Ostenwalde Freiland & 3455500 & 5791400 & $\mathrm{x}$ & $\mathrm{x}$ & $\mathrm{x}$ & $\mathrm{x}$ & $\mathrm{x}$ & $\mathrm{NLÖ}$ \\
\hline
\end{tabular}

Anhang 4: Literaturangaben zu den vom FZW verwendeten Meßstellen

\begin{tabular}{ll}
\hline Standortname & Literatur \\
\hline Göttinger Wald & Sah (1990) \\
Göttinger Wald Freifläche & Bredemeier (1987) \\
Heide Freifläche & Schultz (1987) \\
Heide/Eiche (Sellhorn 66b1) & Schultz (1987) \\
Heide/Kiefer (Sellhorn 153) & Schultz (1987) \\
Solling B1-Fläche & Sah (1990), Ellenberg et al (1986), Matzner (1988), \\
Solling B1-Freifläche & Ellenberg et al (1986), Matzner (1988), \\
Solling F1-Fläche & Ellenberg et al (1986), Matzner (1988), \\
Wingst Abt. 221 & Meyer (1992), Büttner (1986) \\
Wingst Abt. 28 & Meyer (1992), Büttner (1986) \\
Wingst Abt. 28 Freifläche & Meyer (1992), Büttner (1986) \\
\hline
\end{tabular}

Anhang 5: Gegenüberstellung von Verteilungen räumlicher Variablen (deskriptive Statistiken) für das niedersächsische Bergland; NitratStationen

\begin{tabular}{|c|c|c|c|c|c|c|c|c|}
\hline \multicolumn{4}{|c|}{$\begin{array}{c}\text { Bergland } \\
\text { (Rasterwerte; Rasterweite: } \mathbf{5 0} \mathbf{~ m} \text { ) }\end{array}$} & \multirow[t]{2}{*}{ Variable } & \multicolumn{4}{|c|}{$\begin{array}{c}\text { Nitrat-Stationen; } \mathbf{n = 1 9} \\
\text { (Repräsentanzmängel in \%) }\end{array}$} \\
\hline $\min$ & $\max$ & mean & std. dev. & & $\min$ & $\max$ & mean & std. dev. \\
\hline 35 & 953 & 218,23 & 130,75 & $\begin{array}{l}\text { Geländehöhe } \\
\text { [m ü. NN] }\end{array}$ & $\begin{array}{r}73 \\
(2,5)\end{array}$ & $\begin{array}{r}754 \\
(0,6)\end{array}$ & 311,3 & 150,0 \\
\hline 0 & 136 & 8,34 & 8,97 & $\begin{array}{c}\text { Hangneigung } \\
{[\%]}\end{array}$ & $\begin{array}{r}0 \\
(0)\end{array}$ & $\begin{array}{r}26 \\
(5,1)\end{array}$ & 9,0 & 6,9 \\
\hline 0 & 368 & 107,61 & 33,91 & $\begin{array}{c}\text { Insolation Jan. } \\
\text { [ ] }\end{array}$ & $\begin{array}{r}63 \\
(7,8)\end{array}$ & $\begin{array}{r}203 \\
(1,3)\end{array}$ & 111,1 & 37,0 \\
\hline 0 & 36 & 33,25 & 5,50 & $\begin{array}{l}\text { Rel. Exponiertheit } \\
\text { (0 bis 36) }\end{array}$ & $\begin{array}{r}13 \\
(1,2)\end{array}$ & $\begin{array}{l}36 \\
(0)\end{array}$ & 32,2 & 6,6 \\
\hline 0 & 2000 & 92,25 & 311,76 & $\begin{array}{l}\text { Kaltluftabfluß } \\
(0 \text { bis } 2000)\end{array}$ & $\begin{array}{r}0 \\
(0)\end{array}$ & $\begin{array}{l}1529 \\
(2,0)\end{array}$ & 123,0 & 363,9 \\
\hline 0 & 1096 & 110,10 & 110,67 & $\begin{array}{l}\text { Leewirbel } \\
{[\mathrm{m}]}\end{array}$ & $\begin{array}{r}0 \\
(0)\end{array}$ & $\begin{array}{r}609 \\
(0,3)\end{array}$ & 120,6 & 169,0 \\
\hline
\end{tabular}

Anhang 6: Gegenüberstellung von Verteilungen räumlicher Variablen (deskriptive Statistiken) für das niedersächsische Bergland; Ammonium-Stationen

\begin{tabular}{|c|c|c|c|c|c|c|c|c|}
\hline \multicolumn{4}{|c|}{$\begin{array}{c}\text { Bergland } \\
\text { (Rasterwerte; Rasterweite: } \mathbf{5 0} \mathbf{~ m} \text { ) }\end{array}$} & \multirow[t]{2}{*}{ Variable } & \multicolumn{4}{|c|}{$\begin{array}{c}\text { Ammonium-Stationen; } \mathbf{n = 1 4} \\
\text { (Repräsentanzmängel in \%) }\end{array}$} \\
\hline $\min$ & $\max$ & mean & std. dev. & & Min & $\max$ & mean & std. dev. \\
\hline 35 & 953 & 218,23 & 130,75 & $\begin{array}{l}\text { Geländehöhe } \\
\text { [m ü. NN] }\end{array}$ & $\begin{array}{r}73 \\
(2,5)\end{array}$ & $\begin{array}{r}754 \\
(0,6)\end{array}$ & 341,6 & 163,9 \\
\hline 0 & 136 & 8,34 & 8,97 & $\begin{array}{c}\text { Hangneigung } \\
{[\%]}\end{array}$ & $\begin{array}{r}1 \\
(7,8)\end{array}$ & $\begin{array}{r}26 \\
(5,1)\end{array}$ & 10,2 & 7,1 \\
\hline 0 & 368 & 107,61 & 33,91 & $\begin{array}{c}\text { Insolation Januar } \\
\text { [ ] }\end{array}$ & $\begin{array}{r}63 \\
(7,8) \\
\end{array}$ & $\begin{array}{r}203 \\
(1,3)\end{array}$ & 117,8 & 40,7 \\
\hline 0 & 36 & 33,25 & 5,50 & $\begin{array}{c}\text { Relative Exponiertheit } \\
\text { (0 bis } 36)\end{array}$ & $\begin{array}{r}17 \\
(2,2)\end{array}$ & $\begin{array}{l}36 \\
(0)\end{array}$ & 33,3 & 5,3 \\
\hline 0 & 2000 & 92,25 & 311,76 & $\begin{array}{c}\text { Kaltluftabfluß } \\
(0 \text { bis } 2000)\end{array}$ & $\begin{array}{r}0 \\
(0) \\
\end{array}$ & $\begin{array}{r}37 \\
(21,8) \\
\end{array}$ & 9,8 & 10,4 \\
\hline 0 & 1096 & 110,10 & 110,67 & $\begin{array}{l}\text { Leewirbel } \\
{[\mathrm{m}]}\end{array}$ & $\begin{array}{r}0 \\
(0)\end{array}$ & $\begin{array}{r}172 \\
(22,9)\end{array}$ & 42,8 & 53,4 \\
\hline
\end{tabular}


Anhang 7: Gegenüberstellung von Verteilungen räumlicher Variablen (deskriptive Statistiken) für das niedersächsische Tiefland; NitratStationen

\begin{tabular}{|c|c|c|c|c|c|c|c|c|}
\hline \multicolumn{4}{|c|}{$\begin{array}{c}\text { Tiefland } \\
\text { (Rasterwerte; Rasterweite: } \mathbf{5 0} \mathbf{~ m})\end{array}$} & \multirow[t]{2}{*}{ Variable } & \multicolumn{4}{|c|}{$\begin{array}{c}\text { Nitrat-Stationen; } \mathbf{n}=40 \\
\text { (Repräsentanzmängel in \%) }\end{array}$} \\
\hline $\min$ & $\max$ & mean & std. dev. & & $\min$ & $\max$ & mean & std. dev. \\
\hline-11 & 172 & 35,53 & 28,35 & $\begin{array}{l}\text { Geländehöhe } \\
\text { [m ü. NN] }\end{array}$ & $\begin{array}{r}0 \\
(0,4)\end{array}$ & $\begin{array}{r}114 \\
(0,4)\end{array}$ & 47,2 & 35,4 \\
\hline 0 & 63 & 0,68 & 1,34 & $\begin{array}{c}\text { Hangneigung } \\
{[\%]}\end{array}$ & $\begin{array}{r}0 \\
()\end{array}$ & $\begin{array}{r}13 \\
(0,1)\end{array}$ & 1,5 & 2,4 \\
\hline 0 & 306 & 108,95 & 5,06 & $\begin{array}{c}\text { Insolation Jan. } \\
\text { [ ] }\end{array}$ & $\begin{array}{r}102 \\
(4,5)\end{array}$ & $\begin{array}{r}137 \\
0,2\end{array}$ & 111,2 & 5,4 \\
\hline 0 & 36 & 35,99 & 0,28 & $\begin{array}{l}\text { Rel. Exponiertheit } \\
\text { (0 bis 36) }\end{array}$ & $\begin{array}{r}31 \\
(0,1)\end{array}$ & $\begin{array}{l}36 \\
()\end{array}$ & 35,9 & 0,8 \\
\hline 0 & $\begin{array}{r}200 \\
0\end{array}$ & 138,01 & 398,09 & $\begin{array}{l}\text { Kaltluftabfluß } \\
(0 \text { bis } 2000)\end{array}$ & $\begin{array}{r}1 \\
(4,4)\end{array}$ & $\begin{array}{r}2000 \\
()\end{array}$ & 186,5 & 506,3 \\
\hline 0 & 406 & 12,04 & 18,74 & $\begin{array}{l}\text { Leewirbel } \\
{[\mathrm{m}]}\end{array}$ & $\begin{aligned} 0 \\
()\end{aligned}$ & $\begin{array}{r}84 \\
(1,2)\end{array}$ & 14,5 & 19,4 \\
\hline
\end{tabular}

Anhang 8: Gegenüberstellung von Verteilungen räumlicher Variablen (deskriptive Statistiken) für das niedersächsische Tiefland; Ammonium-Stationen

\begin{tabular}{|c|c|c|c|c|c|c|c|c|}
\hline \multicolumn{4}{|c|}{$\begin{array}{c}\text { Tiefland } \\
\text { (Rasterwerte; Rasterweite: } \mathbf{5 0} \mathbf{~ m} \text { ) }\end{array}$} & \multirow[t]{2}{*}{ Variable } & \multicolumn{4}{|c|}{$\begin{array}{c}\text { Ammonium-Stationen; } \mathbf{n}=28 \\
\text { (Repräsentanzmängel in \%) }\end{array}$} \\
\hline $\min$ & $\max$ & mean & std. dev. & & $\min$ & $\max$ & mean & std. dev. \\
\hline-11 & 172 & 35,53 & 28,35 & $\begin{array}{l}\text { Geländehöhe } \\
\text { [m ü. NN] }\end{array}$ & $\begin{array}{r}0 \\
(0,4)\end{array}$ & $\begin{array}{r}114 \\
(0,4)\end{array}$ & 43,4 & 37,1 \\
\hline 0 & 63 & 0,68 & 1,34 & $\begin{array}{c}\text { Hangneigung } \\
{[\%]}\end{array}$ & $\begin{array}{r}0 \\
(0)\end{array}$ & $\begin{array}{r}13 \\
(0,1)\end{array}$ & 1,6 & 2,5 \\
\hline 0 & 306 & 108,95 & 5,06 & $\begin{array}{c}\text { Insolation Januar } \\
\text { [ ] }\end{array}$ & $\begin{array}{r}102 \\
(4,5)\end{array}$ & $\begin{array}{r}120 \\
(2,1)\end{array}$ & 110,7 & 3,5 \\
\hline 0 & 36 & 35,99 & 0,28 & $\begin{array}{l}\text { Relative Exponiertheit } \\
\text { ( } 0 \text { bis } 36)\end{array}$ & $\begin{array}{r}31 \\
(0,1)\end{array}$ & $\begin{array}{l}36 \\
(0)\end{array}$ & 35,8 & 0,9 \\
\hline 0 & $\begin{array}{r}200 \\
0\end{array}$ & 138,01 & 398,09 & $\begin{array}{l}\text { Kaltluftabfluß } \\
(0 \text { bis } 2000)\end{array}$ & $\begin{array}{r}1 \\
(4,4)\end{array}$ & $\begin{array}{r}2000 \\
(0)\end{array}$ & 175,6 & 488,3 \\
\hline 0 & 406 & 12,04 & 18,74 & $\begin{array}{l}\text { Leewirbel } \\
{[\mathrm{m}]}\end{array}$ & $\begin{array}{r}0 \\
(0)\end{array}$ & $\begin{array}{r}84 \\
(1,2)\end{array}$ & 12,9 & 18,4 \\
\hline
\end{tabular}

Anhang 9: Niederschlag im Harz nach DWD-Stationen ( $n=36)$ : Regressionskoeffizienten, Bestimmtheitsmaße und Werte des RMSE nach schrittweiser linearer Regression

\begin{tabular}{l:cc|rcccc}
\hline $\begin{array}{c}\text { Monat/ } \\
\text { Jahr }\end{array}$ & $\begin{array}{c}\mathrm{R}^{2} \\
{[\%]}\end{array}$ & $\begin{array}{c}\text { RMSE } \\
{[1 / 10 \mathrm{~mm}]}\end{array}$ & $\begin{array}{c}\text { Additive } \\
\text { Konst. }\end{array}$ & $\begin{array}{c}\text { Gelände- } \\
\text { höhe }\end{array}$ & Leewirbel & Luv & Rechtswert \\
\hline Jan & 81,0 & 146,20 & 254,30 & 1,510 & 0,703 & & \\
Feb & 85,6 & 86,93 & 214,47 & 1,083 & & 0,327 & \\
Mär & 88,9 & 89,44 & 263,83 & 1,272 & 0,521 & & \\
Apr & 87,0 & 61,85 & 385,02 & 0,824 & & 0,277 & \\
Mai & 64,2 & 73,90 & 16229,00 & 0,647 & & 0,148 & $-0,004362$ \\
Jun & 74,1 & 75,59 & 713,12 & 0,657 & & 0,312 & \\
Jul & 63,5 & 108,70 & 22746,00 & 0,905 & & 0,315 & $-0,006187$ \\
Aug & 69,3 & 82,39 & 564,22 & 0,651 & & 0,302 & \\
Sep & 76,0 & 86,57 & 343,87 & 0,798 & & 0,304 & \\
Okt & 86,5 & 77,33 & 281,87 & 0,984 & 0,394 & & \\
Nov & 87,5 & 107,10 & 280,96 & 1,426 & 0,613 & & \\
Dez & 81,2 & 167,71 & 321,73 & 1,750 & 0,762 & & \\
Jahr & 85,4 & 992,99 & 4606,49 & 12,331 & & 4,266 & \\
\hline
\end{tabular}


Anhang 10: Niederschlag im Harz nach HWW-Stationen $(n=65)$ : Regresionskoeffizienten, Bestimmtheitsmaße und Werte des RMSE nach schrittweiser linearer Regression

\begin{tabular}{l:cr|rcccc}
\hline $\begin{array}{c}\text { Monat/ } \\
\text { Jahr }\end{array}$ & $\begin{array}{c}\mathrm{R}^{2} \\
{[\%]}\end{array}$ & $\begin{array}{c}\text { RMSE } \\
{[1 / 10 \mathrm{~mm}]}\end{array}$ & $\begin{array}{c}\text { Additive } \\
\text { Konst. }\end{array}$ & $\begin{array}{c}\text { Gelände- } \\
\text { höhe }\end{array}$ & Leewirbel & Rechtswert & Hochwert \\
\hline Jan & 61,4 & 158,65 & 287,09 & 1,370 & 0,552 & & \\
Feb & 68,1 & 99,18 & 237,81 & 0,993 & 0,379 & & \\
Mär & 71,4 & 106,11 & 292,67 & 1,151 & 0,442 & & \\
Apr & 69,8 & 72,68 & 429,50 & 0,758 & 0,275 & & \\
Mai & 69,3 & 62,96 & 1296,04 & 0,661 & 0,151 & & \\
Jun & 59,1 & 88,69 & 664,03 & 0,729 & 0,239 & & \\
Jul & 64,3 & 97,88 & 36266,00 & 1,043 & 0,337 & $-0,009963$ & \\
Aug & 63,7 & 71,53 & 24607,00 & 0,751 & 0,251 & $-0,006695$ & \\
Sep & 65,3 & 89,47 & 332,35 & 0,840 & 0,264 & & \\
Okt & 66,6 & 96,71 & 276,02 & 0,936 & 0,326 & & \\
Nov & 69,5 & 134,46 & 239,89 & 1,392 & 0,537 & & \\
Dez & 65,1 & 186,92 & 292,90 & 1,749 & 0,672 & & \\
Jahr & 69,4 & 1149,98 & 4754,38 & 11,889 & 4,268 & & \\
\hline
\end{tabular}

Anhang 11: Harz, Ergebnisse für ein kombiniertes Datenkollektv (65 HWWund 36 DWD-Stationen); oben OLS-Regressionskoeffizienten, unten GLS-Regressionskoeffizienten und Semivariogramme

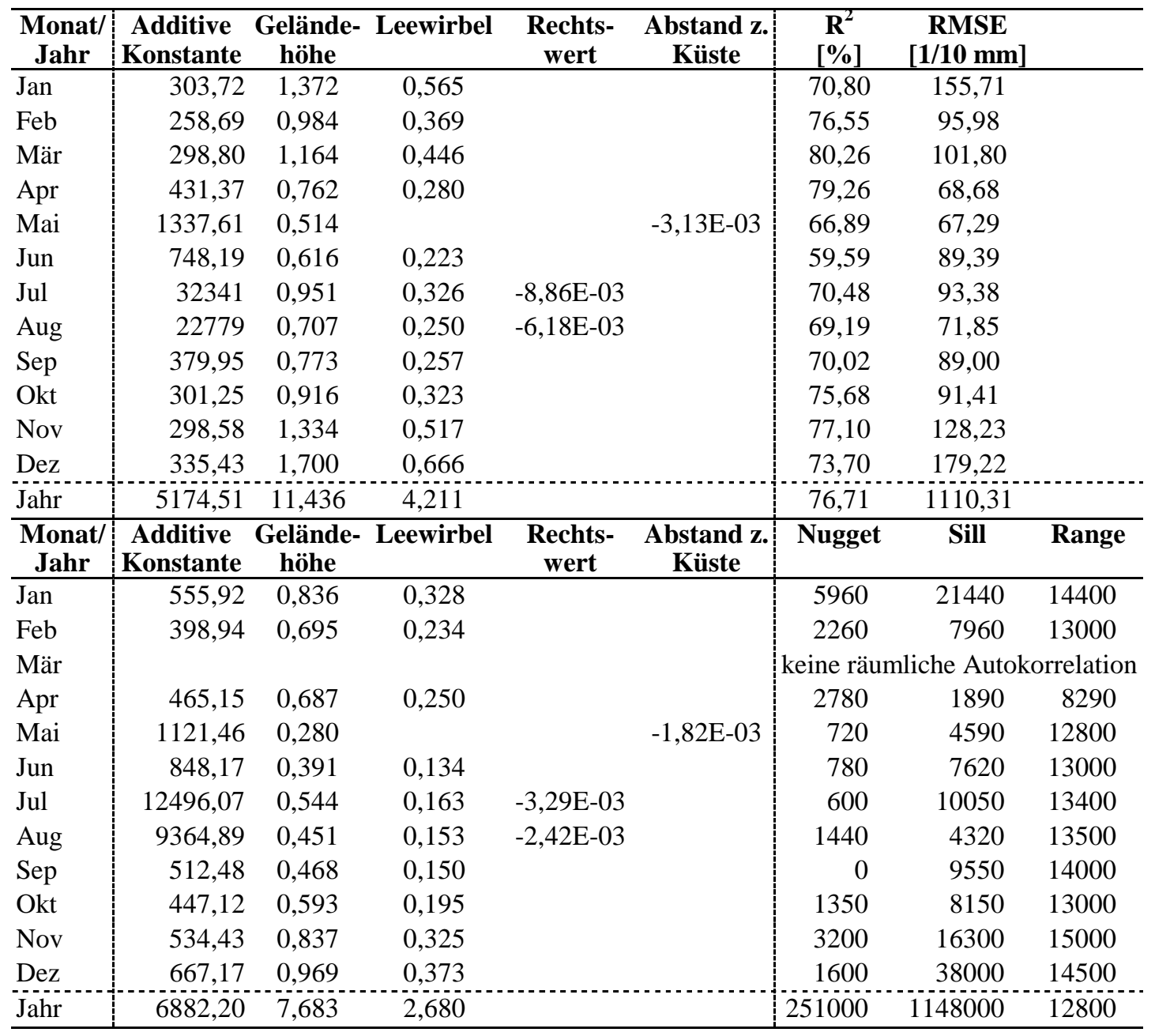


Anhang 12: Alternativmodelle zur Regionalisierung der Niederschlagssumme im Bergland (DWD/HWW-Datenkollektiv) ohne die Verwendung der räumlichen Variablen Lee-Effekt 50

\begin{tabular}{lrcccc}
\hline $\begin{array}{c}\text { Monat/ } \\
\text { Jahr }\end{array}$ & $\begin{array}{c}\mathrm{R}^{2} \\
{[\%]}\end{array}$ & $\begin{array}{c}\text { RMSE } \\
{[1 / 10 \mathrm{~mm}]}\end{array}$ & $\begin{array}{c}\text { Additive } \\
\text { Konstante }\end{array}$ & Geländehöhe & Leewirbel \\
\hline Mai & 81,2 & 63,48 & 555,91 & 0,562 & 0,150 \\
Jul & 80,1 & 94,33 & 551,47 & 0,783 & 0,296 \\
Aug & 81,1 & 73,81 & 577,28 & 0,630 & 0,246 \\
Sep & 83,0 & 79,23 & 400,17 & 0,729 & 0,256 \\
\hline
\end{tabular}

Anhang 13: Alternativmodelle zur Regionalisierung der relativen Luftfeuchte im Bergland ohne die Verwendung der räumlichen Variablen LeeEffekt 50

\begin{tabular}{lcccccc}
\hline $\begin{array}{c}\text { Monat/ } \\
\text { Jahr }\end{array}$ & $\begin{array}{c}\mathrm{R}^{2} \\
{[\%]}\end{array}$ & $\begin{array}{c}\text { RMSE } \\
{[\%]}\end{array}$ & $\begin{array}{c}\text { Additive } \\
\text { Konstante }\end{array}$ & $\begin{array}{c}\text { Gelände- } \\
\text { höhe }\end{array}$ & Bebauung & Wald \\
\hline Jan & 57,8 & 1,67 & 83,3 & $6,437 \mathrm{E}-03$ & & \\
Feb & 82,5 & 1,09 & 83,2 & $4,775 \mathrm{E}-03$ & $-6,50$ & \\
Sep & 66,4 & 1,77 & 78,6 & $8,227 \mathrm{E}-03$ & & \\
Okt & 52,5 & 1,45 & 81,6 & $5,028 \mathrm{E}-03$ & & \\
Nov & 61,5 & 1,77 & 83,0 & $7,374 \mathrm{E}-03$ & & \\
Dez & 54,2 & 1,65 & 83,6 & & & 6,54 \\
\hline
\end{tabular}




\section{Graphische Darstellung der Ergeb- nisse}

Die Regionalisierungmodelle zu den Klimaelementen und Depositionsdaten konnten im GIS ARC/Info graphisch dargestellt werden. Da die räumlichen Variablen nach dem in dieser Arbeit verfolgten Prinzip im GIS flächendeckend für das Zielgebiet Niedersachsen verfügbar sind, konnten die resultierenden Ergebnis-Raster durch einfache arithmetische Operationen hergeleitet werden. Im Falle der Niederschlagsergebnisraster wurde eine vorherige Interpolation nach dem gostatistischen Verfahren KRIGING mit dem Programm GSTAT 2.0 durchgeführt. Dieses Programmpaket wurde verwendet, da es eine gute Kompatibilität mit dem GIS ARC/Info ermöglicht.

Dargestellt werden hier die Modelle der Monate März, Juni, September und Dezember und der Mittelwert bzw. die Summe (Niederschlag) aller monatlichen Modelle. Diese vermittelt einen Eindruck über die Wirksamkeit aller verwendeten Regressoren und über eventuelle saisonale Effekte. Auf die Darstellung der Jahresmodelle wurde verzichtet, da sie in ihrer Zusammensetzung einzelnen Monatsmodellen ähneln.

Von den Depositionen werden die Regionalisierungsmodelle für die Einträge der fünf behandelten Stoffe und des pH-Werts im Freilandniederschlag dargestellt. Auf die graphische Darstellung der Modelle zur Regionalisierung der Bestandesanreicherungsfaktoren wurde wegen des geringen Datenumfangs verzichtet.

Als Beispiel für eine abgeleitete Informationslage wird die Vegetationszeit dargestellt (Zahl der Tage mit einer mittleren Lufttemperatur über $10^{\circ} \mathrm{C}$ ). Weitere Beispiele für mögliche Anwendungen der Regionalisierungsmodelle finden sich bei JANSEN et al. (in Vorb.). 
Ergebnisdarstellung 1: Umsetzung des Regionalisierungsmodells für die durchschnittliche Lufttemperatur im März in den Jahren von 1961 bis 1990

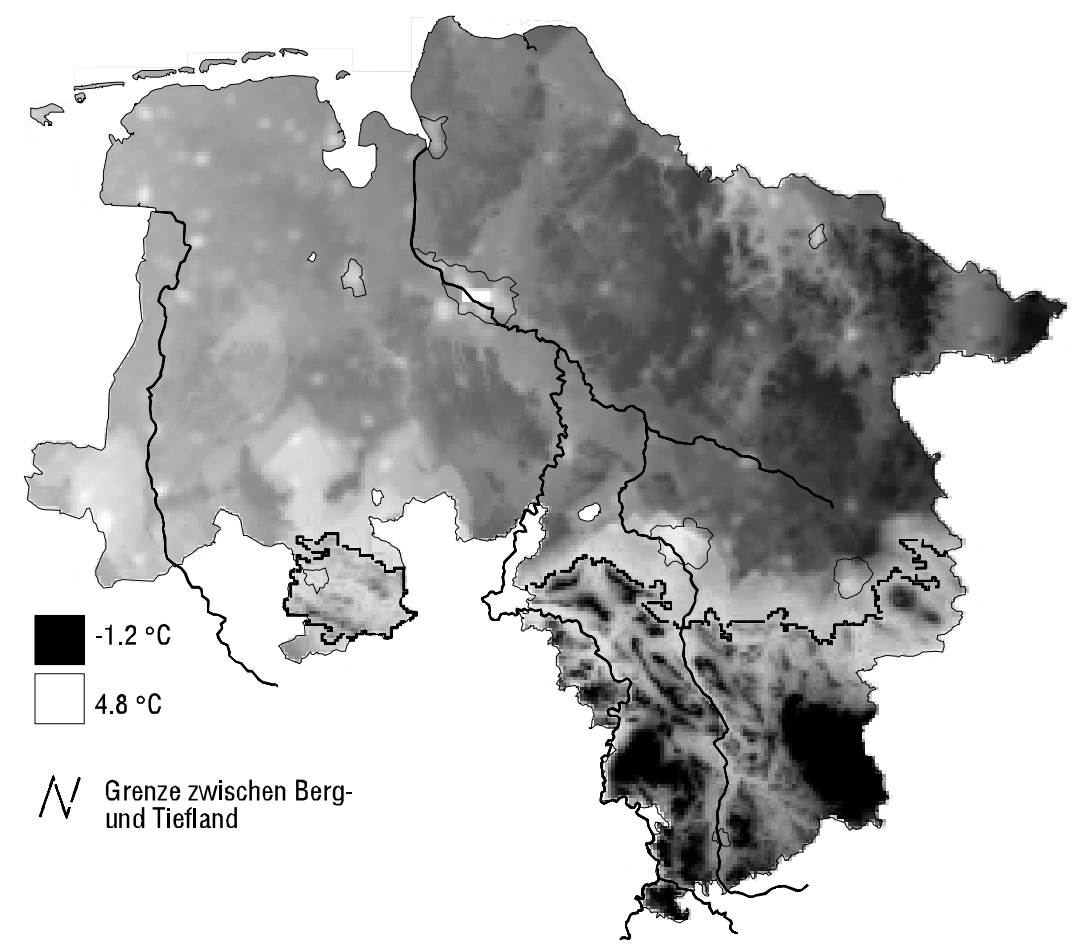

Ergebnisdarstellung 2: Umsetzung des Regionalisierungsmodells für die durchschnittliche Lufttemperatur im Juni in den Jahren von 1961 bis 1990

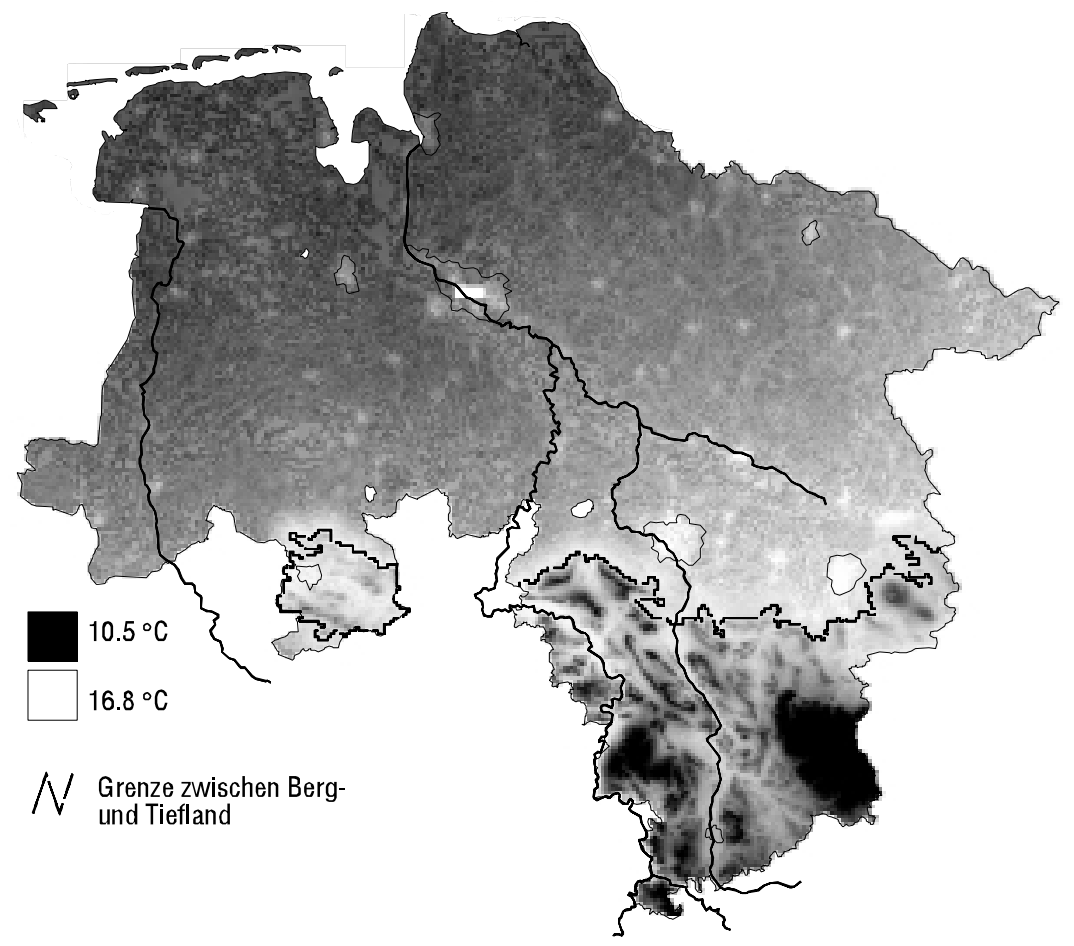


Ergebnisdarstellung 3: Umsetzung des Regionalisierungsmodells für die durchschnittliche Lufttemperatur im September in den Jahren von 1961 bis 1990

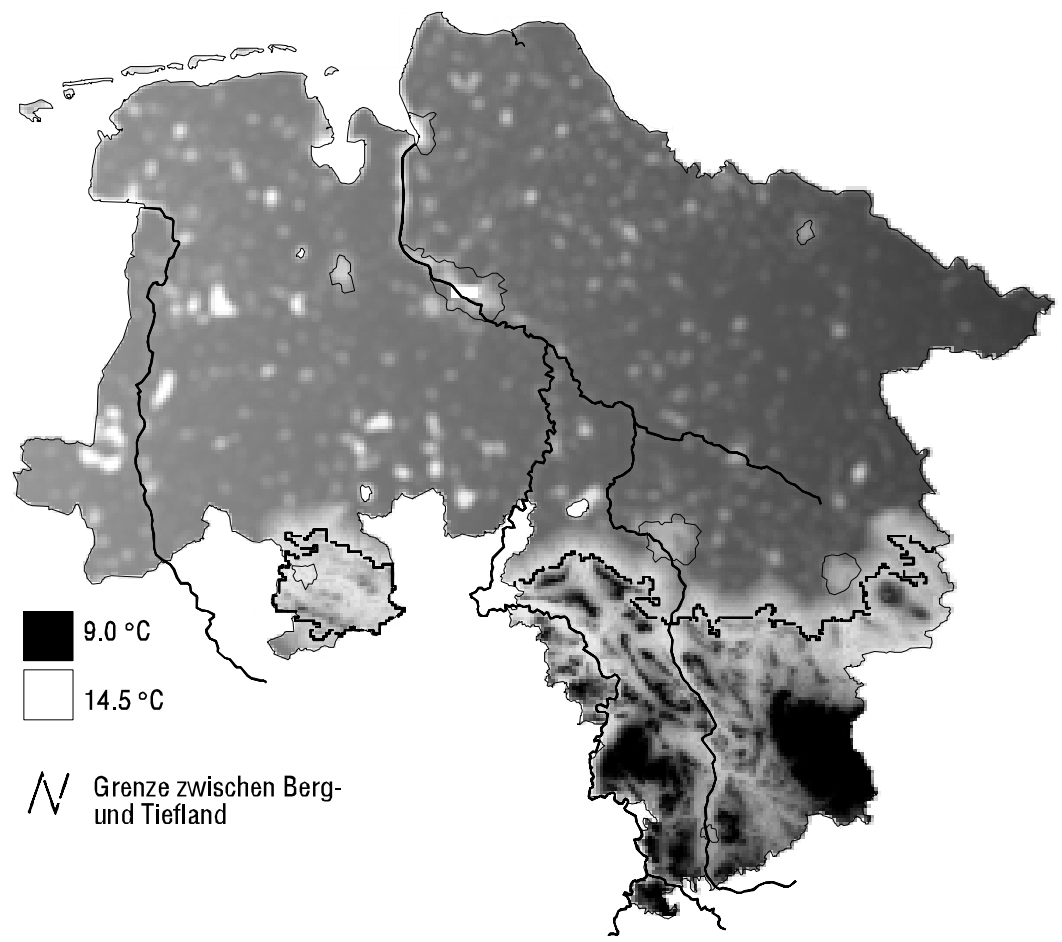

Ergebnisdarstellung 4: Umsetzung des Regionalisierungsmodells für die durchschnittliche Lufttemperatur im Dezember in den Jahren von 1961 bis 1990

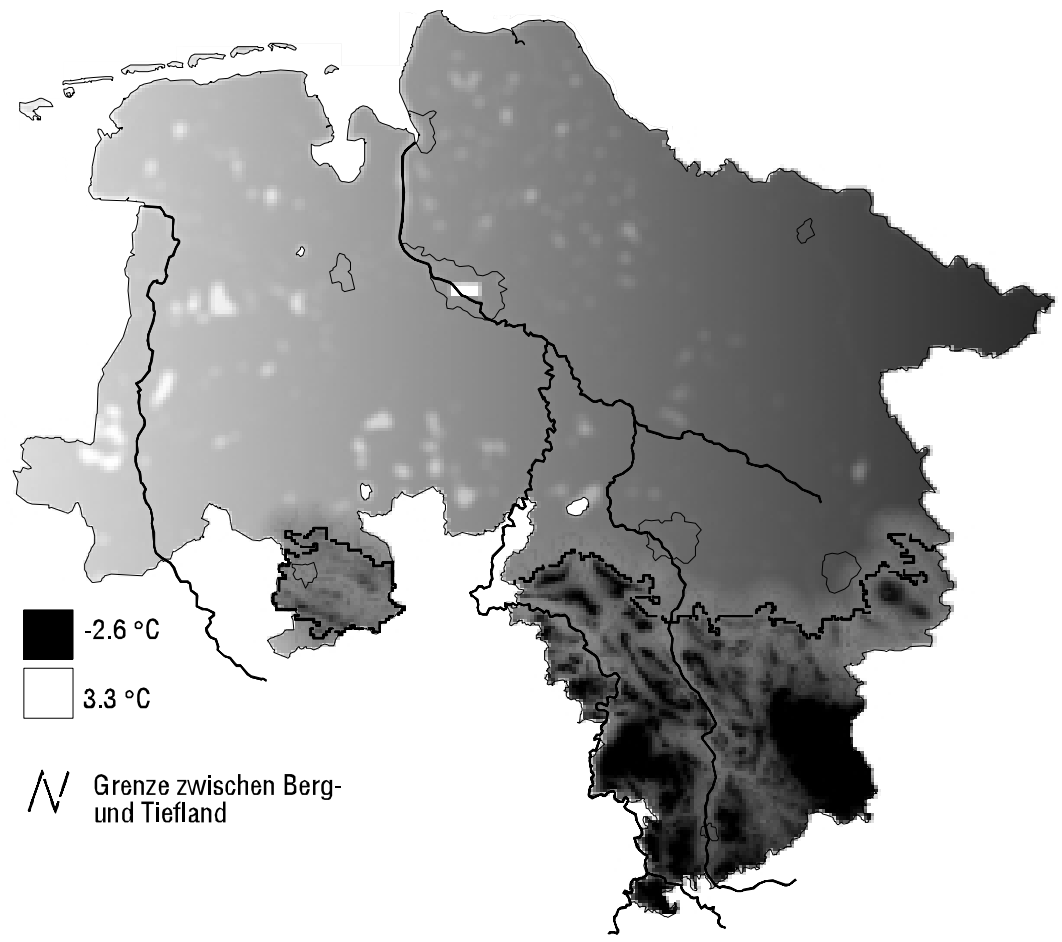


Ergebnisdarstellung 5: Durchschnittliche jährliche Lufttemperatur in den Jahren von 1961 bis 1990 (Mittelwert der monatlichen Regionalisierungsmodelle)

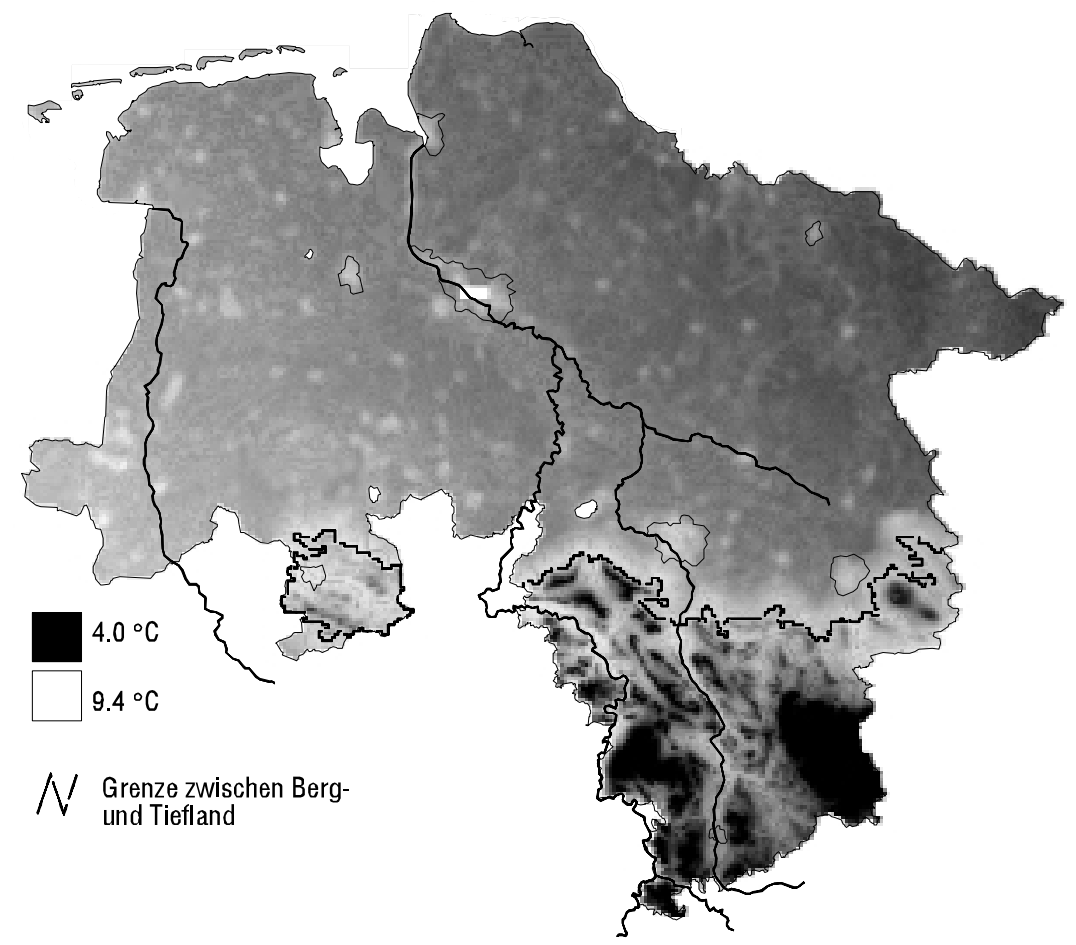

Ergebnisdarstellung 6: Umsetzung des Regionalisierungsmodells für die durchschnittliche Luftfeuchte im März in den Jahren von 1961 bis 1990

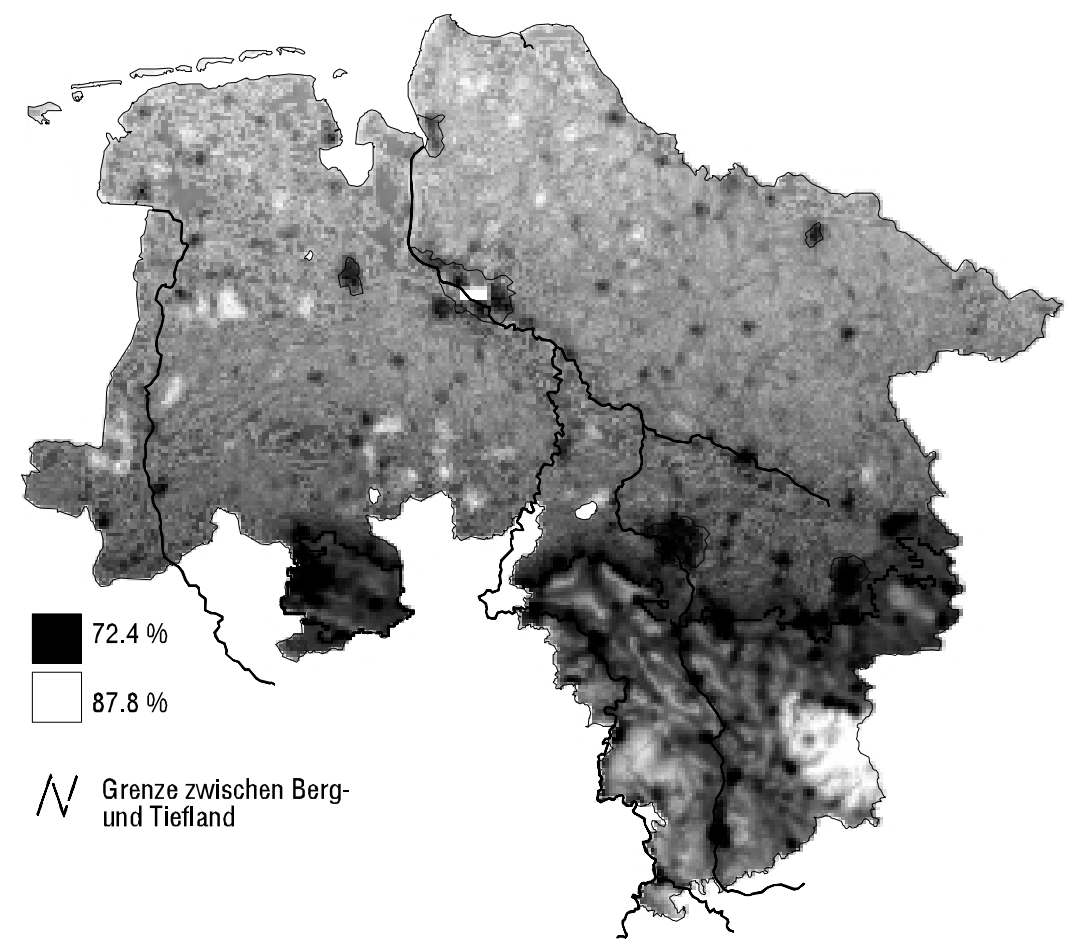


Ergebnisdarstellung 7: Umsetzung des Regionalisierungsmodells für die durchschnittliche Luftfeuchte im Juni in den Jahren von 1961 bis 1990

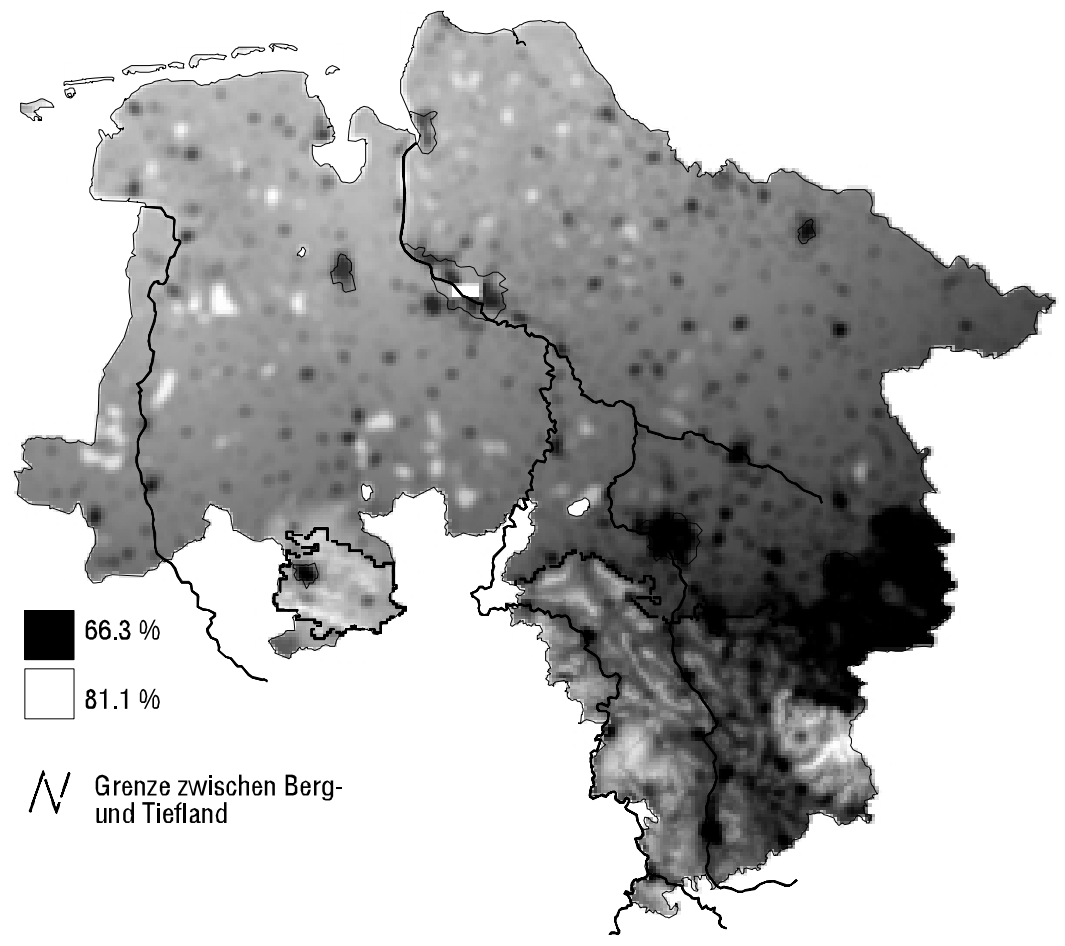

Ergebnisdarstellung 8: Umsetzung des Regionalisierungsmodells für die durchschnittliche Luftfeuchte im September in den Jahren von 1961 bis 1990

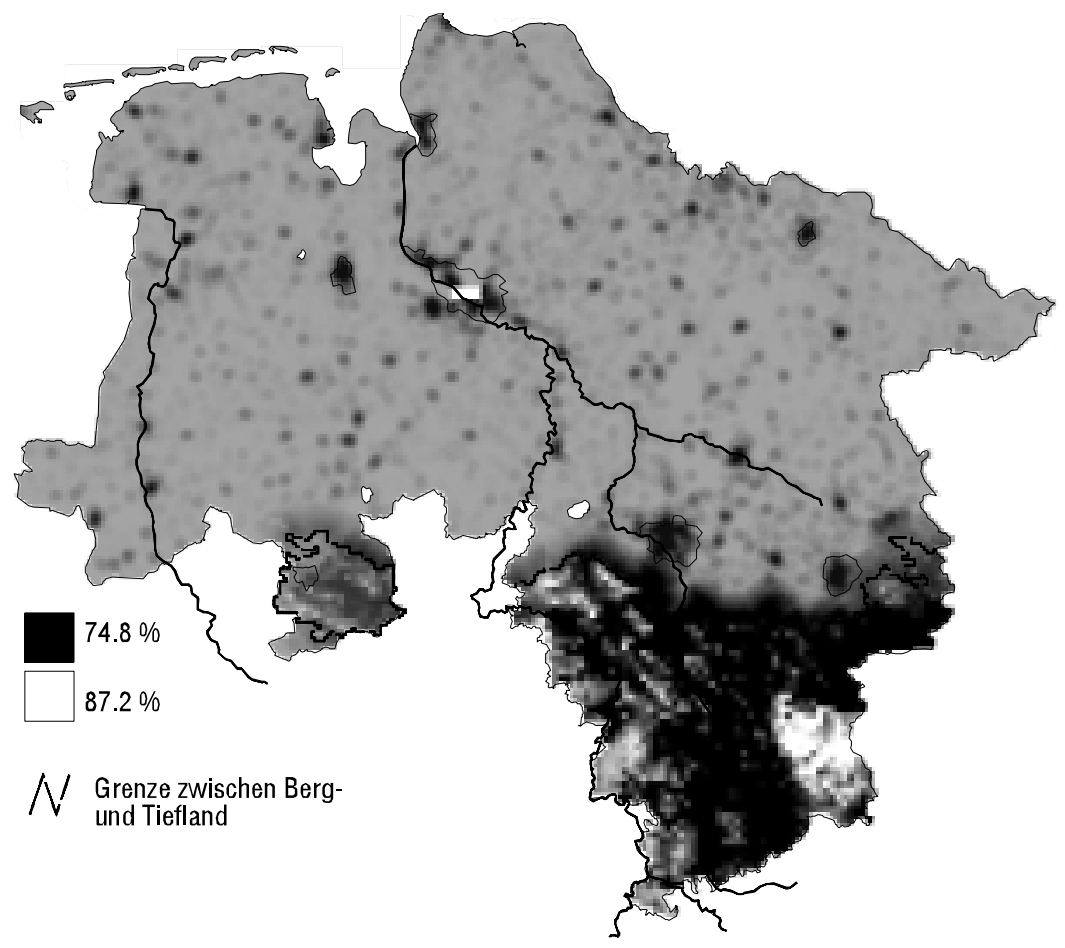


Ergebnisdarstellung 9: Umsetzung des Regionalisierungsmodells für die durchschnittliche Luftfeuchte im Dezember in den Jahren von 1961 bis 1990

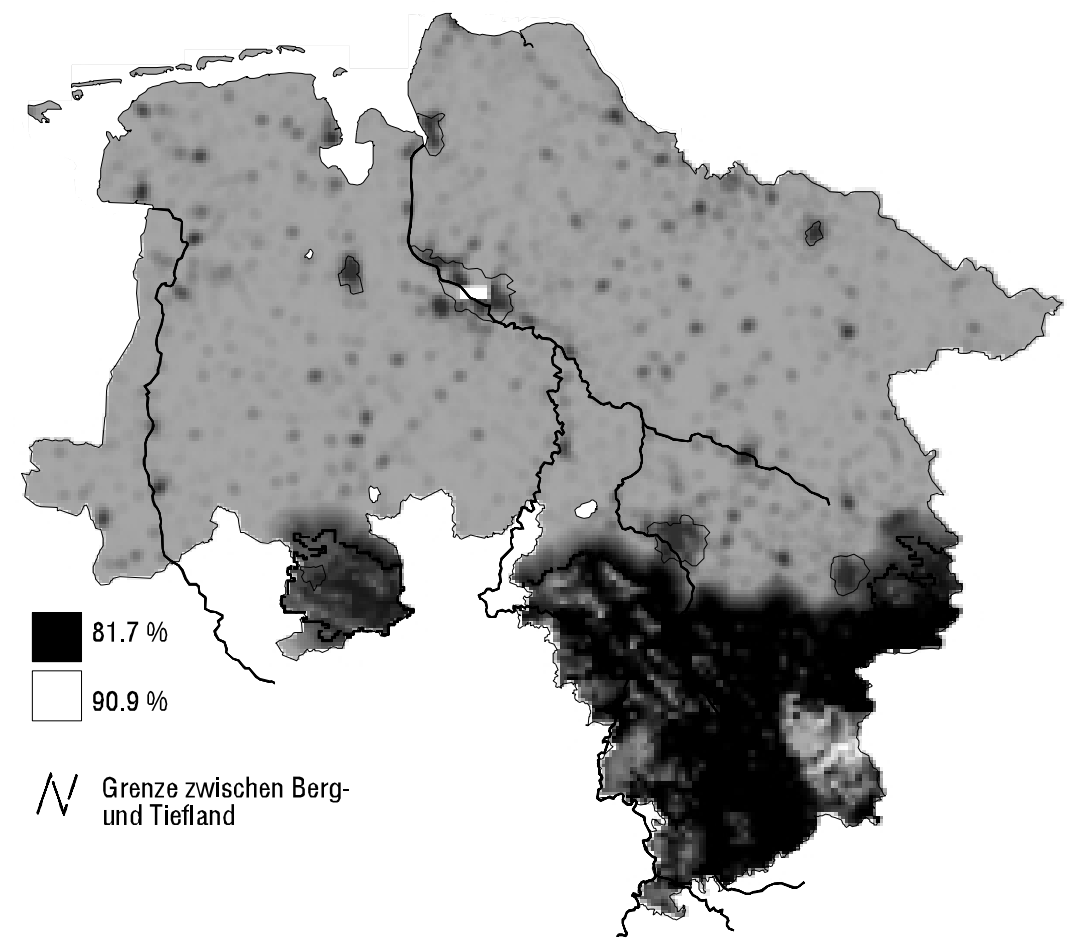

Ergebnisdarstellung 10: Durchschnittliche jährliche Luftfeuchte in den Jahren von 1961 bis 1990 (Mittelwert der monatlichen Regionalisierungsmodelle)

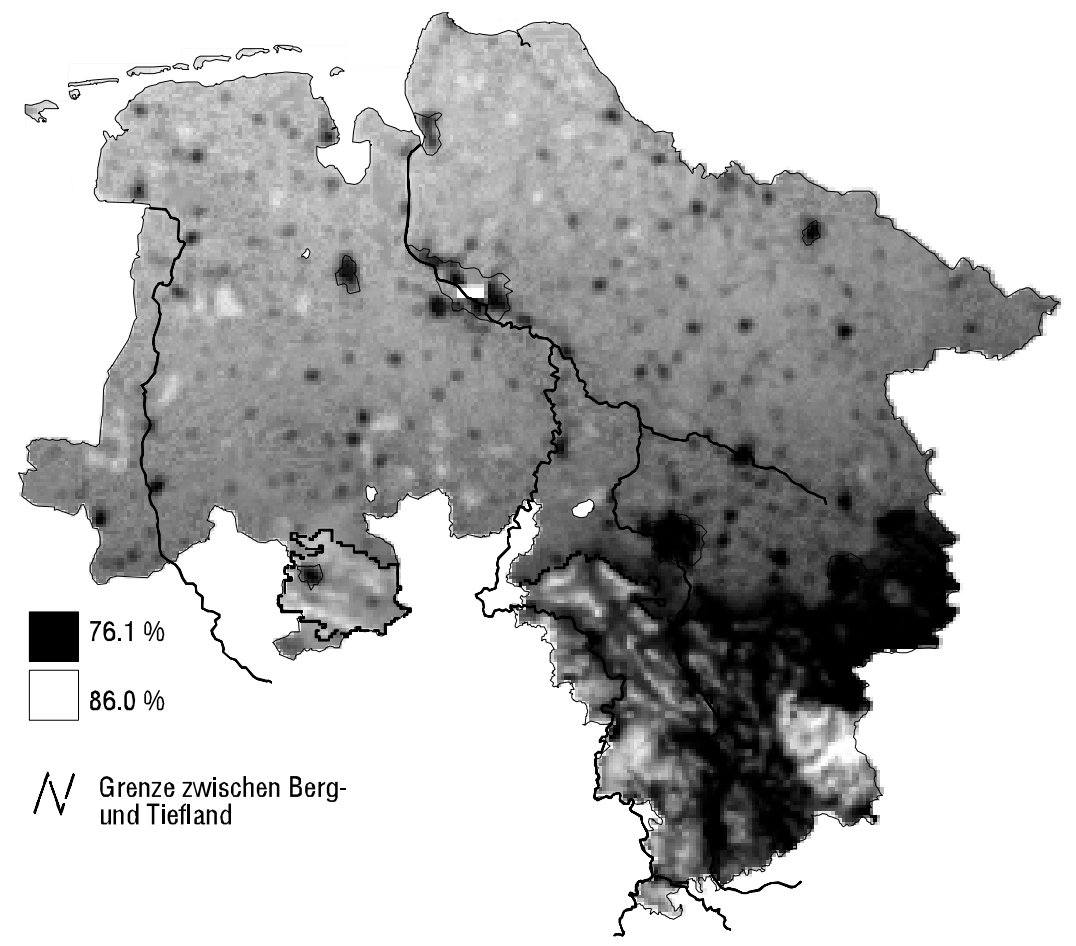


Ergebnisdarstellung 11: Umsetzung des Regionalisierungsmodells für die durchschnittliche Niederschlagssumme im März in den Jahren von 1961 bis 1990

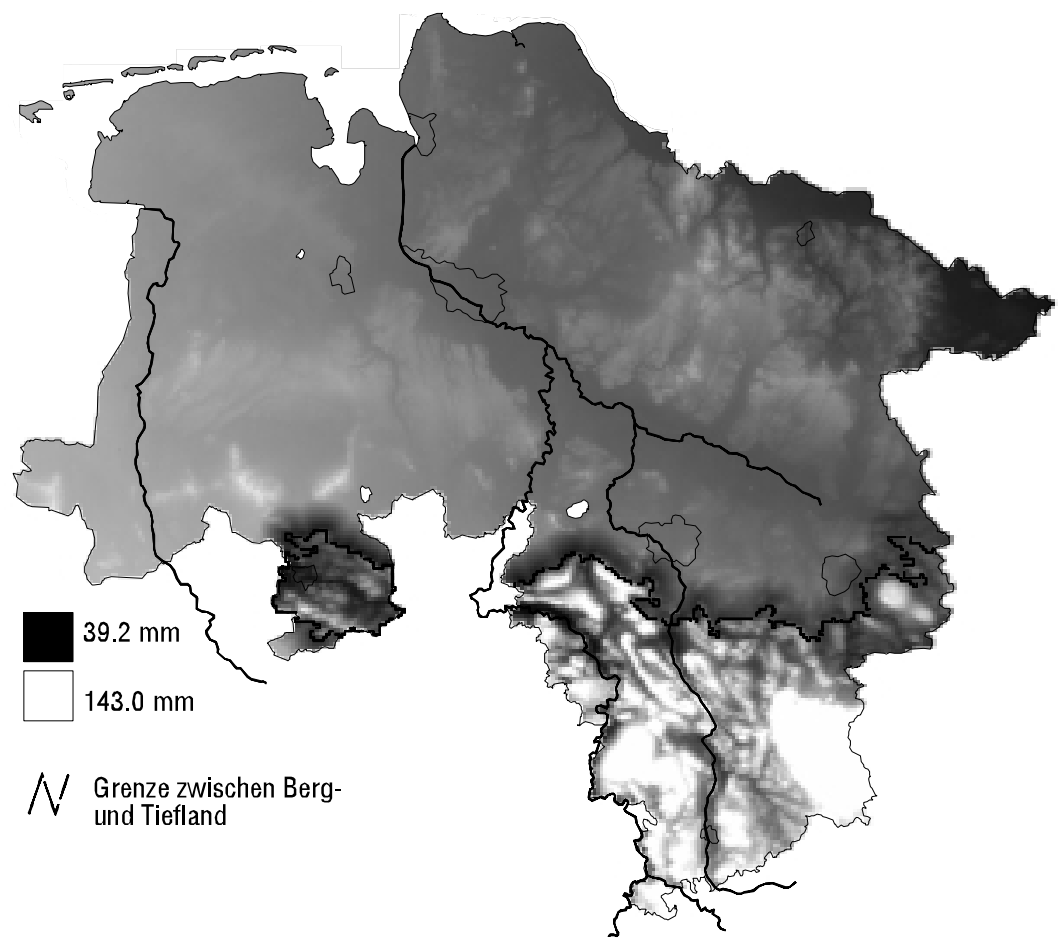

Ergebnisdarstellung 12: Umsetzung des Regionalisierungsmodells für die durchschnittliche Niederschlagssumme im Juni in den Jahren von 1961 bis 1990

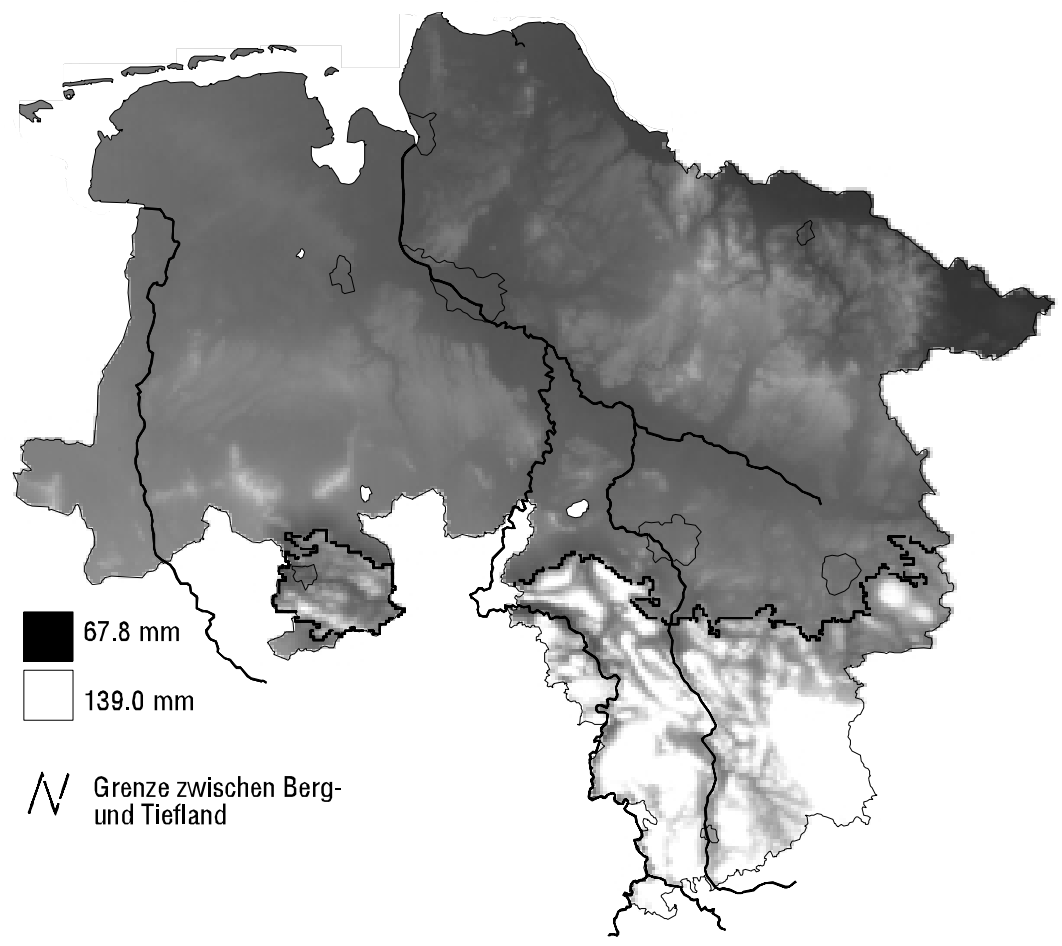


Ergebnisdarstellung 13: Umsetzung des Regionalisierungsmodells für die durchschnittliche Niederschlagssumme im September in den Jahren von 1961 bis 1990

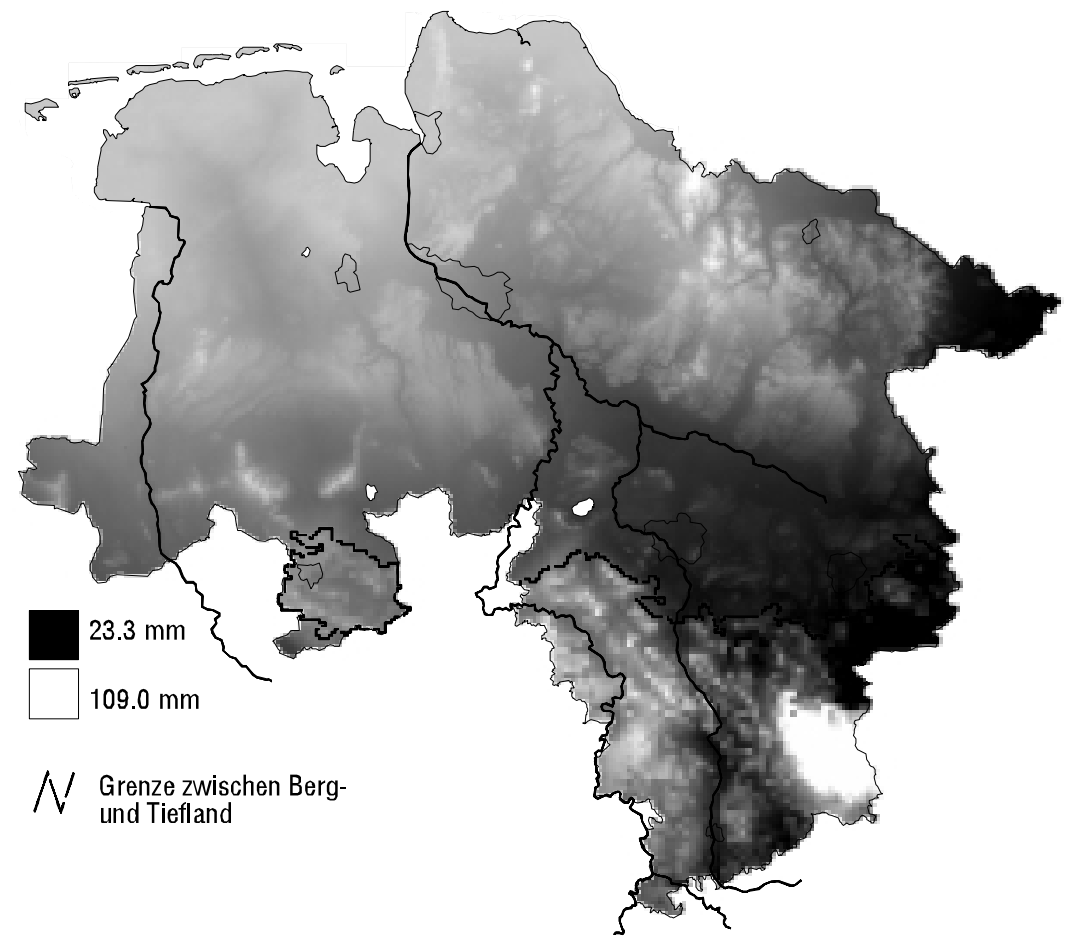

Ergebnisdarstellung 14: Umsetzung des Regionalisierungsmodells für die durchschnittliche Niederschlagssumme im Dezember in den Jahren von 1961 bis 1990

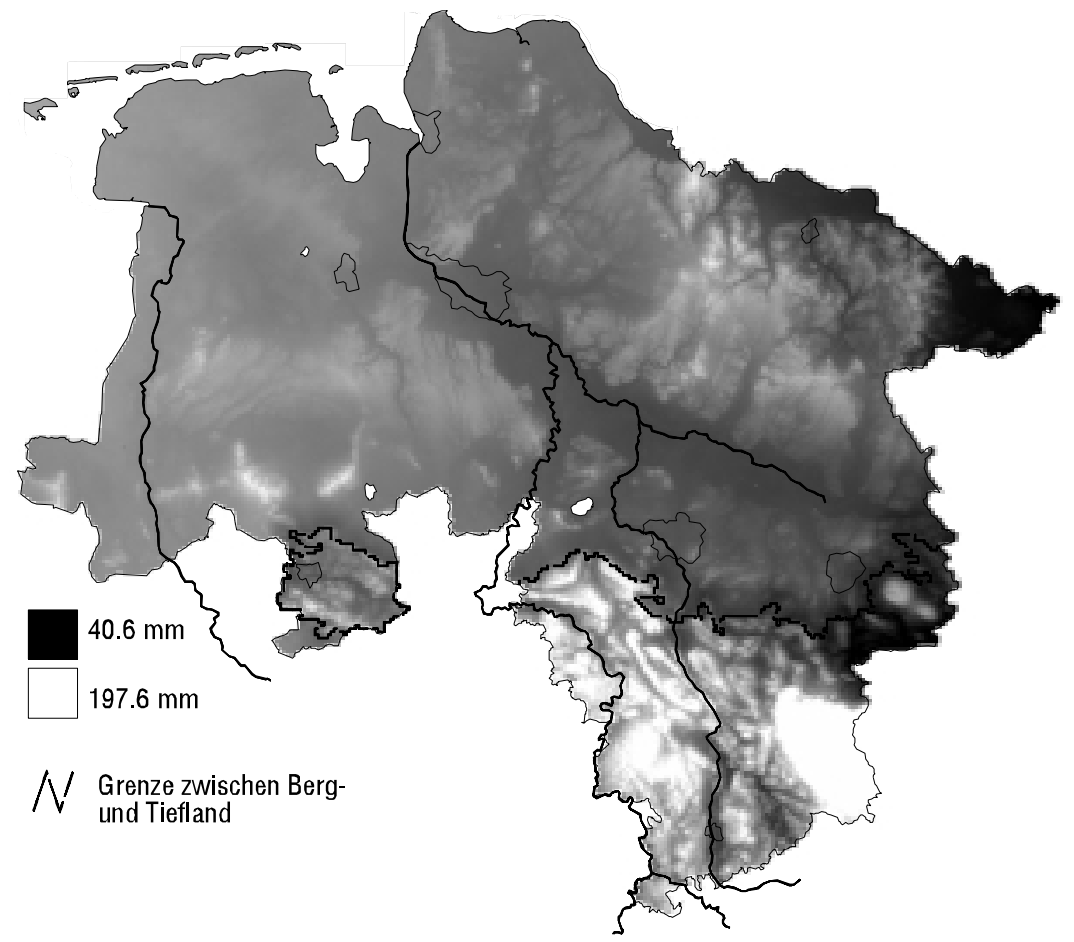


Ergebnisdarstellung 15: Durchschnittliche jährliche Niederschlagssumme in den Jahren von 1961 bis 1990 (Summe der monatlichen Regionalisierungsmodelle)

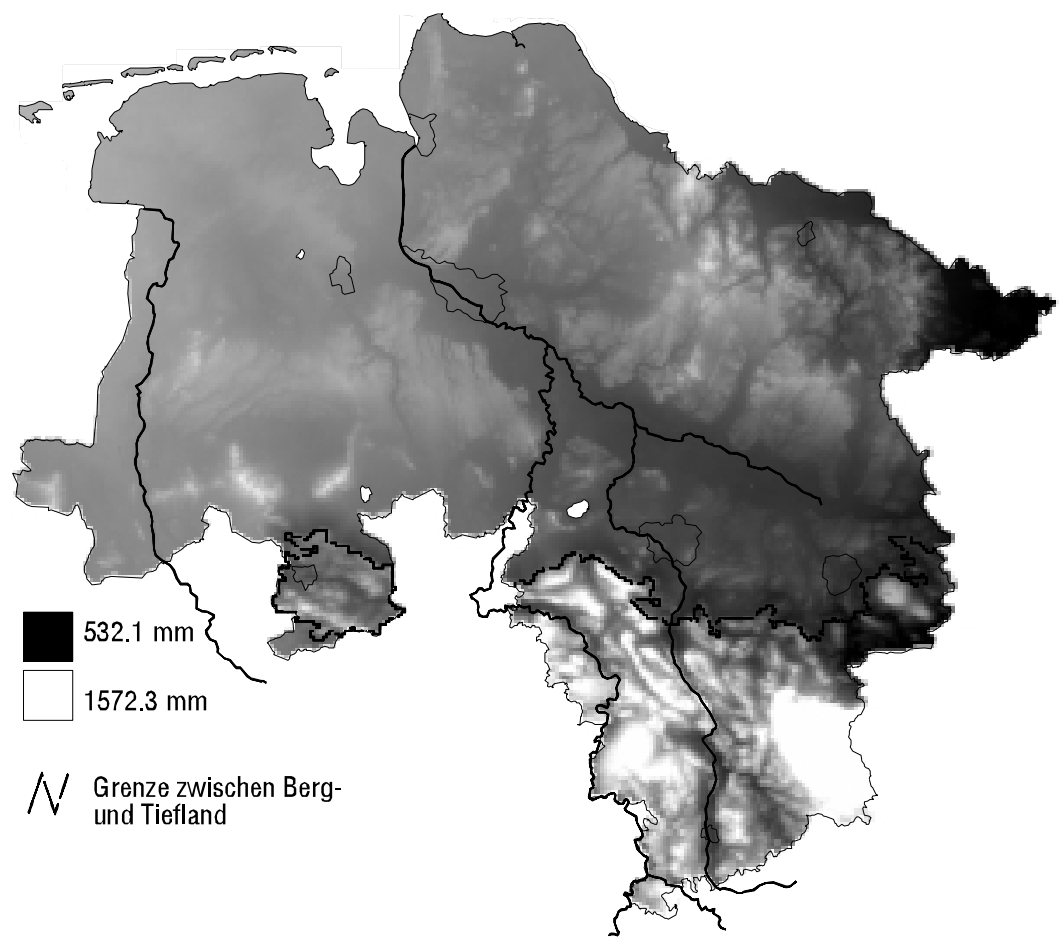

Ergebnisdarstellung 16: Umsetzung des Regionalisierungsmodells für den durchschnittlichen jährlichen Sulfat-Schwefeleintrag im Freilandniederschlag in den Jahren 1986 bis 1990

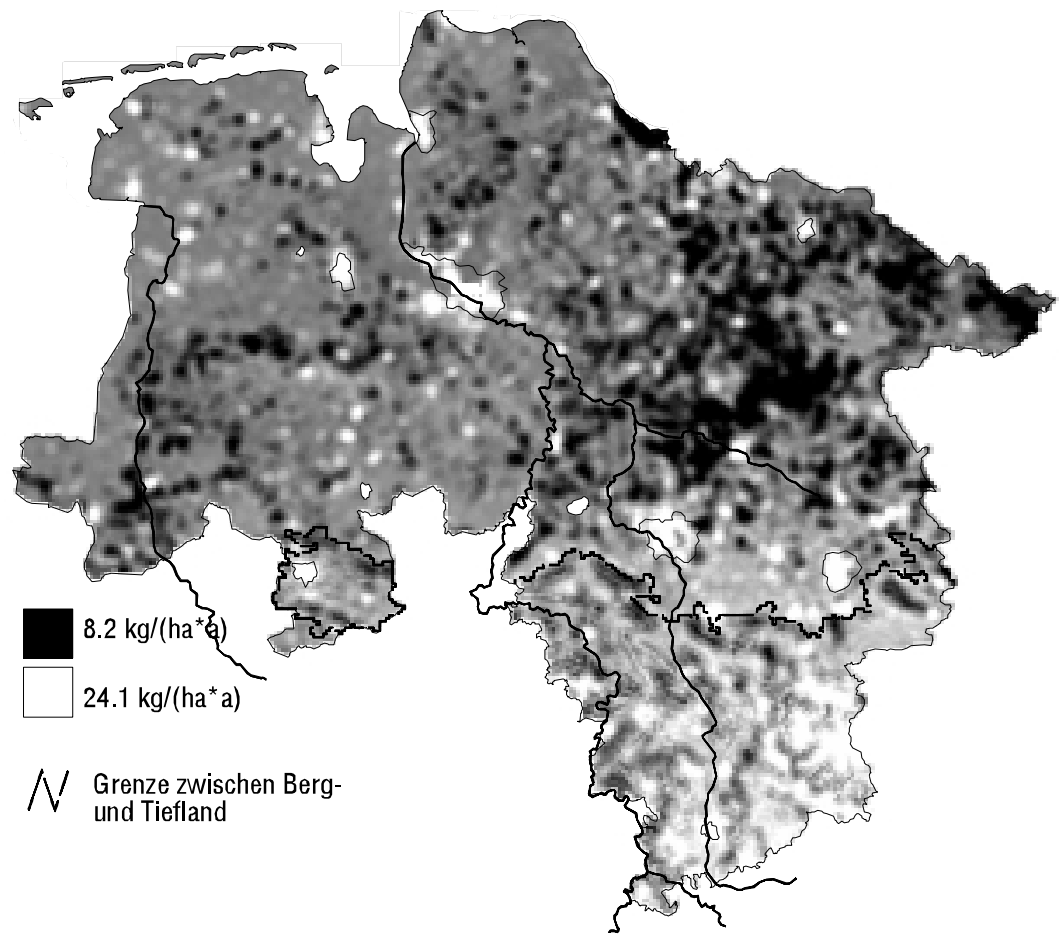


Ergebnisdarstellung 17: Umsetzung des Regionalisierungsmodells für den durchschnittlichen jährlichen Nitrat-Stickstoffeintrag im Freilandniederschlag in den Jahren 1986 bis 1990

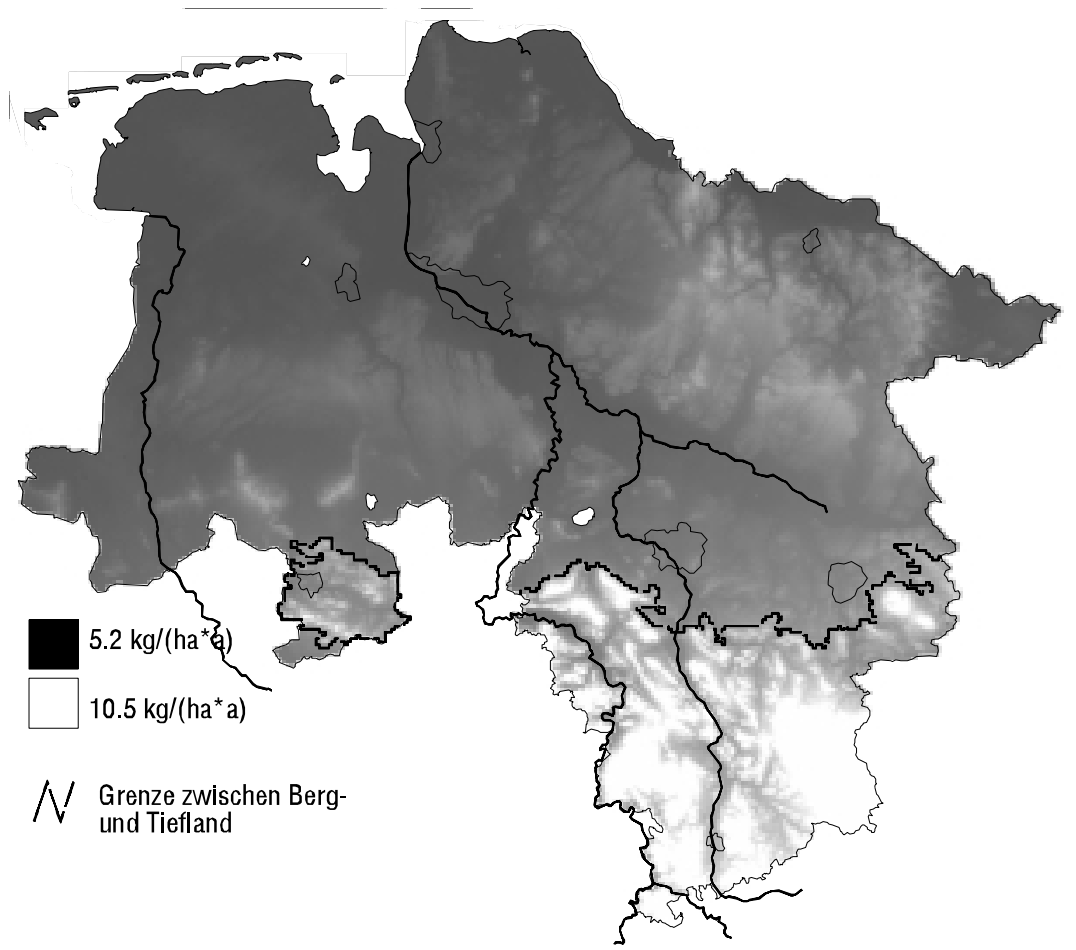

Ergebnisdarstellung 18: Umsetzung des Regionalisierungsmodells für den durchschnittlichen jährlichen Ammonium-Stickstoffeintrag im Freilandniederschlag in den Jahren 1986 bis 1990

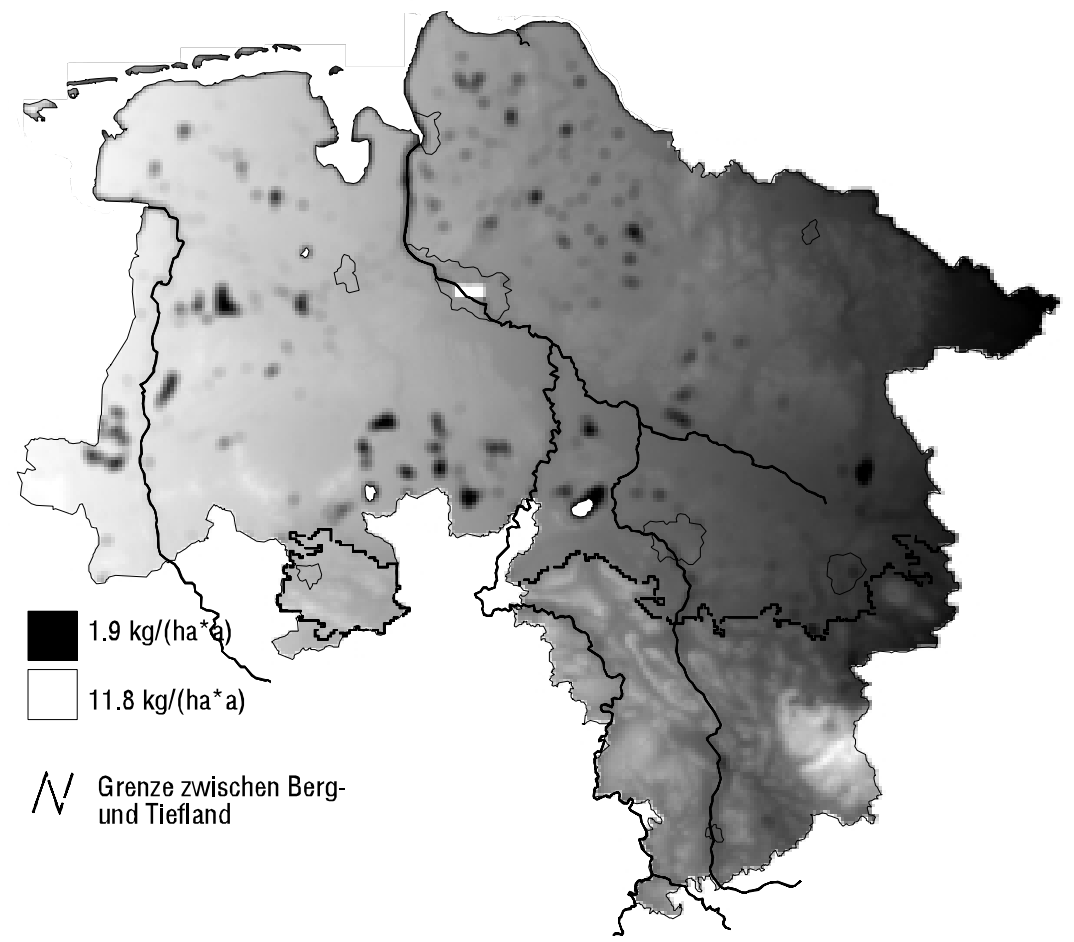


Ergebnisdarstellung 19: Umsetzung des Regionalisierungsmodells für den durchschnittlichen jährlichen Chloreintrag im Freilandniederschlag in den Jahren 1986 bis 1990

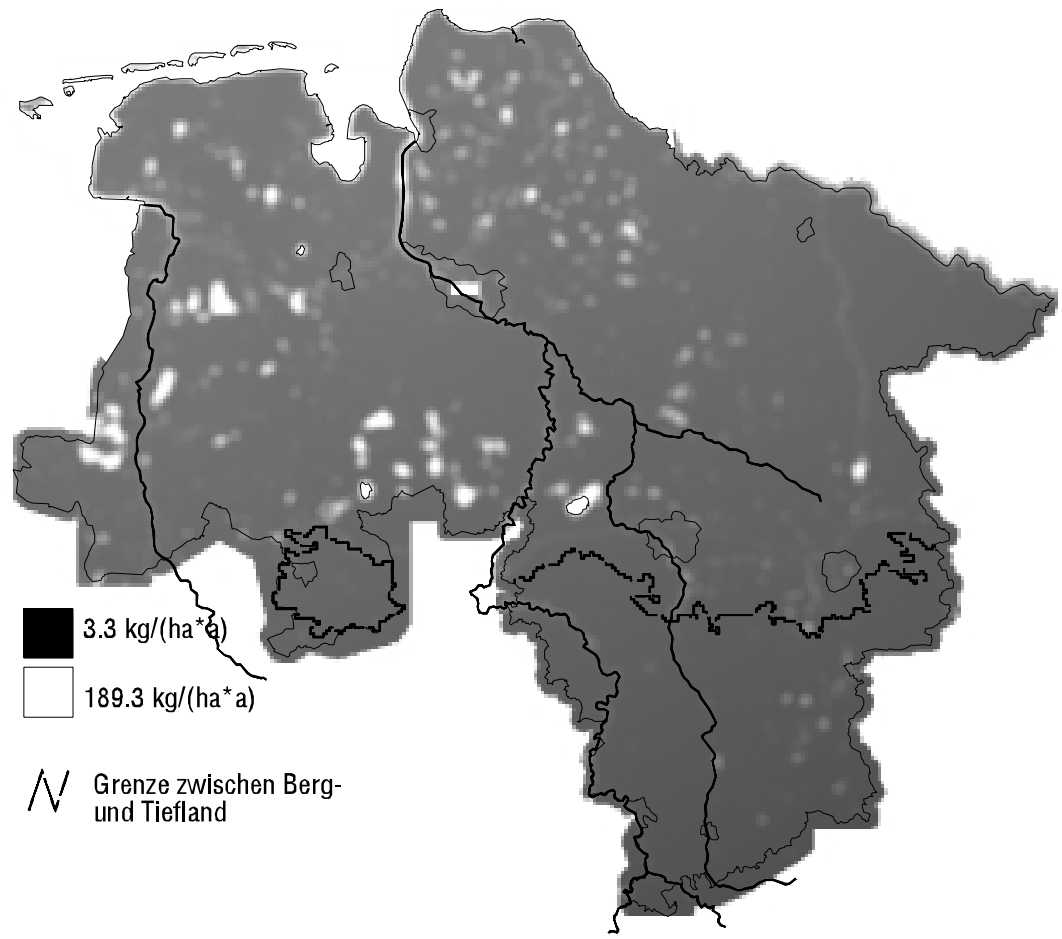

Ergebnisdarstellung 20: Umsetzung des Regionalisierungsmodells für den durchschnittlichen jährlichen Protoneneintrag im Freilandniederschlag in den Jahren 1986 bis 1990

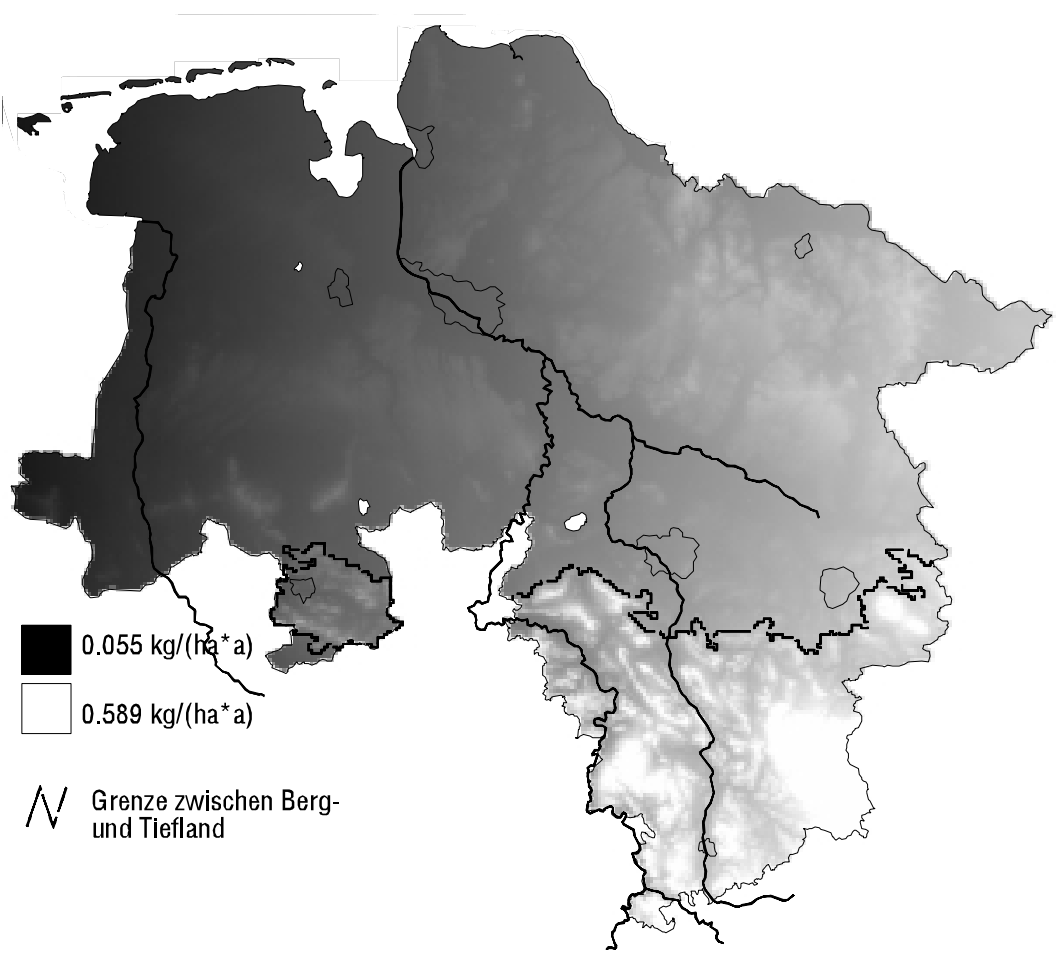


Ergebnisdarstellung 21: Umsetzung des Regionalisierungsmodells für den durchschnittlichen pH-Wert im Freilandniederschlag in den Jahren 1986 bis 1990

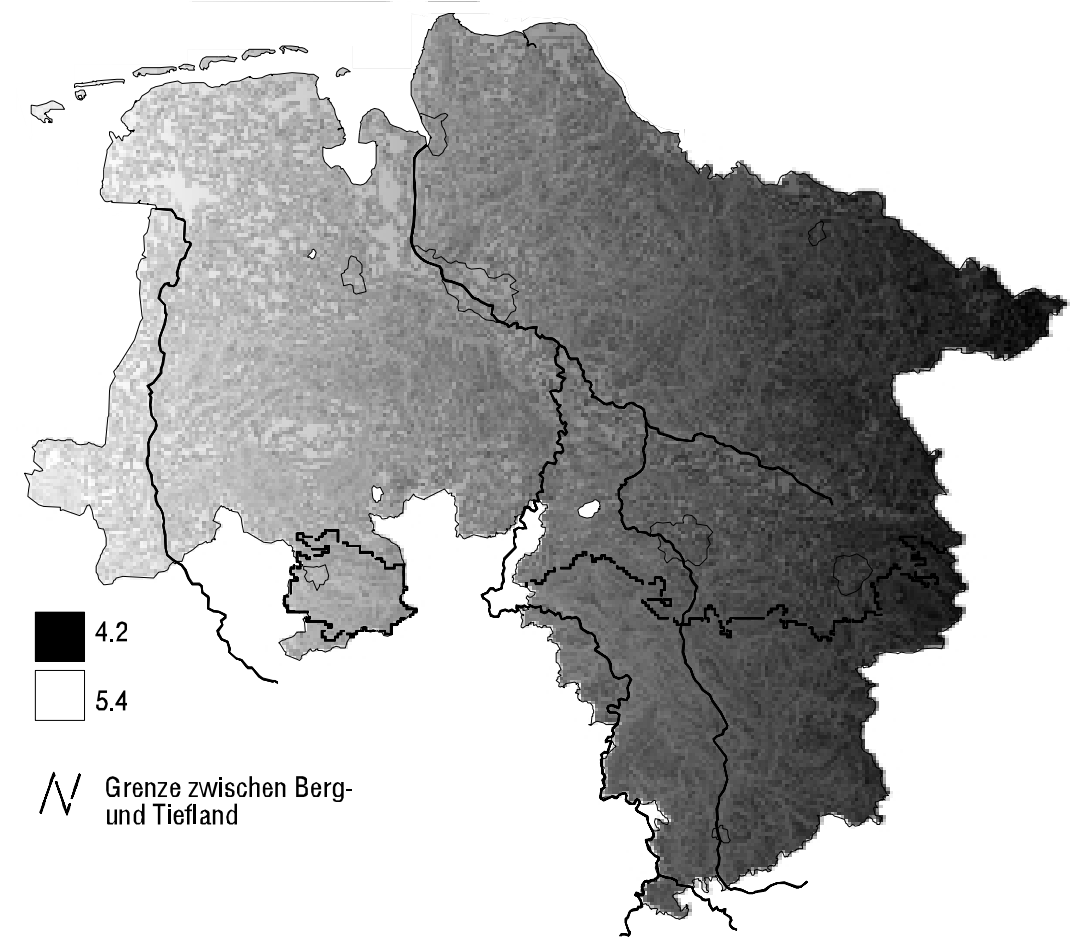

Ergebnisdarstellung 22: Vegetationszeit (Tage über $10^{\circ} \mathrm{C}$ ), abgeleitet aus den monatlichen Regressionsmodellen für die Lufttemperatur

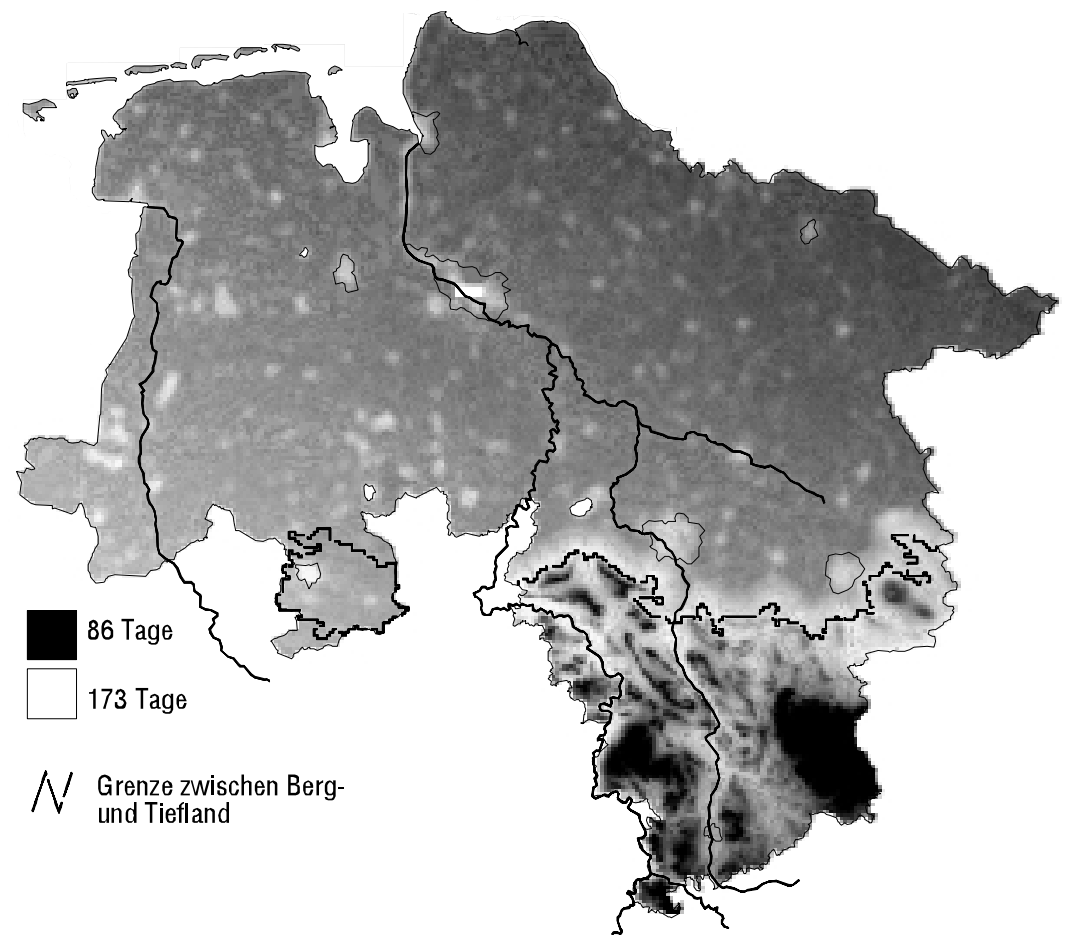




\section{Lebenslauf Volker Mues}

\section{Bäderstr. 31, 24214 NOER}

\section{Angaben zur Person}

17.03.1969 geboren in Frankfurt a. M. als zweites Kind des Dipl.- Bauingenieurs Leopold Mues und seiner Ehefrau Magdalene, geb. Bühre; eine Schwester:

Petra Mues (geb. 1968)

Aufgewachsen in Karben, Wetteraukreis / Hessen, Ortsteil Burg- Gräfenrode.

31.05.1996 Heirat mit Sabine Mues, geb. Rhades, Dipl. Ing. agrar.

26.01.1997 Geburt des ersten Sohnes, Jan Mues

7.12.1998 Geburt der ersten Tochter, Lena Mues

\section{$\underline{\text { Schulische Ausbildung }}$}

1975-1979 Grundschule Karben

1979-1988 Augustiner- Gymnasium Friedberg / Hessen

Abschluß: Abitur

$\underline{\text { Wehrdienst }}$

Jul. 1988 -

Sep. 1989 Bundeswehr W15; (Unteroffizier d. Res.)

\section{Berufliche Ausbildung und Tätigkeiten}

WS 89/90-

WS 94/95 Studium der Forstwissenschaft an der Georg-August-Universität Göttingen

WS 89/90 Praktikum im hessischen staatlichen Forstamt Bad Homburg

WS 91/92 Vordiplom

Feb. 1994-

Apr. 1994 Praktikum in Quintanar de la Sierra / Spanien

Jan. 1995 Diplom

Feb. 1995-

Apr. 1995 Tätigkeit bei der Maschinenhandelsgesellschaft mbH Müller Minden

Juli 1995-

Apr. 2000 Promotion an der Fakultät für Forstwissenschaft und Waldökologie der Universität Göttingen; im Rahmen des Forschungszentrums Waldökosysteme Tätigkeiten als wissenschaftliche Hilfskraft vorwiegend im Bereich der Anwendung geographischer Informationssysteme (GIS) und der Lehre

Seit 1.1.2000 Selbständig im Bereich EDV-Beratung GIS 
Volker Mues

25. Jan 2000

Bäderstr. 31

D-24214 Noer

\section{Eidesstattliche Versicherung}

Hiermit versichere ich gemäß $§ 4$ der Promotionsordnung der Fakultät für Forstwissenschaften und Waldökologie der Georg-August-Universität Göttingen in der Fassung vom 8. Januar 1986, daß ich die vorliegende Dissertation selbständig und ohne unerlaubte Hilfe angefertigt habe. 